Supporting Information

\title{
Synthesis of Trialkylamines with Extreme Steric Hindrance and Their Decay by a Hofmann-like Elimination Reaction
}

† Klaus Banert, ${ }^{*},{ }^{\dagger}$ Manfred Hagedorn,${ }^{\dagger}$ Manuel Heck, ${ }^{\dagger}$ Raphael Hertel, ${ }^{\dagger}$ Andreas Ihle, ${ }^{\dagger}$ Ioana Müller, ${ }^{\dagger}$ Tom Pester, ${ }^{\dagger}$ Tharallah Shoker ${ }^{\dagger}$ and Paul R. Rablen ${ }^{*, \ddagger}$

†Organic Chemistry, Chemnitz University of Technology, Strasse der Nationen 62, 09111 Chemnitz, Germany

‡ Department of Chemistry and Biochemistry, Swarthmore College, 500 College Ave., Swarthmore, Pennsylvania 19081, United States.

† Prof. Dr. Klaus Banert, passed away on September 19, 2020. 


\section{Table of Contents}

NMR spectra.

S-3

Kinetic data

S-82

Computational details. 


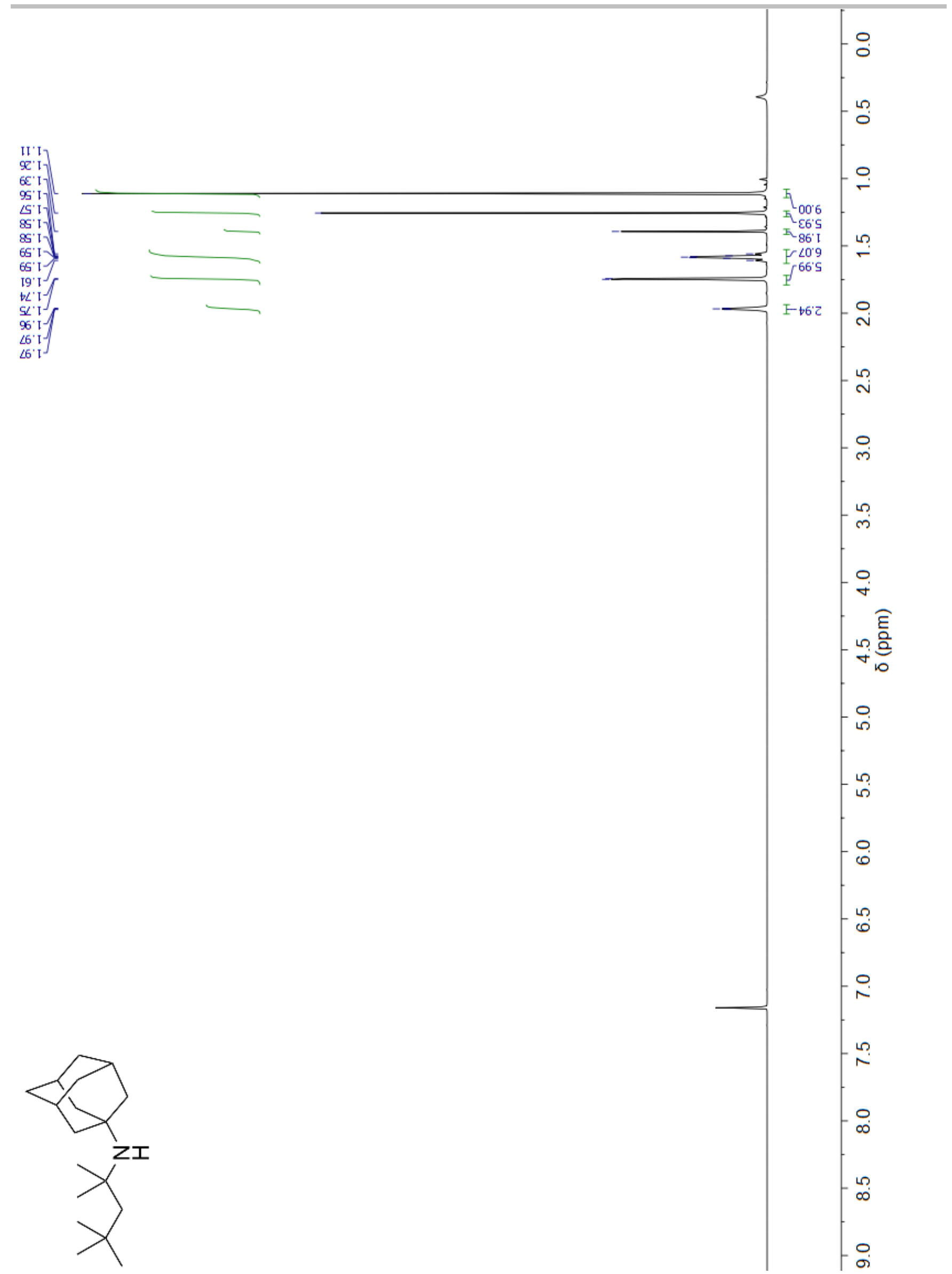

Figure S-1: ${ }^{1} \mathrm{H}$ NMR spectrum of $\mathrm{N}$-(1-adamantyl)-tert-octylamine in $\mathrm{C}_{6} \mathrm{D}_{6}$. 


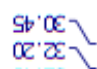

It $\alpha \frac{1}{5}$

क. $\alpha$

$90^{\prime} \angle \mathcal{E}-$

$9<96-$

tE'

tE' $9 S-$

๙6 6
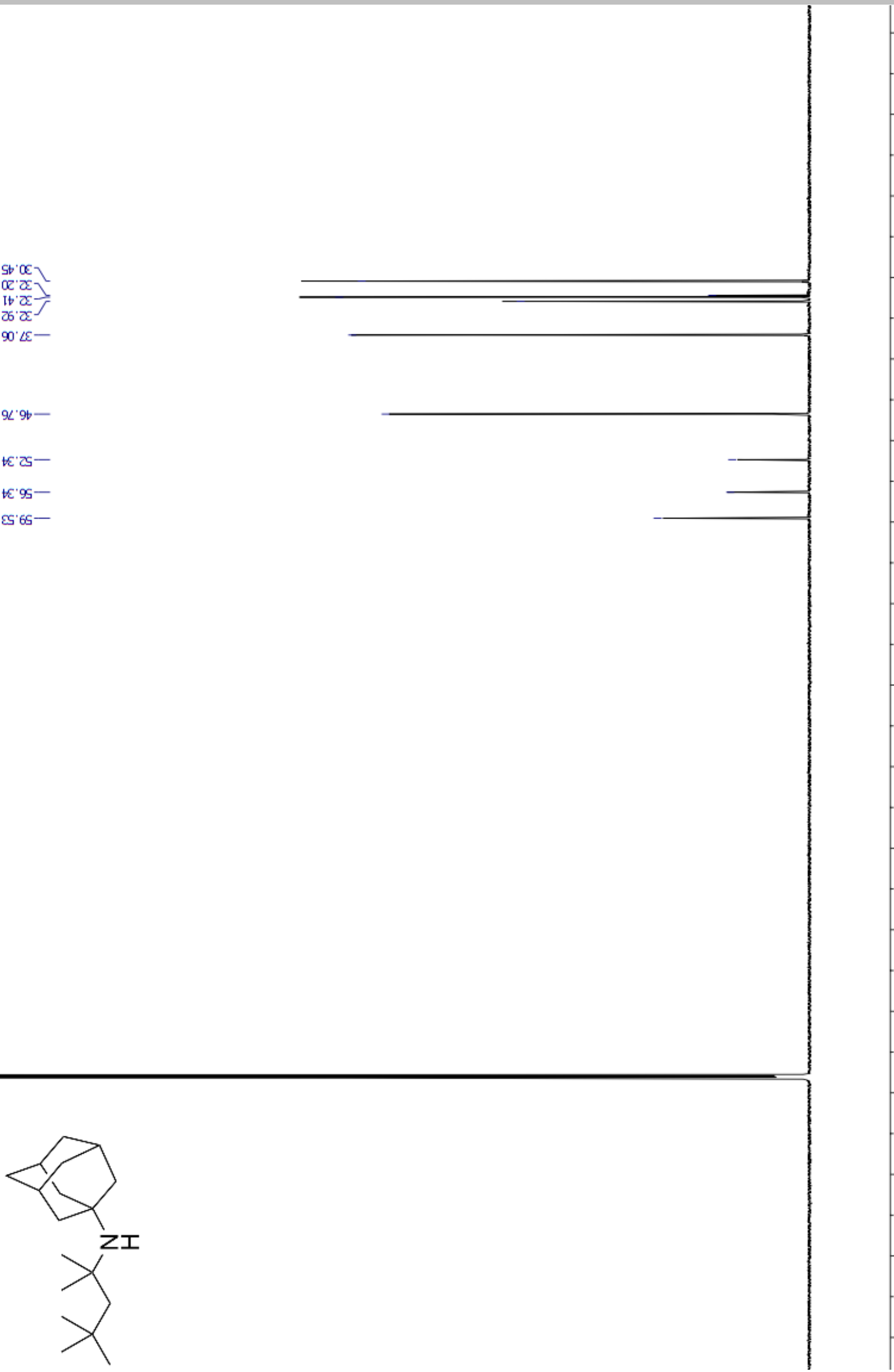

Figure S-2: ${ }^{13} \mathrm{C}$ NMR spectrum of $\mathrm{N}$-(1-adamantyl)-tert-octylamine in $\mathrm{C}_{6} \mathrm{D}_{6}$. 


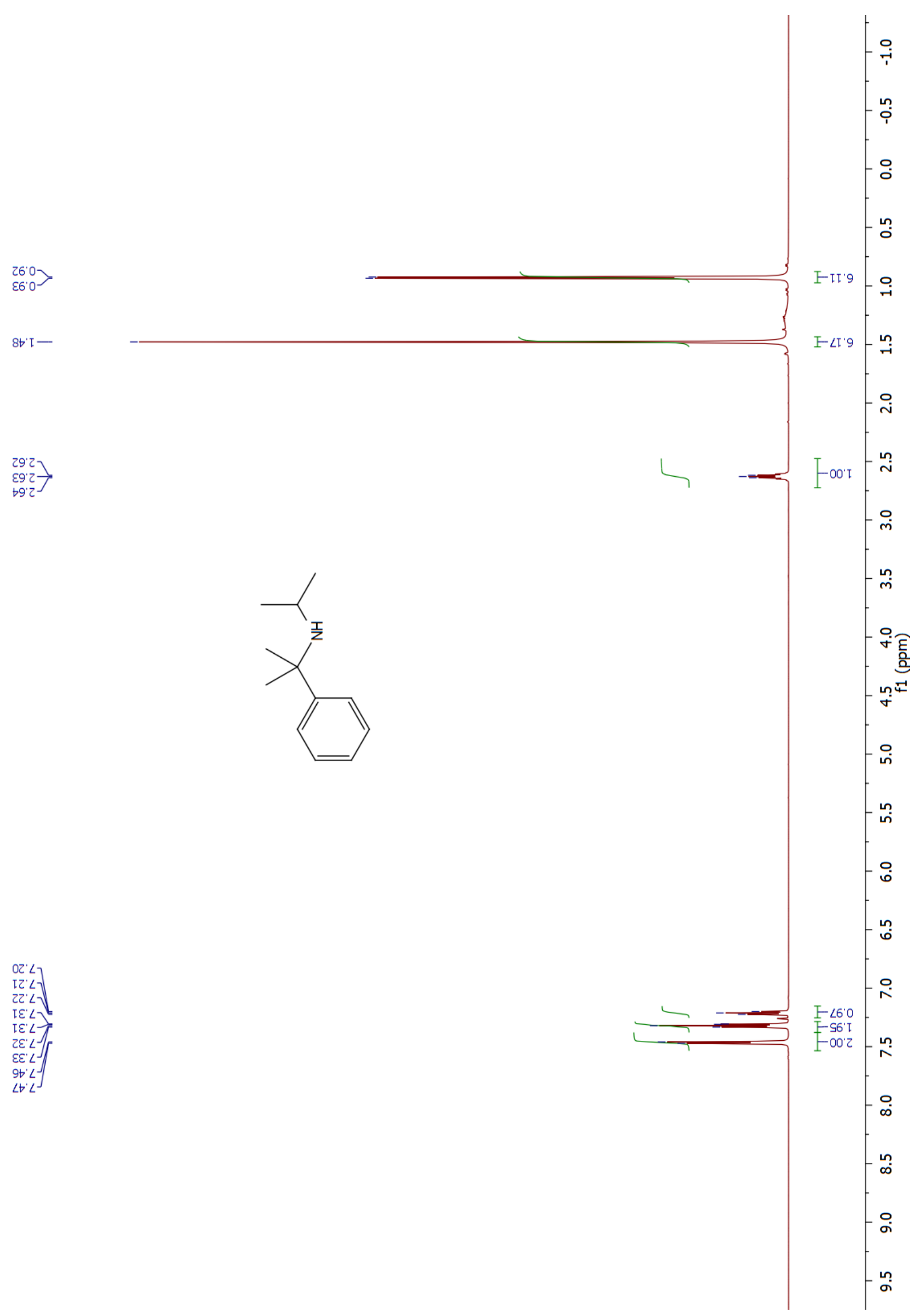

Figure S-3: ${ }^{1} \mathrm{H}$ NMR spectrum of $\mathrm{N}$-isopropylcumylamine in $\mathrm{CDCl}_{3}$. 


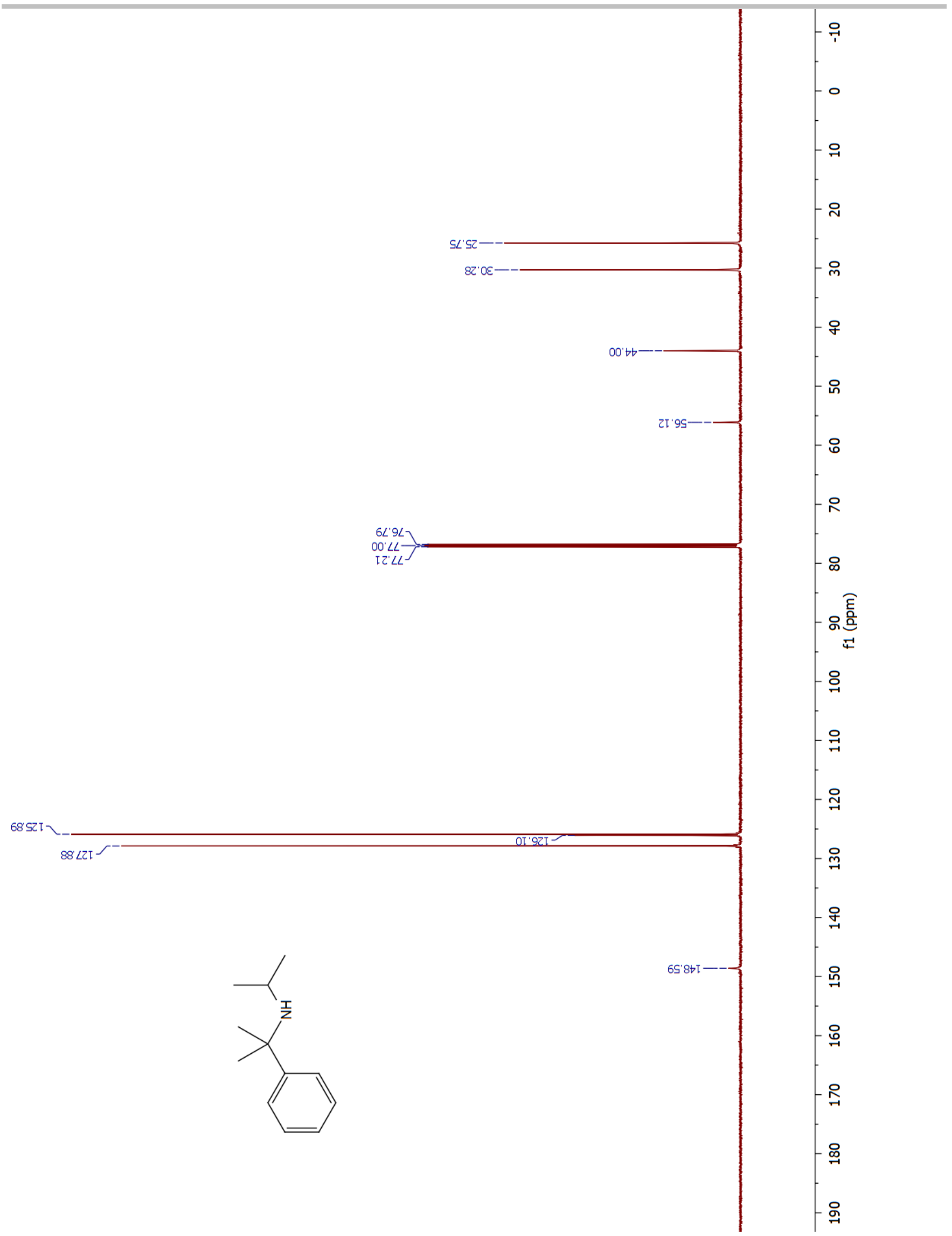

Figure S-4: ${ }^{13} \mathrm{C}\left\{{ }^{1} \mathrm{H}\right\}$ NMR spectrum of $N$-isopropylcumylamine in $\mathrm{CDCl}_{3}$. 


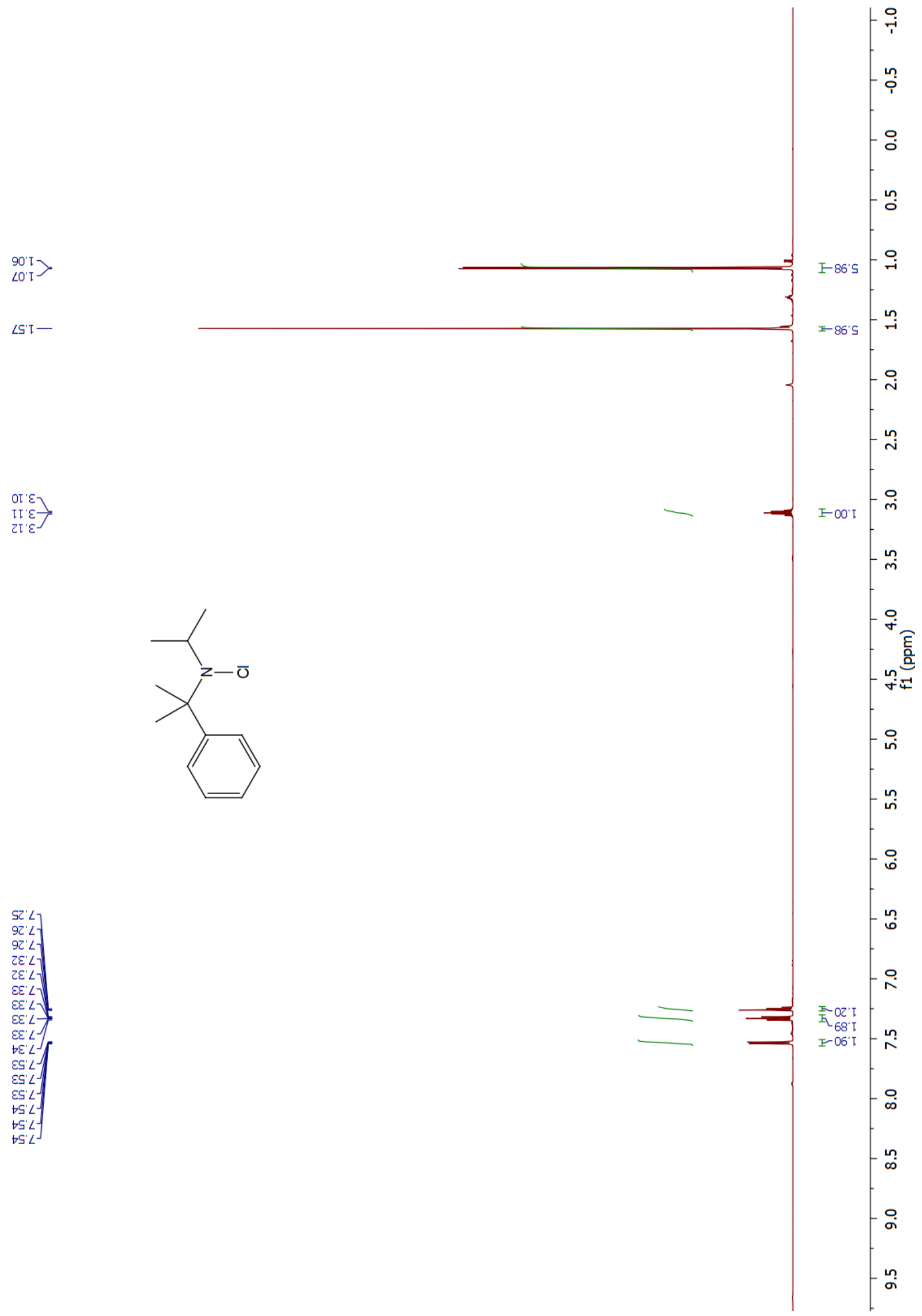

Figure S-5: ${ }^{1} \mathrm{H}$ NMR spectrum of $N$-isopropyl- $N$-chlorocumylamine in $\mathrm{CDCl}_{3}$. 


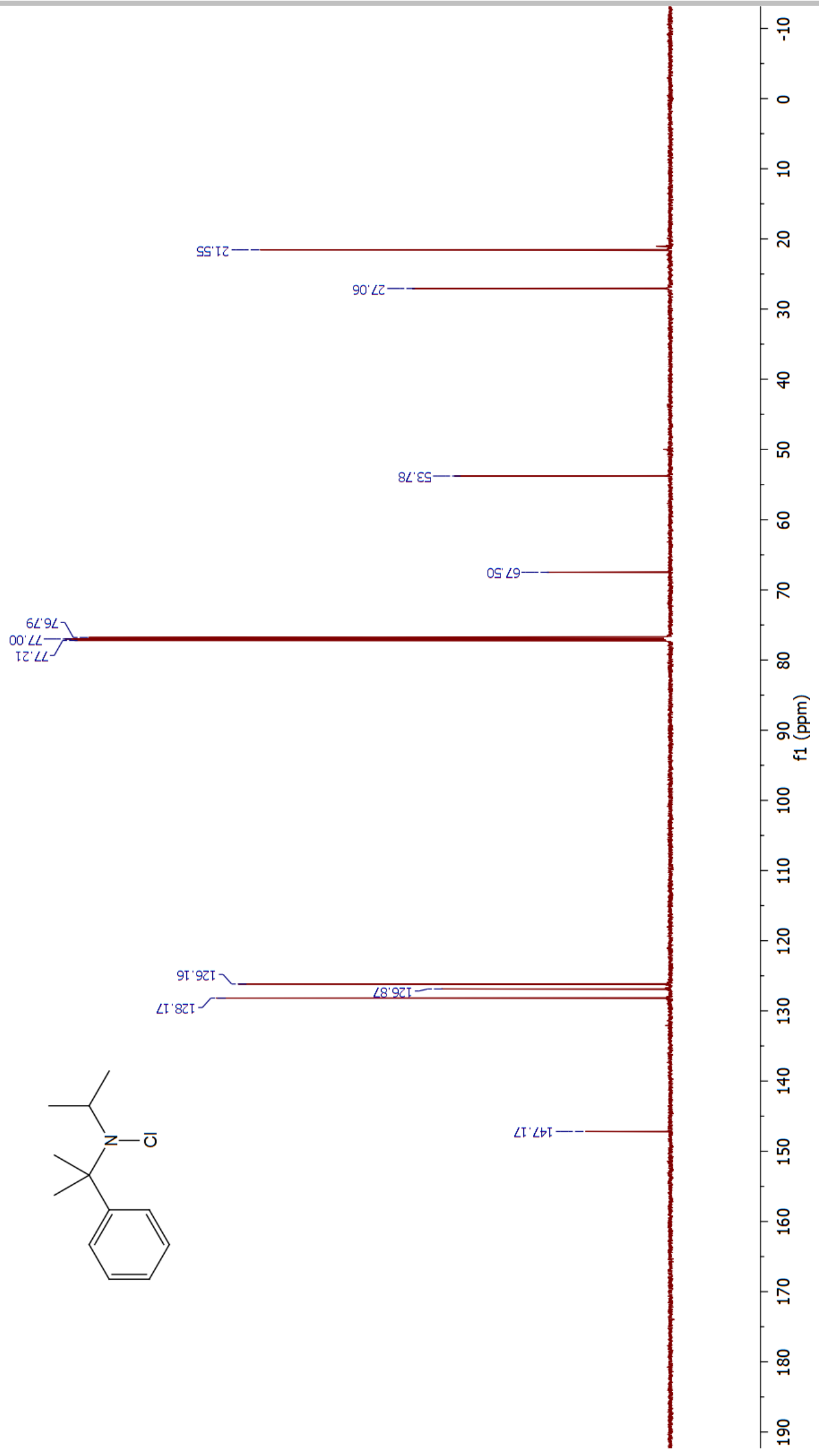

Figure S-6: ${ }^{13} \mathrm{C}\left\{{ }^{1} \mathrm{H}\right\}$ NMR spectrum of $N$-isopropyl- $N$-chlorocumylamine in $\mathrm{CDCl}_{3}$. 


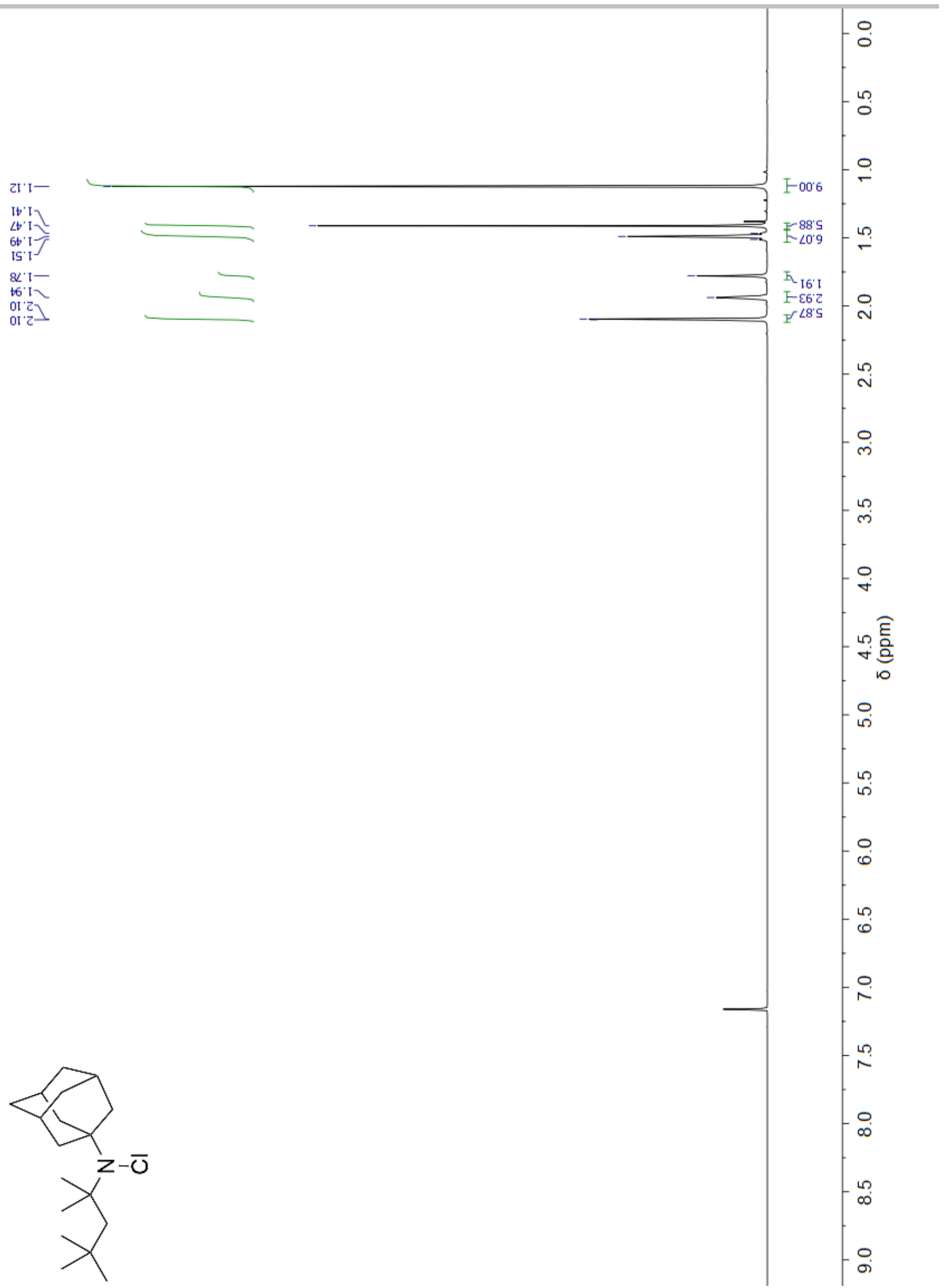

Figure S-7: ${ }^{1} \mathrm{H}$ NMR spectrum of $\mathrm{N}$-(1-adamantyl)- $N$-chloro-tert-octylamine in $\mathrm{C}_{6} \mathrm{D}_{6}$. 
$\sigma^{\circ} \propto$
$U^{\prime} โ \mathcal{E}$

$\infty, \propto \frac{1}{2}$

tI' $x$

$\angle \cdot x-$

$\approx{ }^{\prime} \varepsilon-$

I6.tS-

18' 99

$68.69-$

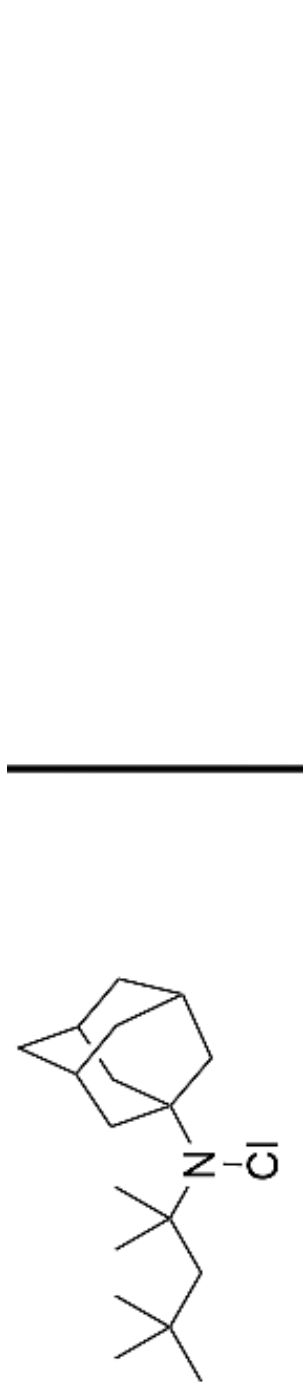

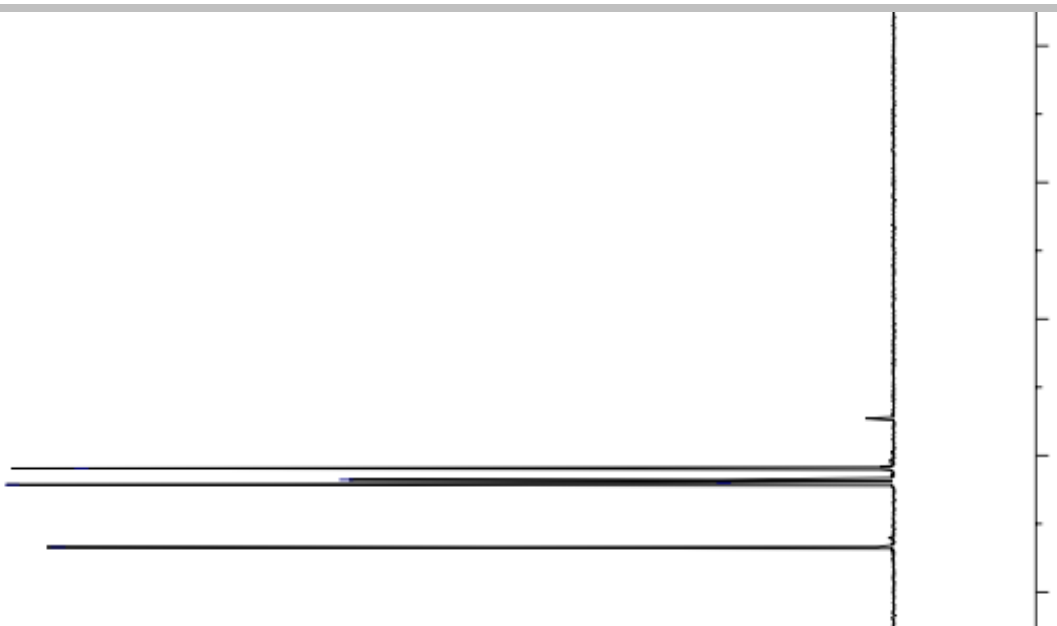

$\circ$

음

ㅇ

요

우

오

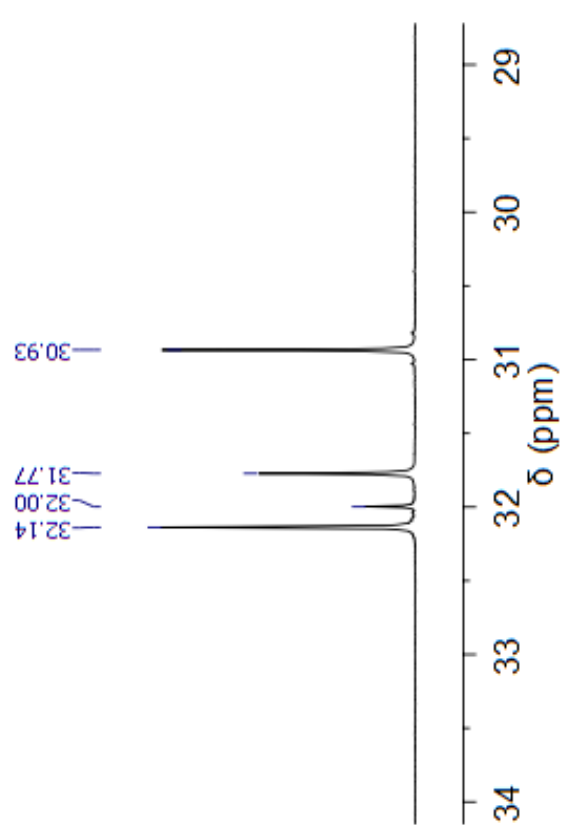

Figure S-8: ${ }^{13} \mathrm{C}\left\{{ }^{1} \mathrm{H}\right\}$ NMR spectrum of $N$-(1-adamantyl)-N-chloro-tert-octylamine in $\mathrm{C}_{6} \mathrm{D}_{6}$. 


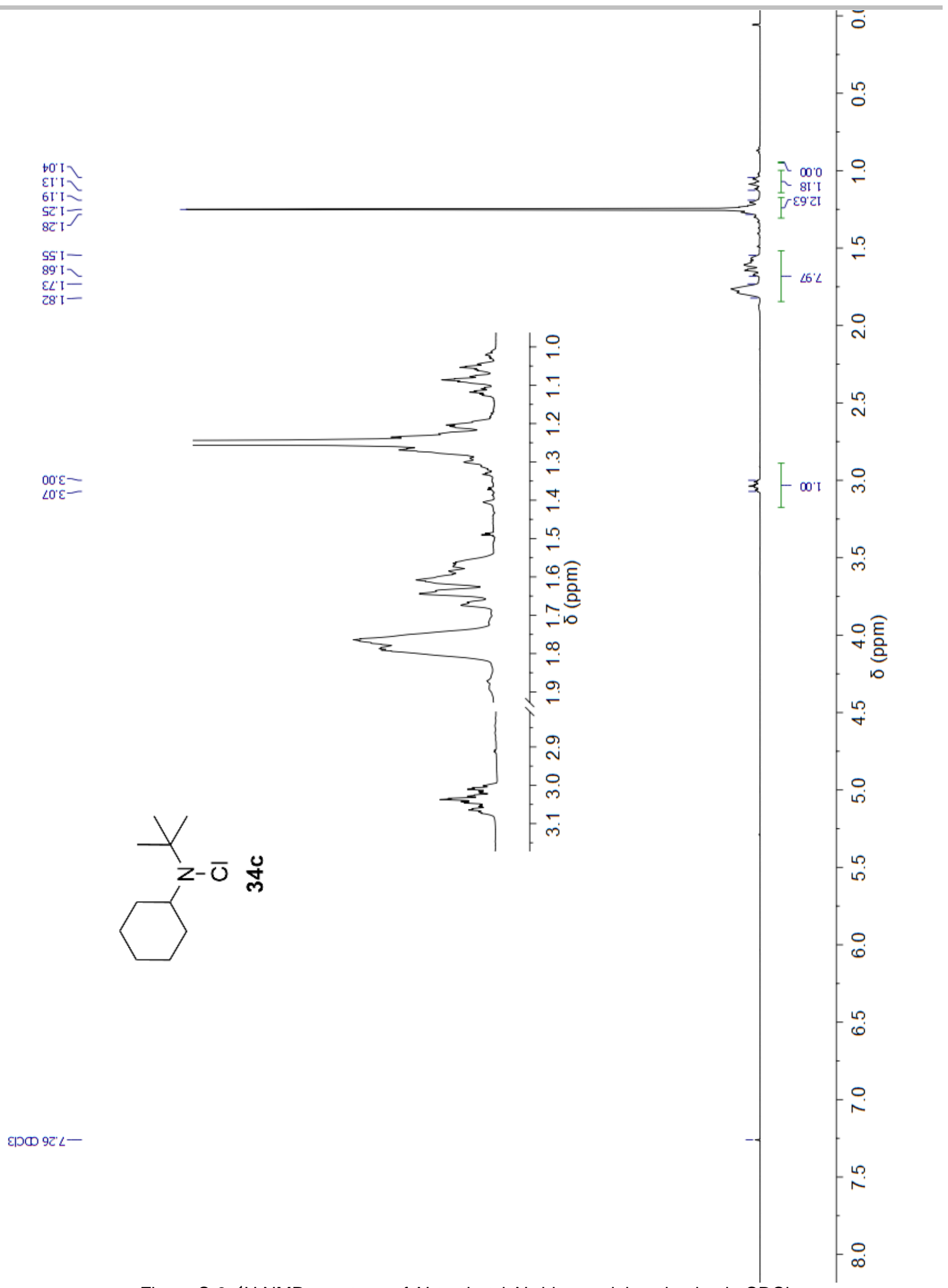

Figure S-9: ${ }^{1} \mathrm{H}$ NMR spectrum of $N$-tert-butyl- $N$-chlorocyclohexylamine in $\mathrm{CDCl}_{3}$. 


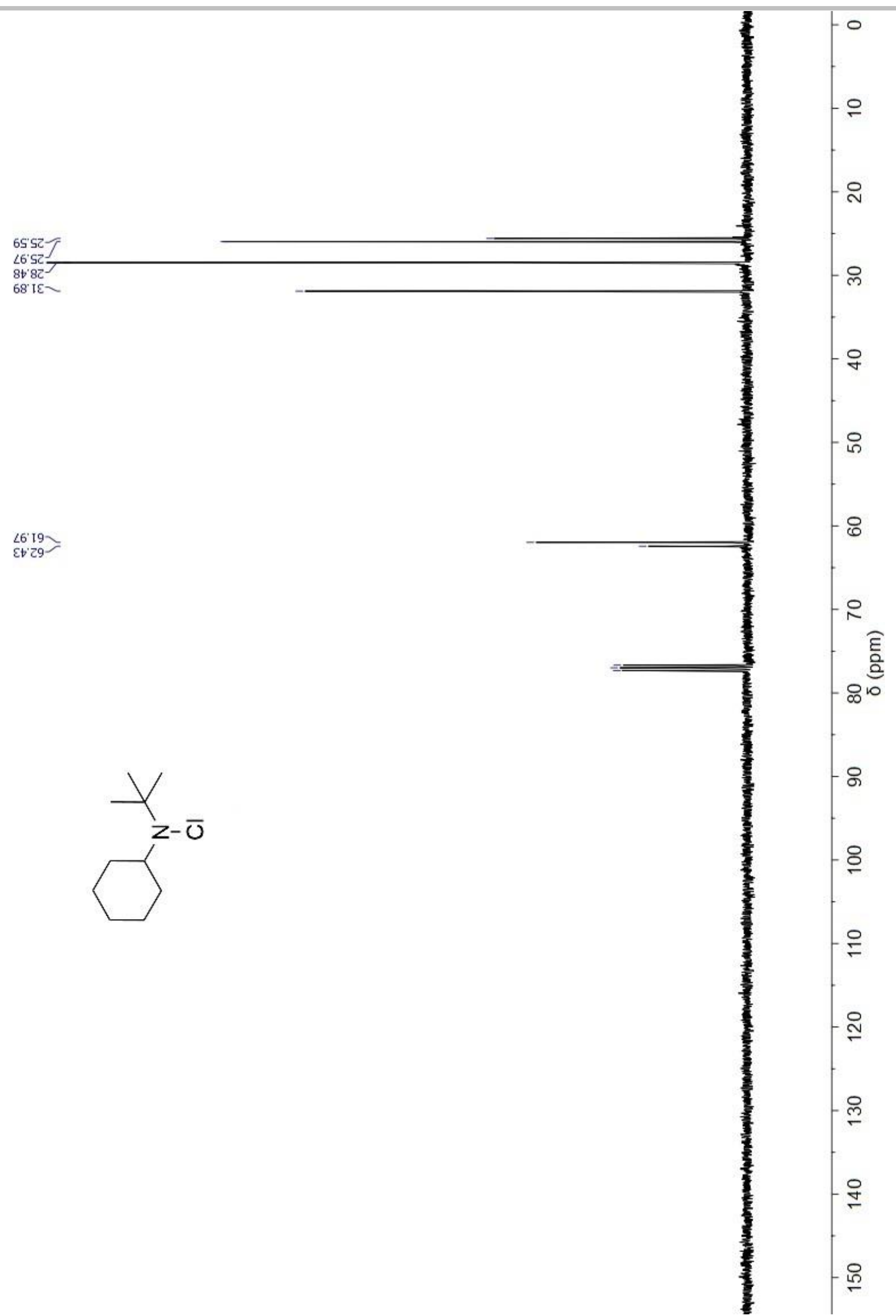

Figure S-10: ${ }^{13} \mathrm{C}\left\{{ }^{1} \mathrm{H}\right\}$ NMR spectrum of $N$-tert-butyl- $N$-chlorocyclohexylamine in $\mathrm{CDCl}_{3}$. 


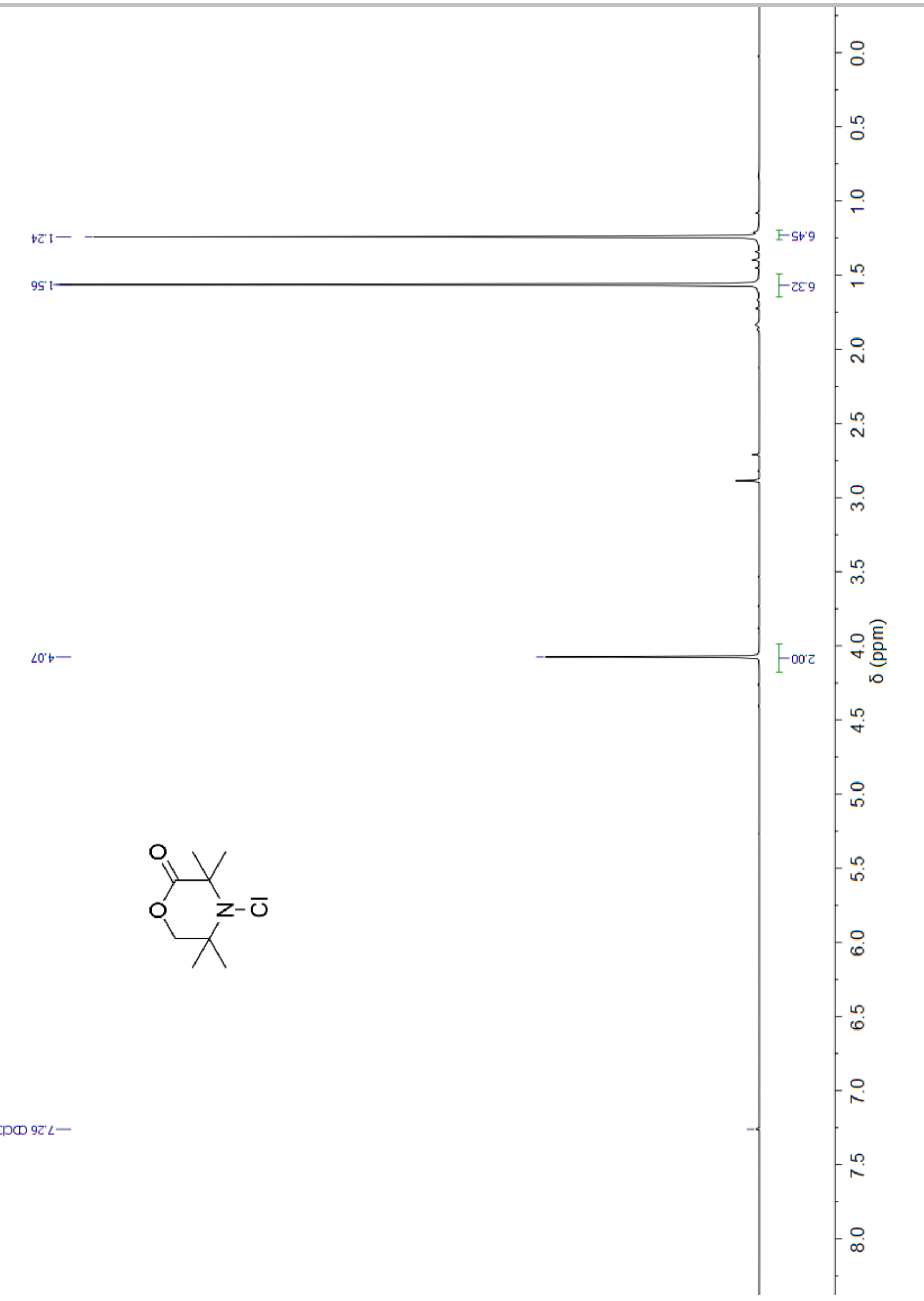

Figure S-11: ${ }^{1} \mathrm{H}$ NMR spectrum of 4-chloro-3,3,5,5-tetramethylmorpholin-2-one in $\mathrm{CDCl}_{3}$. 


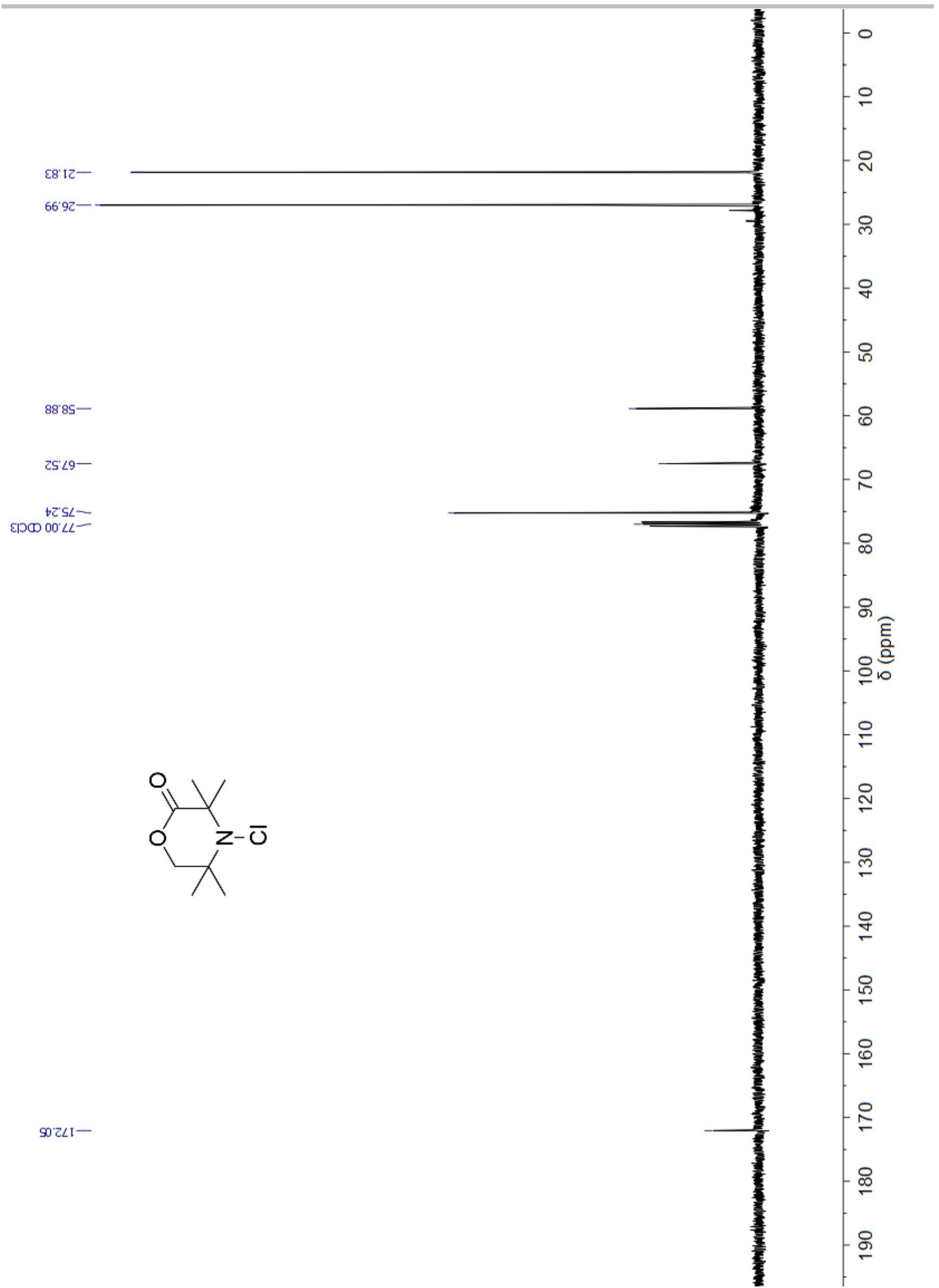

Figure S-12: ${ }^{13} \mathrm{C}\left\{{ }^{1} \mathrm{H}\right\}$ NMR spectrum of 4-chloro-3,3,5,5-tetramethylmorpholin-2-one in $\mathrm{CDCl}_{3}$. 


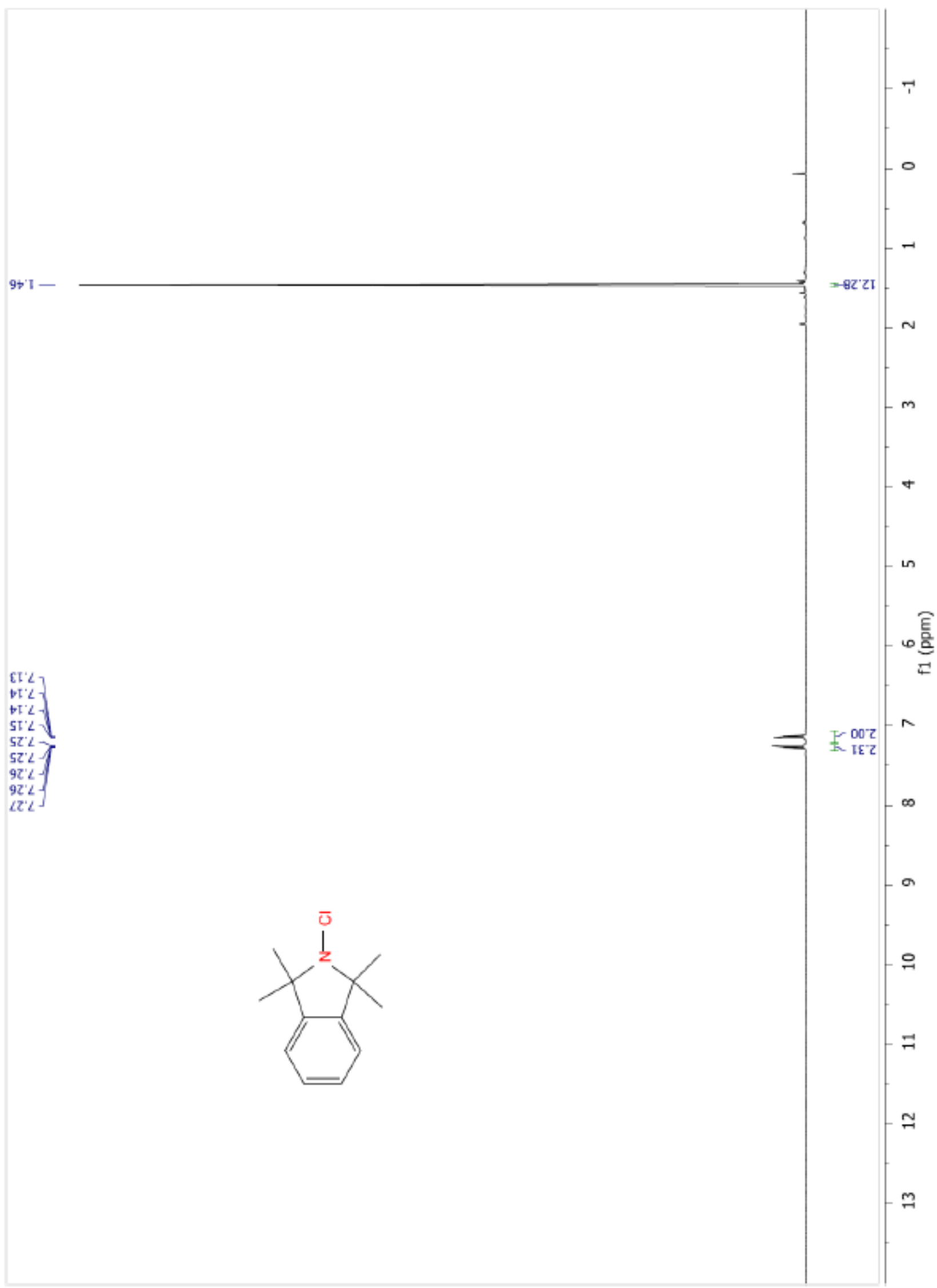

Figure S-13: ${ }^{1} \mathrm{H}$ NMR spectrum of 2-chloro-1,1,3,3-tetramethylisoindoline in $\mathrm{CDCl}_{3}$. 


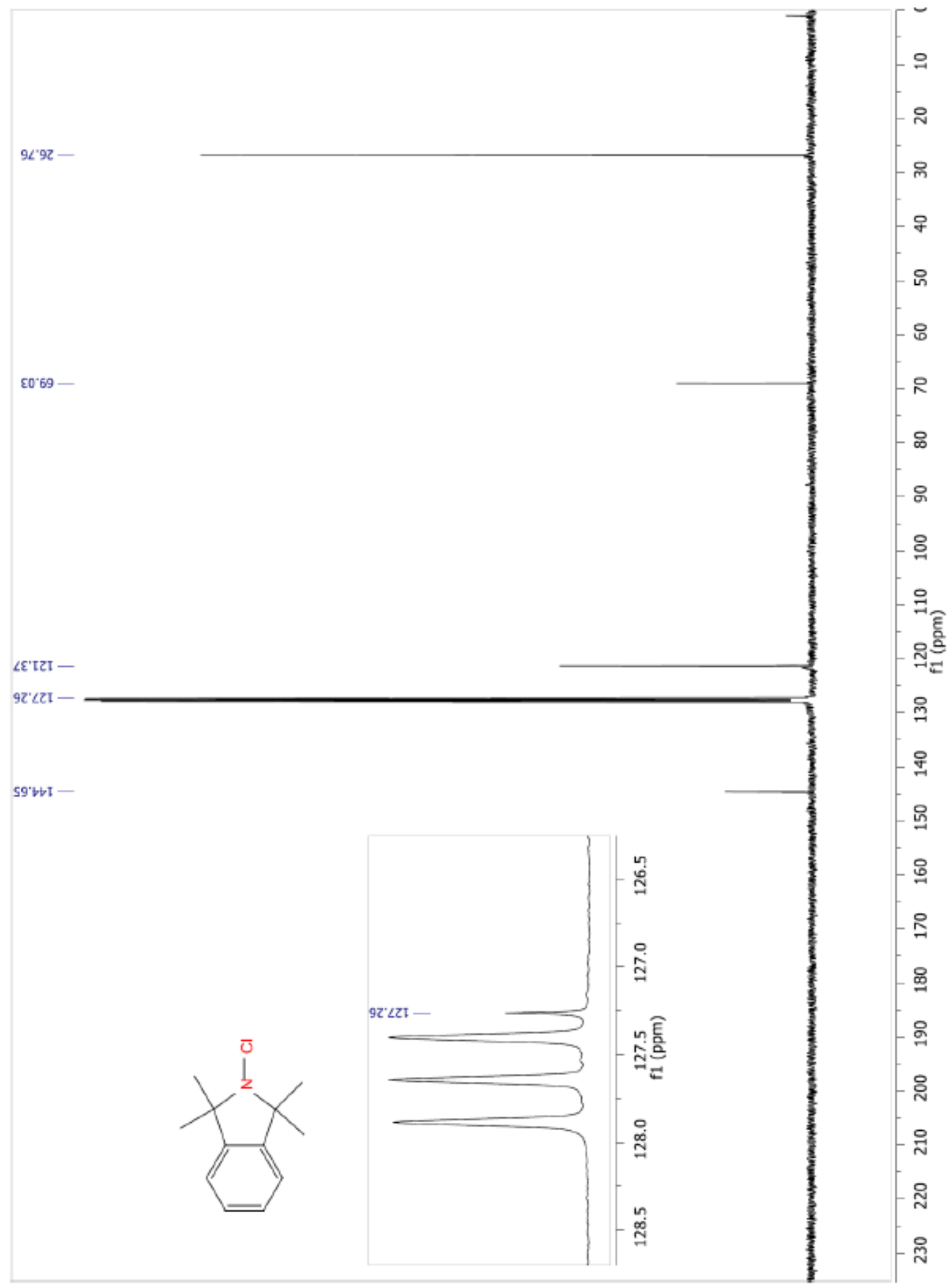

Figure S-14: ${ }^{13} \mathrm{C}\left\{{ }^{1} \mathrm{H}\right\}$ NMR spectrum of 2-chloro-1,1,3,3-tetramethylisoindoline in $\mathrm{C}_{6} \mathrm{D}_{6}$. 


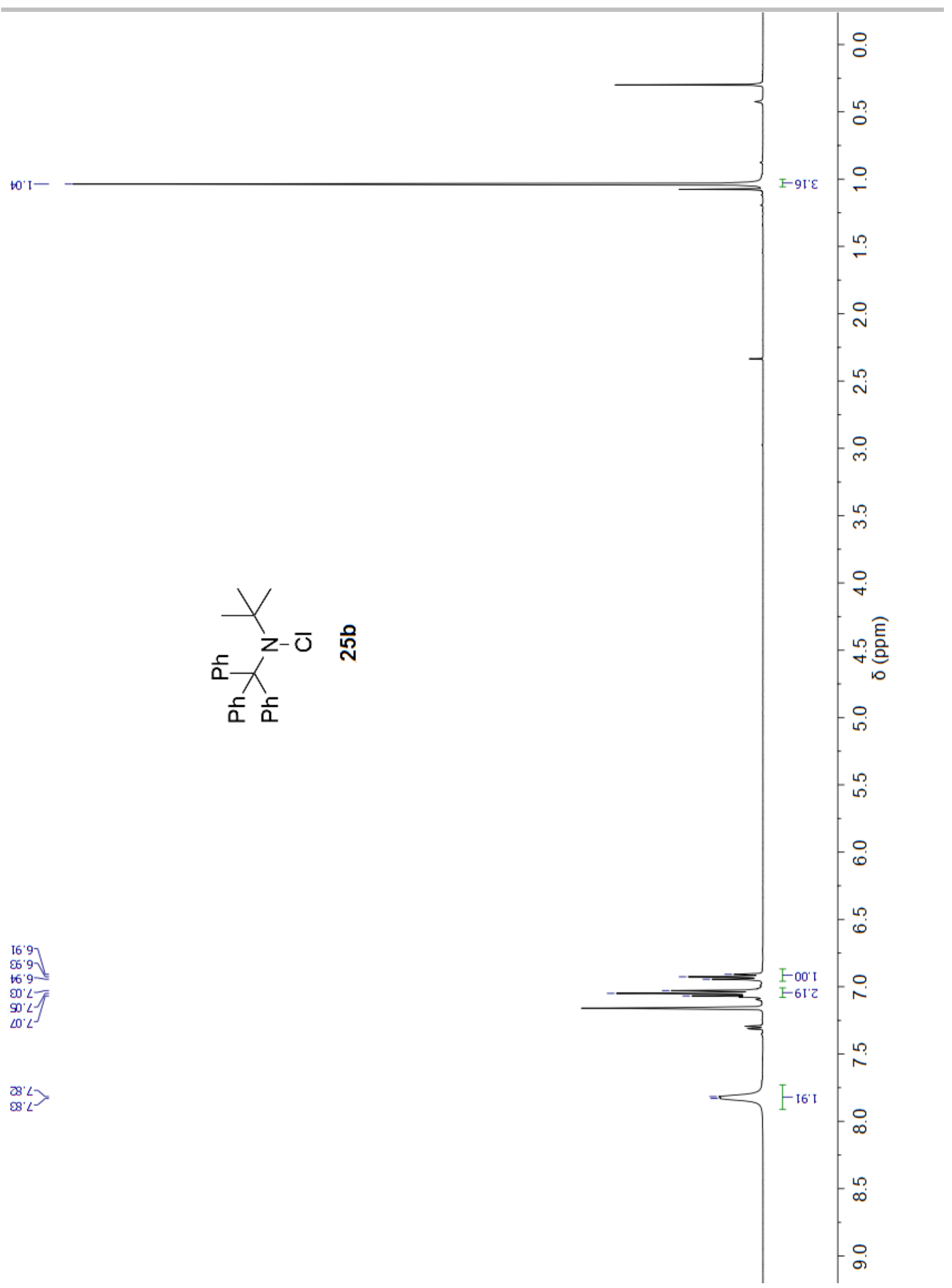

Figure S-15: ${ }^{1} \mathrm{H}$ NMR spectrum of $\mathbf{2 5 b}$ in $\mathrm{C}_{6} \mathrm{D}_{6}$. 


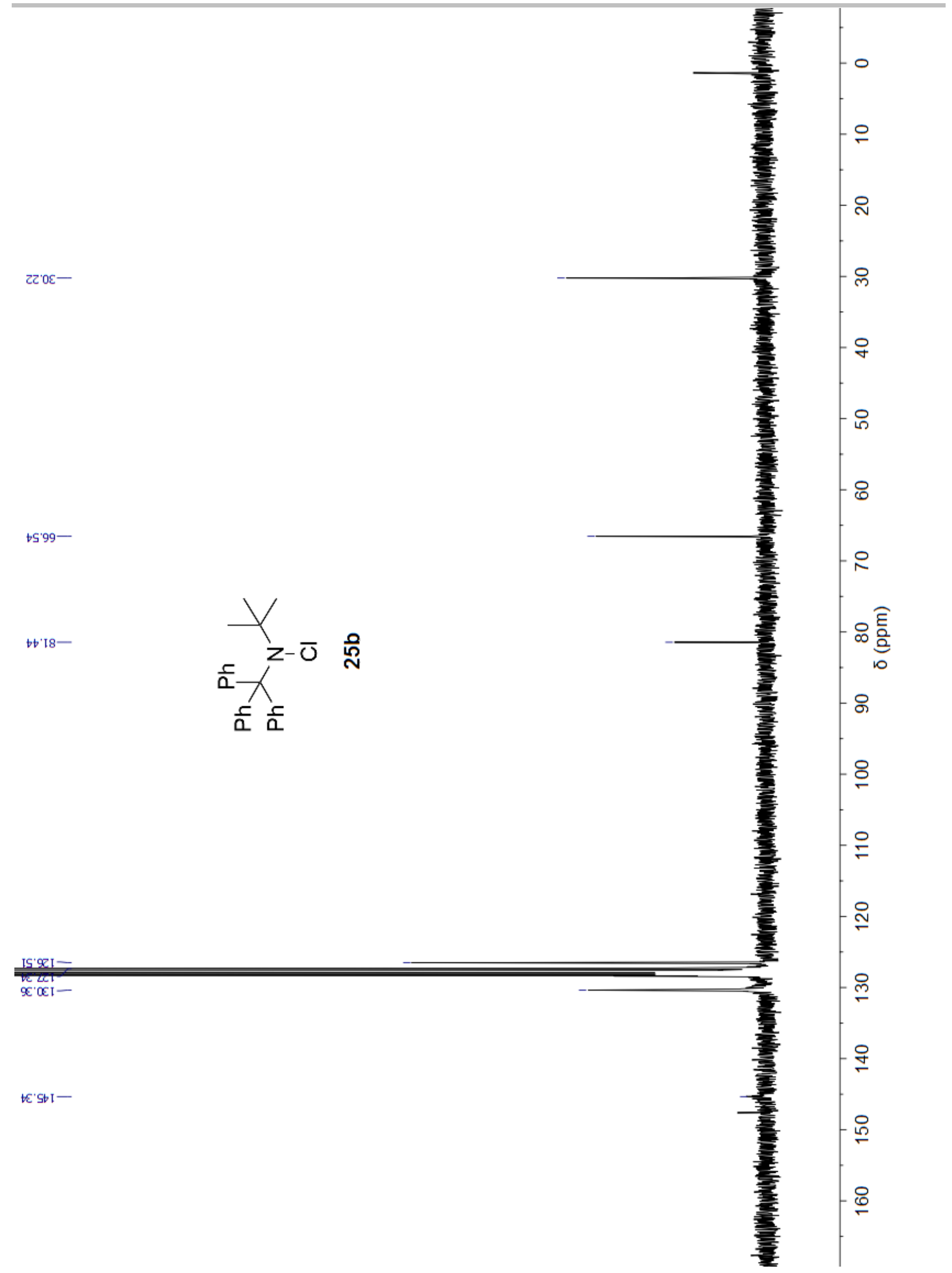

Figure $\mathrm{S}-16:{ }^{13} \mathrm{C}\left\{{ }^{1} \mathrm{H}\right\}$ NMR spectrum of $25 \mathrm{~b}$ in $\mathrm{C}_{6} \mathrm{D}_{6}$. 


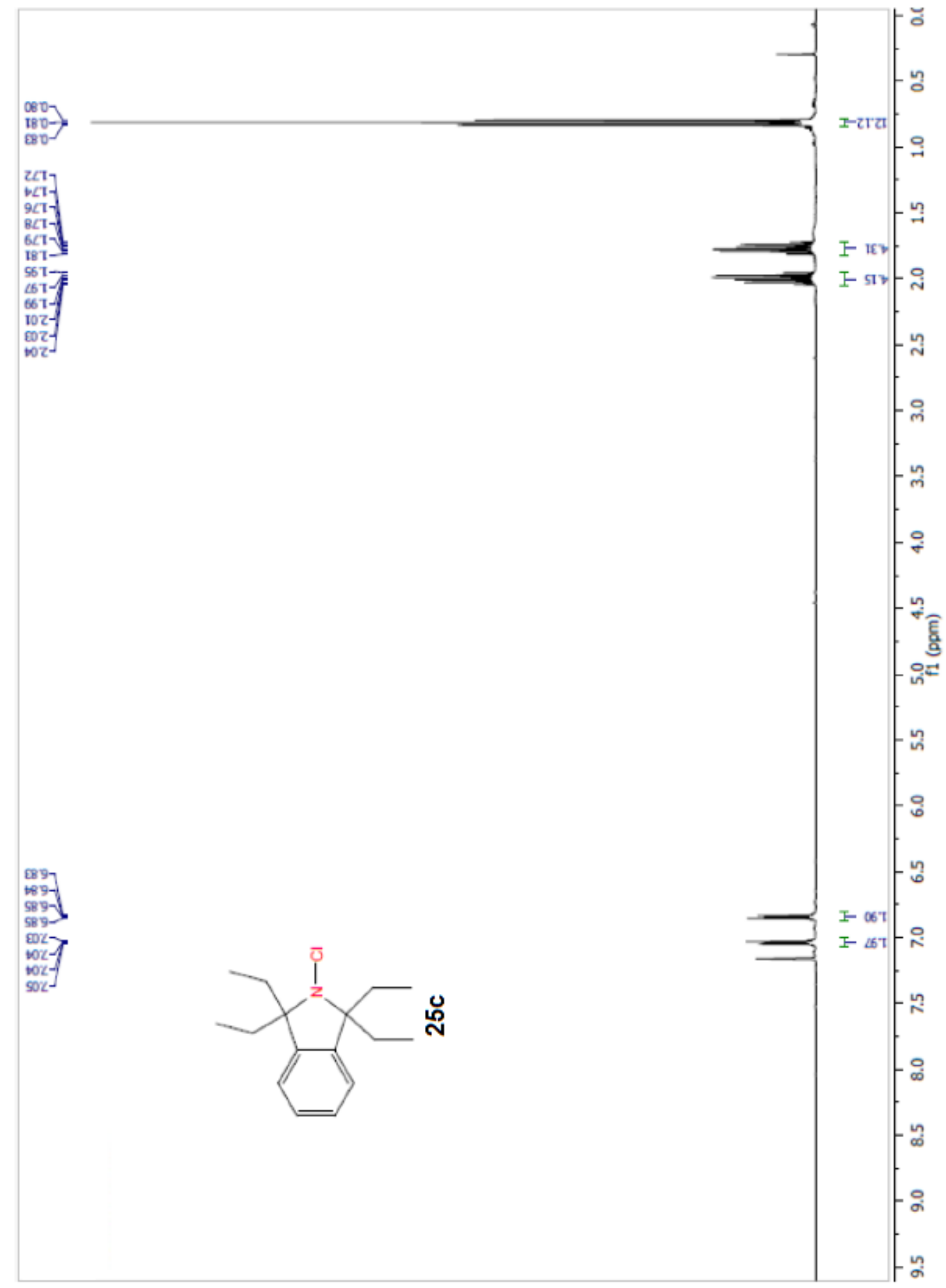

Figure S-17: ${ }^{1} \mathrm{H}$ NMR spectrum of $25 \mathrm{c}$ in $\mathrm{C}_{6} \mathrm{D}_{6}$. 


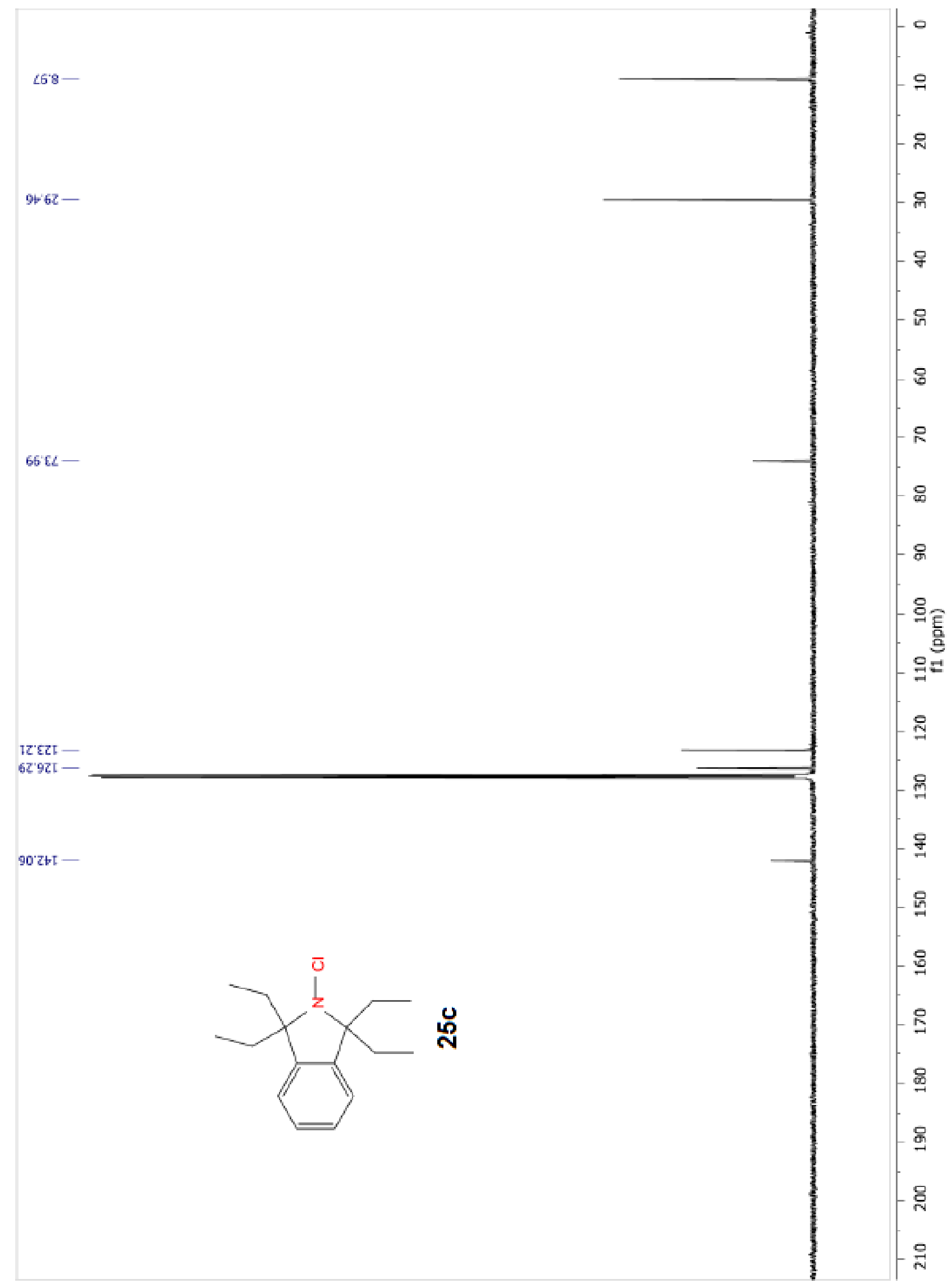

Figure $\mathrm{S}-18:{ }^{13} \mathrm{C}\left\{{ }^{1} \mathrm{H}\right\}$ NMR spectrum of $25 \mathrm{c}$ in $\mathrm{C}_{6} \mathrm{D}_{6}$. 


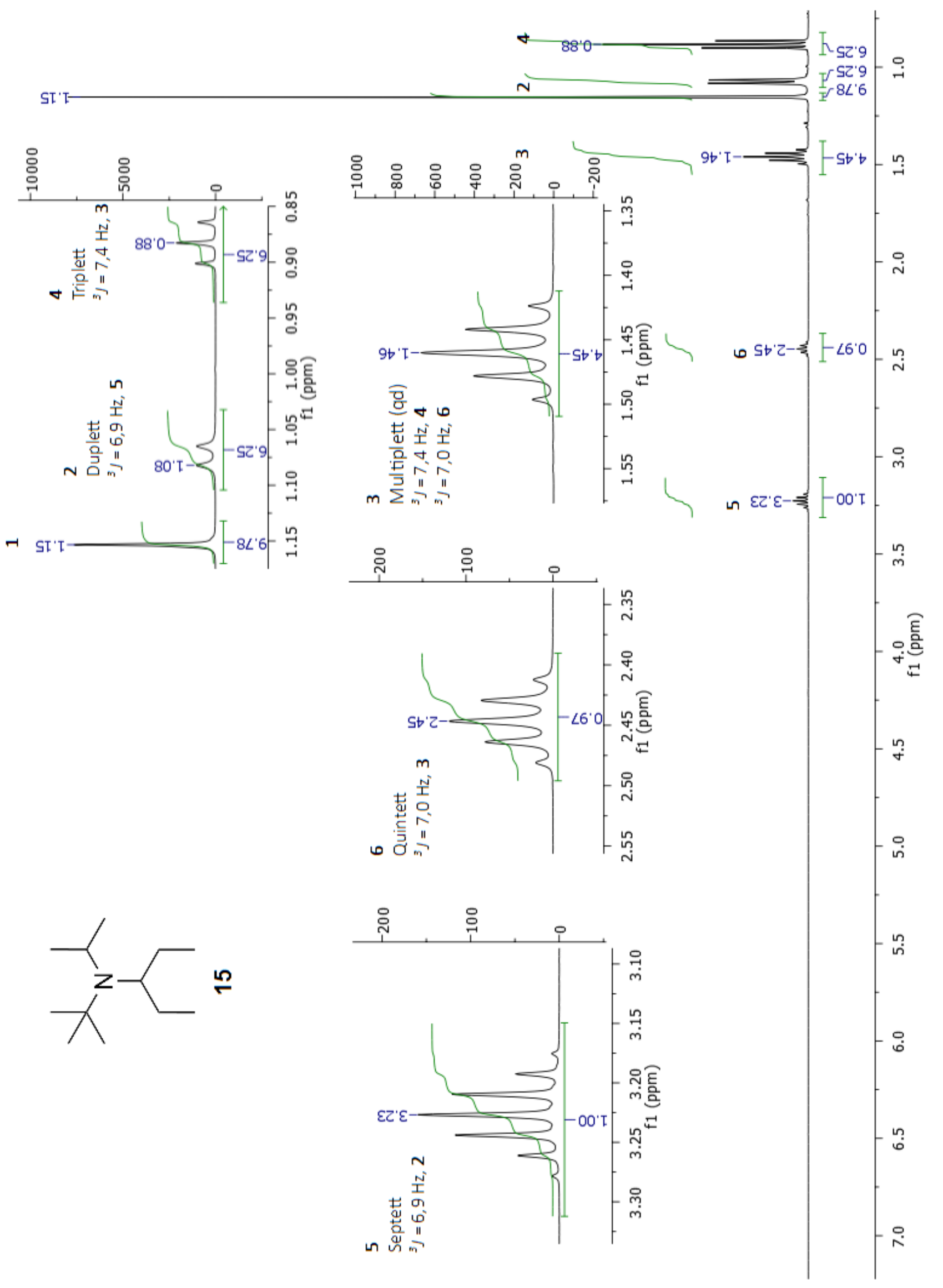

Figure S-19: ${ }^{1} \mathrm{H}$ NMR spectrum of 15 in $\mathrm{CDCl}_{3}$. 


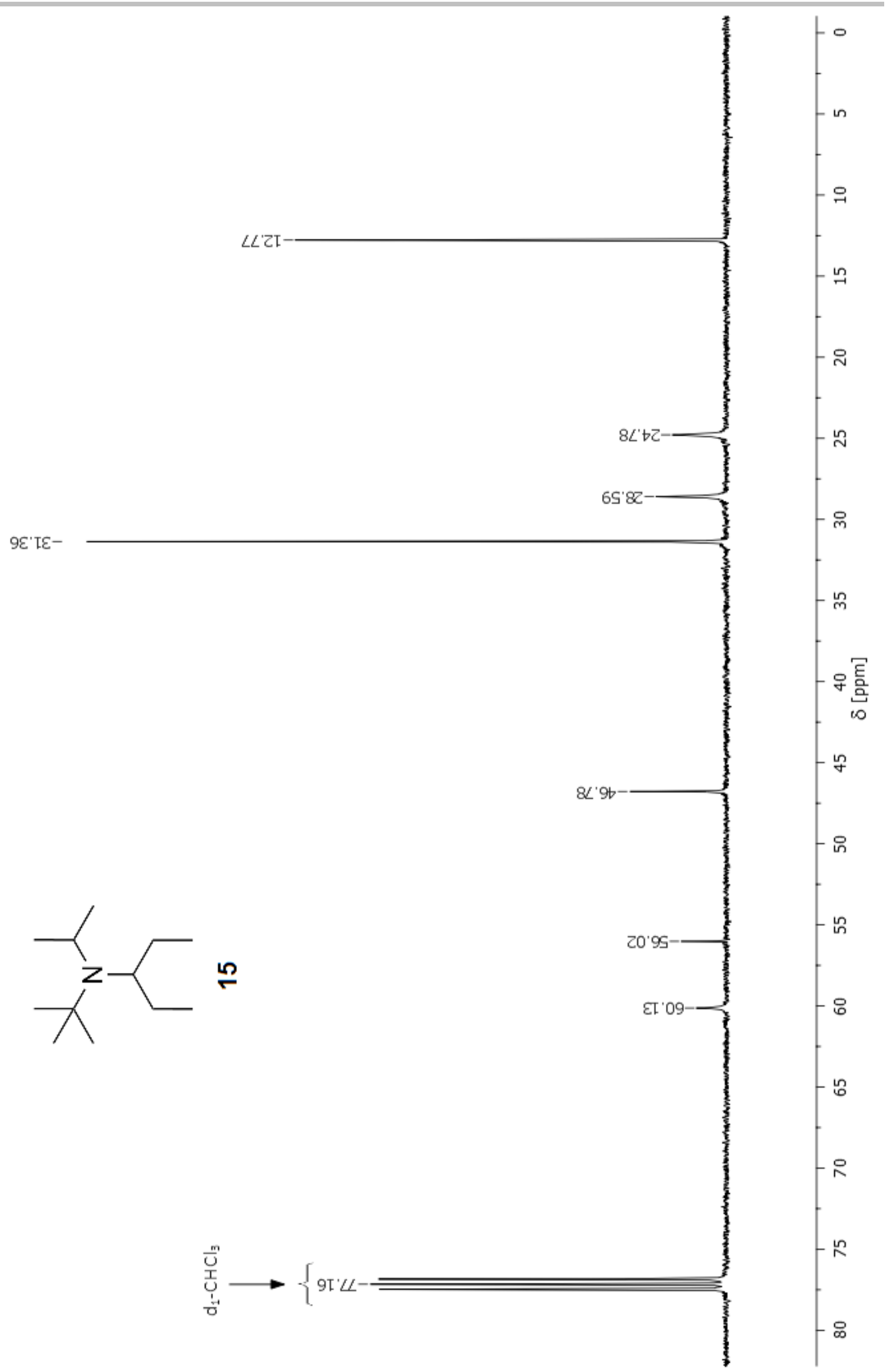

Figure S-20: ${ }^{13} \mathrm{C}\left\{{ }^{1} \mathrm{H}\right\}$ NMR spectrum of 15 in $\mathrm{CDCl}_{3}$. 


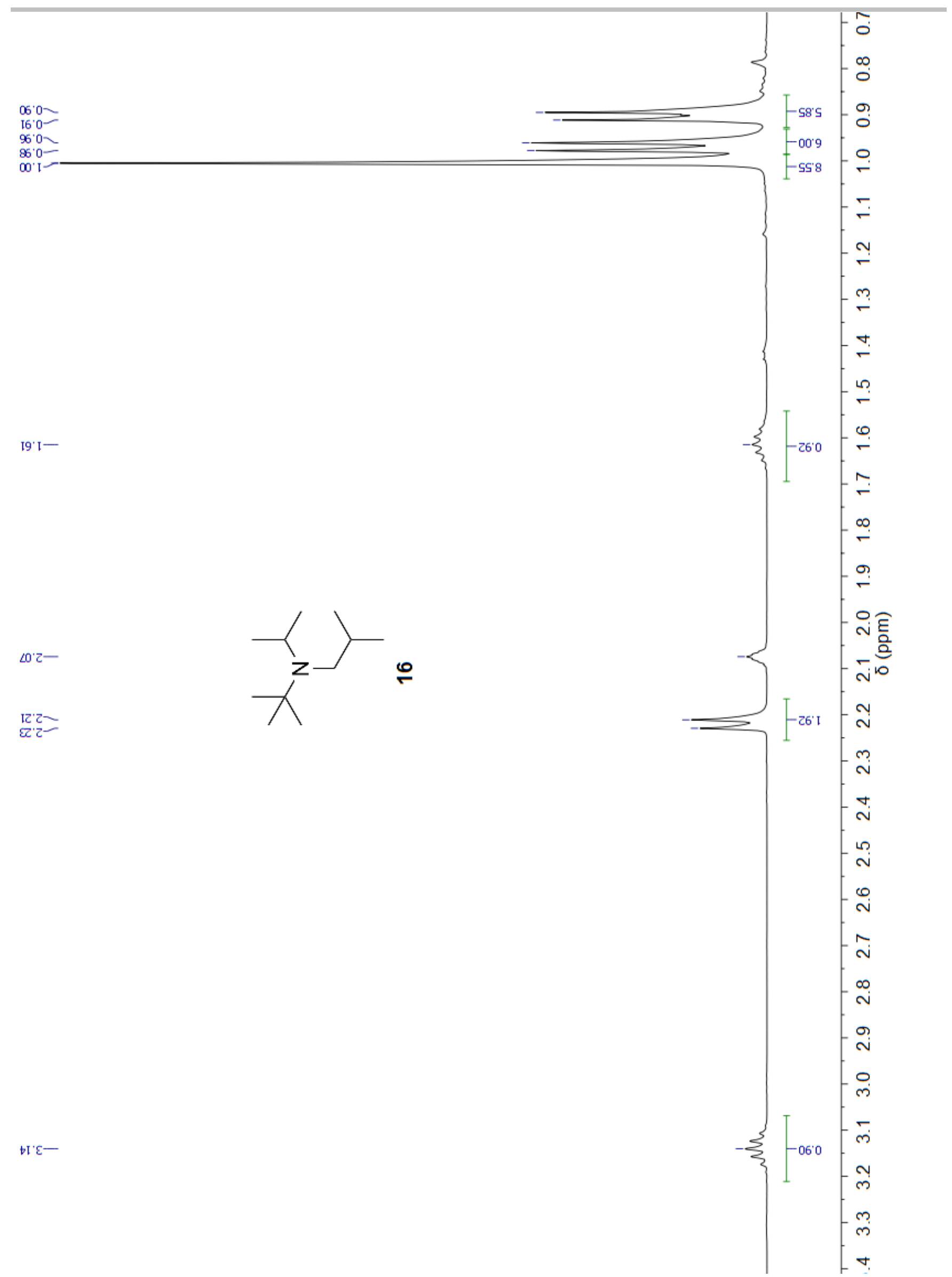

Figure S-21: ${ }^{1} \mathrm{H}$ NMR spectrum of 16 in $\mathrm{C}_{7} \mathrm{D}_{8}$. 
$0 b^{\prime} \propto-$

$88^{\prime} \mathrm{CZ}-$

๗'そ-

I8' 8 -

$\propto ⿻$

$98 \cdot 9 b-$

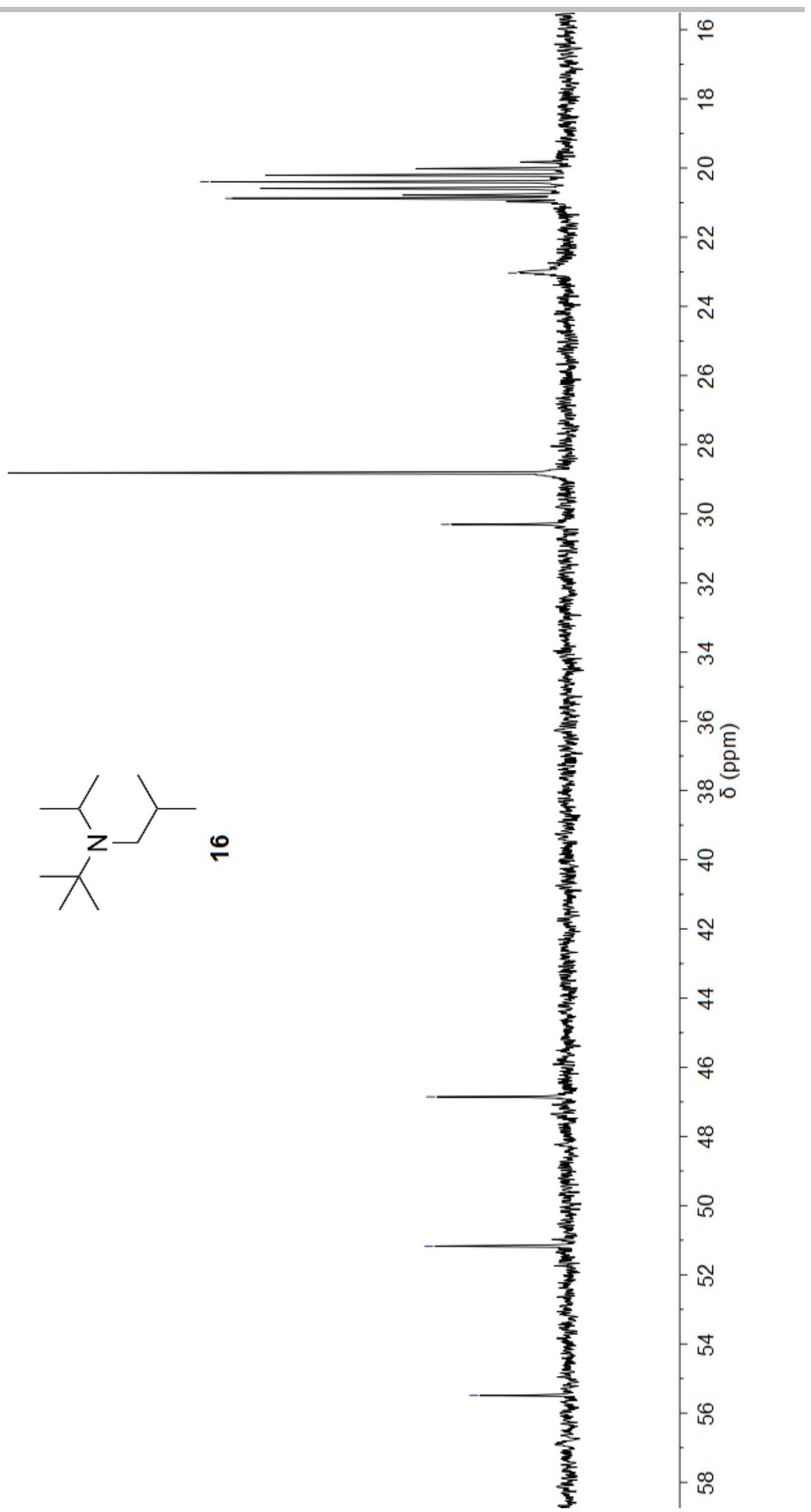

LI'IS-

66'오-

Figure S-22: ${ }^{13} \mathrm{C}\left\{{ }^{1} \mathrm{H}\right\}$ NMR spectrum of 16 in $\mathrm{C}_{7} \mathrm{D}_{8}$. 


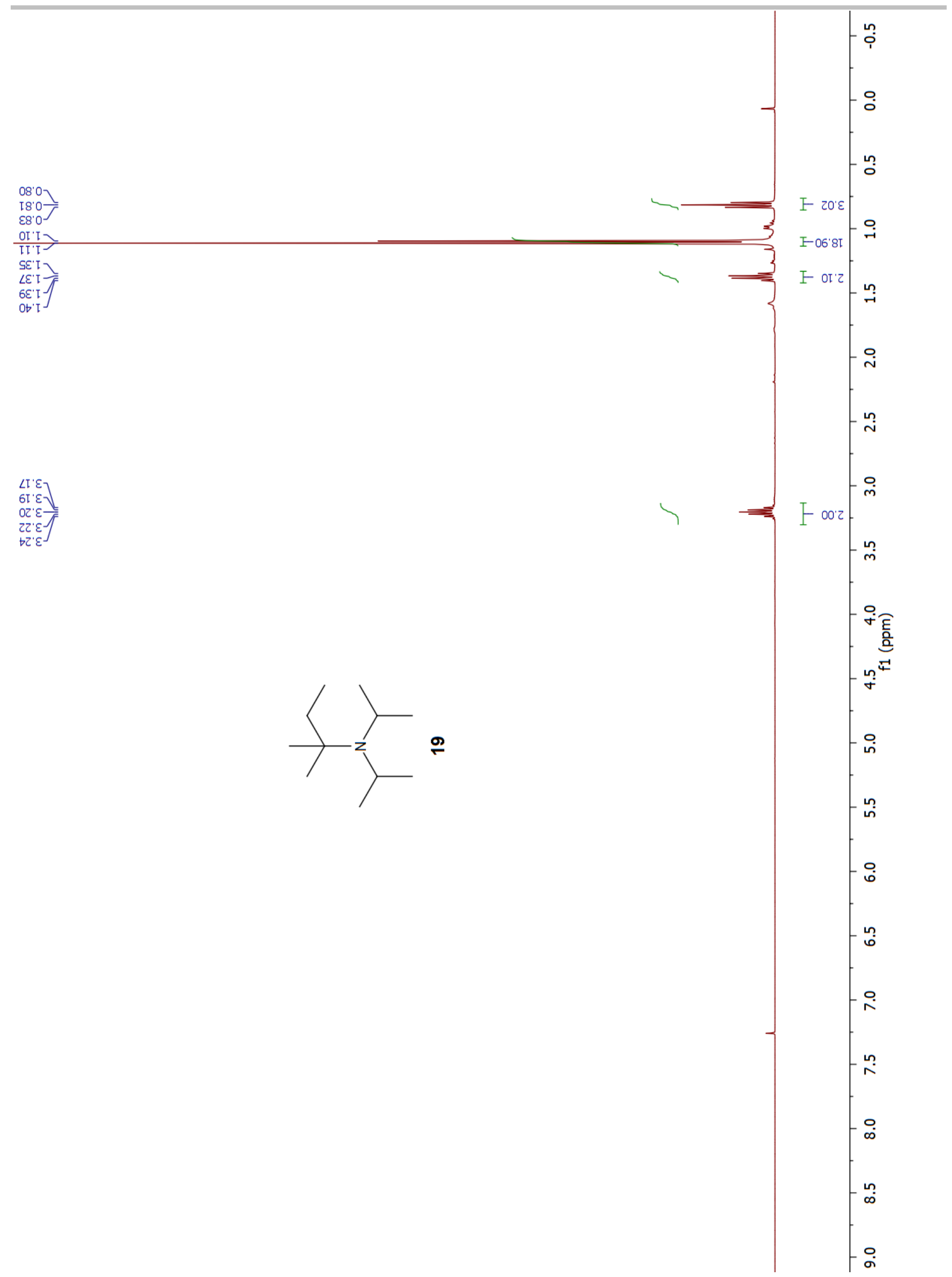

Figure S-23: ${ }^{1} \mathrm{H}$ NMR spectrum of 19 in $\mathrm{CDCl}_{3}$. 
SUPPORTING INFORMATION

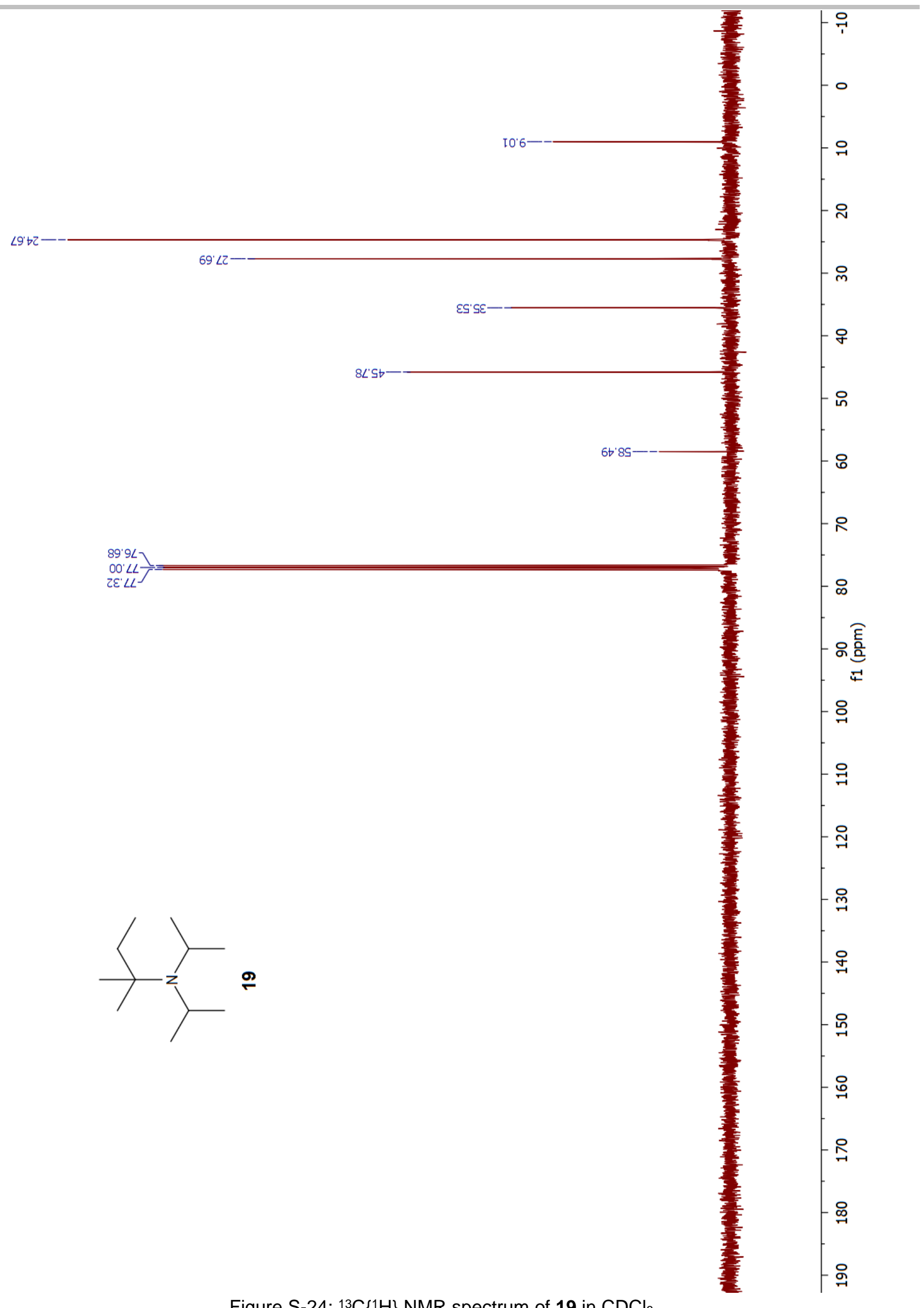

Figure S-24: ${ }^{13} \mathrm{C}\left\{{ }^{1} \mathrm{H}\right\}$ NMR spectrum of 19 in $\mathrm{CDCl}_{3}$.

S-26 


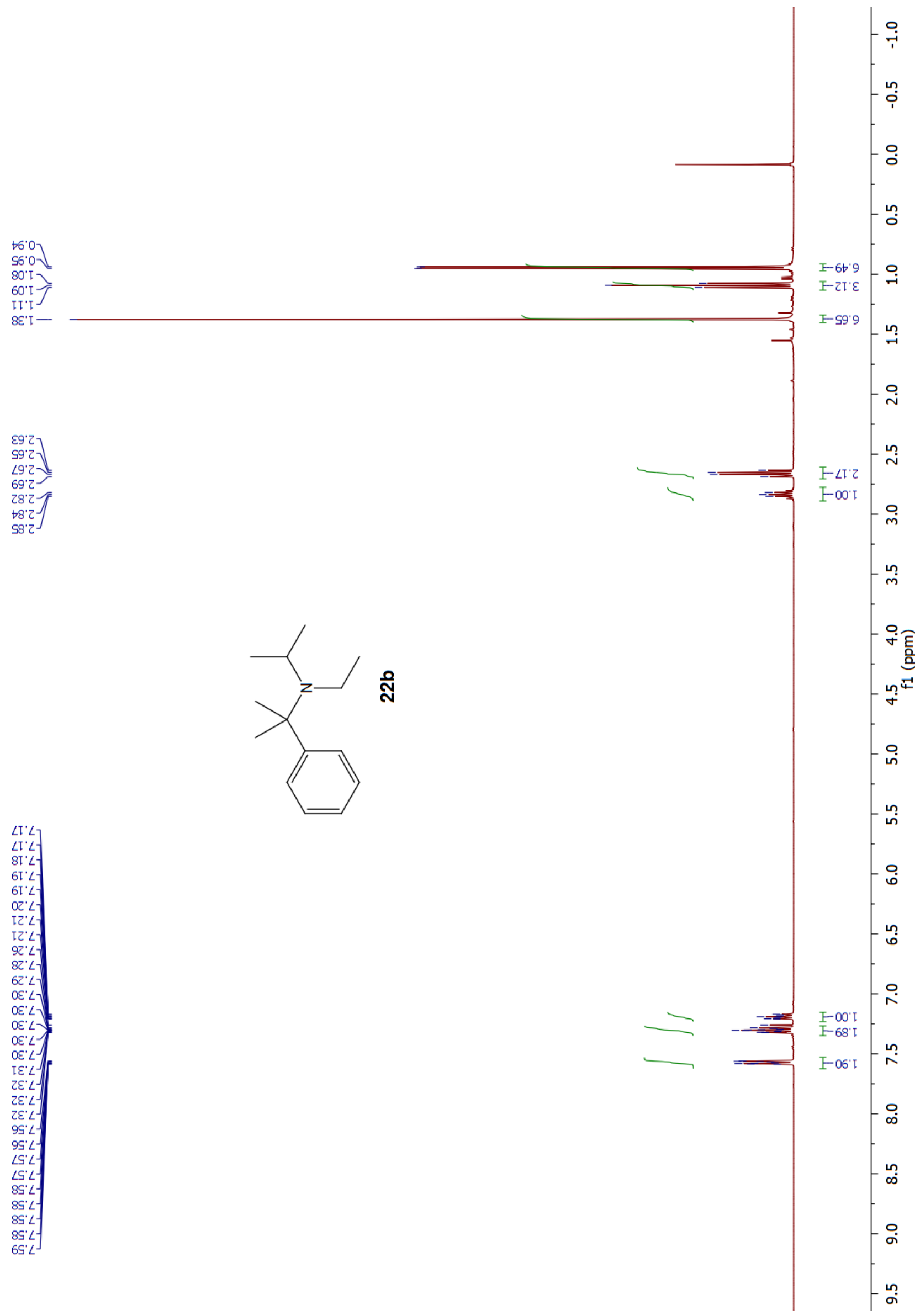

Figure S-25: ${ }^{1} \mathrm{H}$ NMR spectrum of $\mathbf{2 2 b}$ in $\mathrm{CDCl}_{3}$. 


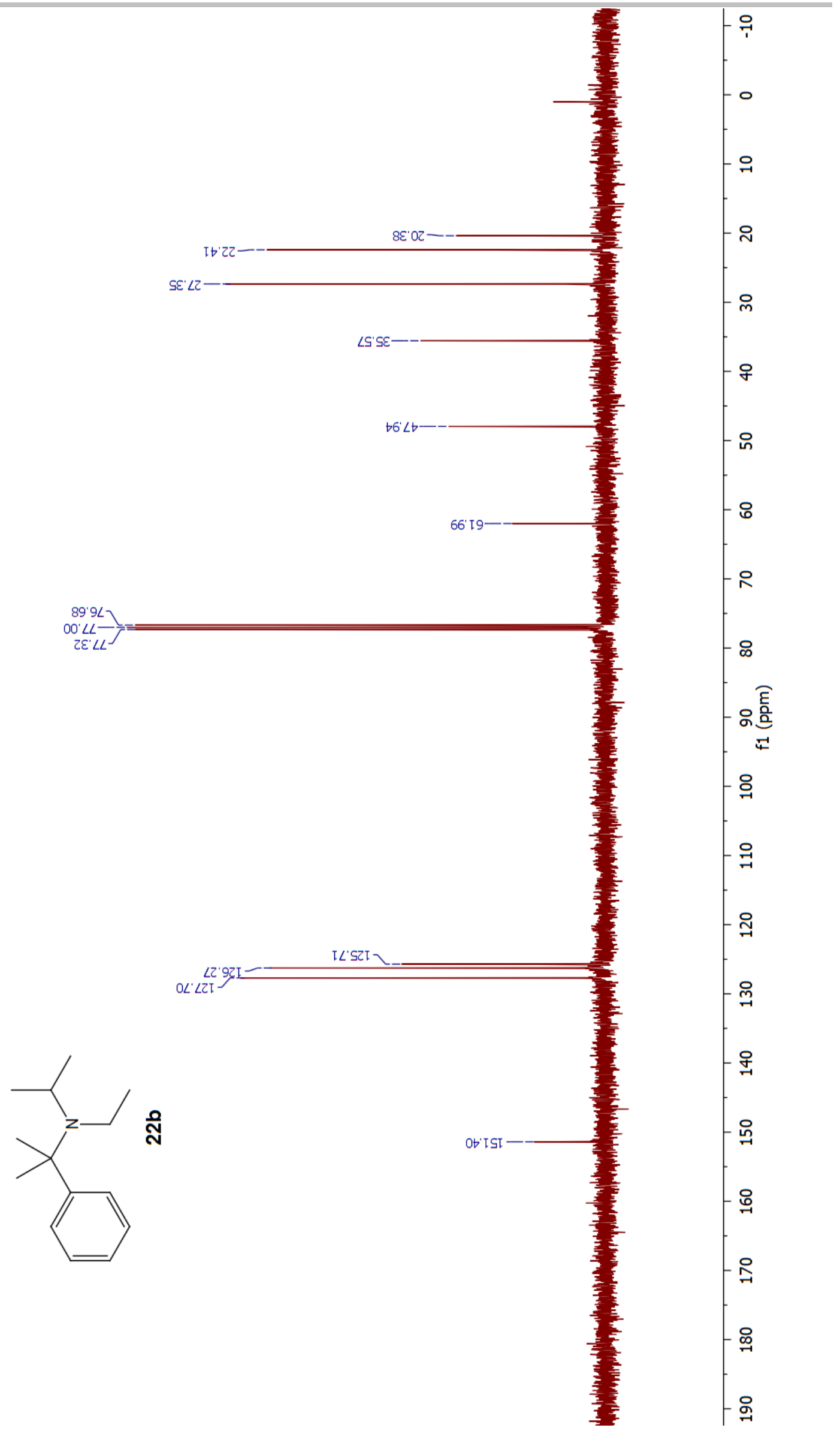

Figure S-26: ${ }^{13} \mathrm{C}\left\{{ }^{1} \mathrm{H}\right\}$ NMR spectrum of $\mathbf{2 2 b}$ in $\mathrm{CDCl}_{3}$. 
(udd) It

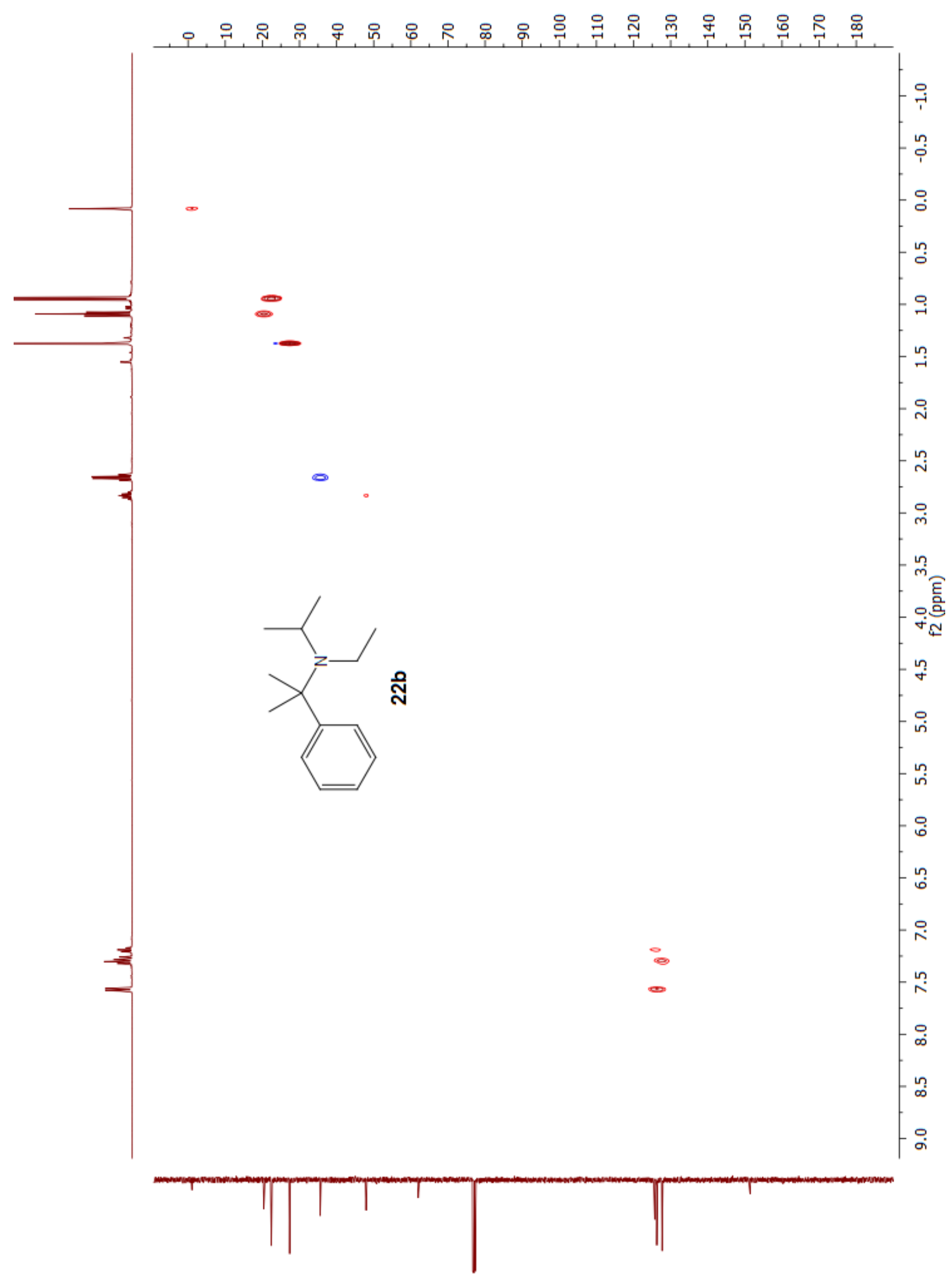

Figure S-27: HSQC 2D NMR spectrum of 22b in $\mathrm{CDCl}_{3}$. 


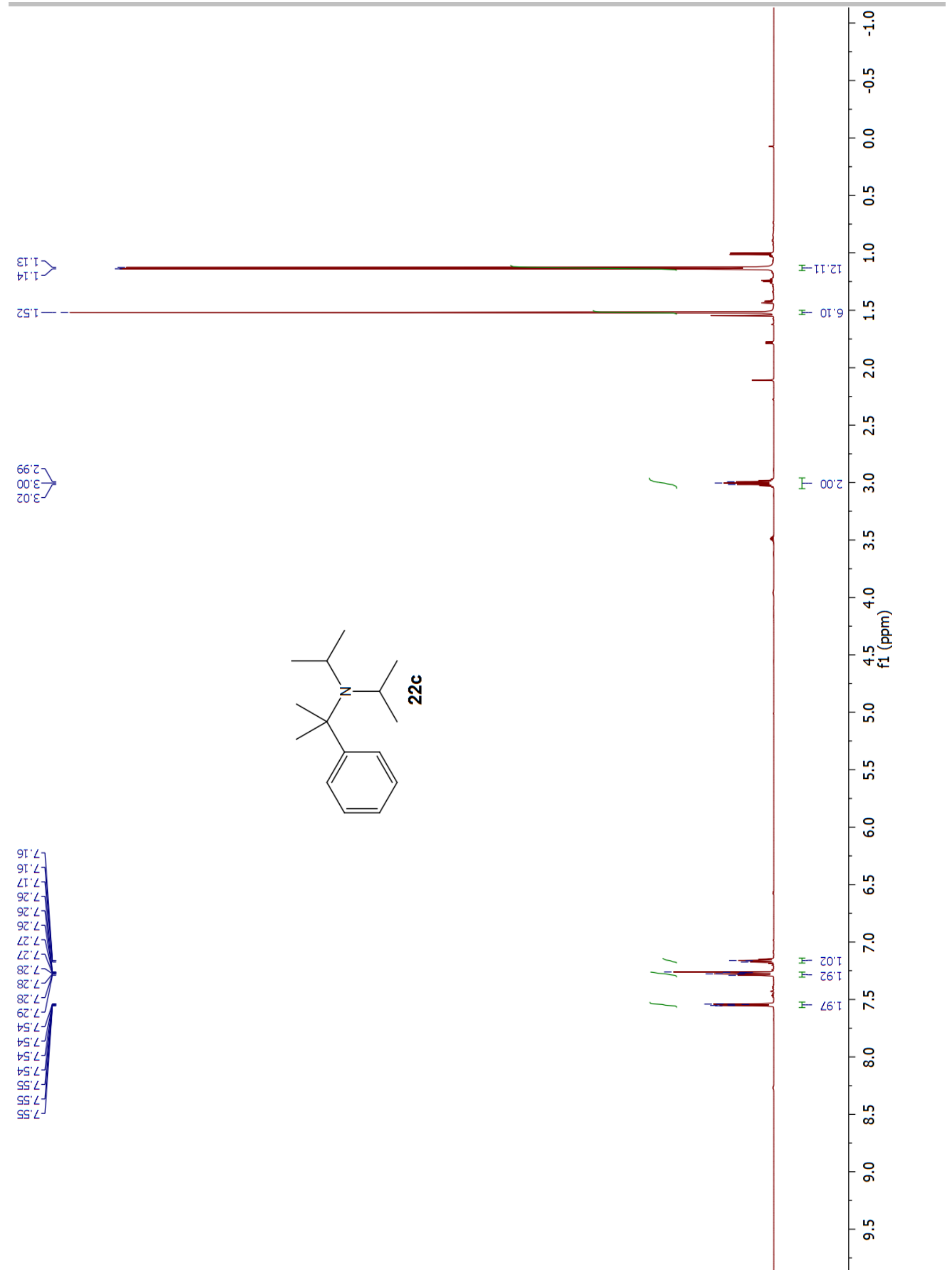

Figure S-28: ${ }^{1} \mathrm{H}$ NMR spectrum of $22 \mathrm{c}$ in $\mathrm{CDCl}_{3}$. 


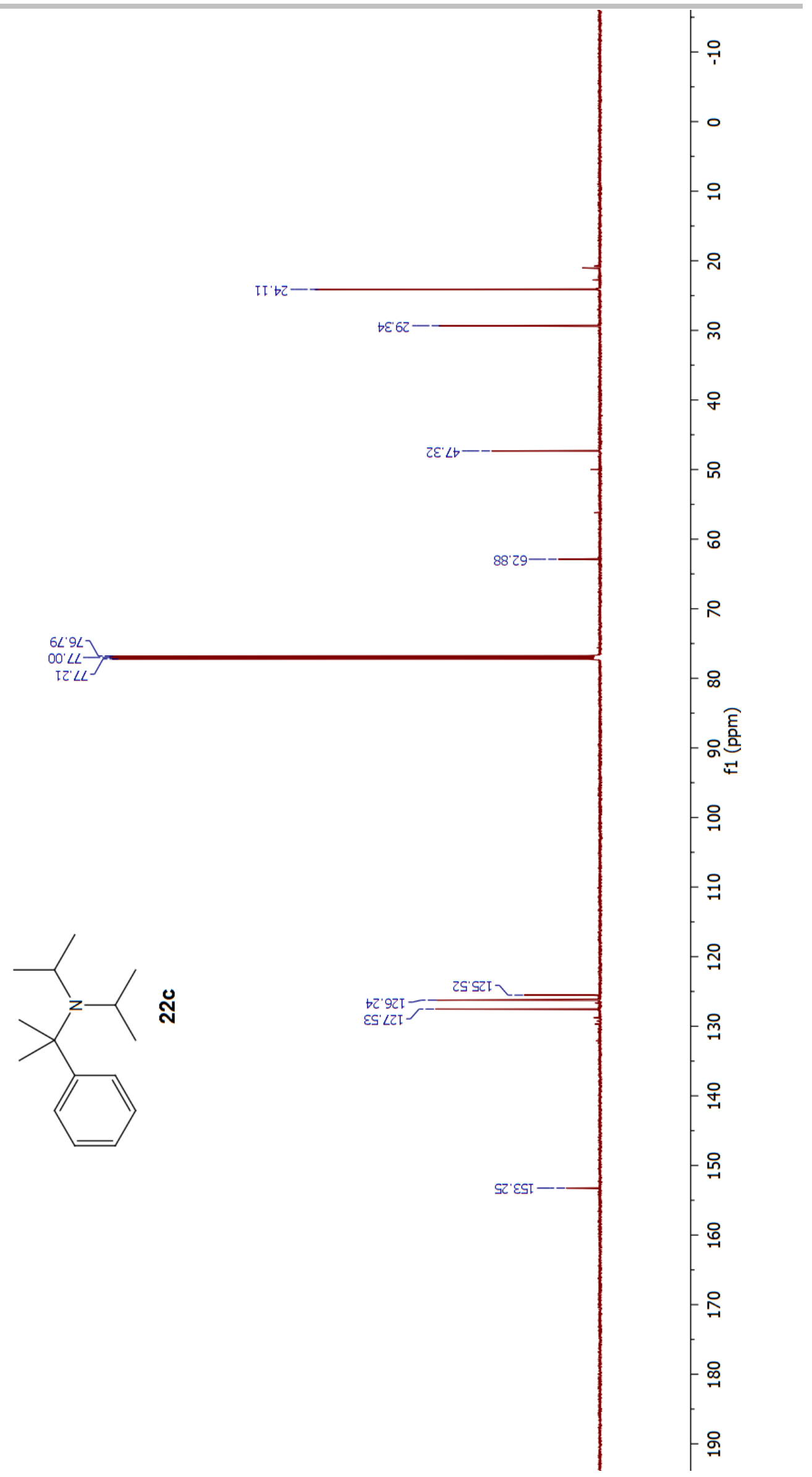

Figure S-29: ${ }^{13} \mathrm{C}\left\{{ }^{1} \mathrm{H}\right\}$ NMR spectrum of $22 \mathrm{c}$ in $\mathrm{CDCl}_{3}$. 


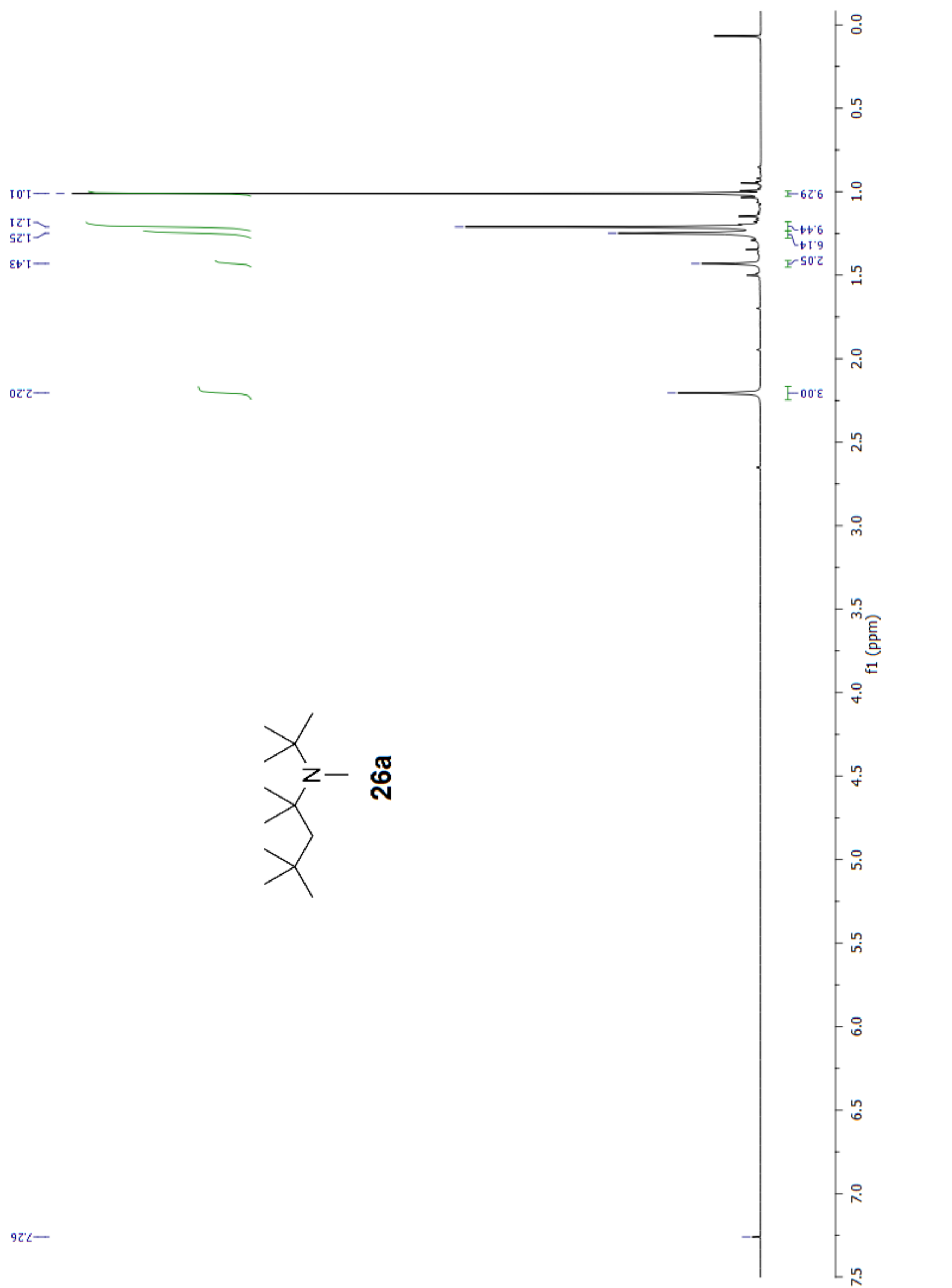

Figure S-30: ${ }^{1} \mathrm{H}$ NMR spectrum of $26 \mathbf{a}$ in $\mathrm{CDCl}_{3}$. 


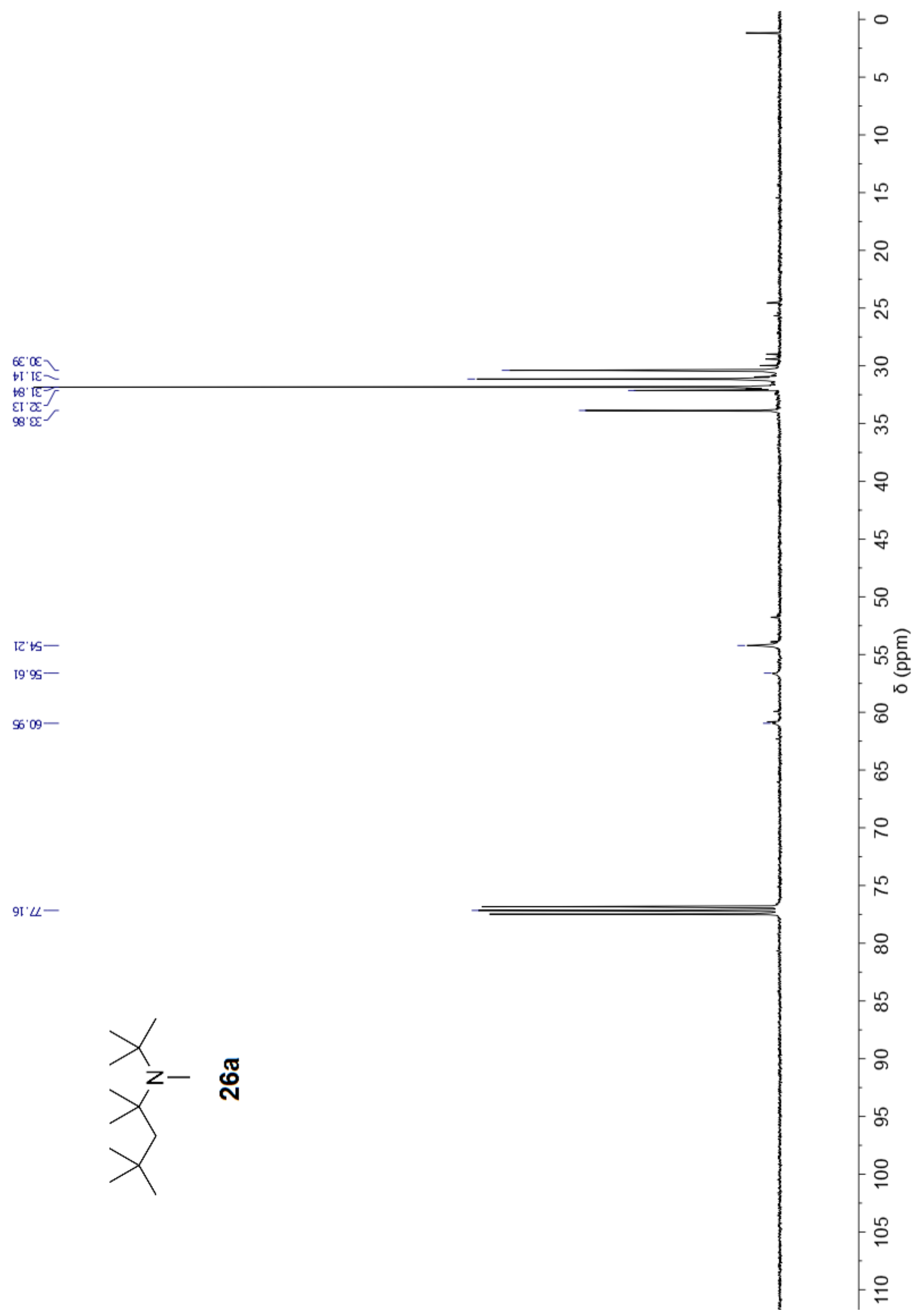

Figure S-31: ${ }^{13} \mathrm{C}\left\{{ }^{1} \mathrm{H}\right\}$ NMR spectrum of $\mathbf{2 6 a}$ in $\mathrm{CDCl}_{3}$. 


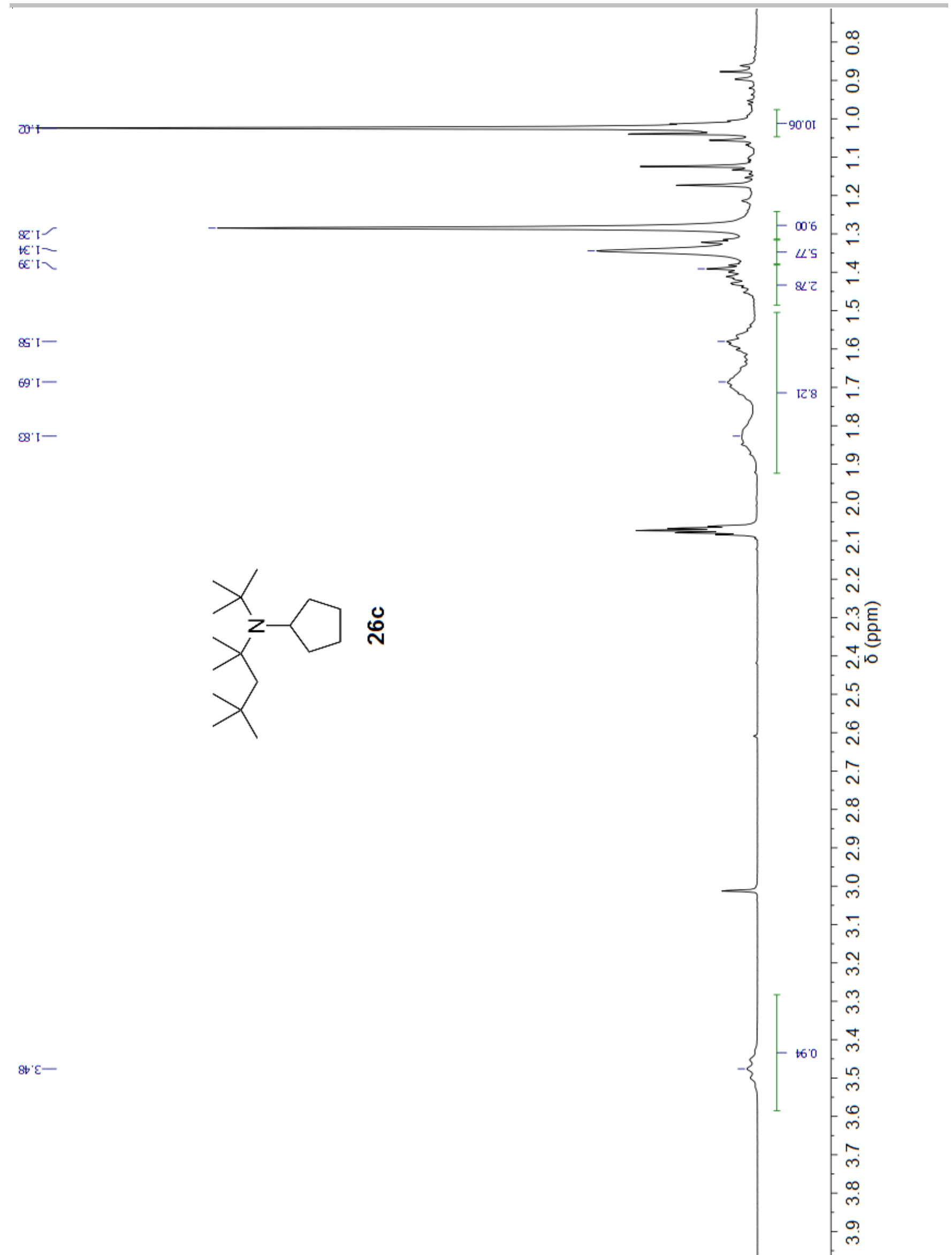

Figure S-32: ${ }^{1} \mathrm{H}$ NMR spectrum of $26 \mathrm{c}$ in $\mathrm{C}_{7} \mathrm{D}_{8}$, which shows a purity of $90 \%$ for this compound; the decay products $\mathrm{N}$-tert-butylcyclopentylamine and $\mathbf{1 0}$ are present with $9 \%$ and $1 \%$, respectively. Broad signals of $\mathbf{2 6 c}$ were induced by molecular dynamics. 


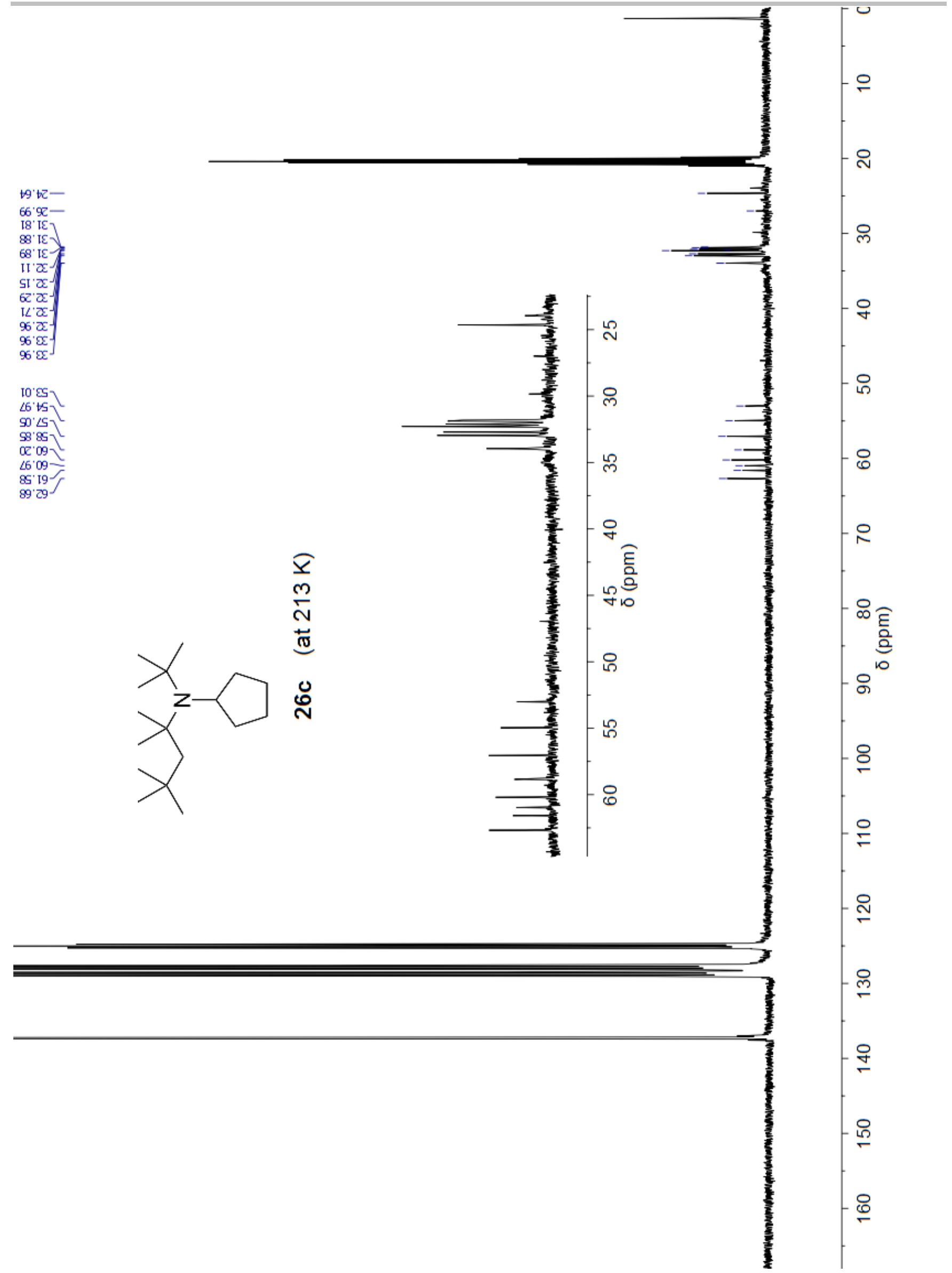

Figure S-33: ${ }^{13} \mathrm{C}\left\{{ }^{1} \mathrm{H}\right\}$ NMR spectrum of $26 \mathrm{c}$ in $\mathrm{C}_{7} \mathrm{D}_{8}$ at $213 \mathrm{~K}$. 


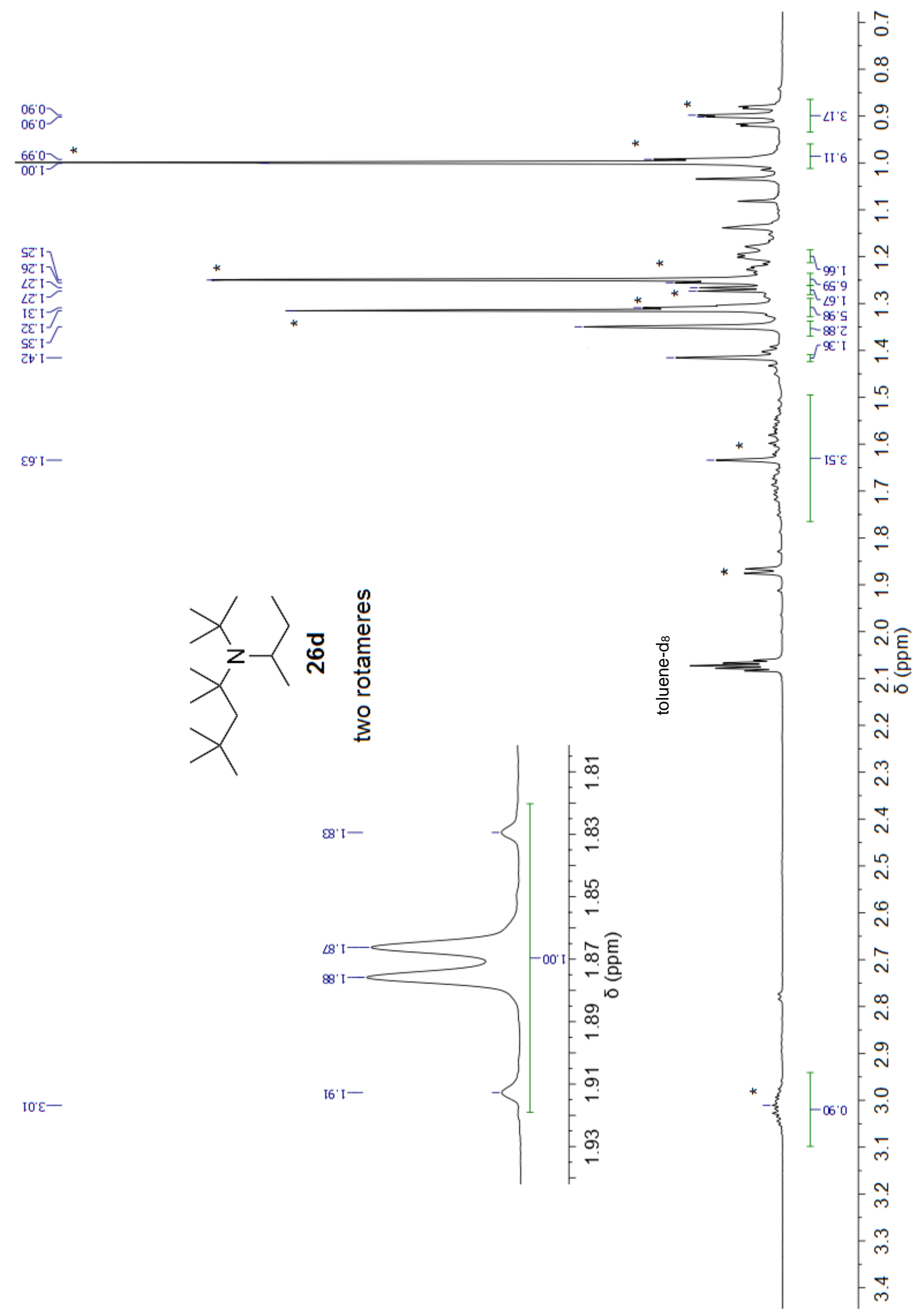

Figure S-34: ${ }^{1} \mathrm{H}$ NMR spectrum of $\mathbf{2 6 d}$ in $\mathrm{C}_{7} \mathrm{D}_{8}$. 
$8 \mathrm{I}^{\prime} \mathrm{I}$

S. I

政政

天'坛了

If. I

$29 \cdot 1-$

เ8'

$08^{\prime} \mathrm{C}$

$08^{\prime} z-$
$68^{\prime} z-$

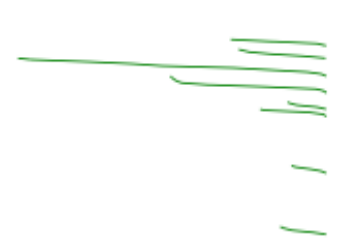

$\stackrel{\Xi}{\circ}$

Figure S-35: ${ }^{1} \mathrm{H}$ NMR spectrum of $26 e$ in $\mathrm{C}_{7} \mathrm{D}_{8}$.

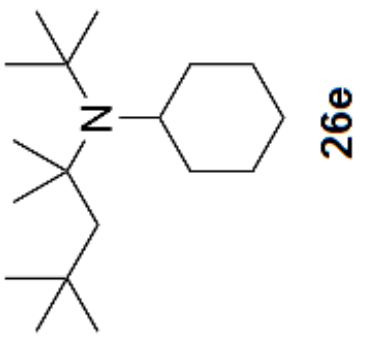

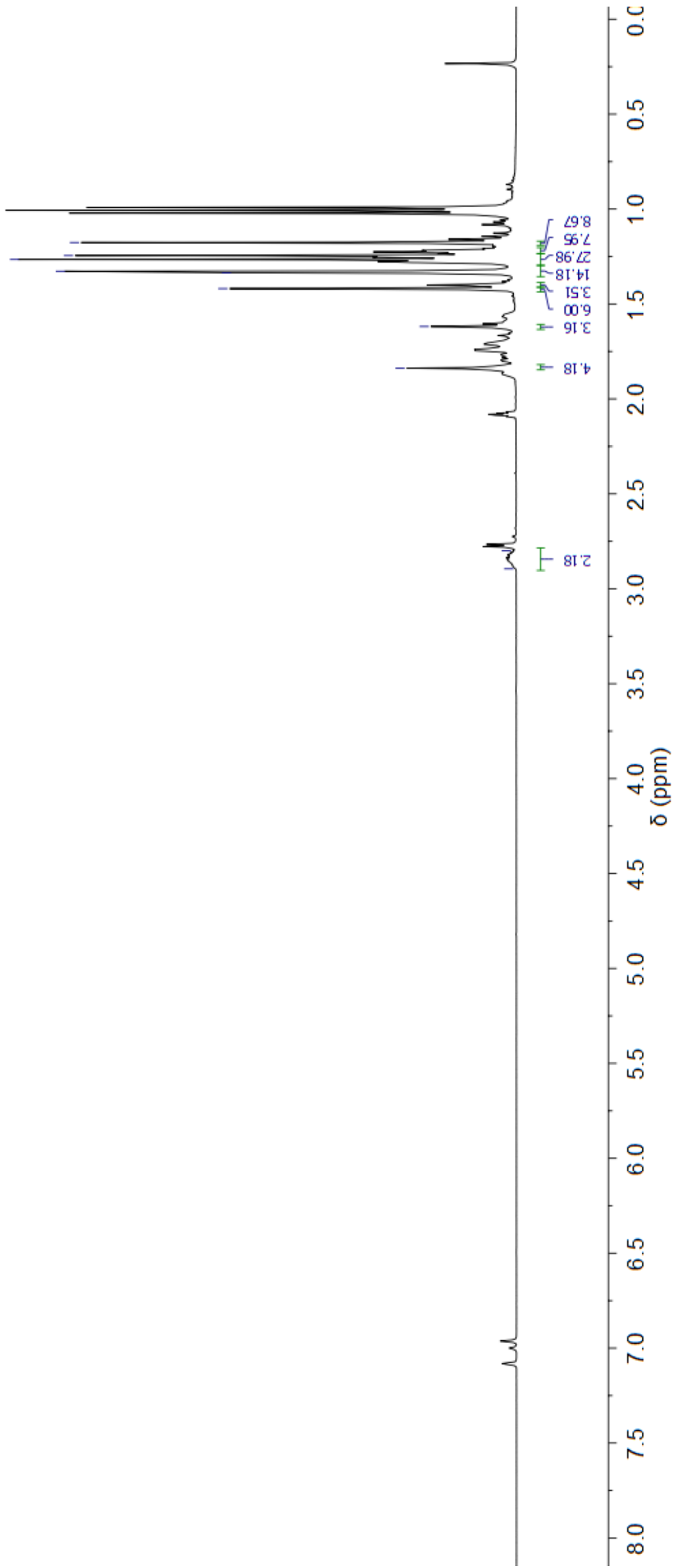

$\infty_{\infty}^{\infty}$ 


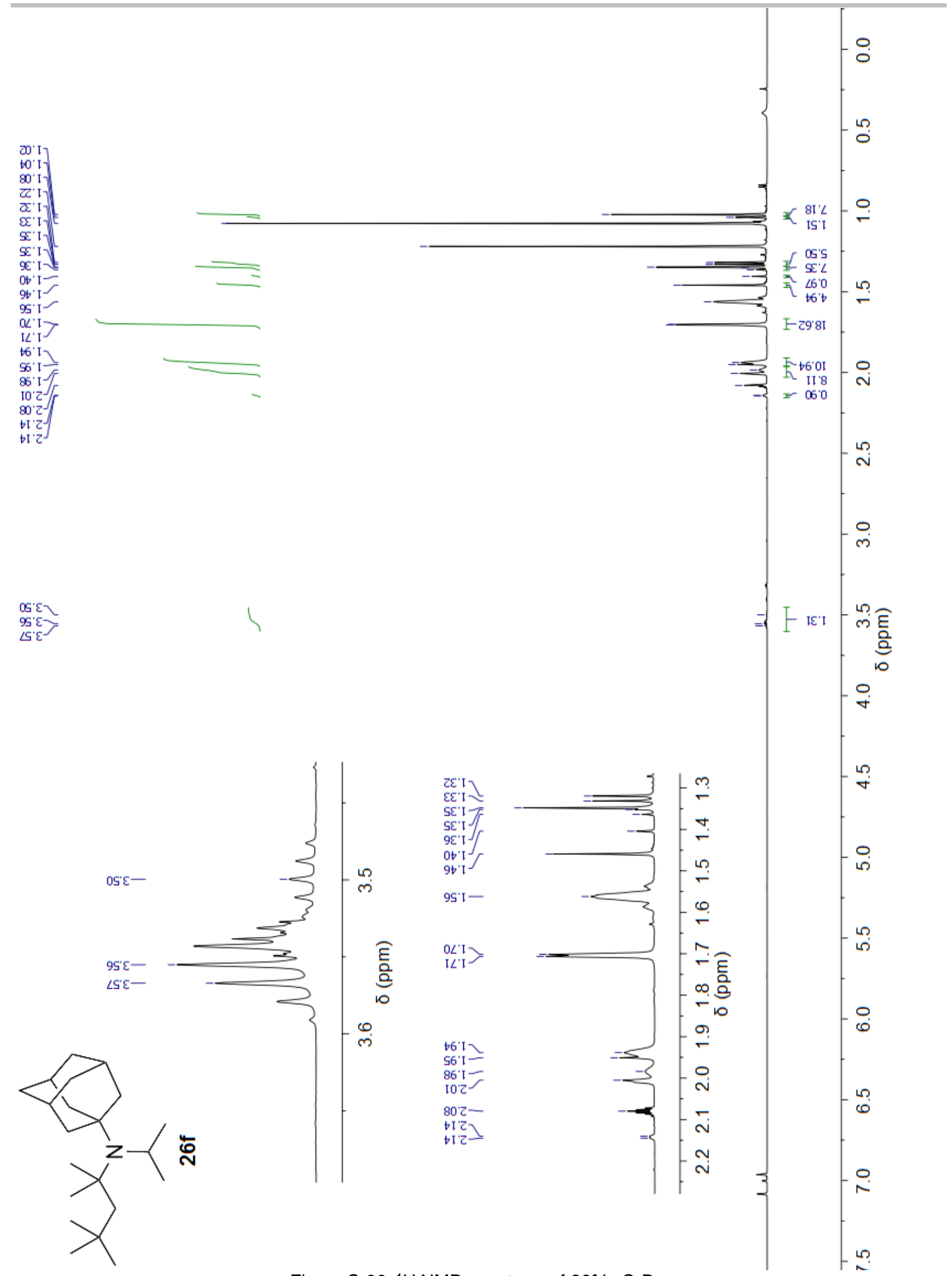

Figure S-36: ${ }^{1} \mathrm{H}$ NMR spectrum of $\mathbf{2 6 f}$ in $\mathrm{C}_{7} \mathrm{D}_{8}$. 


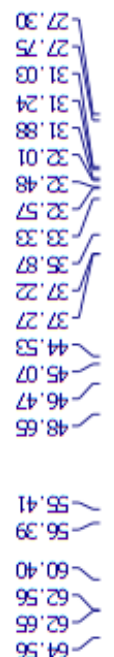

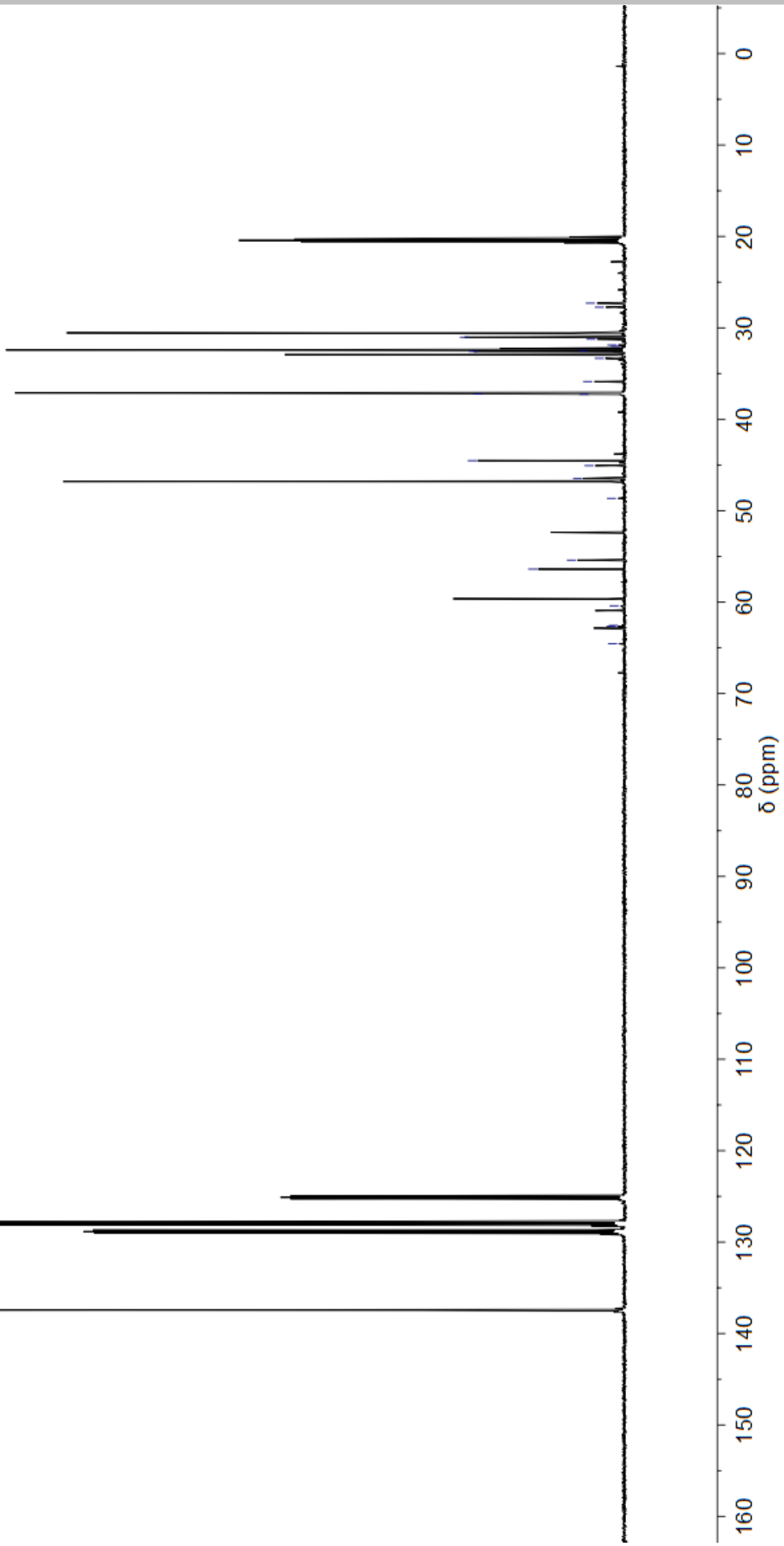

Figure S-37: ${ }^{13} \mathrm{C}\left\{{ }^{1} \mathrm{H}\right\}$ NMR spectrum of $26 f$ in $\mathrm{C}_{7} \mathrm{D}_{8}$. 
(wdd) $Q$

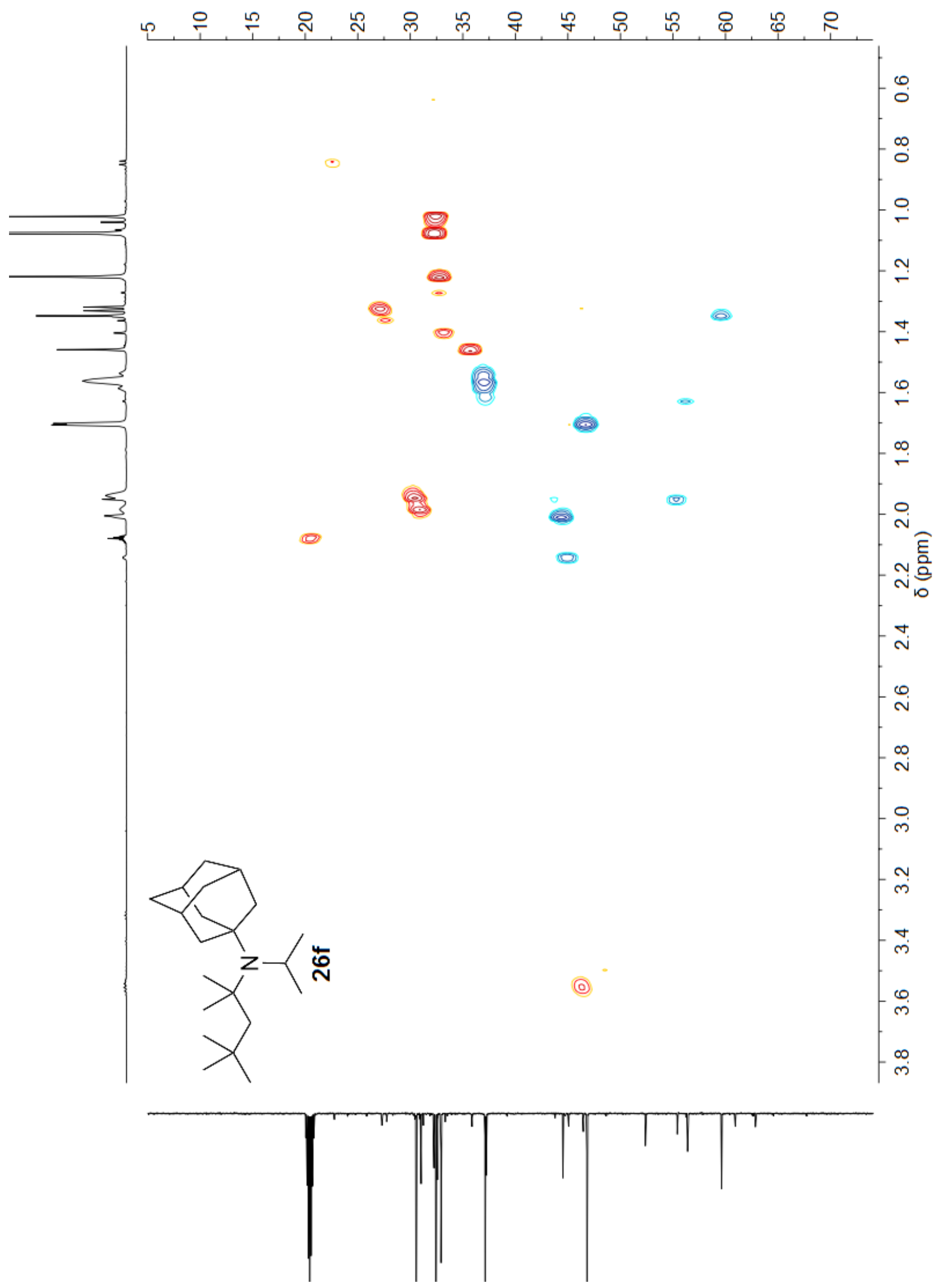

Figure S-38: HSQC 2D NMR spectrum of $26 f$ in $C_{7} D_{8}$. 


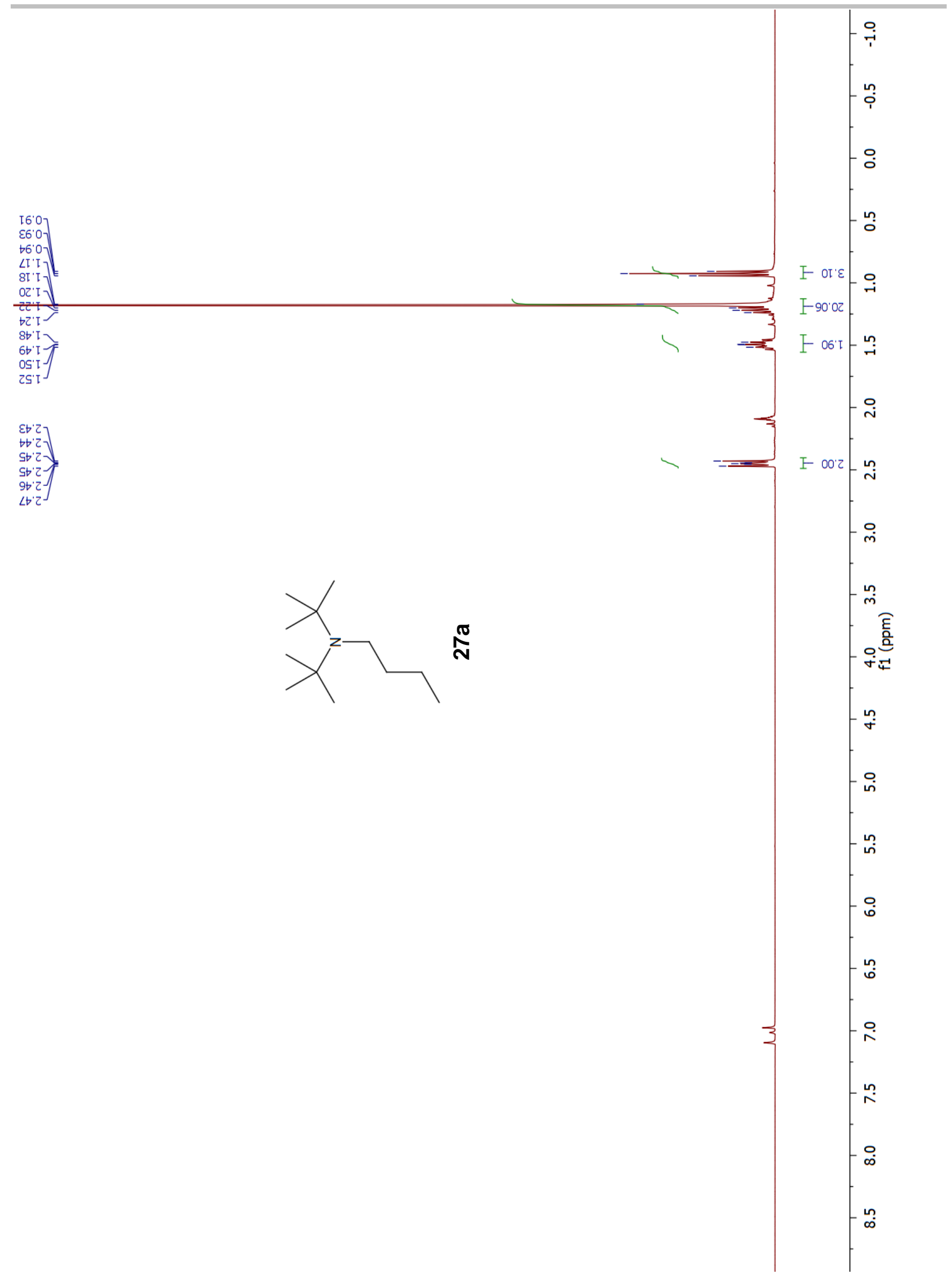

Figure S-39: ${ }^{1} \mathrm{H}$ NMR spectrum of $27 \mathrm{a}$ in $\mathrm{C}_{7} \mathrm{D}_{8}$. 


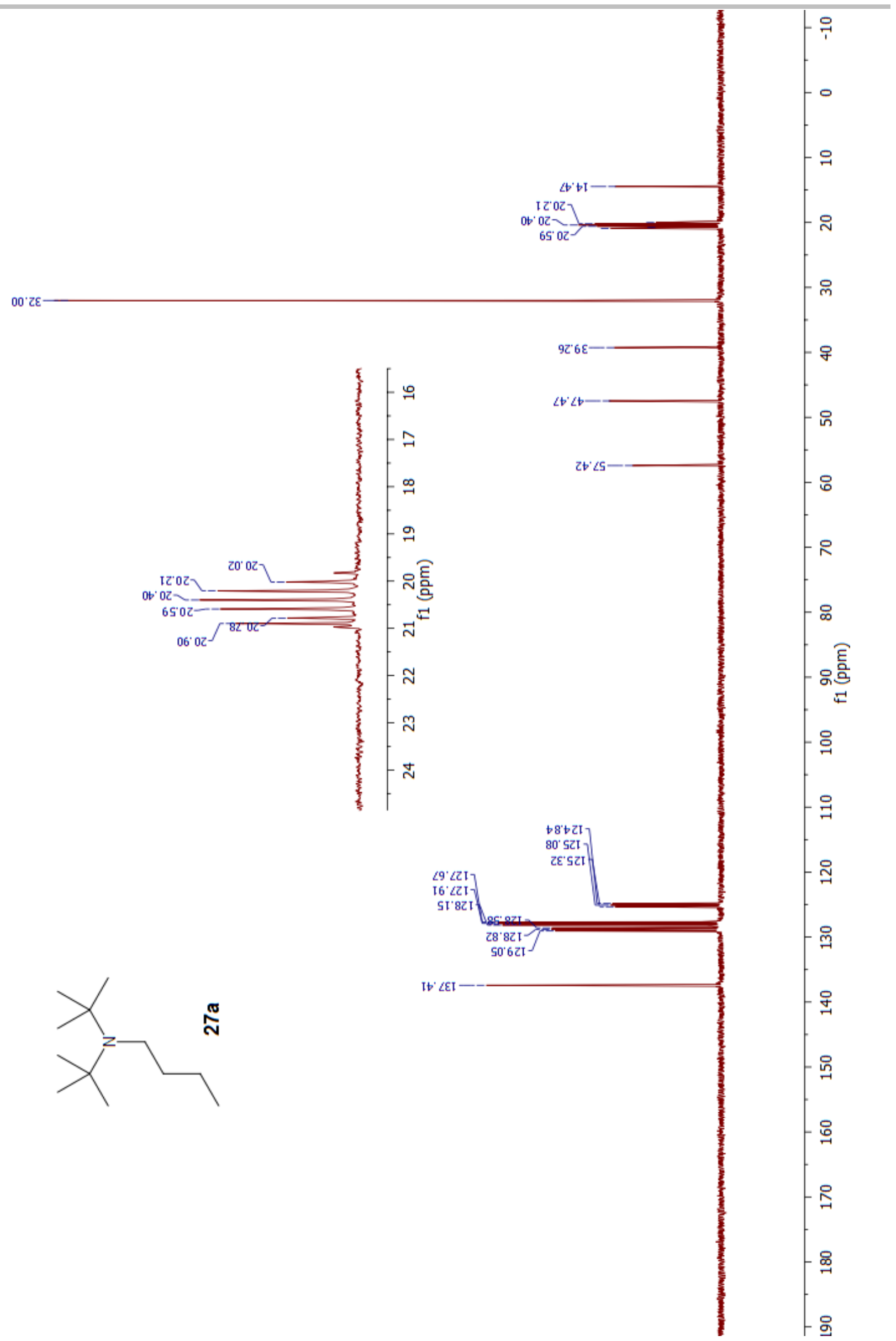

Figure S-40: ${ }^{13} \mathrm{C}\left\{{ }^{1} \mathrm{H}\right\}$ NMR spectrum of $27 \mathrm{a}$ in $\mathrm{C}_{7} \mathrm{D}_{8}$. 


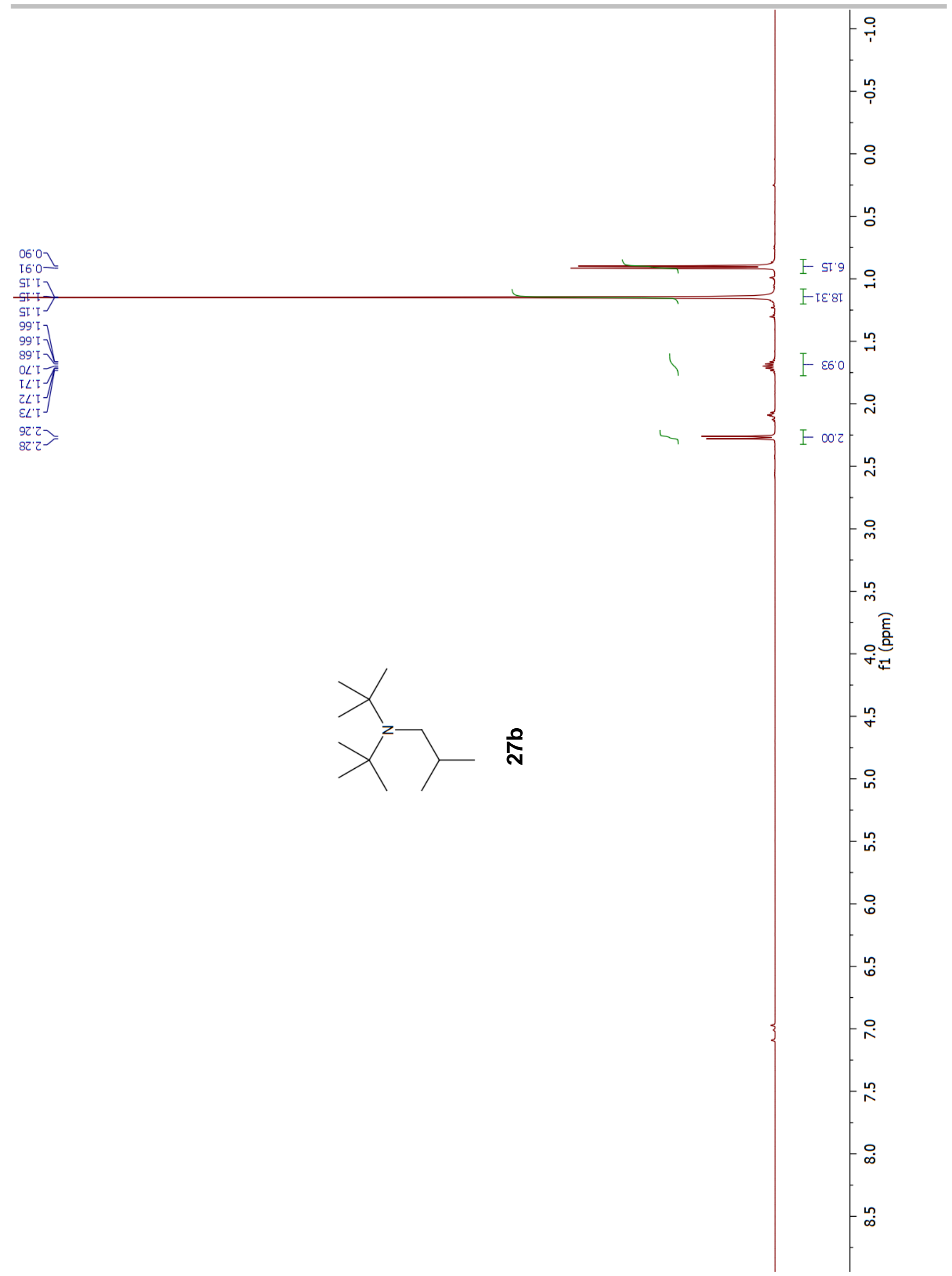

Figure S-41: ${ }^{1} \mathrm{H}$ NMR spectrum of $\mathbf{2 7} \mathbf{b}$ in $\mathrm{C}_{7} \mathrm{D}_{8}$. 


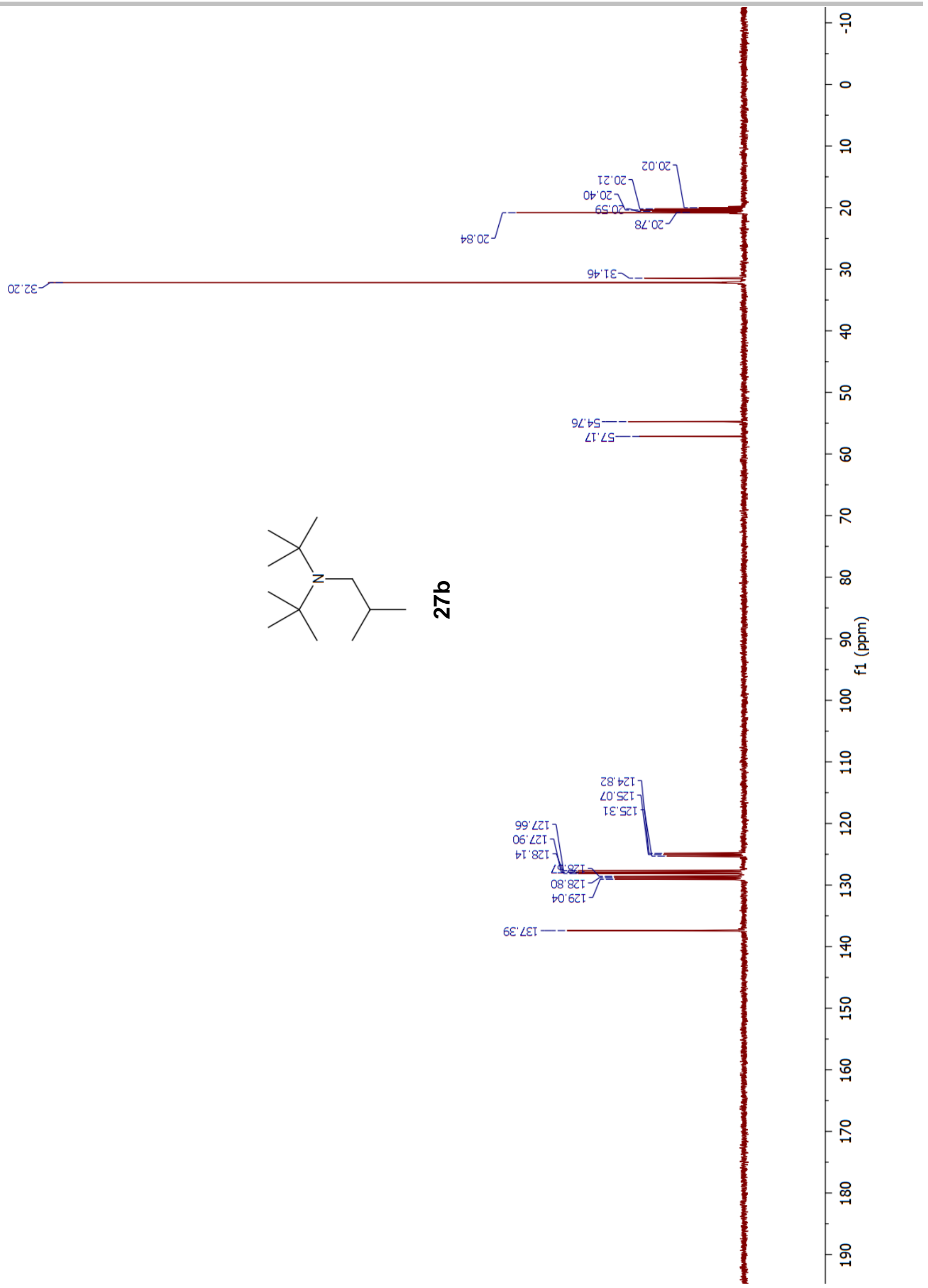

Figure S-42: ${ }^{13} \mathrm{C}\left\{{ }^{1} \mathrm{H}\right\}$ NMR spectrum of $\mathbf{2 7} \mathbf{b}$ in $\mathrm{C}_{7} \mathrm{D}_{8}$. 


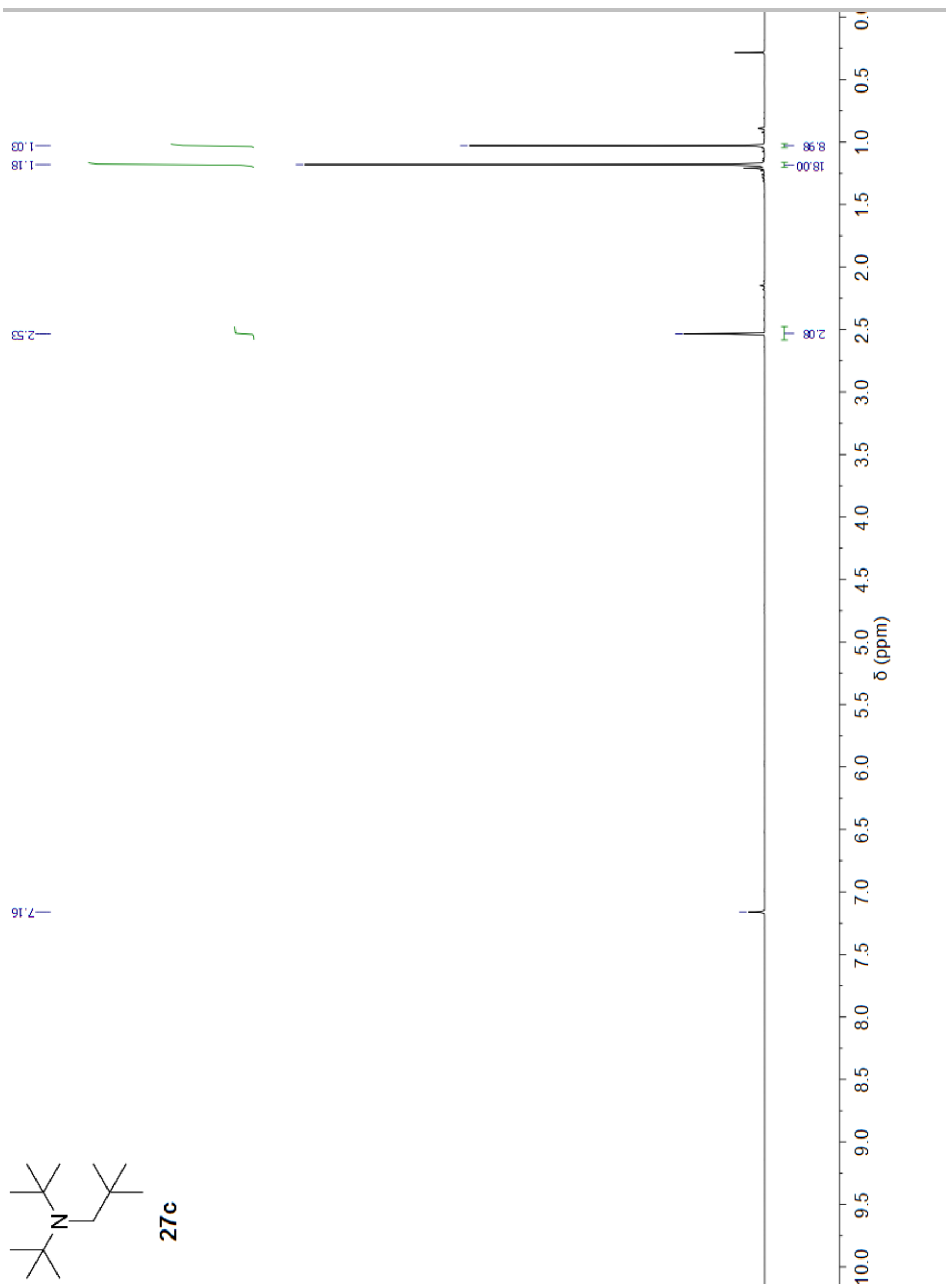

Figure S-43: ${ }^{1} \mathrm{H}$ NMR spectrum of $27 \mathrm{c}$ in $\mathrm{C}_{6} \mathrm{D}_{6}$. 


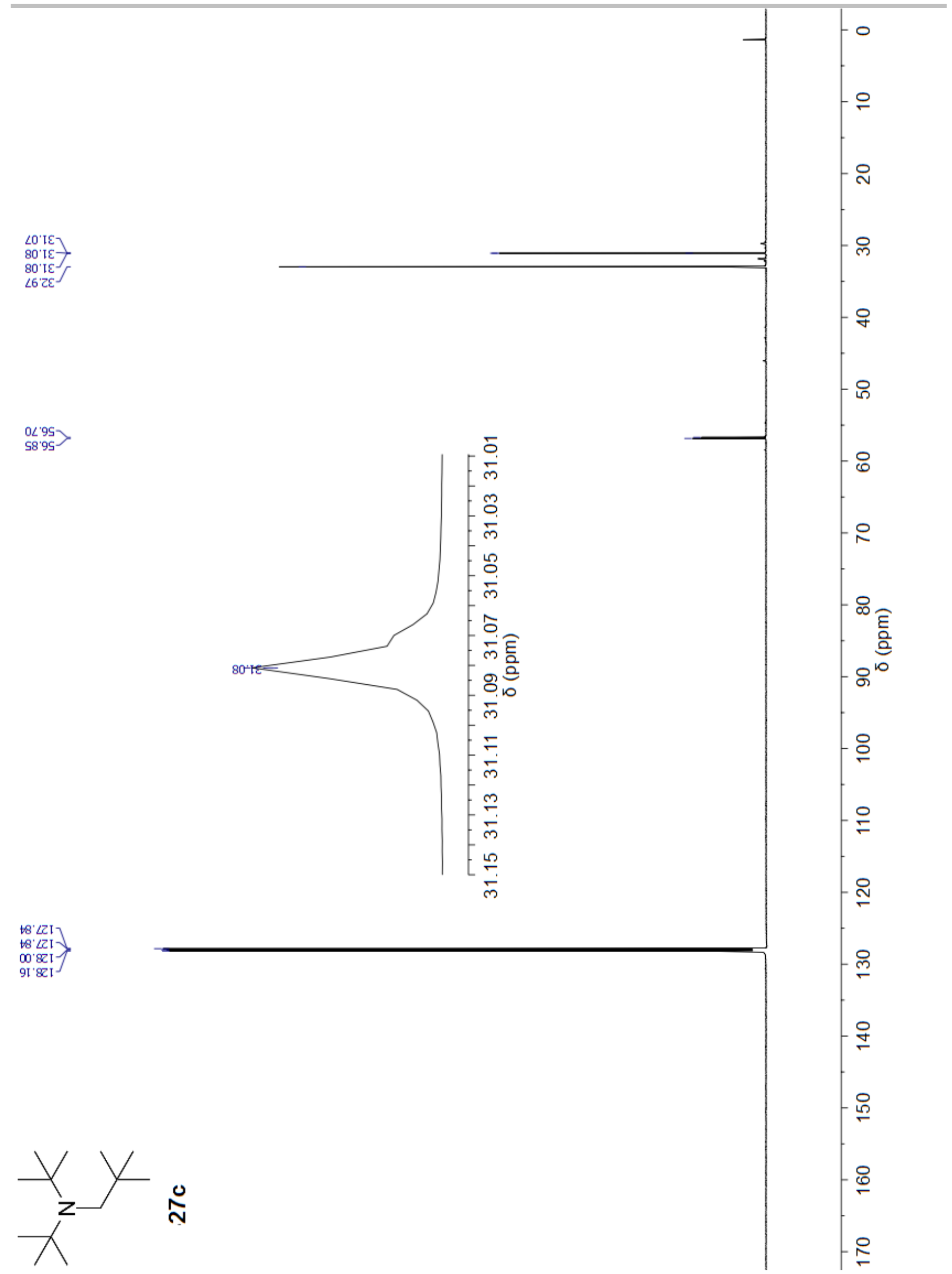

Figure S-44: ${ }^{13} \mathrm{C}$ NMR spectrum of $27 \mathrm{c}$ in $\mathrm{C}_{6} \mathrm{D}_{6}$. 


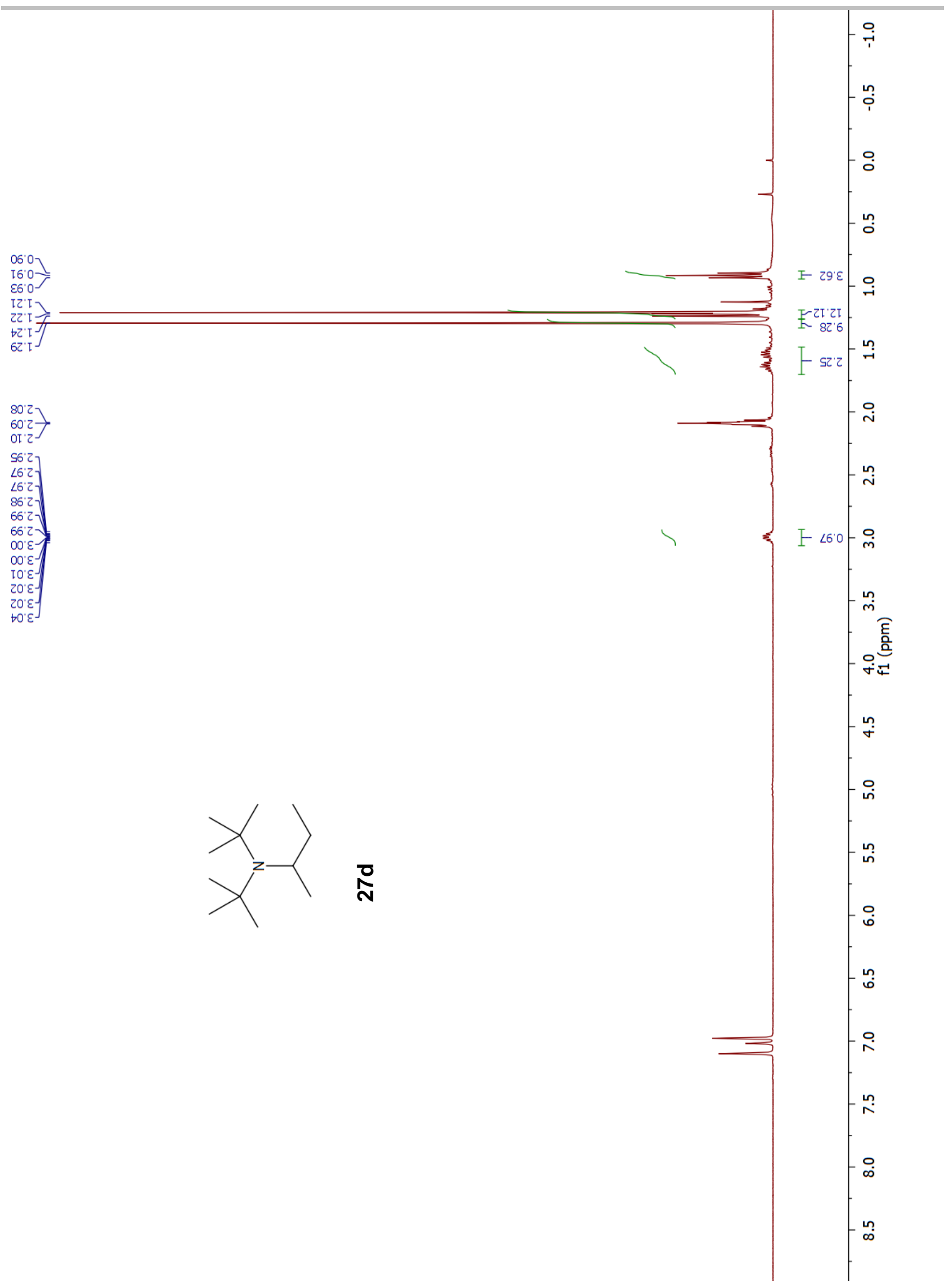

Figure S-45: ${ }^{1} \mathrm{H}$ NMR spectrum of $\mathbf{2 7 d}$ in $\mathrm{C}_{7} \mathrm{D}_{8}$. 


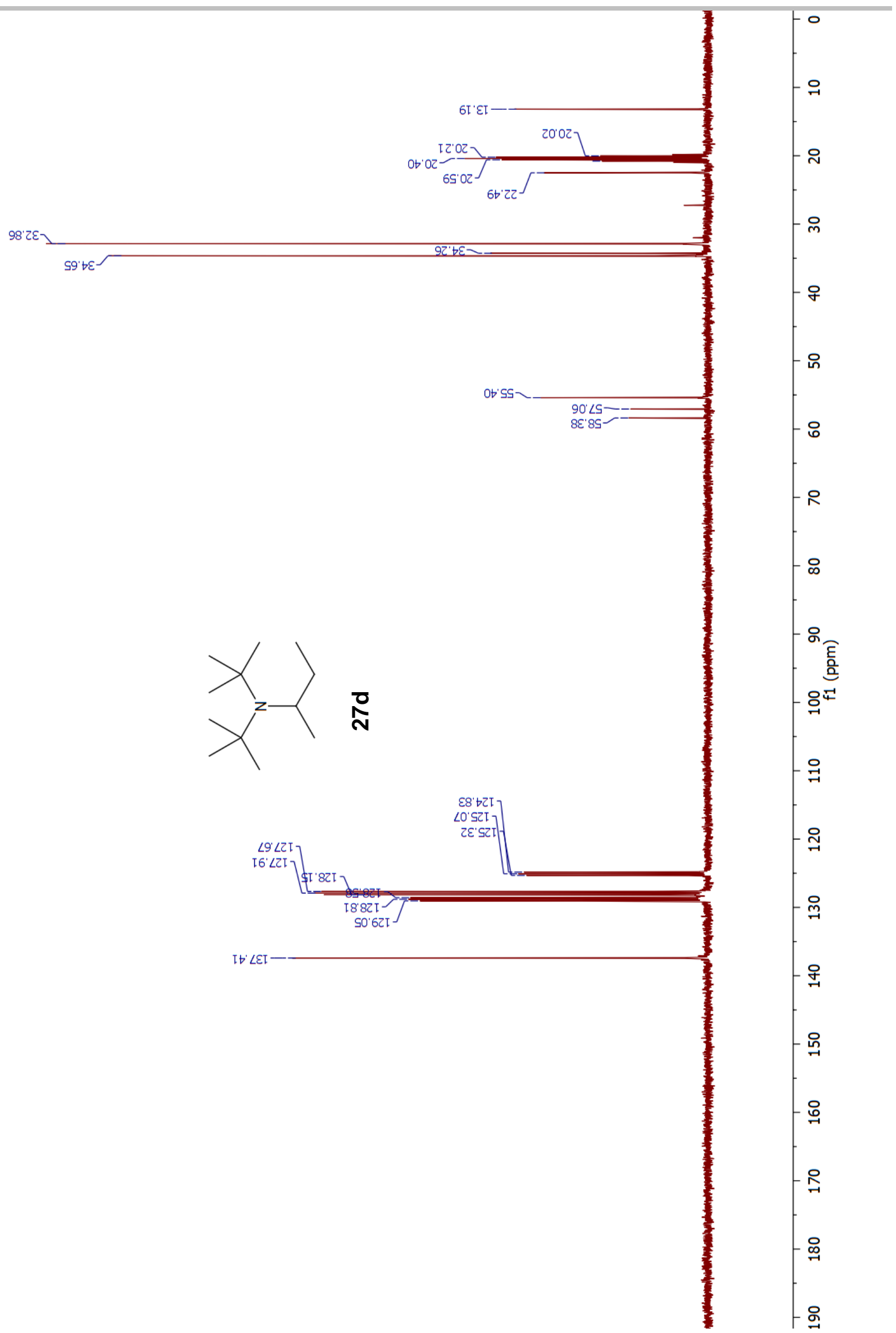

Figure $\mathrm{S}-46:{ }^{13} \mathrm{C}\left\{{ }^{1} \mathrm{H}\right\}$ NMR spectrum of $\mathbf{2 7 d}$ in $\mathrm{C}_{7} \mathrm{D}_{8}$. 


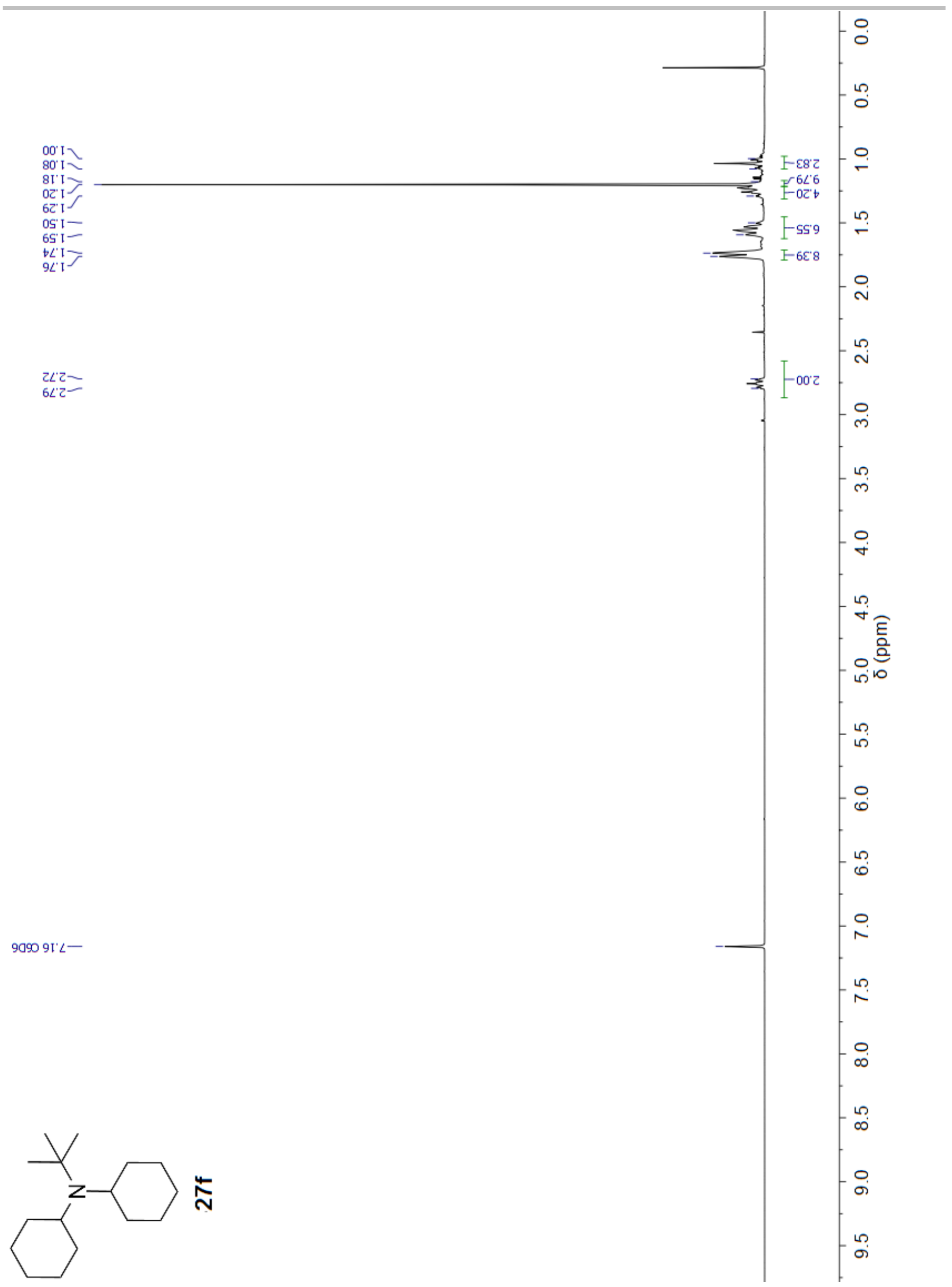

Figure S-47: ${ }^{1} \mathrm{H}$ NMR spectrum of $27 \mathrm{f}$ in $\mathrm{C}_{6} \mathrm{D}_{6}$. 


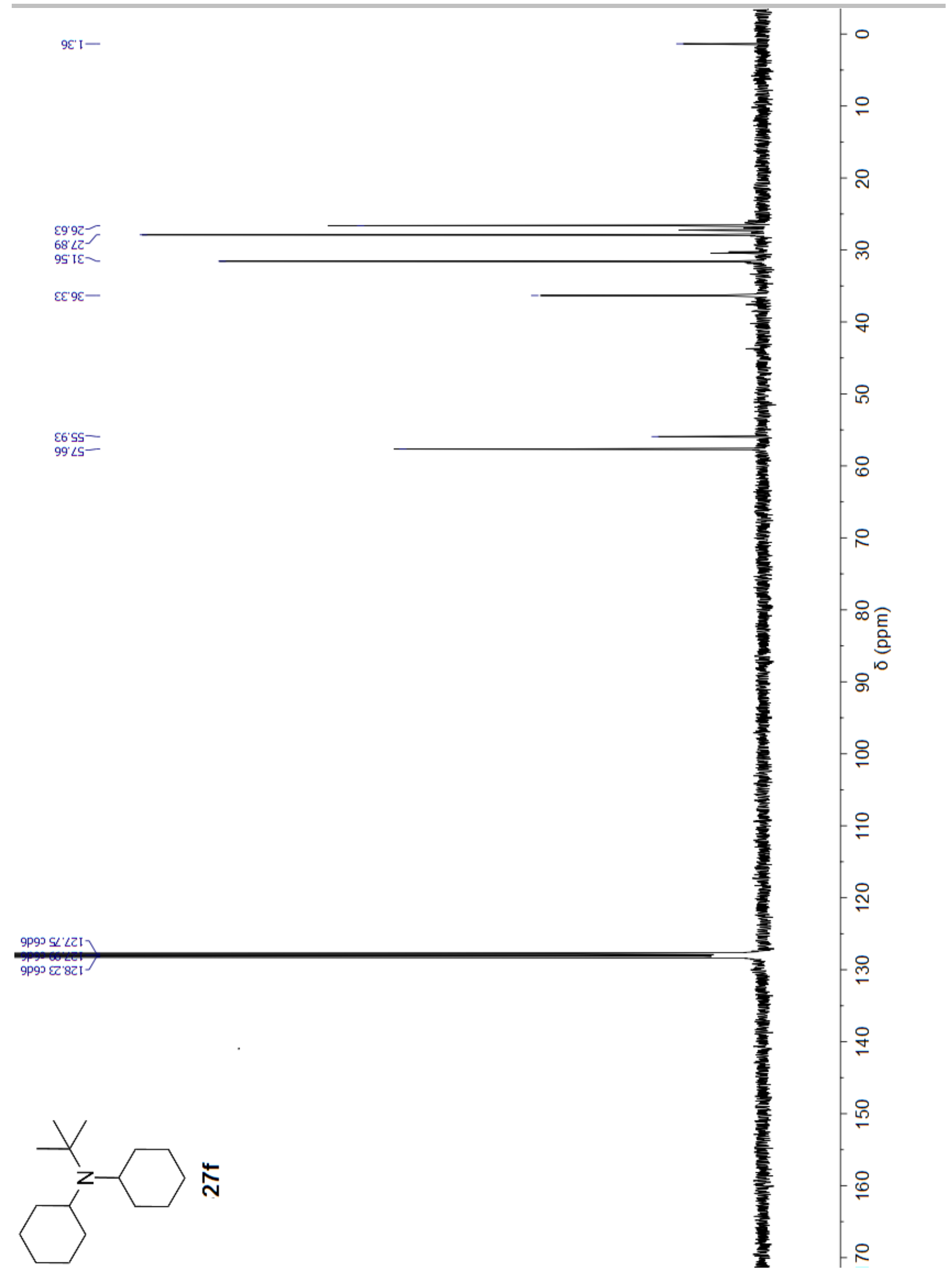

Figure S-48: ${ }^{13} \mathrm{C}\left\{{ }^{1} \mathrm{H}\right\}$ NMR spectrum of $27 \mathrm{f}$ in $\mathrm{C}_{6} \mathrm{D}_{6}$. 
$66^{\circ} 0$
96.0
86.0
$22^{\circ}$
1

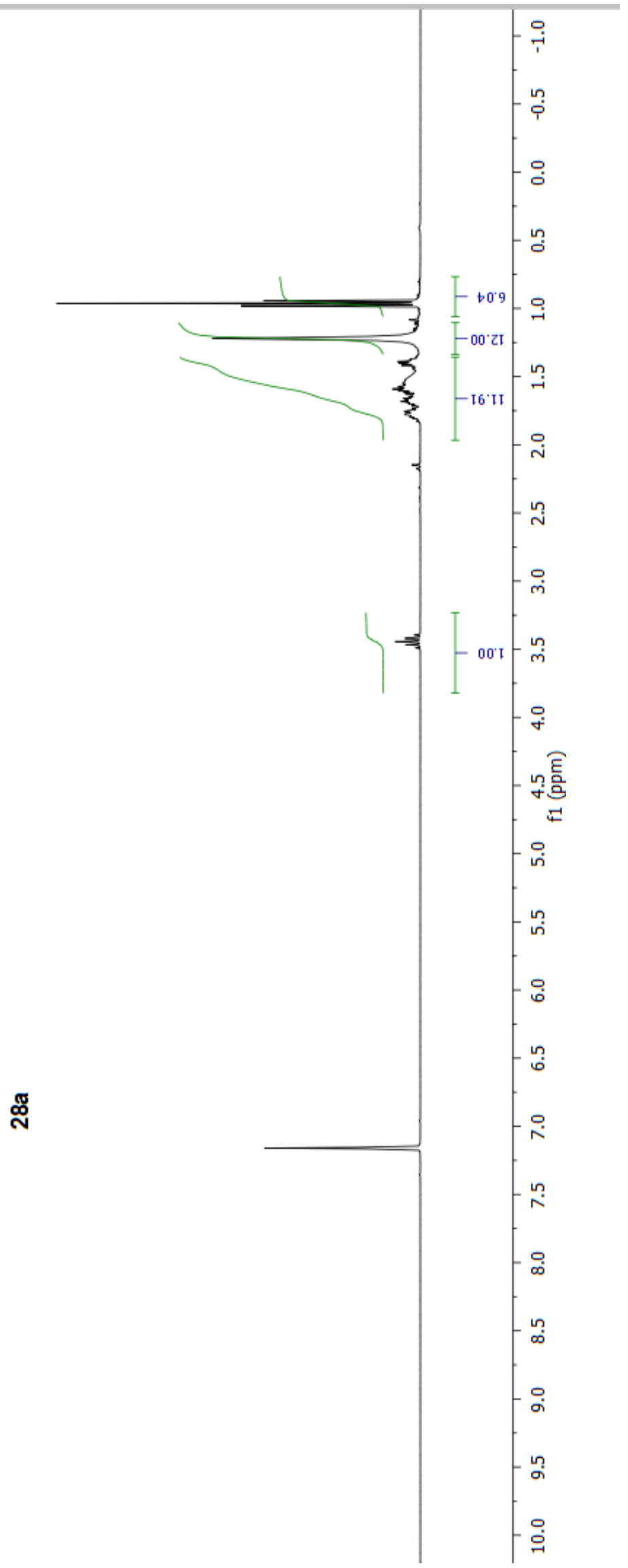

Figure S-49: ${ }^{1} \mathrm{H}$ NMR spectrum of $\mathbf{2 8 a}$ in $\mathrm{C}_{6} \mathrm{D}_{6}$. 


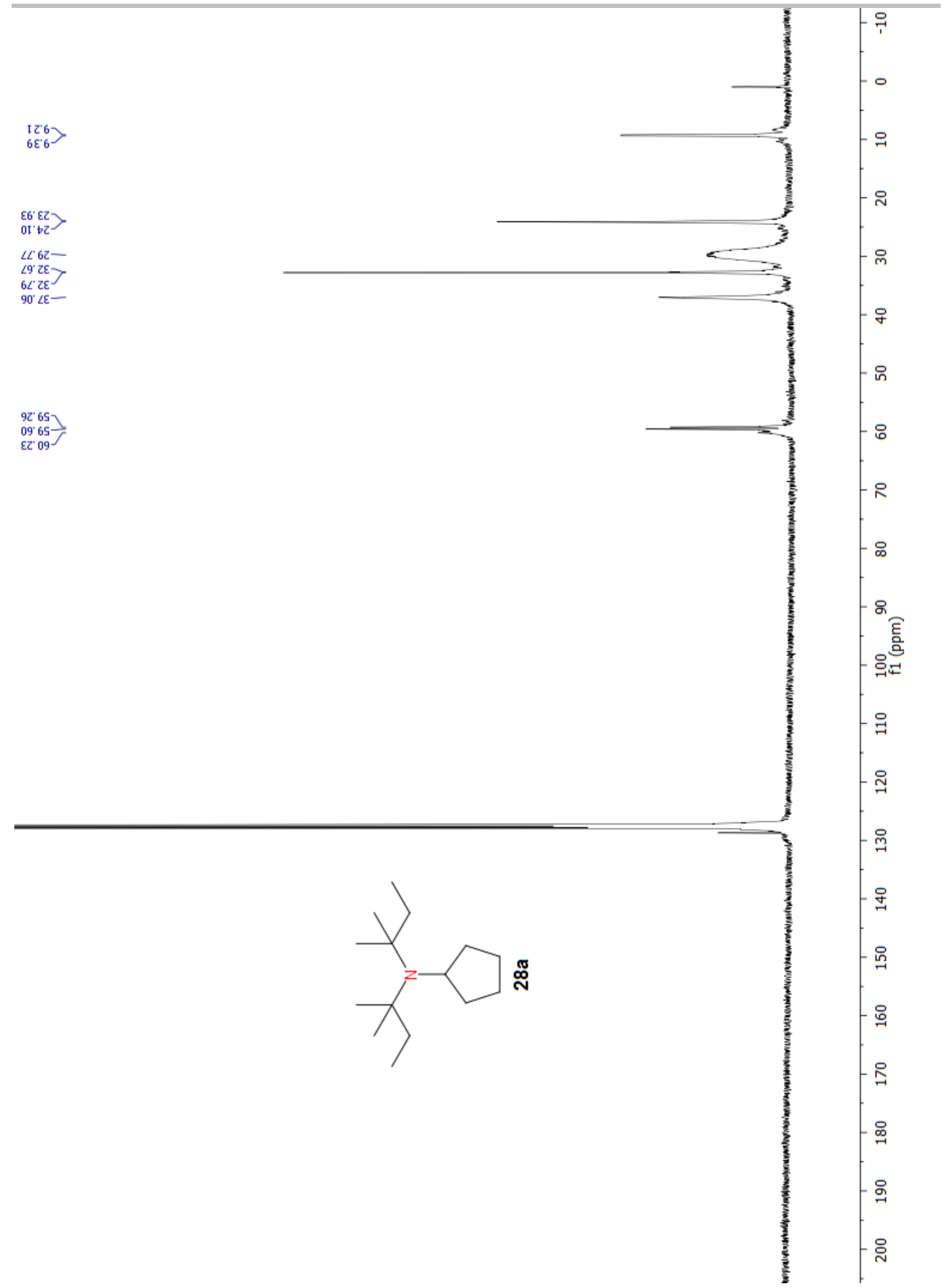

Figure S-50: ${ }^{13} \mathrm{C}\left\{{ }^{1} \mathrm{H}\right\}$ NMR spectrum of $28 \mathrm{a}$ in $\mathrm{C}_{6} \mathrm{D}_{6}$. 


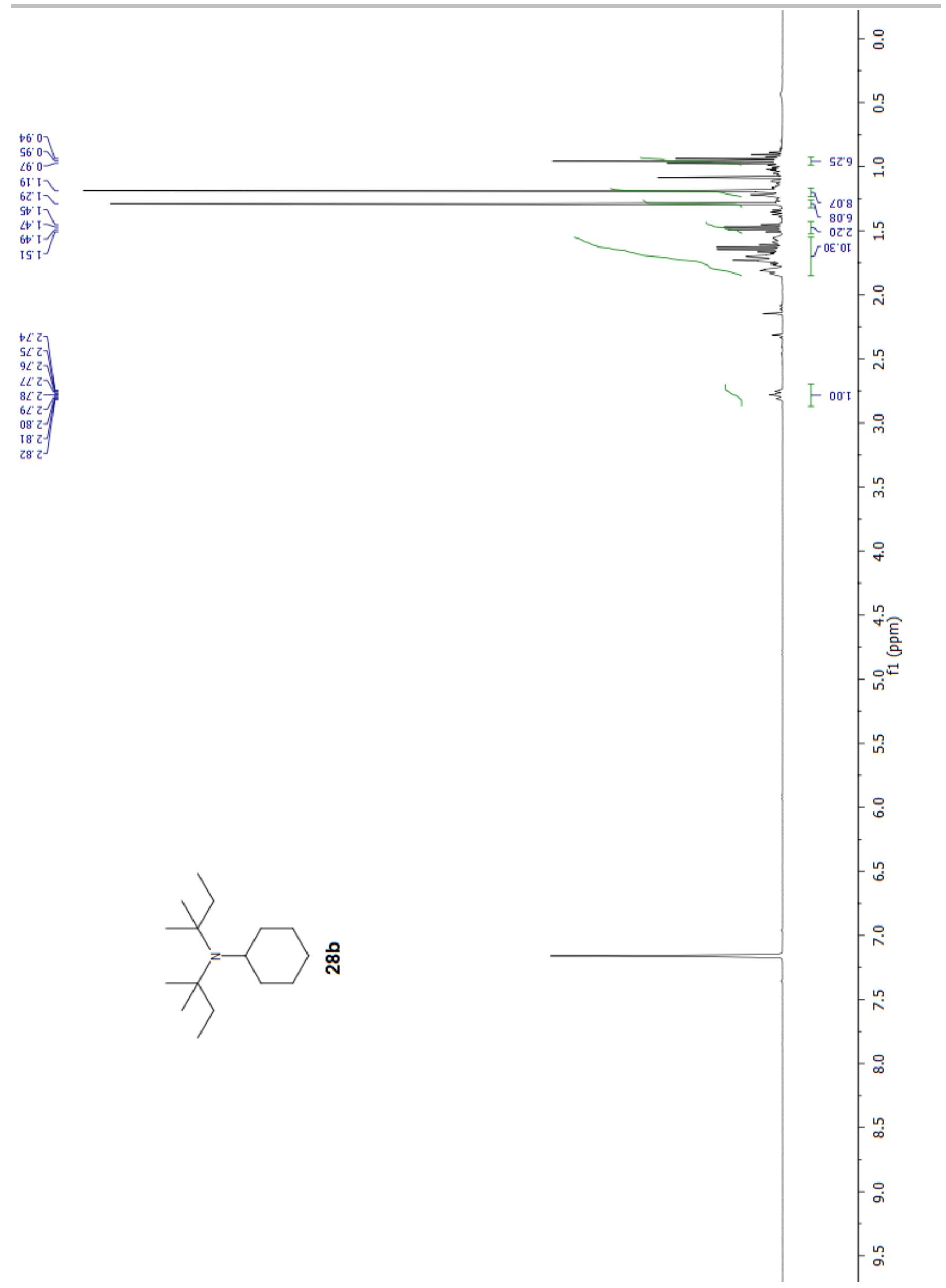

Figure S-51: ${ }^{1} \mathrm{H}$ NMR spectrum of $\mathbf{2 8 b}$ in $\mathrm{C}_{6} \mathrm{D}_{6}$. 


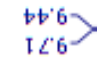

s9. 92

$9 b^{\prime} 8 z$
$08.8 z$

โ9' $1 \varepsilon^{\circ}$

08.

So $\angle \varepsilon-\gamma$

Et $\angle \varepsilon$

St. 65)

85. 65
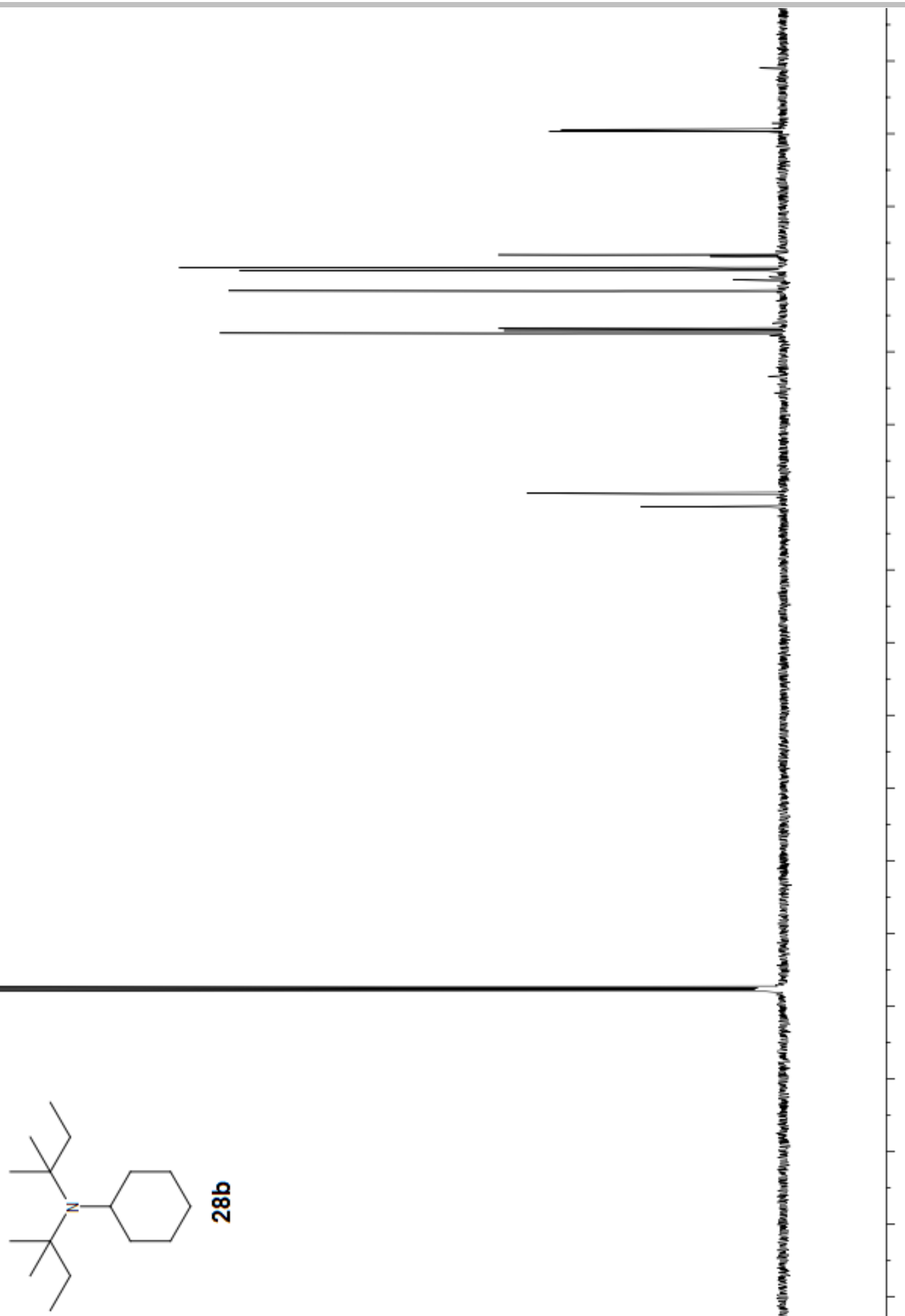

Figure S-52: ${ }^{13} \mathrm{C}\left\{{ }^{1} \mathrm{H}\right\}$ NMR spectrum of $\mathbf{2 8 b}$ in $\mathrm{C}_{6} \mathrm{D}_{6}$. 


\section{SUPPORTING INFORMATION}

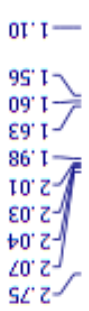

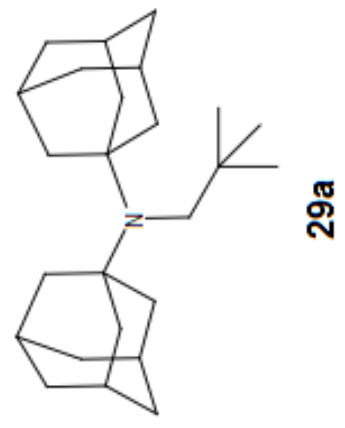

ฉั

Figure S-53: ${ }^{1} \mathrm{H}$ NMR spectrum of $29 a$ in $\mathrm{C}_{6} \mathrm{D}_{6}$. 

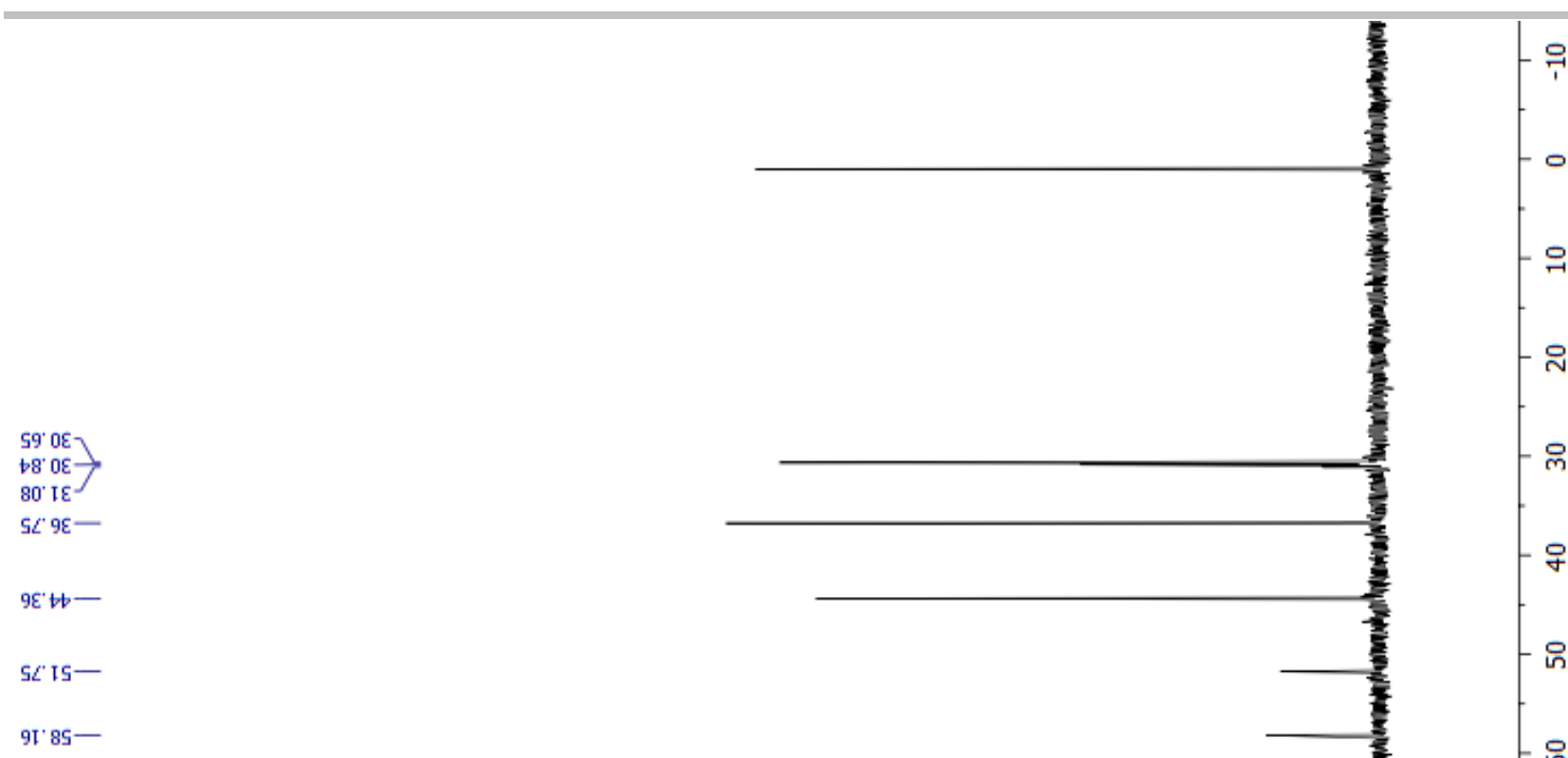

9I' 85

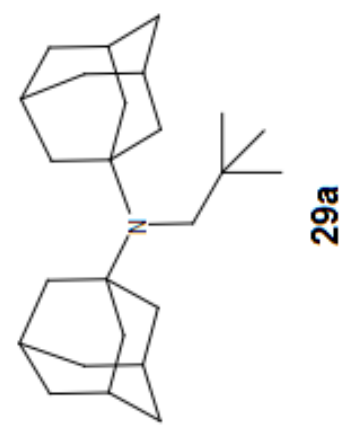

Figure S-54: ${ }^{13} \mathrm{C}\left\{{ }^{1} \mathrm{H}\right\}$ NMR spectrum of $29 \mathbf{a}$ in $\mathrm{C}_{6} \mathrm{D}_{6}$. 
हS' I

I9 1

29. 1

⒍ I

$10^{\circ} \mathrm{z}$

9I' 2

$\left.\begin{array}{l}6 b^{\prime} \varepsilon \\ Z S^{\prime} \varepsilon \\ Z S^{\prime} \varepsilon \\ t S^{\prime} \varepsilon \\ 9 S^{\prime} \varepsilon\end{array}\right]$

$\left.\begin{array}{l}66^{\prime} \varepsilon \\ \text { ZS' } \varepsilon \\ \text { ZS' } \varepsilon \\ \text { tS' } \varepsilon \\ 9 S^{\prime} \varepsilon\end{array}\right]$

$\left.\begin{array}{l}6 b^{\prime} \varepsilon \\ Z S^{\prime} \varepsilon \\ Z S^{\prime} \varepsilon \\ t S^{\prime} \varepsilon \\ 9 S^{\prime} \varepsilon\end{array}\right]$

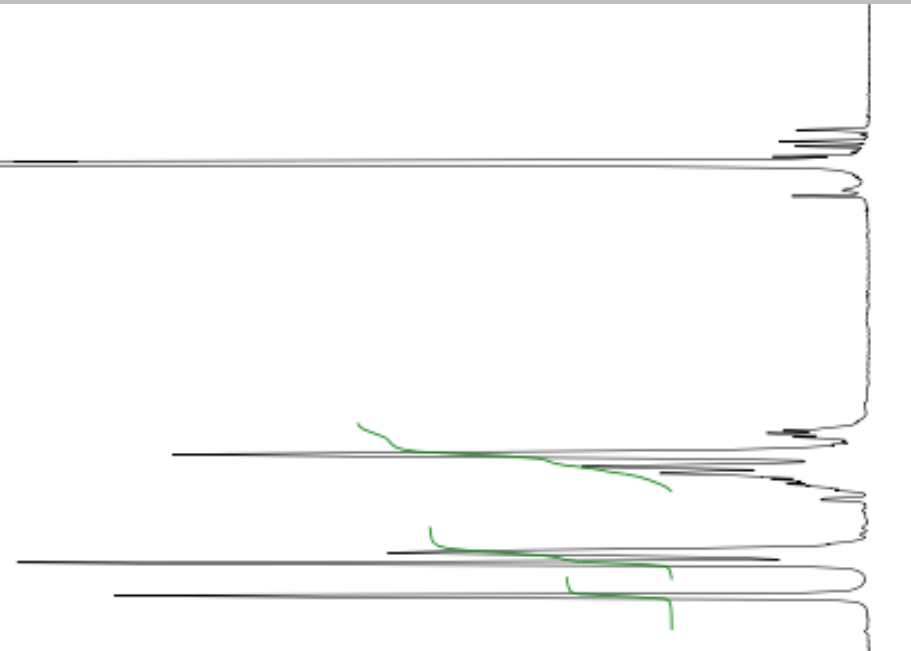

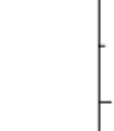

$\because$

?ำ

$\stackrel{\circ}{\rightarrow}$

L

1 -

$-00^{\circ} \mathrm{t}-\mathrm{i}$

$\stackrel{n}{\mathrm{n}}$

$\circ$

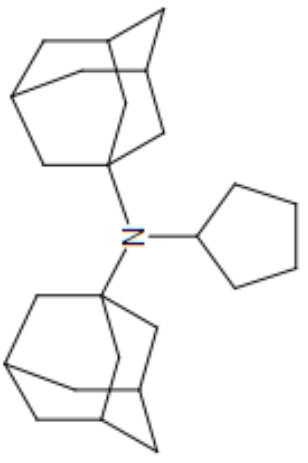

으ำ

Figure S-55: ${ }^{1} \mathrm{H}$ NMR spectrum of $29 b$ in $\mathrm{C}_{6} \mathrm{D}_{6}$. 


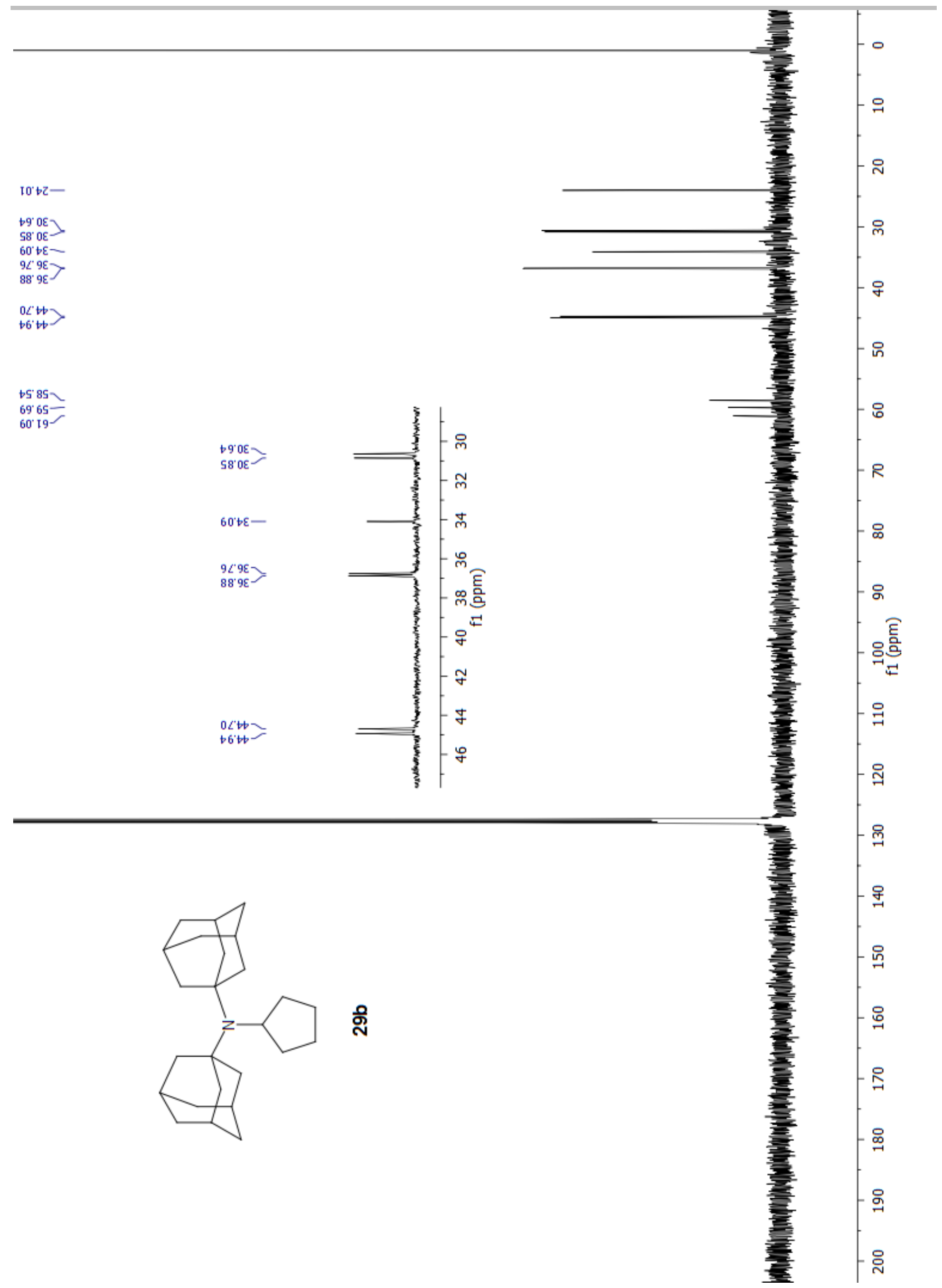

Figure S-56: ${ }^{13} \mathrm{C}\left\{{ }^{1} \mathrm{H}\right\}$ NMR spectrum of $29 \mathrm{~b}$ in $\mathrm{C}_{6} \mathrm{D}_{6}$. 


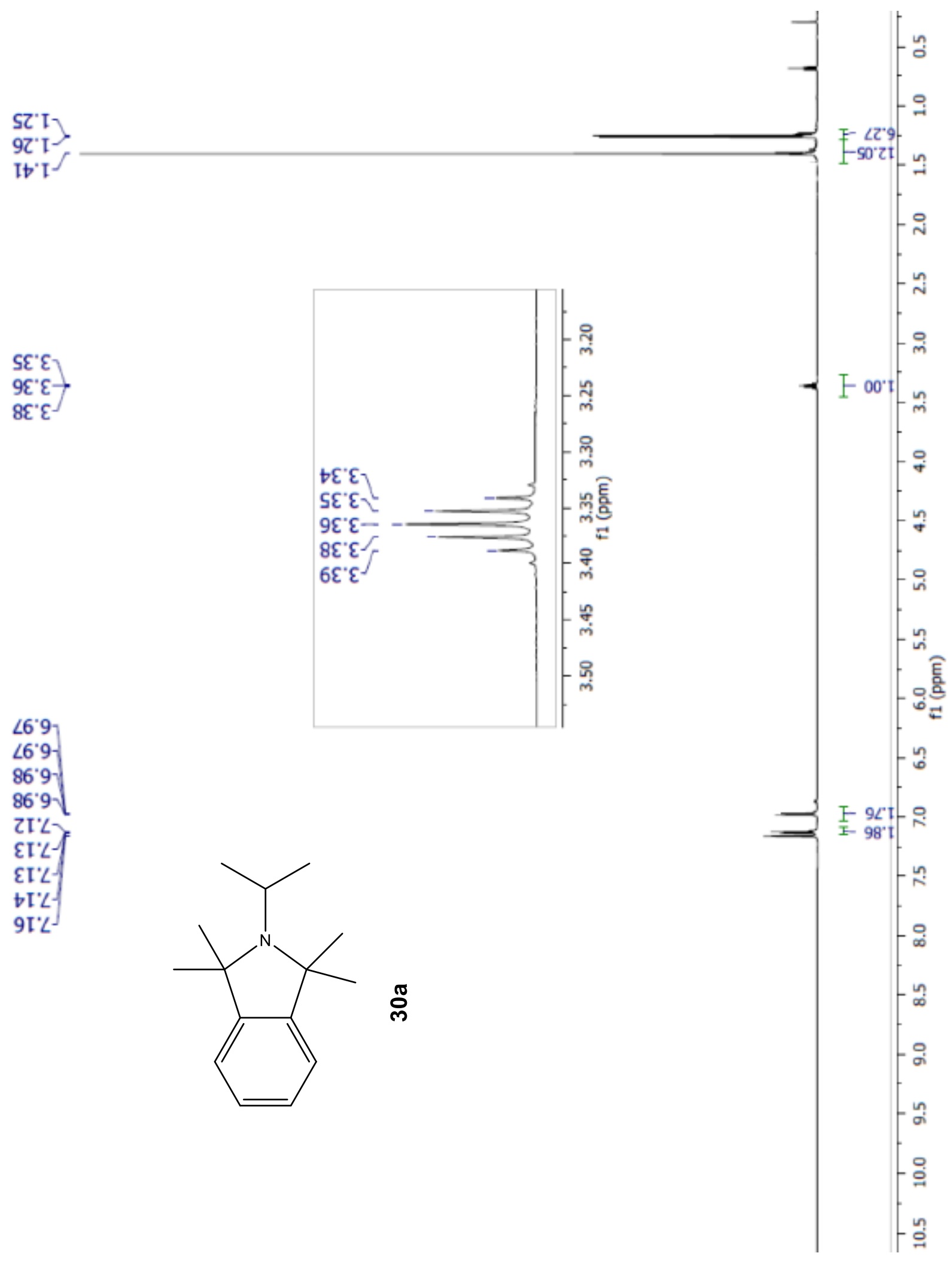

Figure S-57: ${ }^{1} \mathrm{H}$ NMR spectrum of $\mathbf{3 0 a}$ in $\mathrm{C}_{6} \mathrm{D}_{6}$. 


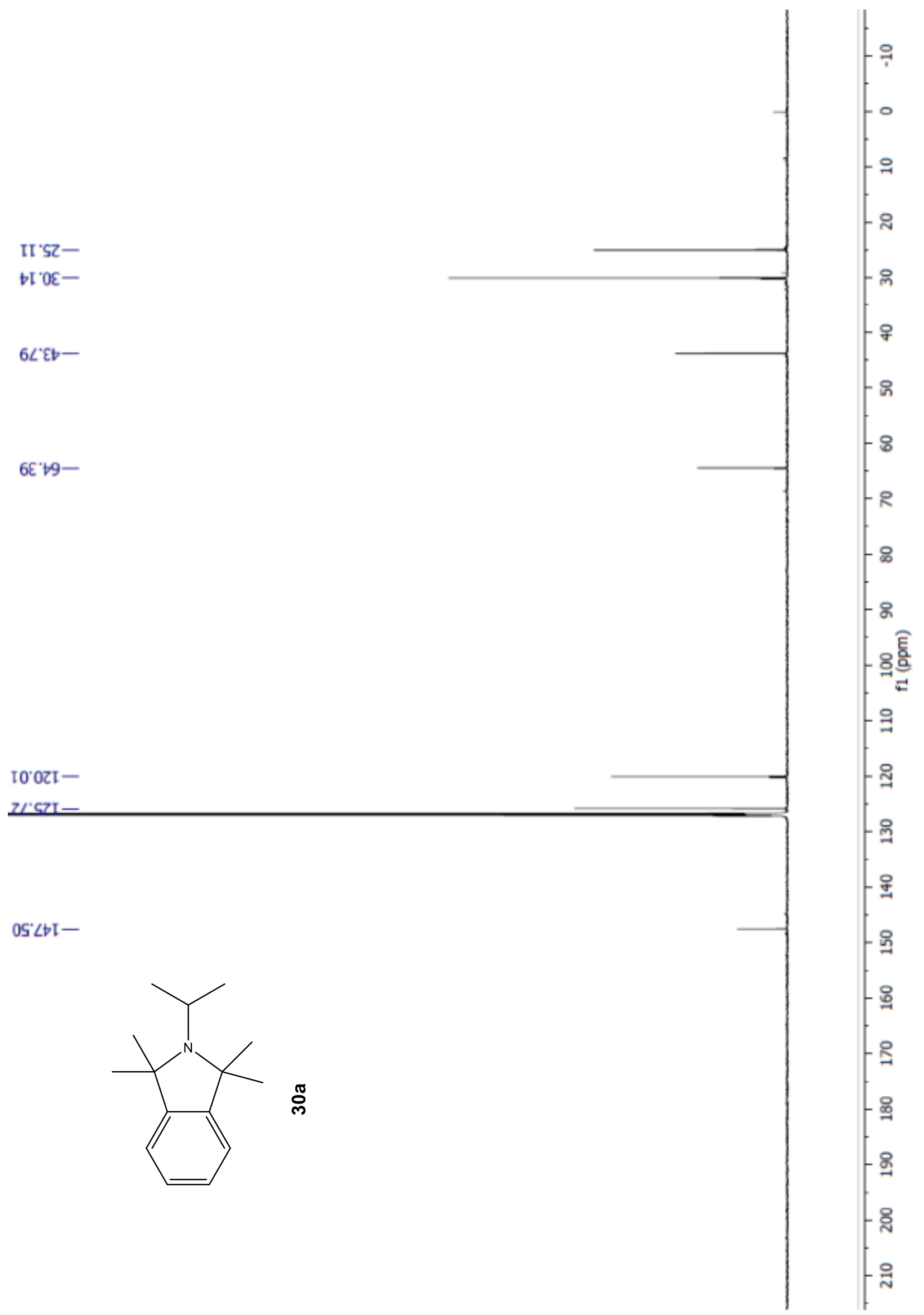

Figure S-58: ${ }^{13} \mathrm{C}\left\{{ }^{1} \mathrm{H}\right\}$ NMR spectrum of $30 \mathrm{a}$ in $\mathrm{C}_{6} \mathrm{D}_{6}$. 


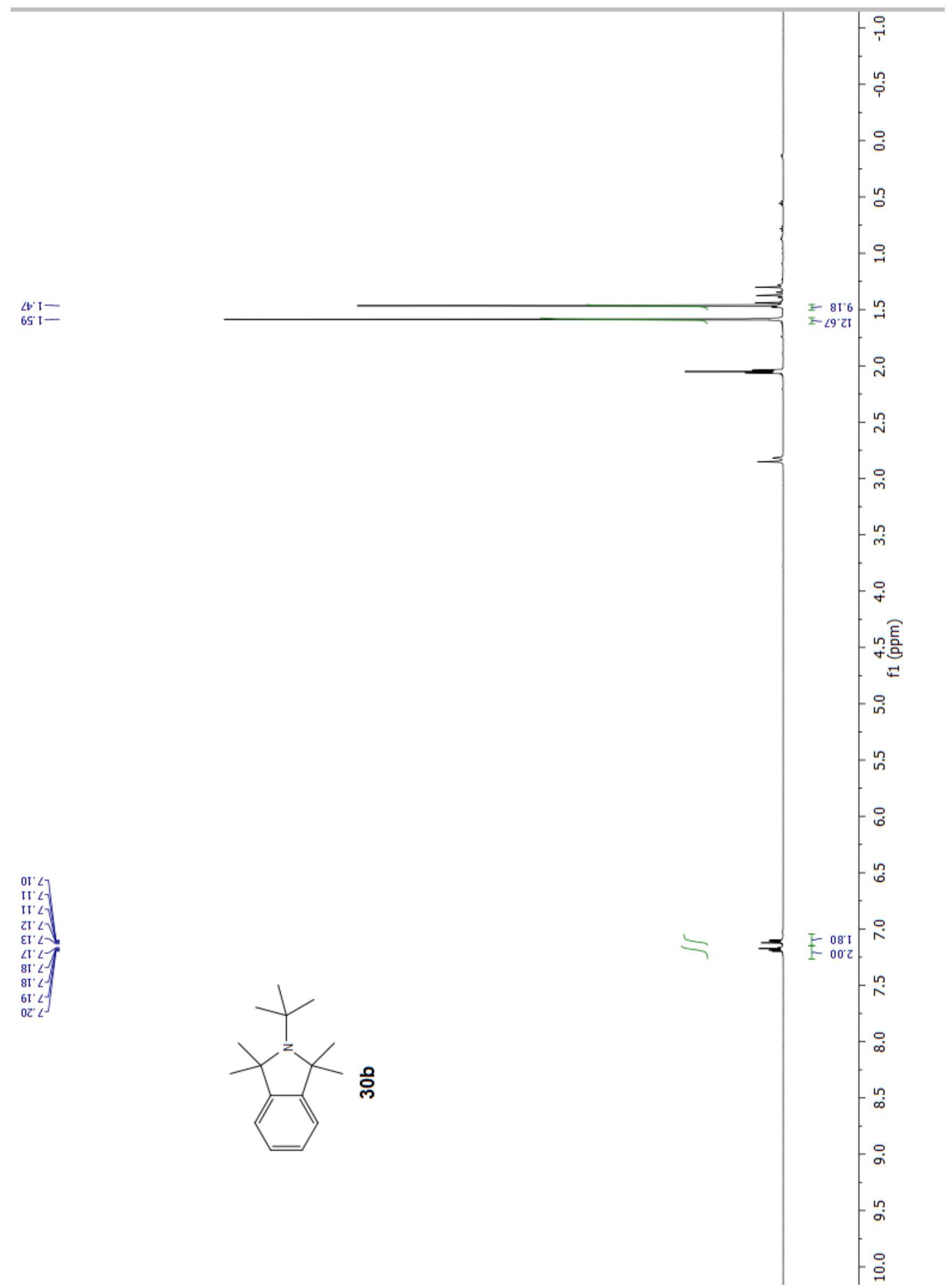

Figure S-59: ${ }^{1} \mathrm{H}$ NMR spectrum of $\mathbf{3 0 b}$ in $\left(\mathrm{CD}_{3}\right)_{2} \mathrm{CO}$. 


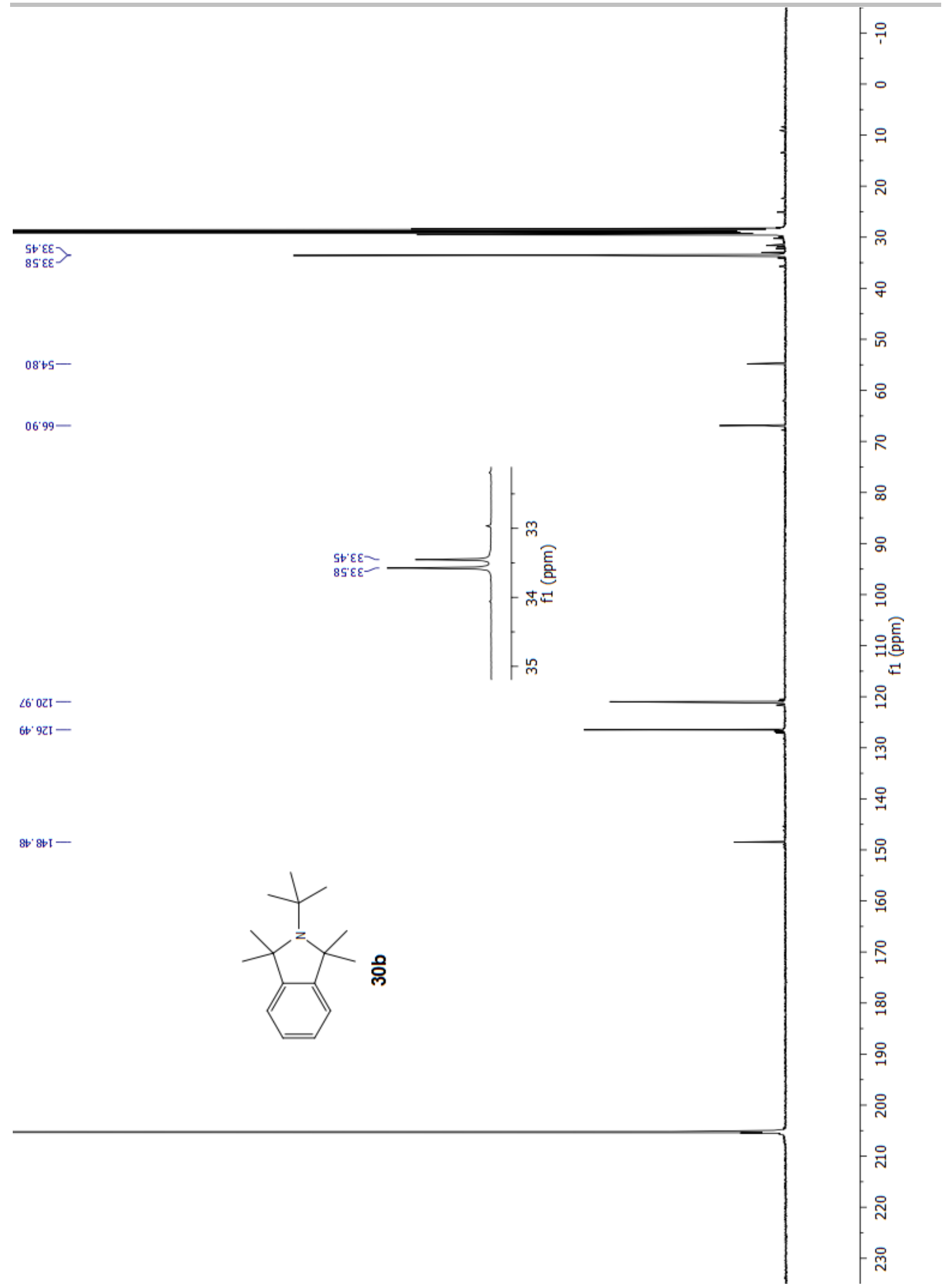

Figure S-60: ${ }^{13} \mathrm{C}\left\{{ }^{1} \mathrm{H}\right\}$ NMR spectrum of $\mathbf{3 0 b}$ in $\left(\mathrm{CD}_{3}\right)_{2} \mathrm{CO}$. 


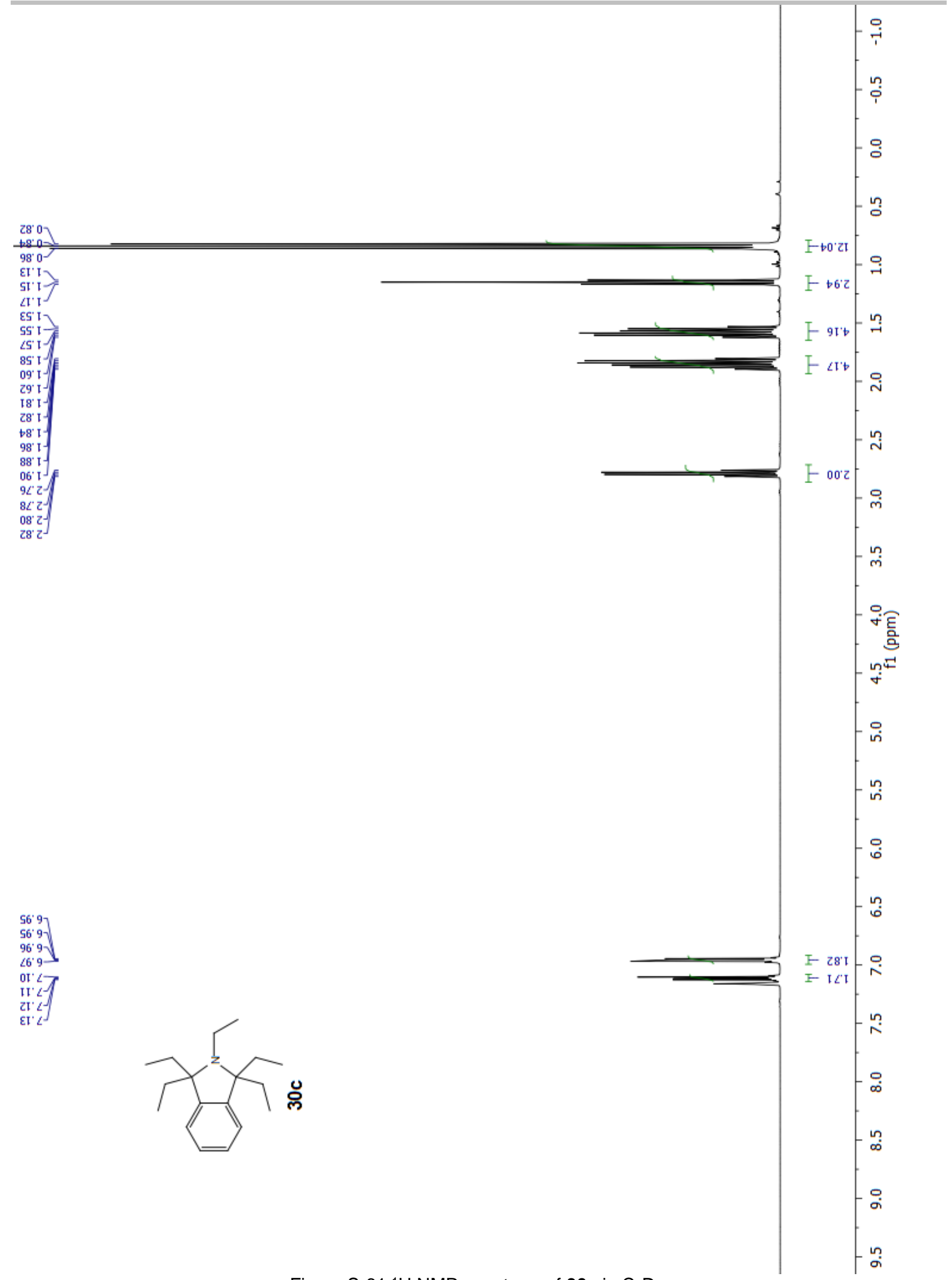

Figure S-61: ${ }^{1} \mathrm{H}$ NMR spectrum of $30 \mathrm{c}$ in $\mathrm{C}_{6} \mathrm{D}_{6}$. 
05' 6 -

ง8' 91 -

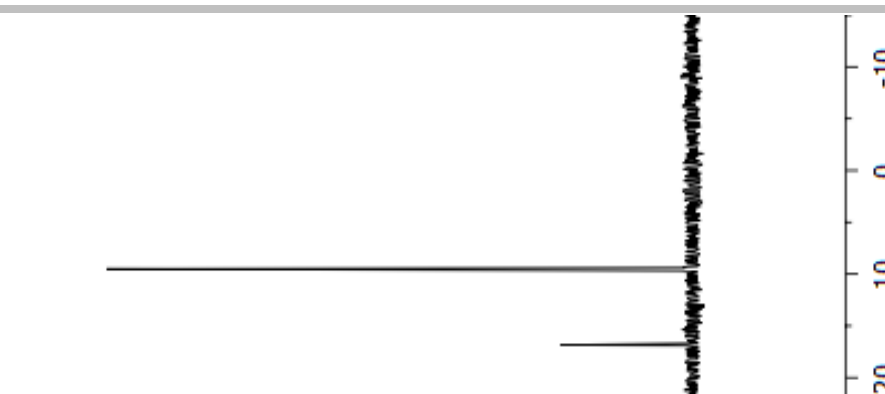

ع6. $0 \varepsilon-$

๑ $\varsigma \varepsilon-$

0S. $0<-$

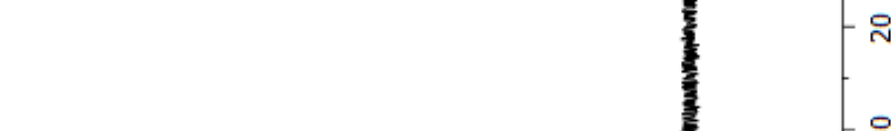

으

in

8

$8 C Z Z I-$

$\mathrm{b}<\mathrm{szI}-$

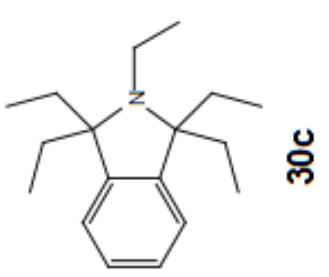

Figure S-62: ${ }^{13} \mathrm{C}\left\{{ }^{1} \mathrm{H}\right\}$ NMR spectrum of $30 \mathrm{c}$ in $\mathrm{C}_{6} \mathrm{D}_{6}$.

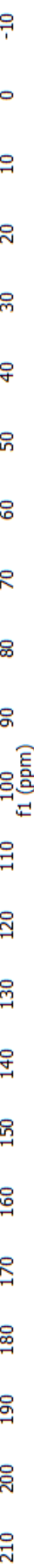

옥 


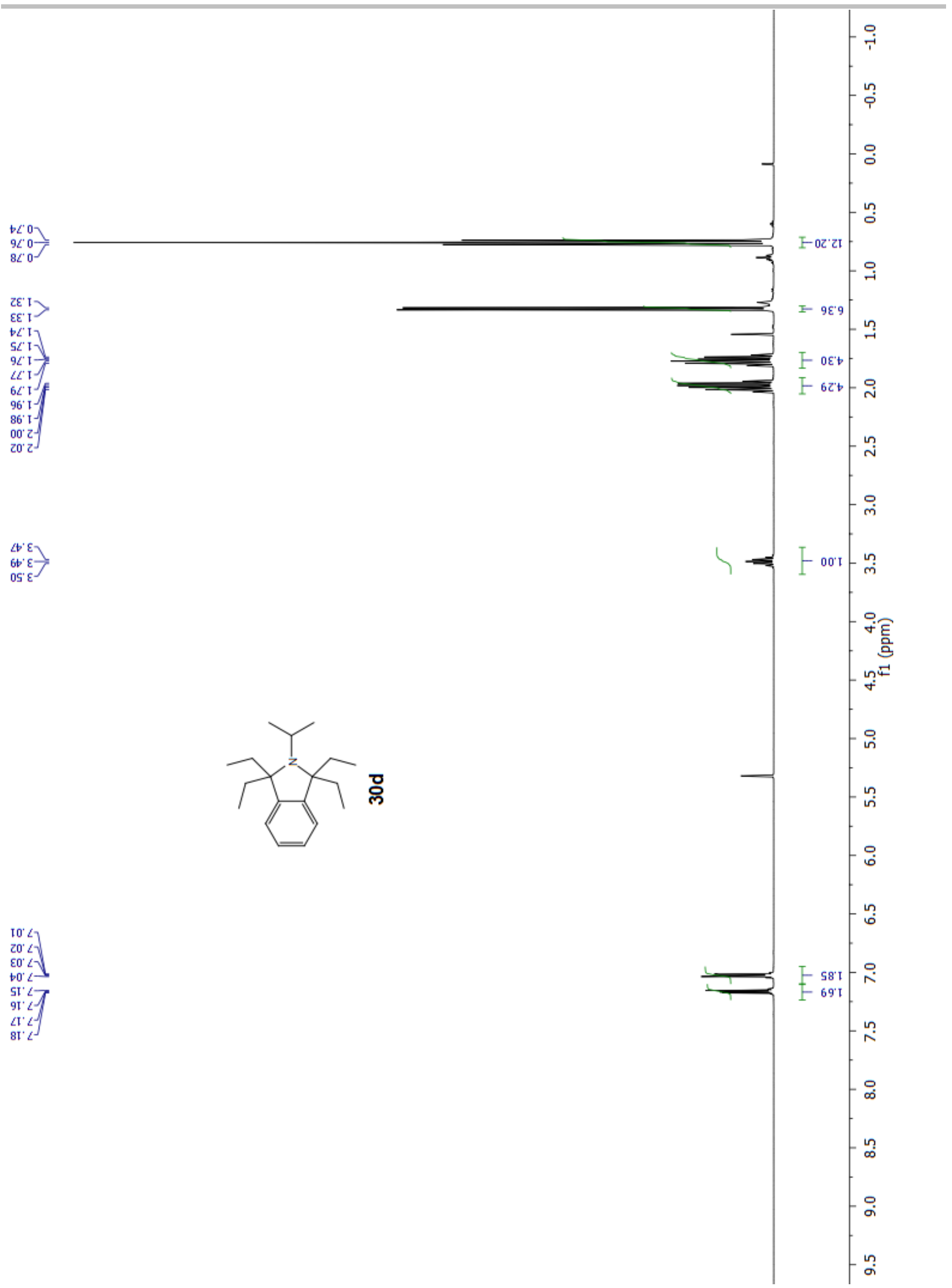

Figure S-63: ${ }^{1} \mathrm{H}$ NMR spectrum of $30 \mathrm{~d}$ in $\mathrm{CD}_{2} \mathrm{Cl}_{2}$. 


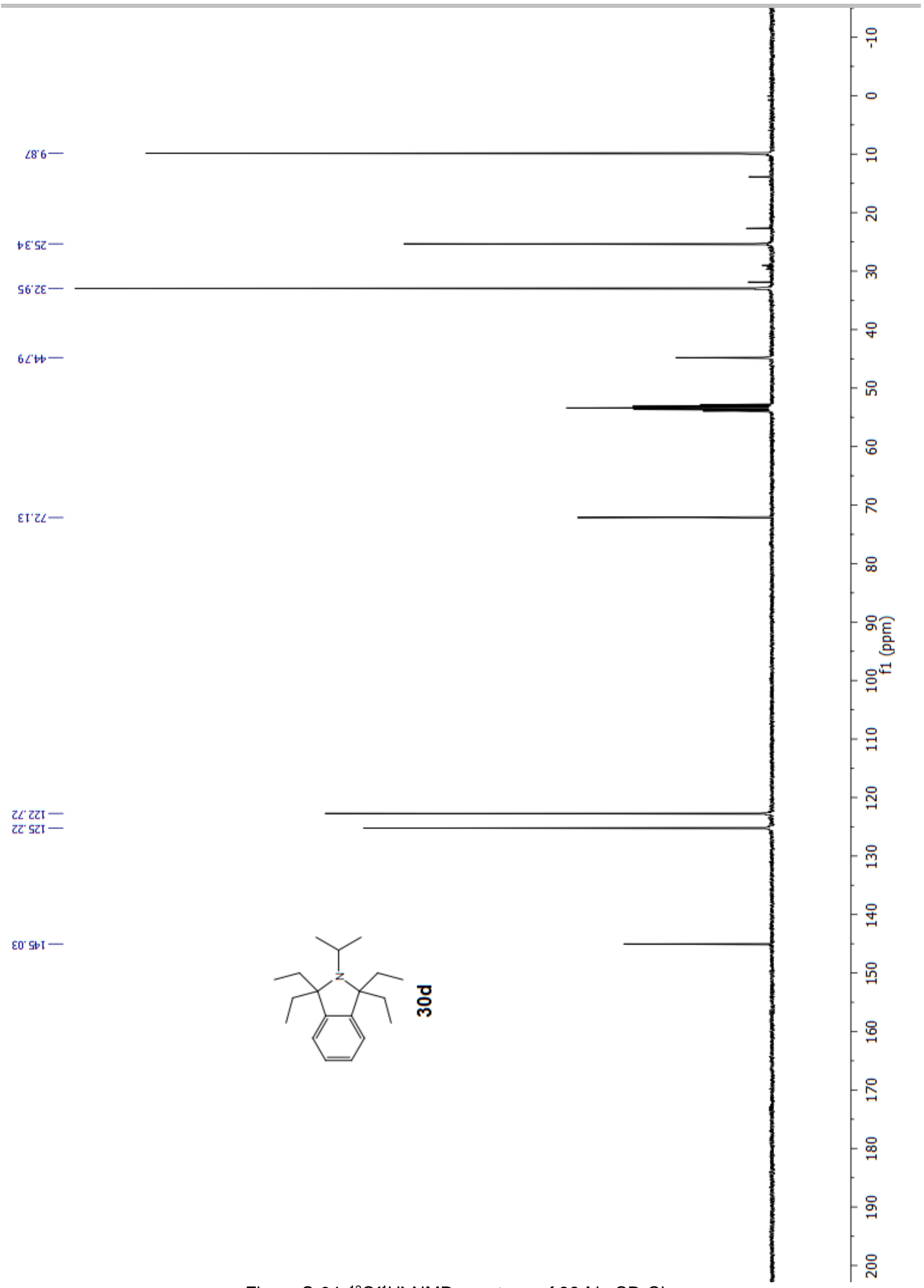

Figure S-64: ${ }^{13} \mathrm{C}\left\{{ }^{1} \mathrm{H}\right\}$ NMR spectrum of $30 \mathrm{~d}$ in $\mathrm{CD}_{2} \mathrm{Cl}_{2}$. 

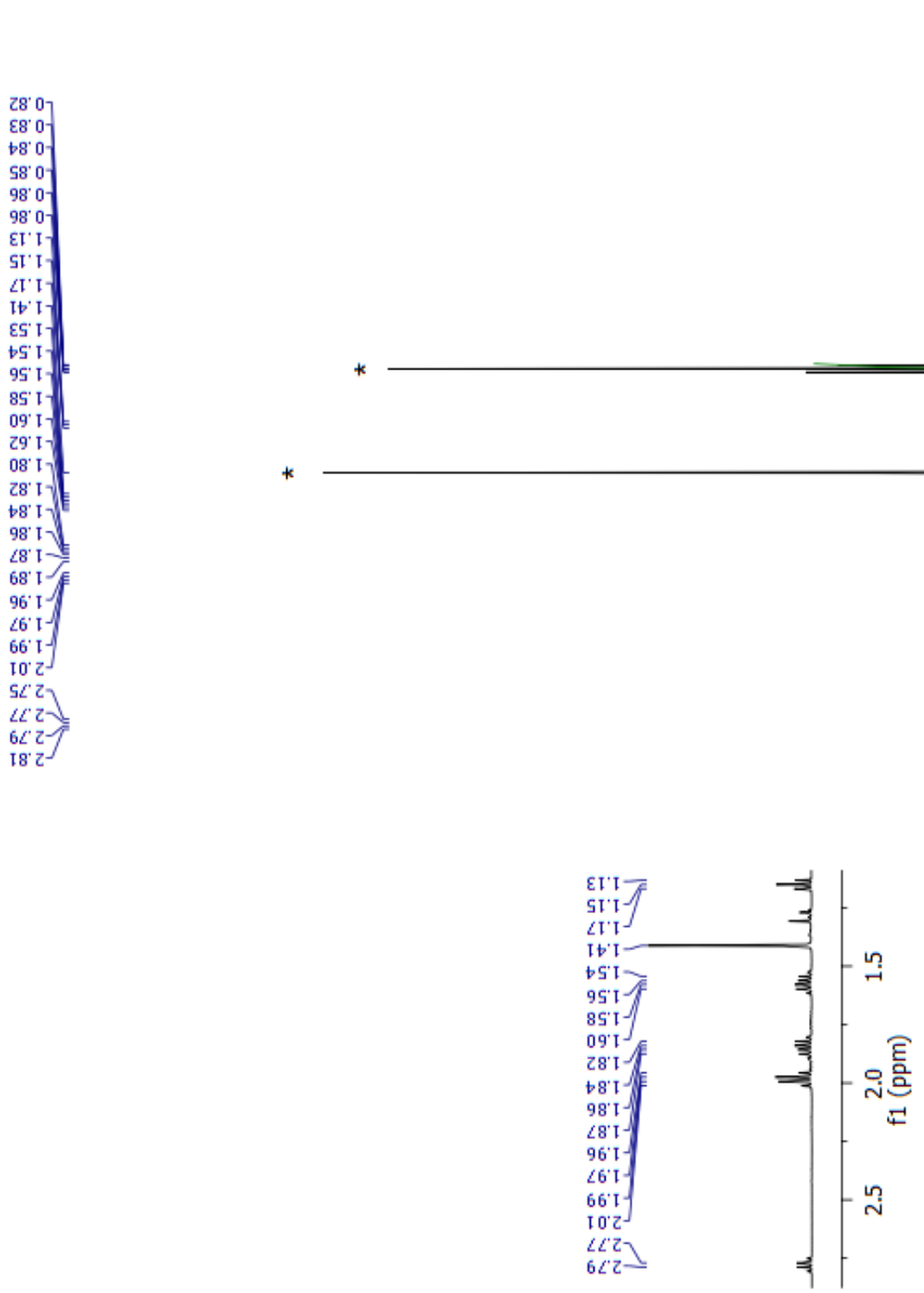

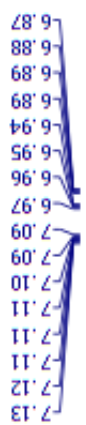

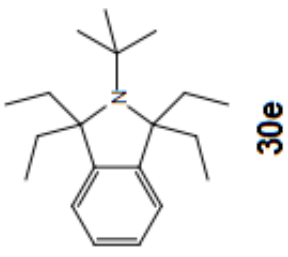

今్
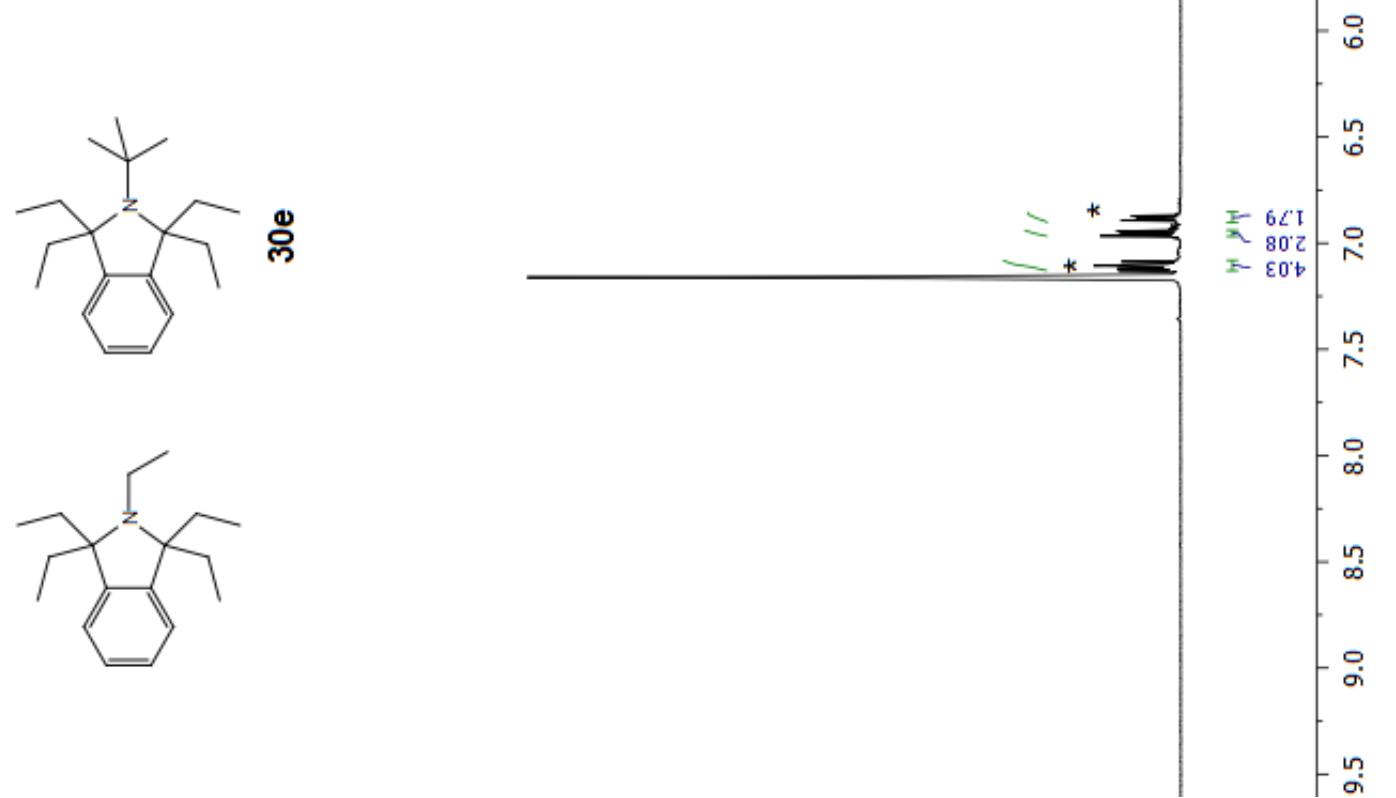

Figure S-65: ${ }^{1} \mathrm{H}$ NMR spectrum of $\mathbf{3 0 e}$ [marked signals $\left({ }^{*}\right)$ ] and 1,1,2,3,3-pentaethylisoindoline as a 1:1.2 mixture in $\mathrm{C}_{6} \mathrm{D}_{6}$. 

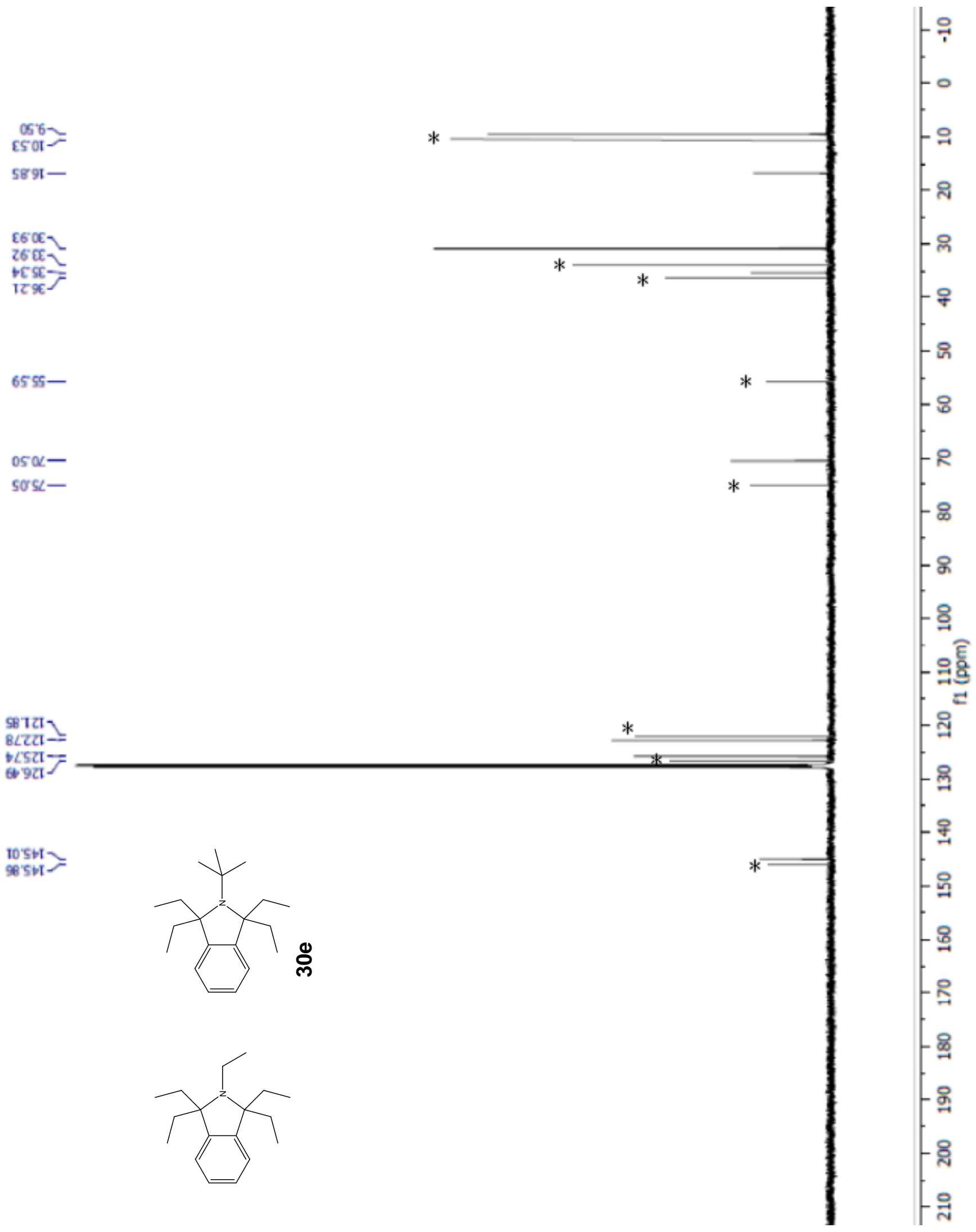

$-\frac{7}{1}$

$05 \alpha-$

soss-

SBTZI)

$8 \angle 2 C 1=$

${ }_{6}^{+} \mathrm{LSCl}-\overline{5}$

10 ' $5+1=$
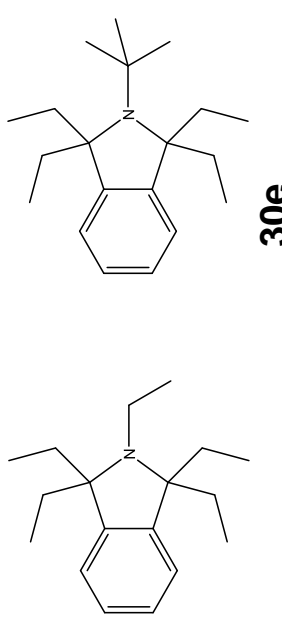

요

웅

은

Figure S-66: ${ }^{13} \mathrm{C}\left\{{ }^{1} \mathrm{H}\right\}$ NMR spectrum of $\mathbf{3 0 e}$ [marked signals $\left.\left(^{*}\right)\right]$ and 1,1,2,3,3-pentaethylisoindoline as a 1:1.2 mixture in $\mathrm{C}_{6} \mathrm{D}_{6}$. 


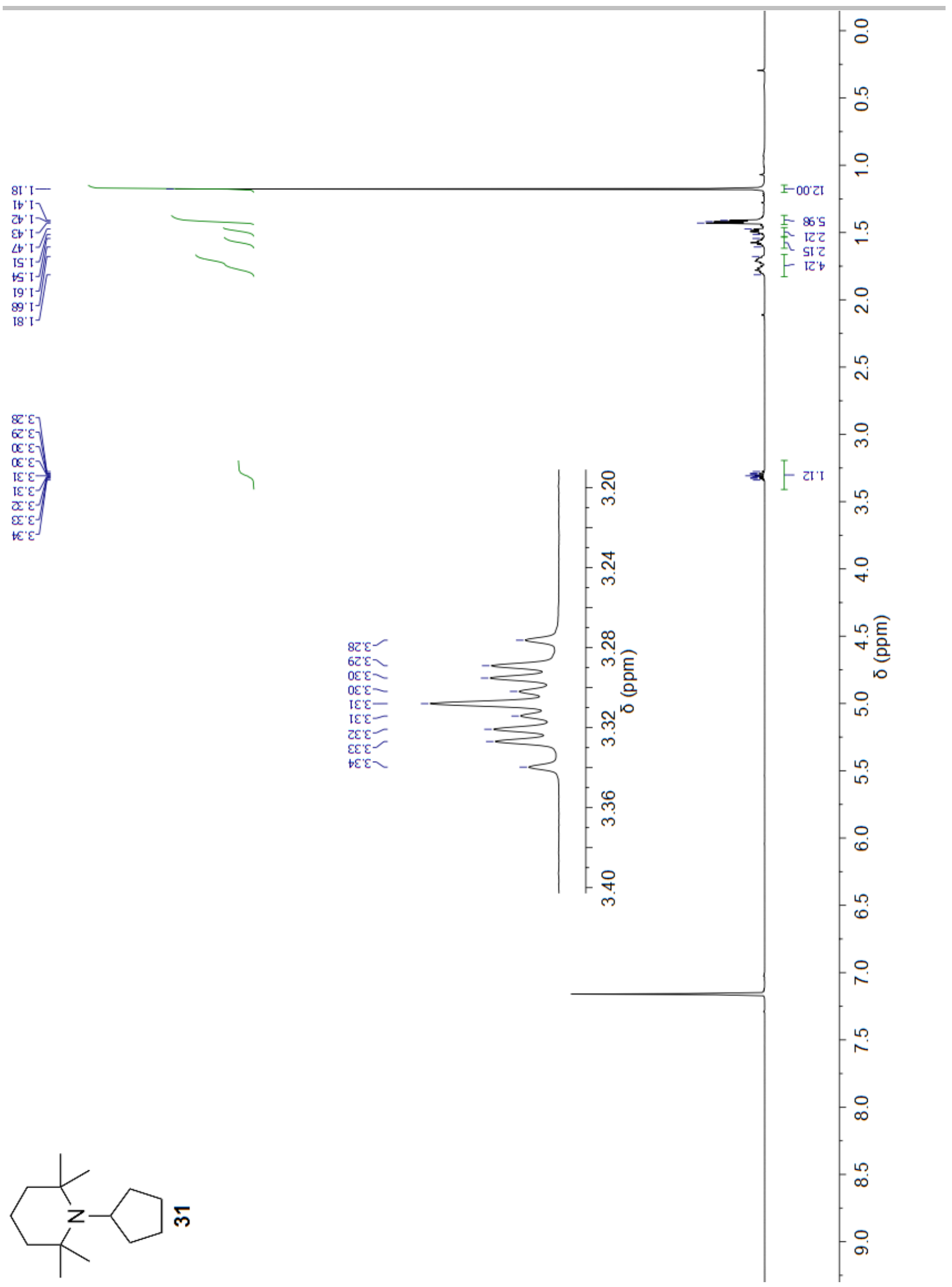

Figure S-67: ${ }^{1} \mathrm{H}$ NMR spectrum of 31 in $\mathrm{C}_{6} \mathrm{D}_{6}$. 


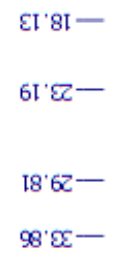

to'th-

$16 \cdot t 5-$

$26 \cdot 65-$

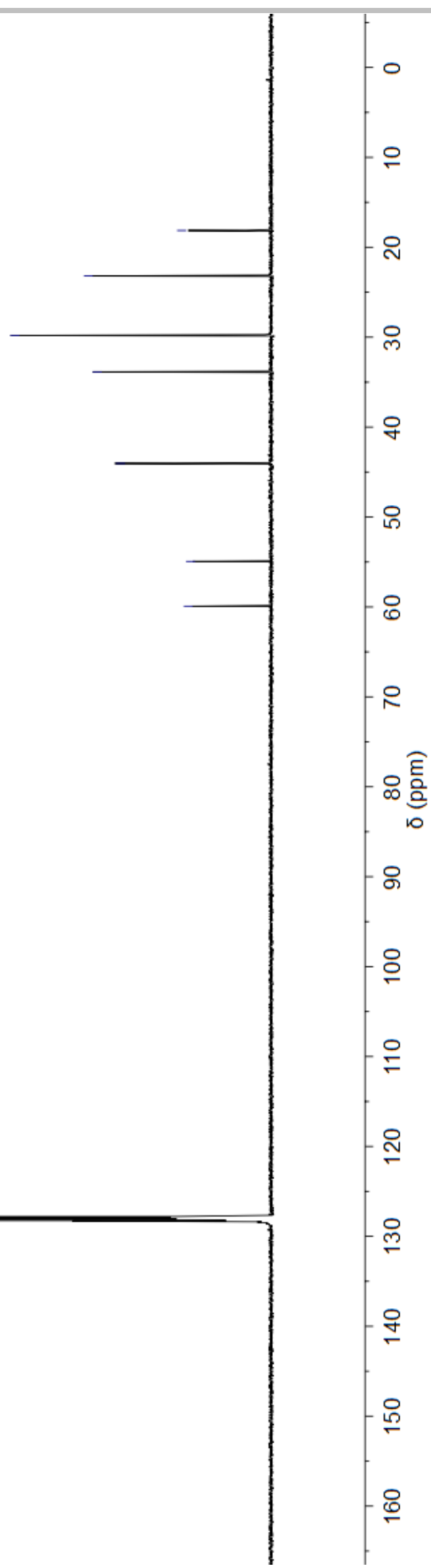

Figure S-68: ${ }^{13} \mathrm{C}\left\{{ }^{1} \mathrm{H}\right\}$ NMR spectrum of 31 in $\mathrm{C}_{6} \mathrm{D}_{6}$. 


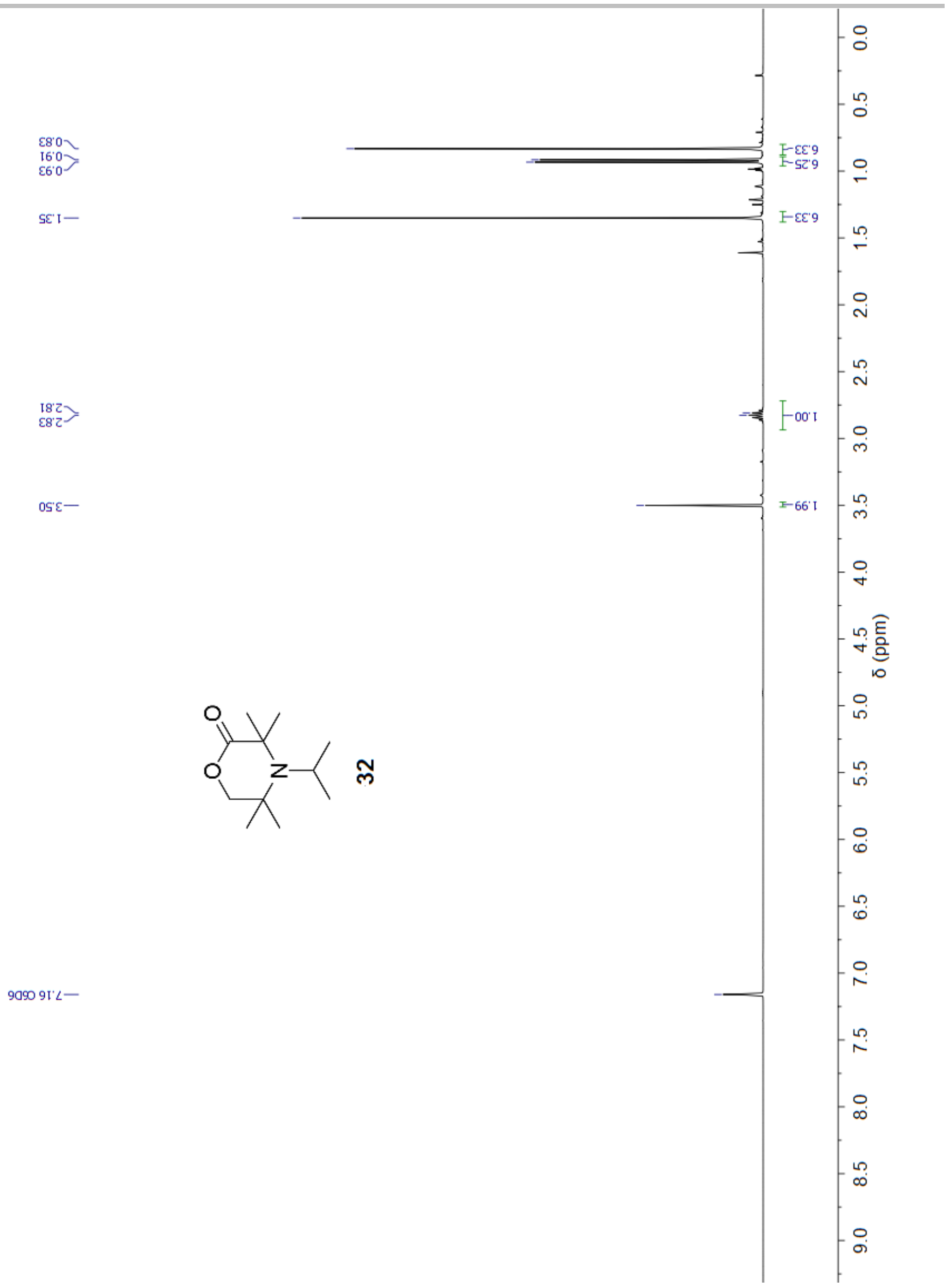

Figure S-69: ${ }^{1} \mathrm{H}$ NMR spectrum of 32 in $\mathrm{C}_{6} \mathrm{D}_{6}$. 


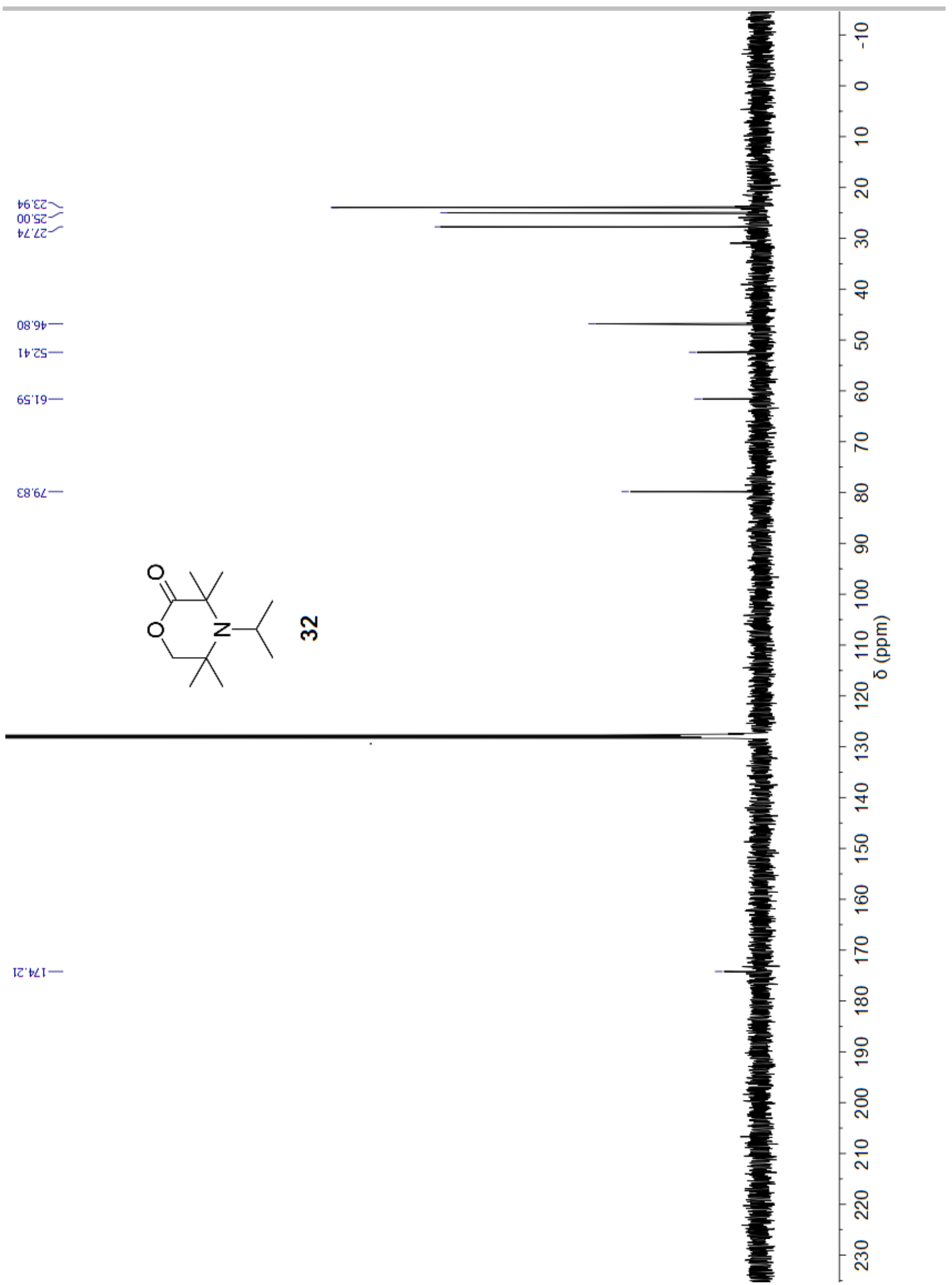

Figure S-70: ${ }^{13} \mathrm{C}\left\{{ }^{1} \mathrm{H}\right\}$ NMR spectrum of 32 in $\mathrm{C}_{6} \mathrm{D}_{6}$. 


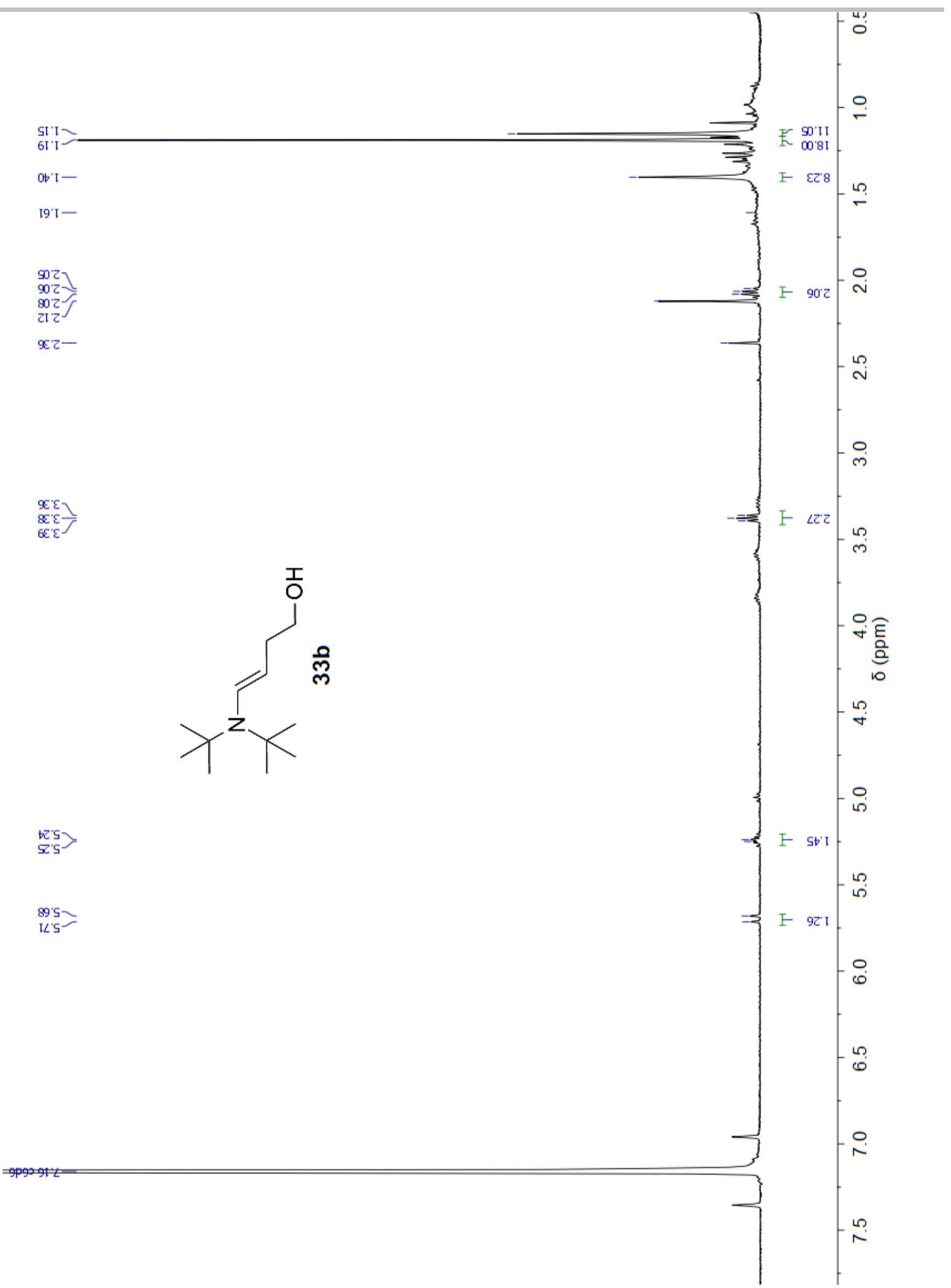

Figure S-71: ${ }^{1} \mathrm{H}$ NMR spectrum of $\mathbf{3 3 b}$ in $\mathrm{C}_{6} \mathrm{D}_{6}$. 


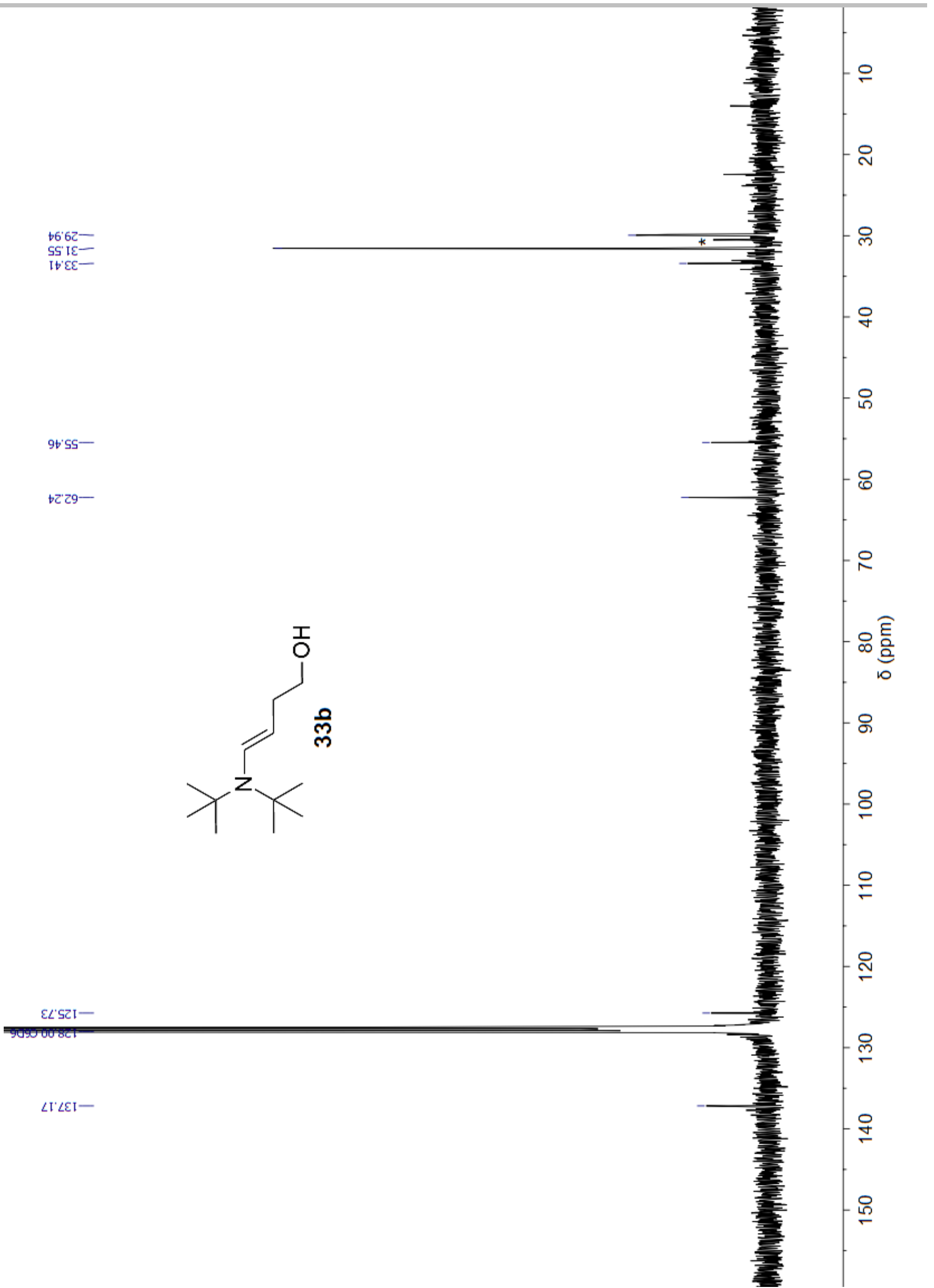

Figure S-72: ${ }^{13} \mathrm{C}\left\{{ }^{1} \mathrm{H}\right\}$ NMR spectrum of $\mathbf{3 3 b}$ in $\mathrm{C}_{6} \mathrm{D}_{6}$. The spectrum includes a signal of di-tert-butylamine $\left({ }^{*}\right)$. 


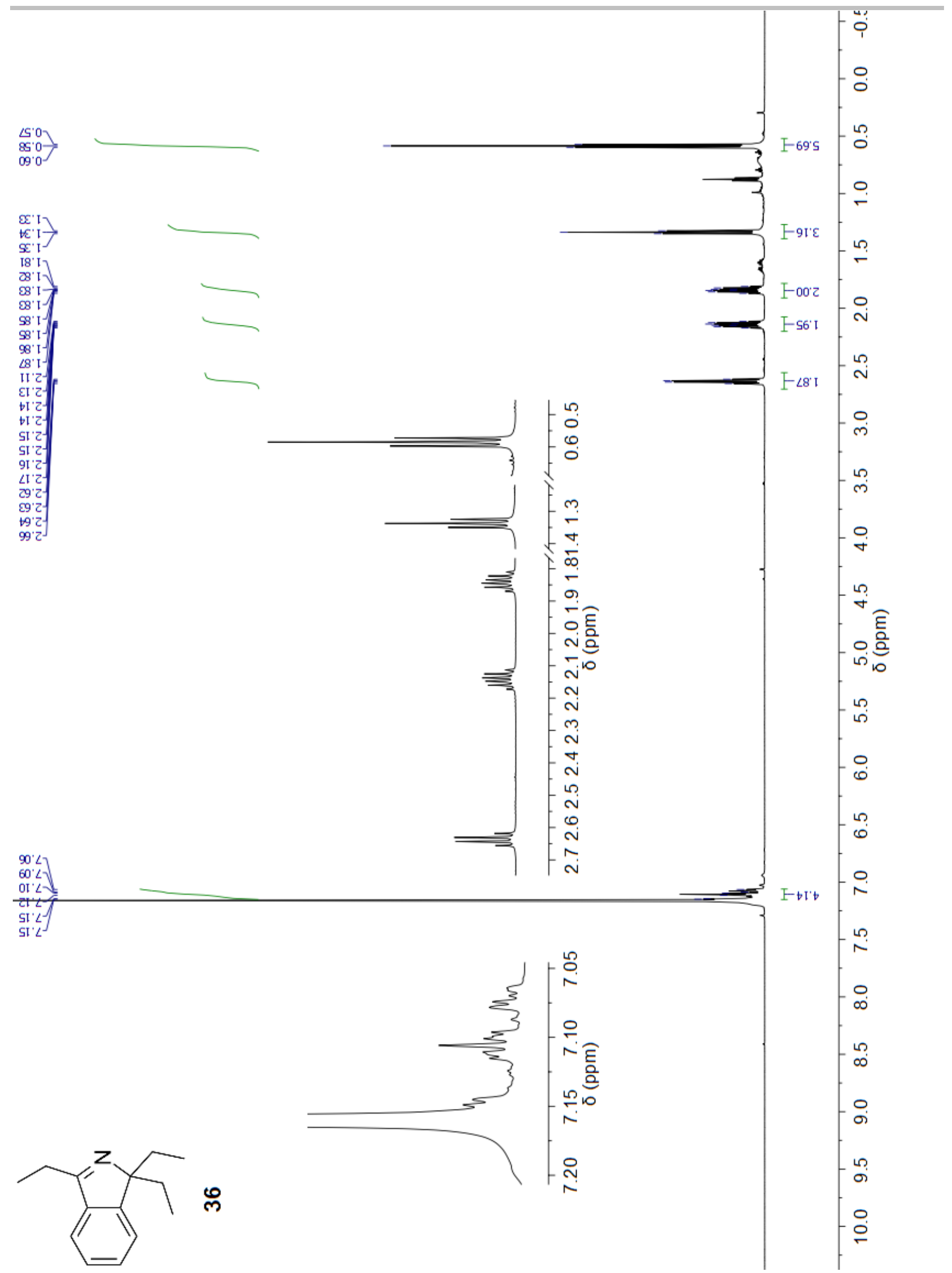

Figure S-73: ${ }^{1} \mathrm{H}$ NMR spectrum of 36 in $\mathrm{C}_{6} \mathrm{D}_{6}$. 
92. $8-$

$\angle t$ II -

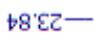

$96 \cdot 0 \varepsilon-$

$86 \cdot 08-$

$86^{\prime} 9 \mathrm{ZI}-$

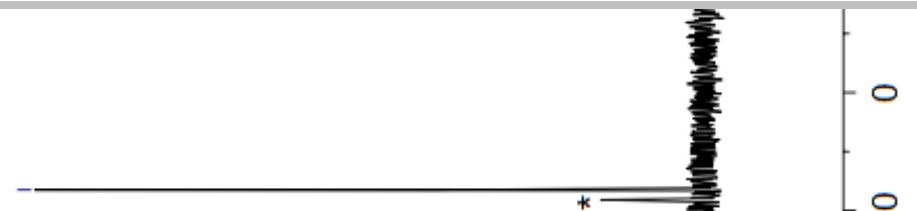

$99^{\circ}$ CZI

$09^{\circ} \mathrm{IZI}-$

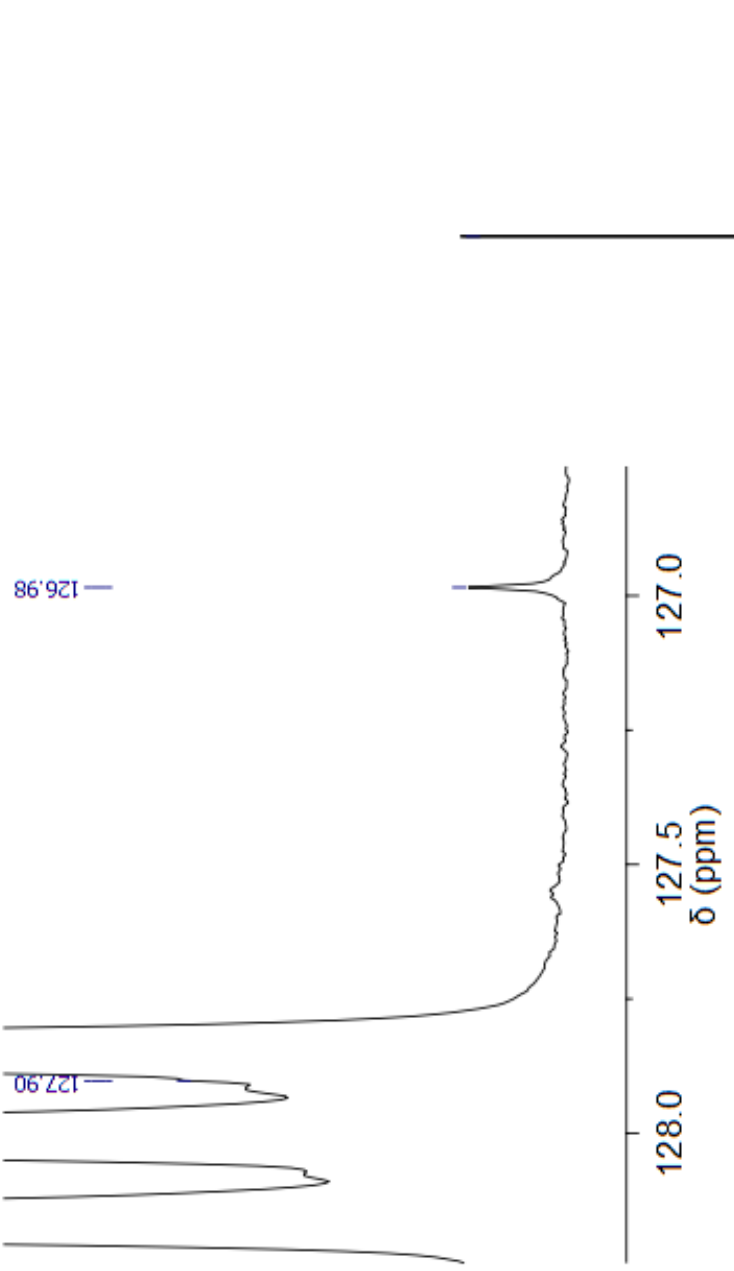

$86^{\circ} \mathrm{CI}-$

ก.

$\stackrel{2}{\simeq}$

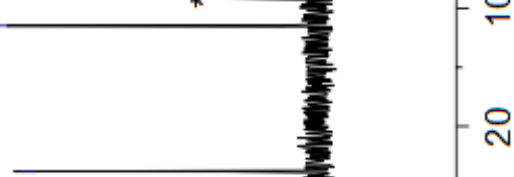

ㅇ

요

우

으

$0 \mathrm{CZT}$

It $00 \mathrm{I}$

8S'9SI-

Z'ZLI-

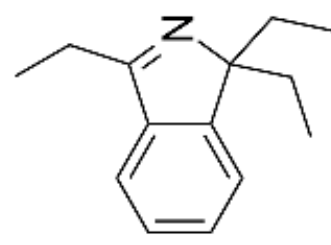

이

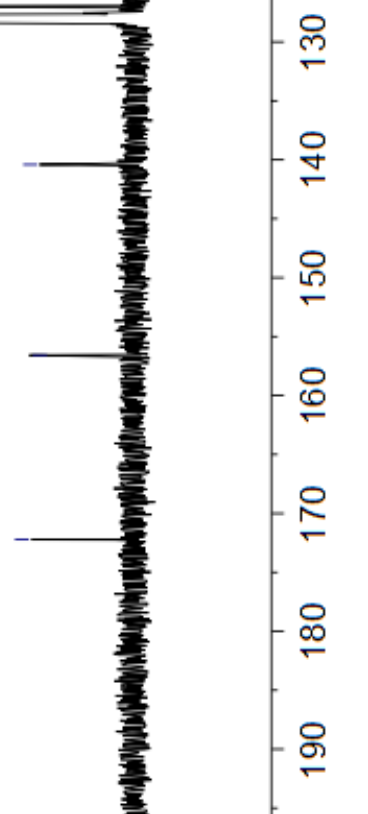

Figure S-74: ${ }^{13} \mathrm{C}\left\{{ }^{1} \mathrm{H}\right\}$ NMR spectrum of 36 in $\mathrm{C}_{6} \mathrm{D}_{6}$. The spectrum includes a signal $\left({ }^{*}\right)$ of an unknown impurity. 


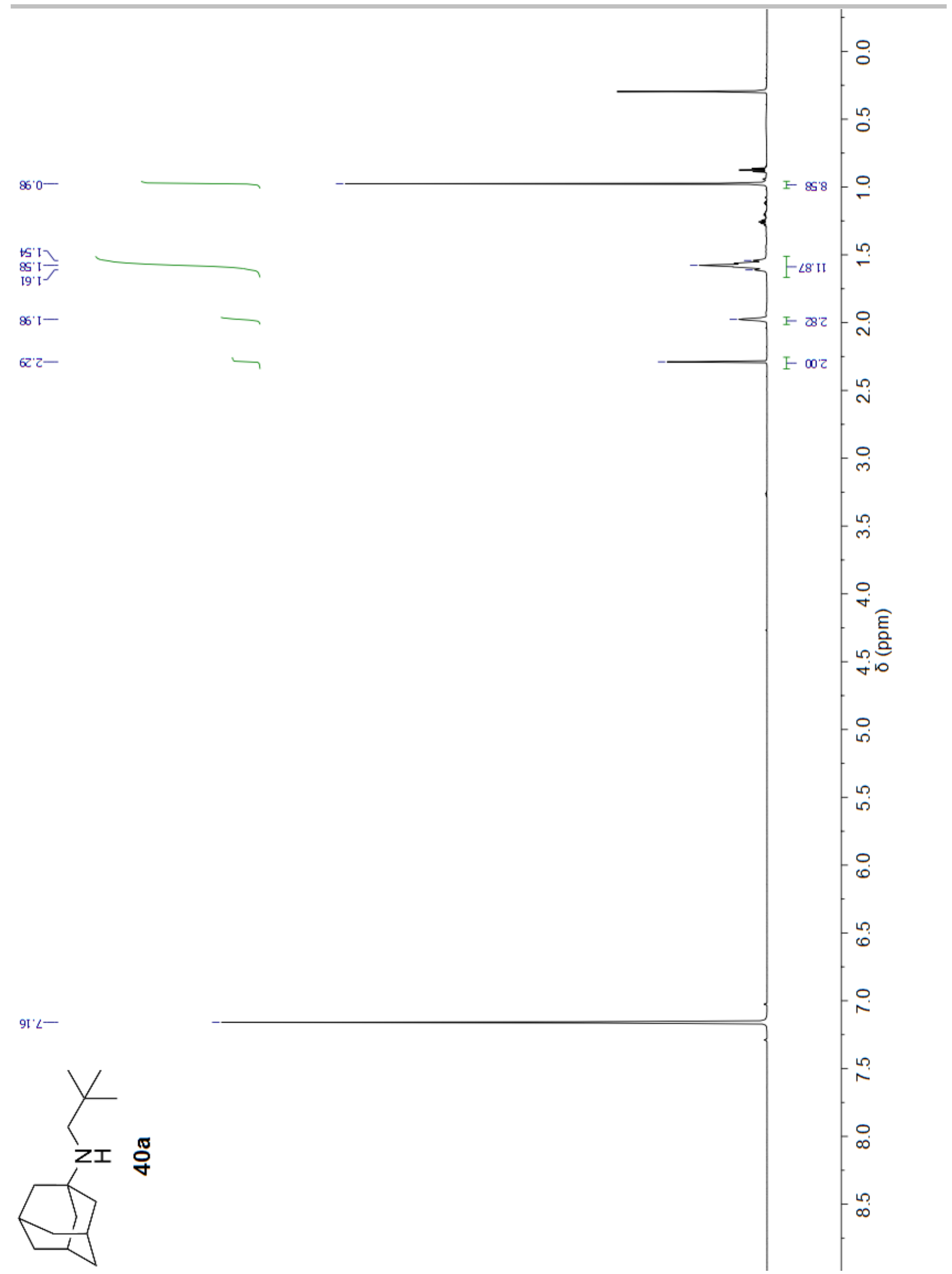

Figure S-75: ${ }^{1} \mathrm{H}$ NMR spectrum of $40 \mathrm{a}$ in $\mathrm{C}_{6} \mathrm{D}_{6}$. 


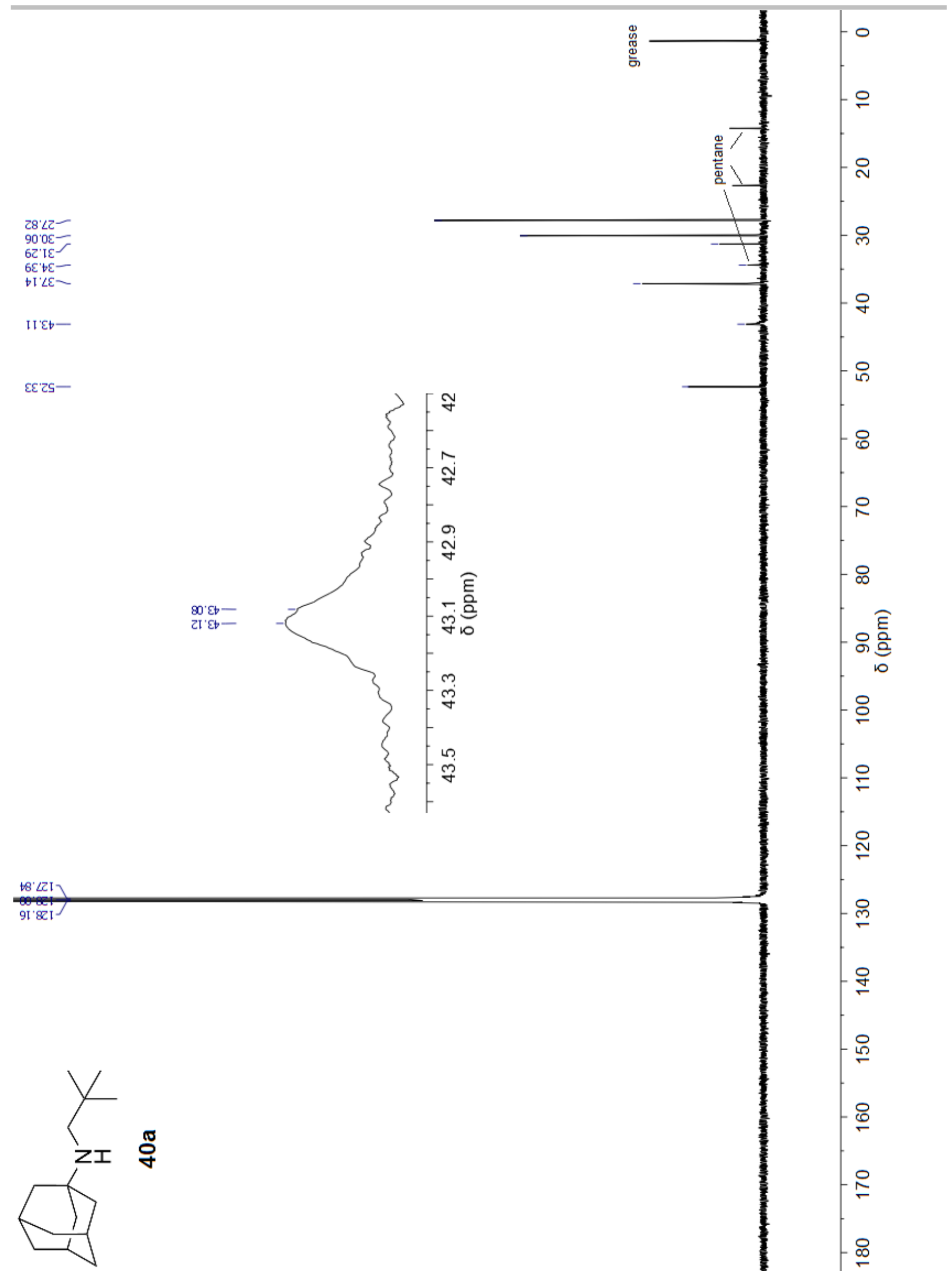

Figure S-76: ${ }^{13} \mathrm{C}\left\{{ }^{1} \mathrm{H}\right\}$ NMR spectrum of $40 \mathrm{a}$ in $\mathrm{C}_{6} \mathrm{D}_{6}$. 


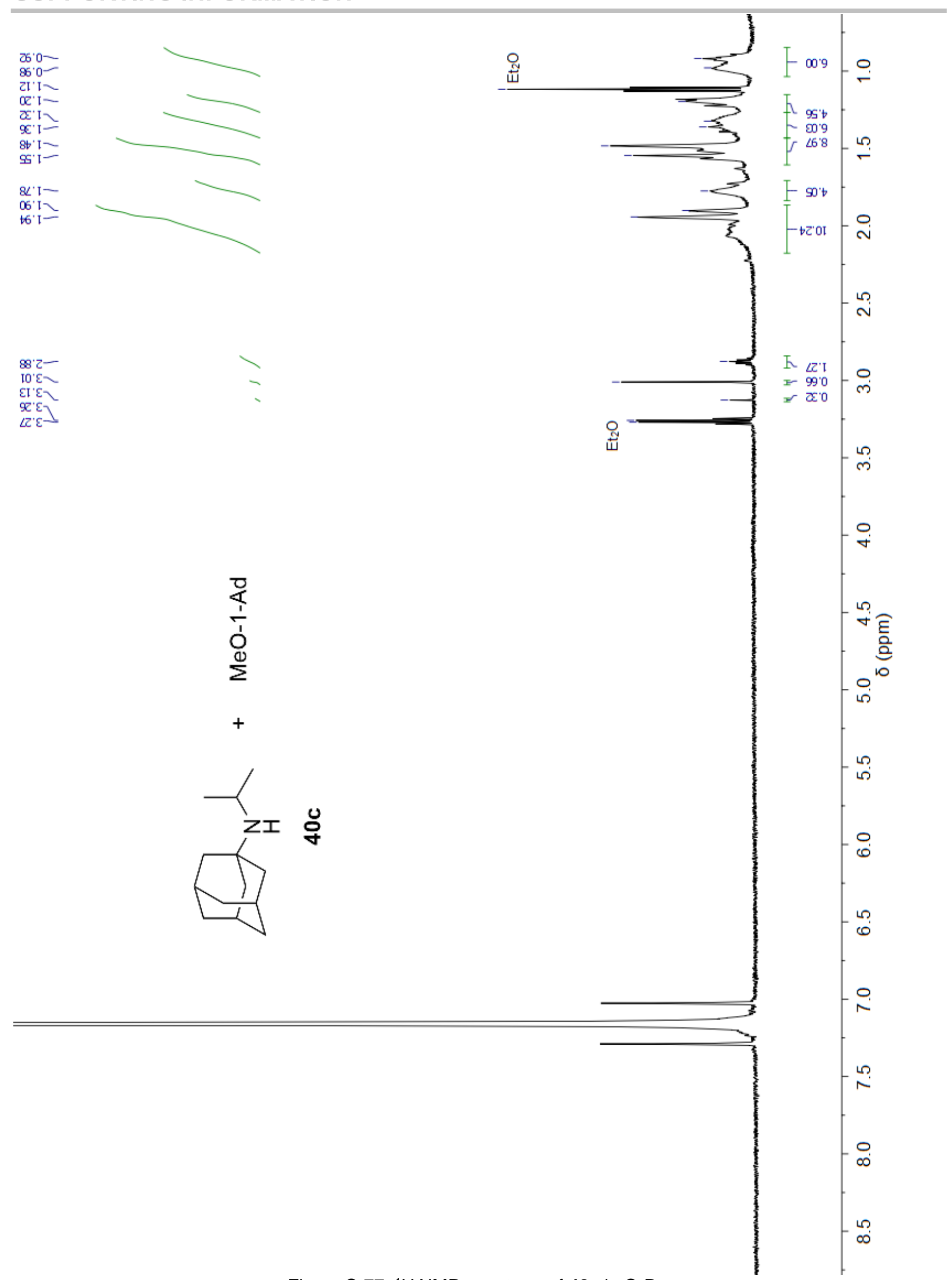

Figure S-77: ${ }^{1} \mathrm{H}$ NMR spectrum of $40 \mathrm{c}$ in $\mathrm{C}_{6} \mathrm{D}_{6}$. 


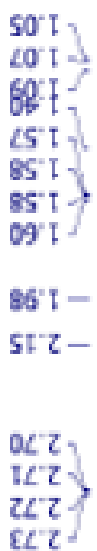

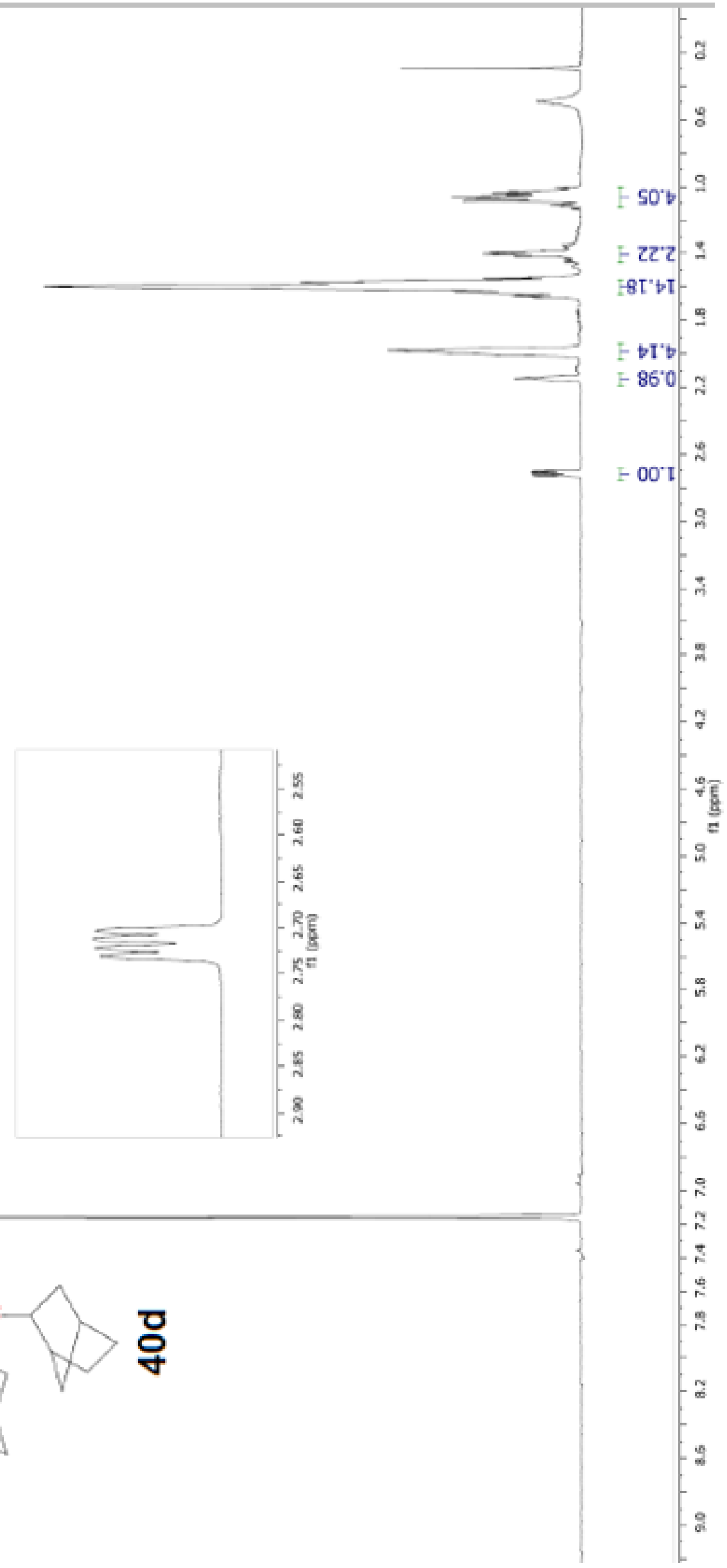

Figure S-78: ${ }^{1} \mathrm{H}$ NMR spectrum of $\mathbf{4 0 d}$ in $\mathrm{C}_{6} \mathrm{D}_{6}$. 


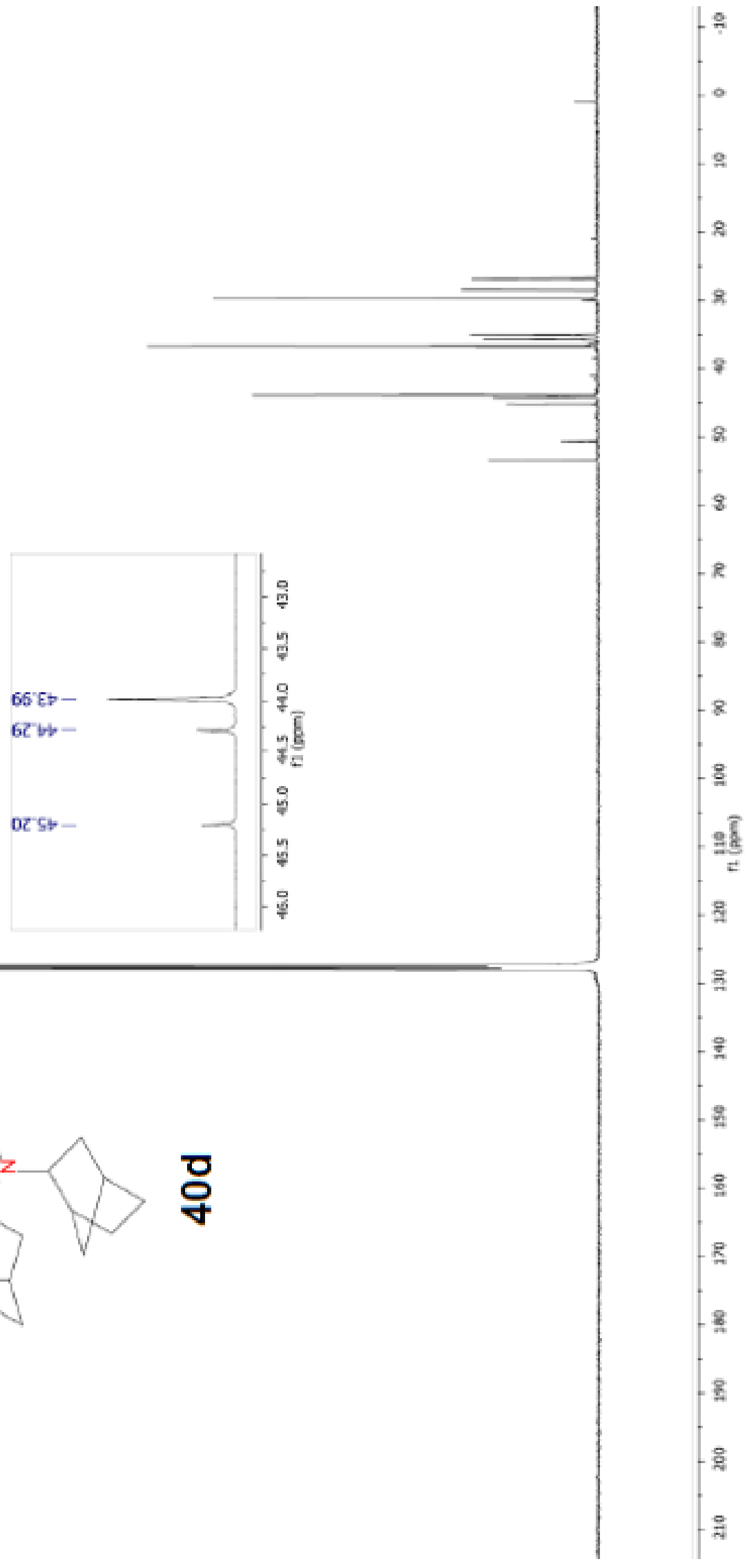

Figure S-79: ${ }^{13} \mathrm{C}\left\{{ }^{1} \mathrm{H}\right\}$ NMR spectrum of $40 \mathrm{~d}$ in $\mathrm{C}_{6} \mathrm{D}_{6}$. 


\section{Kinetic Data}

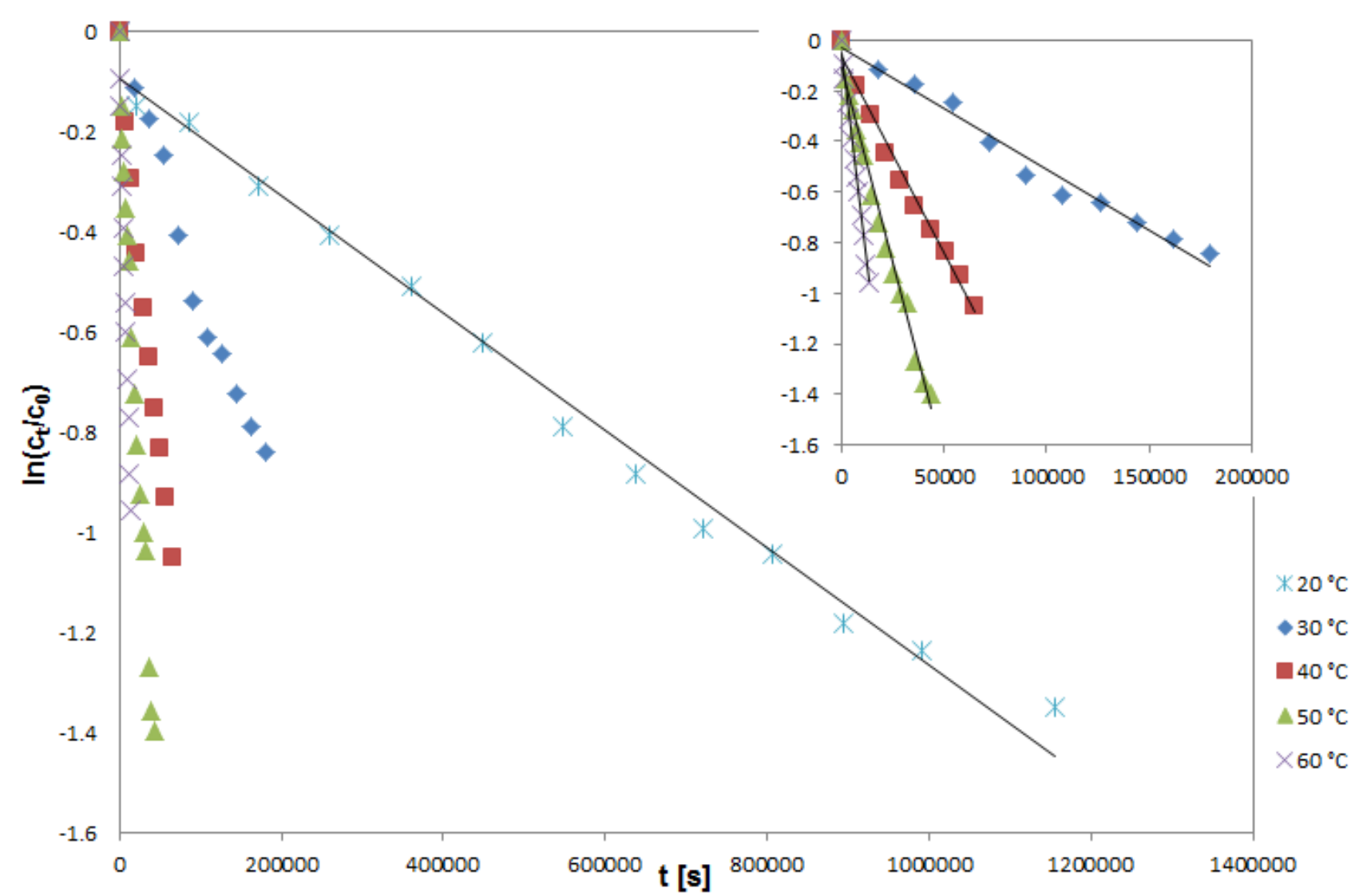

Figure S-80: $k$-values of the elimination reaction of 8 at different temperatures.

Table S-1: Ink- and half-life values of 8 at different temperatures.

\begin{tabular}{c|c|c|c} 
& $T_{1 / 2}[\mathrm{~h}]$ & $1 / \mathrm{T}\left[\mathrm{K}^{-1}\right]$ & $\operatorname{In} k$ \\
\hline $20^{\circ} \mathrm{C}$ & 164,6 & $3,41 \mathrm{E}-03$ & $-13,66$ \\
$30^{\circ} \mathrm{C}$ & 39,9 & $3,30 \mathrm{E}-03$ & $-12,24$ \\
$40^{\circ} \mathrm{C}$ & 12,4 & $3,19 \mathrm{E}-03$ & $-11,07$ \\
$50^{\circ} \mathrm{C}$ & 6,2 & $3,10 \mathrm{E}-03$ & $-10,37$ \\
$60^{\circ} \mathrm{C}$ & 2,8 & $3,00 \mathrm{E}-03$ & $-9,59$
\end{tabular}

Commercially obtained "anhydrous" toluene- $\mathrm{d}_{8}$ was used. Karl Fischer titration led to a moisture content of 104 ppm. 


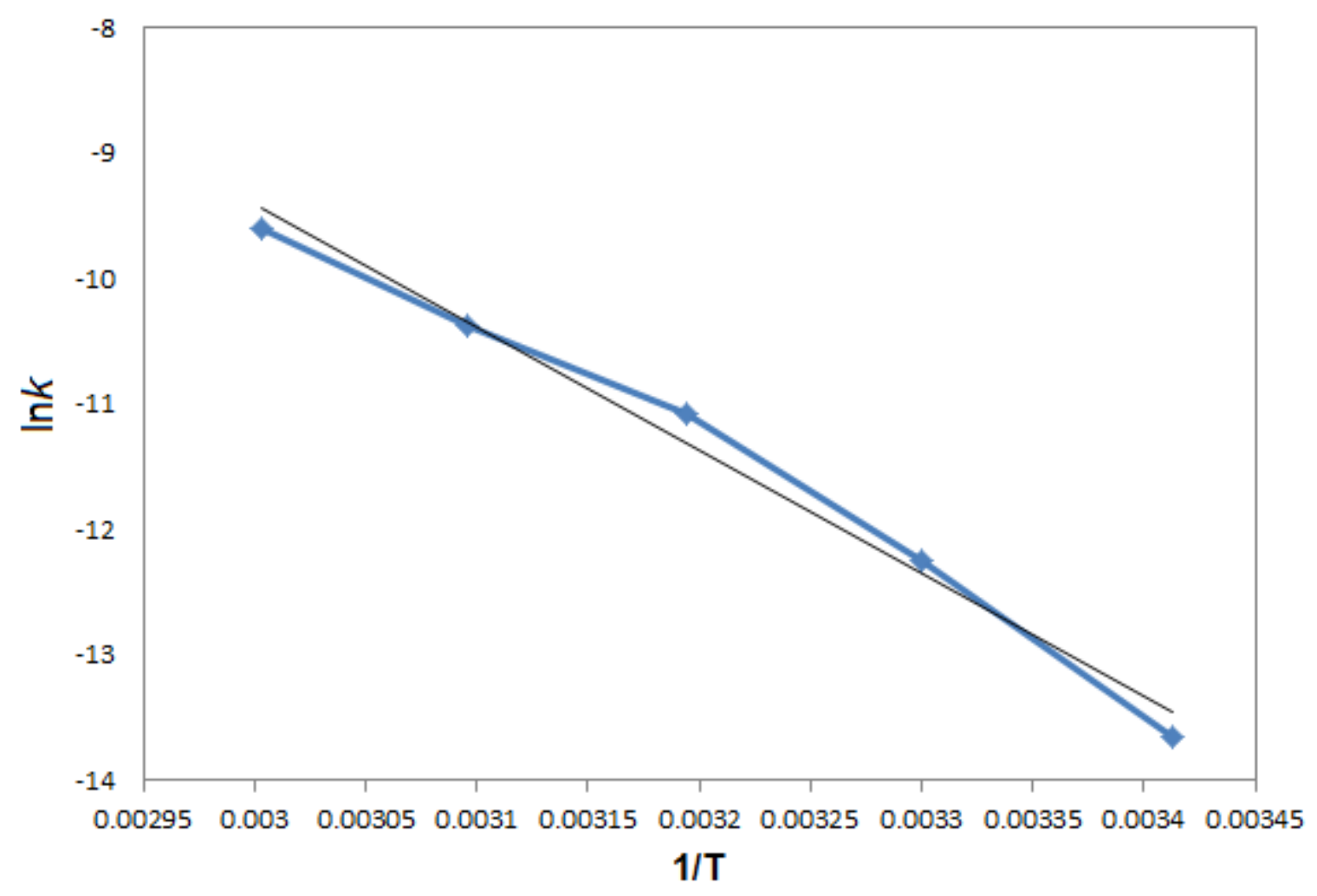

Figure S-81: Arrhenius-plot for the elimination reaction of 8.

Table S- 2: Conversion of the elimination reaction of $26 \mathbf{b}$ with alkali salts $(20 \mathrm{~mol} \%)$ in toluene- $\mathrm{d}_{8}$ at $40^{\circ} \mathrm{C}\left(\mathrm{Ar}^{\mathrm{F}}=\right.$ pentafluorophenyl).

\begin{tabular}{|c|c|c|c|c|c|c|c|}
\hline \multicolumn{2}{|c|}{ Lil } & \multicolumn{2}{|c|}{$\mathrm{Li}_{2} \mathrm{CO}_{3}$} & \multicolumn{2}{|c|}{$\mathrm{LiB}(\operatorname{Ar})_{4}$} & \multicolumn{2}{|c|}{$\mathrm{KB}\left(\operatorname{Ar}^{\mathrm{F}}\right)_{4}$} \\
\hline$T(h)$ & $X_{i}(\%)$ & $T(h)$ & $X_{i}(\%)$ & $T(h)$ & $X_{i}(\%)$ & $T(h)$ & $X_{i}(\%)$ \\
\hline 57 & 16 & 57 & 2 & 1 & 28 & 1 & 5 \\
\hline 81 & 20 & 81 & 2 & 2 & 45 & 2 & 8 \\
\hline 153 & 29 & 153 & 3 & 3 & 56 & 3 & 17 \\
\hline 225 & 36 & 225 & 3 & 4 & 68 & 4 & 23 \\
\hline 297 & 42 & 297 & 3 & 5 & 77 & 5 & 30 \\
\hline 537 & 62 & 537 & 3 & 6 & 84 & 6 & 37 \\
\hline & & & & 7 & 89 & 7 & 44 \\
\hline & & & & 8 & 92 & 8 & 51 \\
\hline
\end{tabular}




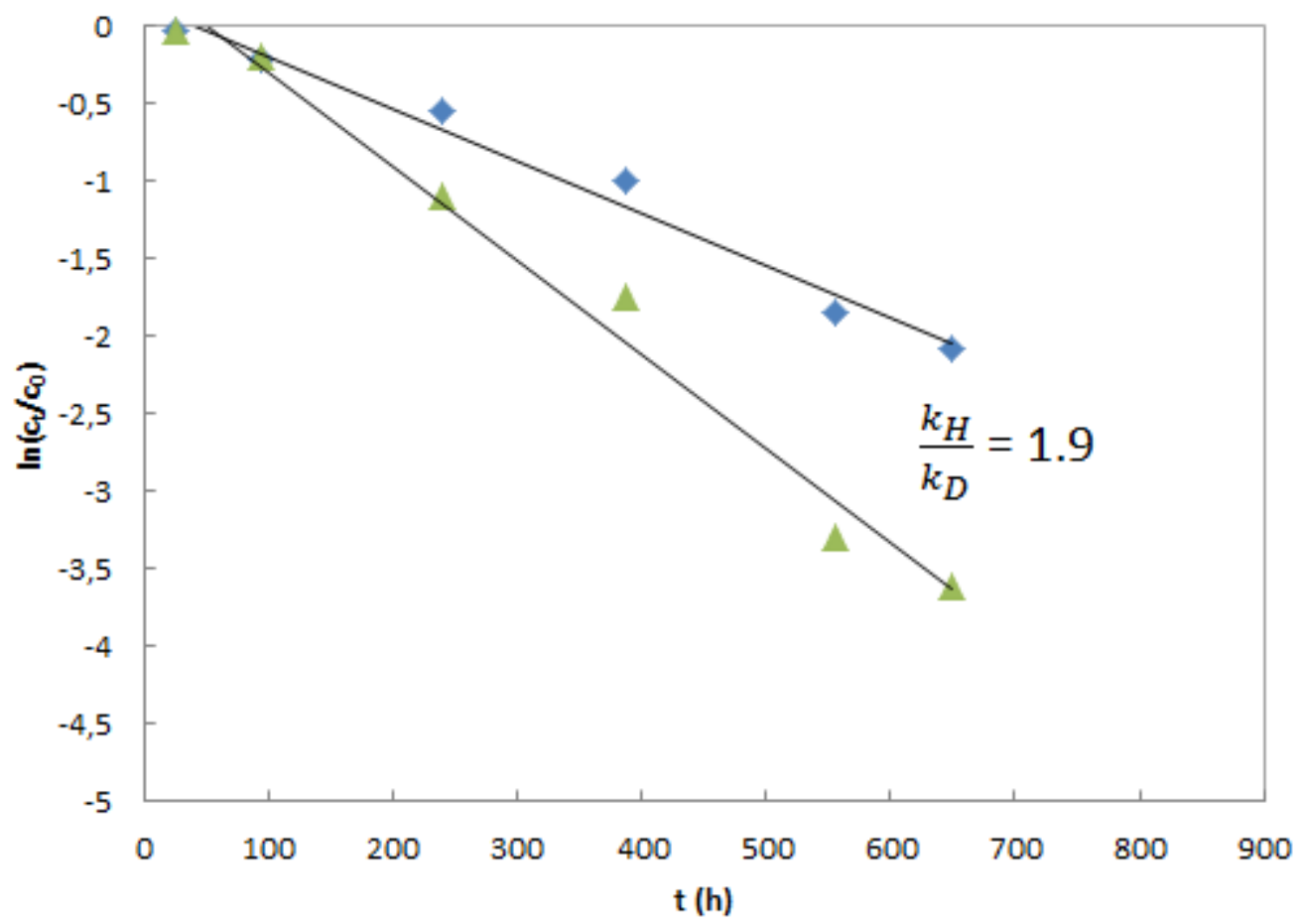

Figure S-82: Isotope effect of the decay of amine 8 with $\mathrm{H}_{2} \mathrm{O} / \mathrm{D}_{2} \mathrm{O}$ in toluene-d8. 


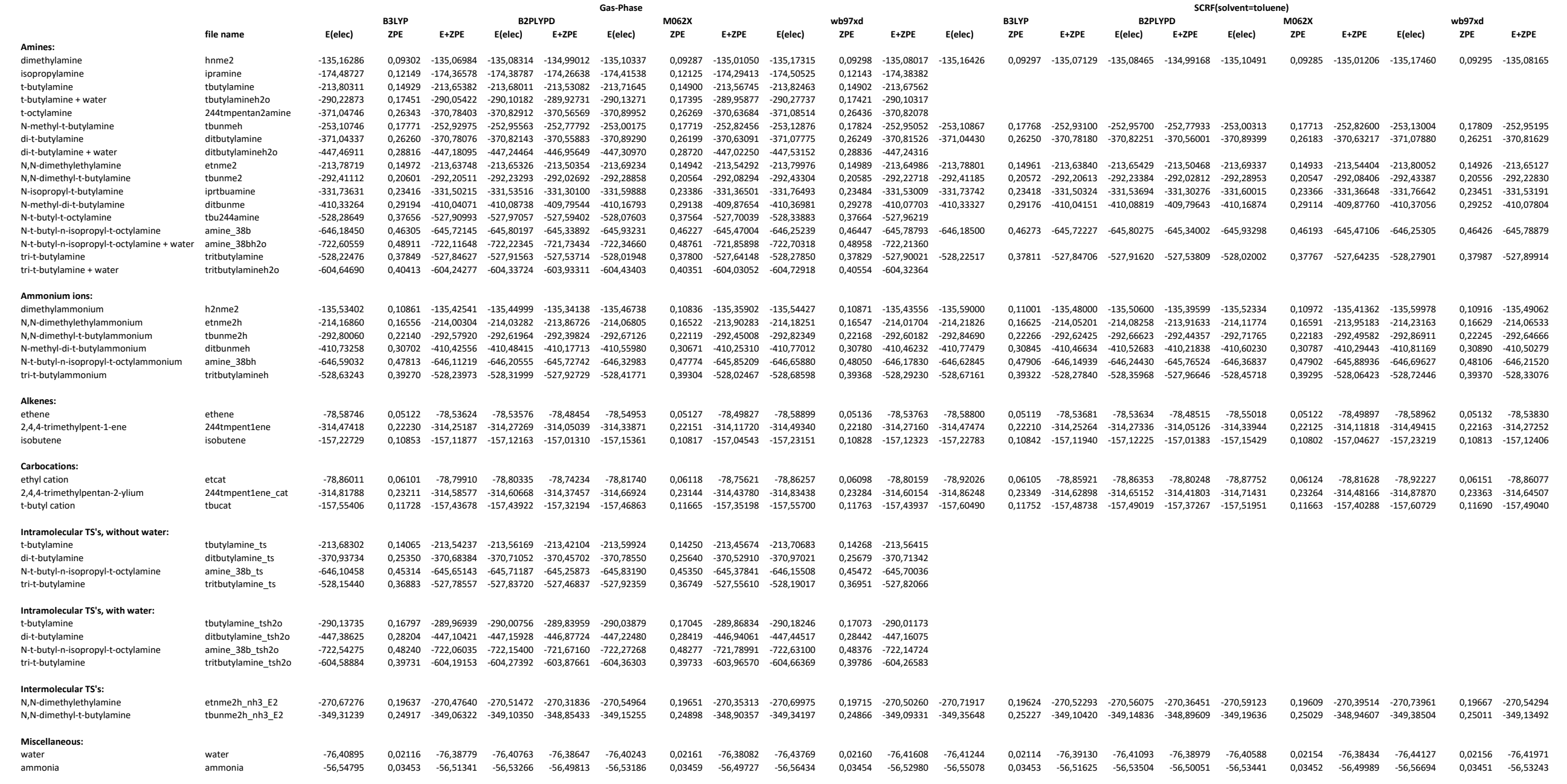


Dimethylamine

Full file name: hnme2.log

Method: B3LYP/6-31G(d) opt/freq

Point group: CS

NImag: 0

E (electronic): $\quad-135.162855424$

Zero-point correction=

Thermal correction to Energy=

0.093018 (Hartree/Particle)

Thermal correction to Enthalpy=

0.097378

Thermal correction to Gibbs Free Energy=

Sum of electronic and zero-point Energies=

0.098322

Sum of electronic and thermal Energies=

0.067593

$-135.069837$

$-135.065478$

Standard orientation:

\begin{tabular}{|c|c|c|c|c|c|}
\hline \multirow{2}{*}{$\begin{array}{l}\text { Center } \\
\text { Number }\end{array}$} & \multirow{2}{*}{$\begin{array}{l}\text { Atomic } \\
\text { Number }\end{array}$} & \multirow{2}{*}{$\begin{array}{c}\text { Atomic } \\
\text { Type }\end{array}$} & \multicolumn{3}{|c|}{ Coordinates (Angstroms) } \\
\hline & & & $\mathrm{X}$ & Y & Z \\
\hline 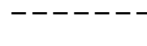 & & & 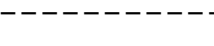 & ---1 & ----- \\
\hline 1 & 7 & 0 & -0.027084 & 0.584827 & 0.000000 \\
\hline 2 & 1 & 0 & 0.794375 & 1.185909 & 0.000000 \\
\hline 3 & 6 & 0 & -0.027084 & -0.222130 & 1.213320 \\
\hline 4 & 6 & 0 & -0.027084 & -0.222130 & -1.213320 \\
\hline 5 & 1 & 0 & 0.032570 & 0.431447 & 2.090116 \\
\hline 6 & 1 & 0 & -0.969807 & -0.779376 & 1.277125 \\
\hline 7 & 1 & 0 & 0.797344 & -0.959137 & 1.273148 \\
\hline 8 & 1 & 0 & 0.032570 & 0.431447 & -2.090116 \\
\hline 9 & 1 & 0 & -0.969807 & -0.779376 & -1.277125 \\
\hline 10 & 1 & 0 & 0.797344 & -0.959137 & -1.273148 \\
\hline
\end{tabular}

Isopropylamine, CS conformation

Full file name: ipramine.log

Method: B3LYP/6-31G(d) opt/freq

Point group: CS

NImag: $\quad 0$

E(electronic): $\quad-174.487273877$

Zero-point correction=

Thermal correction to Energy=

Thermal correction to Enthalpy=

Thermal correction to Gibbs Free Energy=

Sum of electronic and zero-point Energies=

Sum of electronic and thermal Energies=

$0.121490 \quad$ (Hartree/Particle)
0.127003
0.127948
0.094060
$\quad-174.365784$
$\quad-174.360270$

Standard orientation:

$\begin{array}{llll}\text { Center } & \text { Atomic } & \text { Atomic } & \text { Coordinates (Angstroms) } \\ \text { Number } & \text { Number } & \text { Type } & \mathrm{X}\end{array}$

$\begin{array}{llllll}1 & 7 & 0 & -0.916821 & 1.057632 & 0.000000\end{array}$

\section{S-86}




\begin{tabular}{|c|c|c|c|c|c|}
\hline 2 & 6 & 0 & 0.292501 & 0.218098 & 0.000000 \\
\hline 3 & 1 & 0 & 1.222578 & 0.818546 & 0.000000 \\
\hline 4 & 6 & 0 & 0.292501 & -0.642883 & 1.265451 \\
\hline 5 & 6 & 0 & 0.292501 & -0.642883 & -1.265451 \\
\hline 6 & 1 & 0 & -0.906339 & 1.671795 & 0.814136 \\
\hline 7 & 1 & 0 & -0.906339 & 1.671795 & -0.814136 \\
\hline 8 & 1 & 0 & -0.604731 & -1.271092 & 1.295851 \\
\hline 9 & 1 & 0 & 1.176781 & -1.289231 & 1.300253 \\
\hline 10 & 1 & 0 & 0.299363 & -0.019448 & 2.168658 \\
\hline 11 & 1 & 0 & -0.604731 & -1.271092 & -1.295851 \\
\hline 12 & 1 & 0 & 1.176781 & -1.289231 & -1.300253 \\
\hline 13 & 1 & 0 & 0.299363 & -0.019448 & -2.168658 \\
\hline
\end{tabular}

t-Butylamine

Full file name: tbutylamine.log

Method: B3LYP/6-31G(d) opt/freq

Point group: CS

NImag: 0

E(electronic): $\quad-213.803108197$

Zero-point correction=

0.149287 (Hartree/Particle)

Thermal correction to Energy=

0.156048

Thermal correction to Enthalpy=

0.156993

Thermal correction to Gibbs Free Energy=

Sum of electronic and zero-point Energies=

0.120312

$-213.653821$

Sum of electronic and thermal Energies=

$-213.647060$

Standard orientation:

\begin{tabular}{|c|c|c|c|c|c|}
\hline \multirow{2}{*}{$\begin{array}{l}\text { Center } \\
\text { Number }\end{array}$} & \multirow{2}{*}{$\begin{array}{l}\text { Atomic } \\
\text { Number }\end{array}$} & \multirow{2}{*}{$\begin{array}{c}\text { Atomic } \\
\text { Type }\end{array}$} & \multicolumn{3}{|c|}{ Coordinates (Angstroms) } \\
\hline & & & $\mathrm{X}$ & $\mathrm{Y}$ & Z \\
\hline 1 & 7 & 0 & -0.548845 & 1.389611 & 0.000000 \\
\hline 2 & 6 & 0 & -0.011389 & 0.011815 & -0.000000 \\
\hline 3 & 6 & 0 & 1.531839 & -0.018898 & 0.000000 \\
\hline 4 & 6 & 0 & -0.548845 & -0.686571 & 1.258834 \\
\hline 5 & 6 & 0 & -0.548845 & -0.686571 & -1.258834 \\
\hline 6 & 1 & 0 & -0.187238 & 1.890126 & 0.813171 \\
\hline 7 & 1 & 0 & -0.187238 & 1.890126 & -0.813171 \\
\hline 8 & 1 & 0 & 1.920881 & -1.045015 & 0.000000 \\
\hline 9 & 1 & 0 & 1.931384 & 0.489153 & -0.887113 \\
\hline 10 & 1 & 0 & 1.931384 & 0.489153 & 0.887113 \\
\hline 11 & 1 & 0 & -1.643038 & -0.659309 & 1.271632 \\
\hline 12 & 1 & 0 & -0.221907 & -1.731772 & 1.299997 \\
\hline 13 & 1 & 0 & -0.186962 & -0.188653 & 2.167927 \\
\hline 14 & 1 & 0 & -0.221907 & -1.731772 & -1.299997 \\
\hline 15 & 1 & 0 & -1.643038 & -0.659309 & -1.271632 \\
\hline 16 & 1 & 0 & -0.186962 & -0.188653 & -2.167927 \\
\hline
\end{tabular}


t-Butylamine, H-bonded to one water molecule

Full file name: tbutylamineh2o.log

Method: B3LYP/6-31G(d) opt/freq

Point group: $\quad \mathrm{Cl}$

NImag: $\quad 0$

E (electronic) : $\quad-290.228728013$

Zero-point correction=

Thermal correction to Energy=

0.174509 (Hartree/Particle)

Thermal correction to Enthalpy=

0.184158

Thermal correction to Gibbs Free Energy=

Sum of electronic and zero-point Energies=

0.185102

Sum of electronic and thermal Energies=

0.141267

$-290.054219$

$-290.044570$

Standard orientation:

\begin{tabular}{|c|c|c|c|c|c|}
\hline \multirow{2}{*}{$\begin{array}{l}\text { Center } \\
\text { Number }\end{array}$} & \multirow{2}{*}{$\begin{array}{l}\text { Atomic } \\
\text { Number }\end{array}$} & \multirow{2}{*}{$\begin{array}{c}\text { Atomic } \\
\text { Type }\end{array}$} & \multicolumn{3}{|c|}{ Coordinates (Angstroms) } \\
\hline & & & $\mathrm{X}$ & Y & Z \\
\hline & & & & 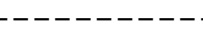 & --- \\
\hline 1 & 7 & 0 & 0.205012 & -0.031799 & 1.203082 \\
\hline 2 & 6 & 0 & -0.653179 & -0.002508 & -0.013375 \\
\hline 3 & 6 & 0 & -2.148984 & -0.050829 & 0.349930 \\
\hline 4 & 6 & 0 & -0.325004 & 1.294046 & -0.769724 \\
\hline 5 & 6 & 0 & -0.265104 & -1.220804 & -0.865698 \\
\hline 6 & 1 & 0 & -0.007993 & 0.776223 & 1.789689 \\
\hline 7 & 1 & 0 & -0.027605 & -0.853557 & 1.762914 \\
\hline 8 & 1 & 0 & -2.779292 & -0.031104 & -0.547260 \\
\hline 9 & 1 & 0 & -2.389636 & -0.966281 & 0.905840 \\
\hline 10 & 1 & 0 & -2.429707 & 0.807534 & 0.973534 \\
\hline 11 & 1 & 0 & 0.740329 & 1.330683 & -1.015519 \\
\hline 12 & 1 & 0 & -0.904274 & 1.356084 & -1.697578 \\
\hline 13 & 1 & 0 & -0.573068 & 2.175660 & -0.164046 \\
\hline 14 & 1 & 0 & -0.833786 & -1.239015 & -1.801793 \\
\hline 15 & 1 & 0 & 0.802697 & -1.180146 & -1.100223 \\
\hline 16 & 1 & 0 & -0.472414 & -2.157987 & -0.331968 \\
\hline 17 & 1 & 0 & 2.001182 & 0.045275 & 0.542709 \\
\hline 18 & 8 & 0 & 2.821606 & 0.102104 & -0.003027 \\
\hline 19 & 1 & 0 & 3.219253 & -0.777033 & 0.079537 \\
\hline
\end{tabular}

2,4,4-Trimethylpentan-2-amine, conformation \#1

Full file name: 244 tmpentan2amine.log

Method: B3LYP/6-31G(d) opt/freq

Point group: $\quad$ C1

NImag: $\quad 0$

E (electronic) : $\quad-371.047462379$

Zero-point correction=

Thermal correction to Energy=

0.263432 (Hartree/Particle)

Thermal correction to Enthalpy=

0.275134

0.276078

Thermal correction to Gibbs Free Energy=

Sum of electronic and zero-point Energies=

0.227794

Sum of electronic and thermal Energies=

$-370.784031$

$-370.772328$ 
Standard orientation:

\begin{tabular}{|c|c|c|c|c|c|}
\hline \multirow{2}{*}{$\begin{array}{l}\text { Center } \\
\text { Number }\end{array}$} & \multirow{2}{*}{$\begin{array}{l}\text { Atomic } \\
\text { Number }\end{array}$} & \multirow{2}{*}{$\begin{array}{c}\text { Atomic } \\
\text { Type }\end{array}$} & \multicolumn{3}{|c|}{ Coordinates (Angstroms) } \\
\hline & & & $\mathrm{X}$ & Y & Z \\
\hline 1 & 7 & 0 & 1.344599 & -0.530355 & 1.339484 \\
\hline 2 & 6 & 0 & 1.371912 & -0.028610 & -0.051433 \\
\hline 3 & 6 & 0 & 0.000769 & -0.270411 & -0.752117 \\
\hline 4 & 6 & 0 & -1.376752 & -0.015282 & -0.064732 \\
\hline 5 & 6 & 0 & -2.439244 & -0.098074 & -1.184100 \\
\hline 6 & 6 & 0 & 2.447169 & -0.757904 & -0.894111 \\
\hline 7 & 6 & 0 & 1.751255 & 1.462890 & 0.006762 \\
\hline 8 & 6 & 0 & -1.698335 & -1.104470 & 0.980307 \\
\hline 9 & 6 & 0 & -1.484725 & 1.365055 & 0.608453 \\
\hline 10 & 1 & 0 & 2.267710 & -0.392516 & 1.754232 \\
\hline 11 & 1 & 0 & 1.197969 & -1.540510 & 1.330116 \\
\hline 12 & 1 & 0 & 0.029054 & 0.319604 & -1.679181 \\
\hline 13 & 1 & 0 & -0.016056 & -1.320217 & -1.082748 \\
\hline 14 & 1 & 0 & -3.449376 & 0.009559 & -0.770339 \\
\hline 15 & 1 & 0 & -2.297131 & 0.693836 & -1.930278 \\
\hline 16 & 1 & 0 & -2.394849 & -1.061885 & -1.707200 \\
\hline 17 & 1 & 0 & 2.463780 & -0.404561 & -1.932981 \\
\hline 18 & 1 & 0 & 3.446295 & -0.593626 & -0.470503 \\
\hline 19 & 1 & 0 & 2.261759 & -1.839295 & -0.913129 \\
\hline 20 & 1 & 0 & 1.751777 & 1.904309 & -0.996147 \\
\hline 21 & 1 & 0 & 1.069552 & 2.034111 & 0.637815 \\
\hline 22 & 1 & 0 & 2.763168 & 1.578917 & 0.417218 \\
\hline 23 & 1 & 0 & -1.637351 & -2.107914 & 0.537864 \\
\hline 24 & 1 & 0 & -1.009302 & -1.053912 & 1.826304 \\
\hline 25 & 1 & 0 & -2.719408 & -0.976895 & 1.361857 \\
\hline 26 & 1 & 0 & -1.238503 & 2.176581 & -0.087335 \\
\hline 27 & 1 & 0 & -2.511460 & 1.532725 & 0.958298 \\
\hline 28 & 1 & 0 & -0.822103 & 1.435008 & 1.475585 \\
\hline
\end{tabular}

t-Butylmethylamine

Full file name: tbunmeh.log

Method: B3LYP/6-31G(d) opt/freq

Point group: $\quad \mathrm{C} 1$

NImag: $\quad 0$

E (electronic): $\quad-253.107456962$

Zero-point correction=

Thermal correction to Energy=

0.177711 (Hartree/Particle)

Thermal correction to Enthalpy=

0.185825

Thermal correction to Gibbs Free Energy=

0.186769

Sum of electronic and zero-point Energies=

0.146529

Sum of electronic and thermal Energies=

$-252.929746$

$-252.921632$

Standard orientation: 


\begin{tabular}{|c|c|c|c|c|c|}
\hline \multirow{2}{*}{$\begin{array}{l}\text { Center } \\
\text { Number }\end{array}$} & \multirow{2}{*}{$\begin{array}{l}\text { Atomic } \\
\text { Number }\end{array}$} & \multirow{2}{*}{$\begin{array}{c}\text { Atomic } \\
\text { Type }\end{array}$} & \multicolumn{3}{|c|}{ Coordinates (Angstroms) } \\
\hline & & & $\mathrm{x}$ & $\mathrm{Y}$ & $\mathrm{z}$ \\
\hline 1 & 7 & 0 & -0.831438 & -0.448720 & -0.670059 \\
\hline 2 & 6 & 0 & 0.409279 & 0.004847 & -0.001658 \\
\hline 3 & 6 & 0 & 0.472791 & 1.541467 & -0.045394 \\
\hline 4 & 6 & 0 & 0.529154 & -0.484749 & 1.459698 \\
\hline 5 & 6 & 0 & 1.571399 & -0.572072 & -0.828028 \\
\hline 6 & 6 & 0 & -2.106290 & -0.051188 & -0.079250 \\
\hline 7 & 1 & 0 & -0.802586 & -1.465746 & -0.727652 \\
\hline 8 & 1 & 0 & 1.444473 & 1.893175 & 0.319009 \\
\hline 9 & 1 & 0 & 0.335990 & 1.898009 & -1.071835 \\
\hline 10 & 1 & 0 & -0.297817 & 2.001521 & 0.583042 \\
\hline 11 & 1 & 0 & 0.493637 & -1.580801 & 1.507091 \\
\hline 12 & 1 & 0 & 1.476896 & -0.162659 & 1.907679 \\
\hline 13 & 1 & 0 & -0.279558 & -0.093364 & 2.086678 \\
\hline 14 & 1 & 0 & 1.546658 & -1.669958 & -0.826206 \\
\hline 15 & 1 & 0 & 2.538939 & -0.262338 & -0.417421 \\
\hline 16 & 1 & 0 & 1.504869 & -0.234280 & -1.867227 \\
\hline 17 & 1 & 0 & -2.240200 & 1.031932 & -0.166296 \\
\hline 18 & 1 & 0 & -2.913093 & -0.522203 & -0.651096 \\
\hline 19 & 1 & 0 & -2.246133 & -0.322075 & 0.982439 \\
\hline
\end{tabular}

Di-t-butylamine

Full file name: ditbutylamine.log

Method: B3LYP/6-31G(d) opt/freq

Point group: $\quad \mathrm{C} 1$

NImag: $\quad 0$

E(electronic): $\quad-371.043367700$

Zero-point correction=

Thermal correction to Energy=

0.262603 (Hartree/Particle)

Thermal correction to Enthalpy=

0.274365

0.275309

Thermal correction to Gibbs Free Energy=

Sum of electronic and zero-point Energies=

0.227173

$-370.780765$

Sum of electronic and thermal Energies=

$-370.769002$

Standard orientation:

\begin{tabular}{|c|c|c|c|c|c|}
\hline \multirow{2}{*}{$\begin{array}{l}\text { Center } \\
\text { Number }\end{array}$} & \multirow{2}{*}{$\begin{array}{l}\text { Atomic } \\
\text { Number }\end{array}$} & \multirow{2}{*}{$\begin{array}{l}\text { Atomic } \\
\text { Type }\end{array}$} & \multicolumn{3}{|c|}{ Coordinates (Angstroms) } \\
\hline & & & $\mathrm{x}$ & $\mathrm{Y}$ & Z \\
\hline 1 & 7 & 0 & -0.003998 & -0.037596 & 0.721302 \\
\hline 2 & 6 & 0 & -1.331254 & -0.000298 & 0.060423 \\
\hline 3 & 6 & 0 & 1.326092 & -0.007253 & 0.062390 \\
\hline 4 & 6 & 0 & -1.722568 & -1.324044 & -0.643700 \\
\hline 5 & 6 & 0 & -2.332545 & 0.241566 & 1.210792 \\
\hline 6 & 6 & 0 & -1.451126 & 1.165256 & -0.933930 \\
\hline 7 & 6 & 0 & 1.487821 & -0.905242 & -1.186303 \\
\hline 8 & 6 & 0 & 1.715576 & 1.443274 & -0.294785 \\
\hline 9 & 6 & 0 & 2.310402 & -0.502336 & 1.142398 \\
\hline
\end{tabular}




$\begin{array}{rrrrrr}10 & 1 & 0 & -0.018910 & -0.827915 & 1.362175 \\ 11 & 1 & 0 & -2.766425 & -1.291419 & -0.980277 \\ 12 & 1 & 0 & -1.099438 & -1.529586 & -1.517521 \\ 13 & 1 & 0 & -1.625587 & -2.171174 & 0.047442 \\ 14 & 1 & 0 & -2.105970 & 1.182612 & 1.721265 \\ 15 & 1 & 0 & -3.362478 & 0.278818 & 0.836909 \\ 16 & 1 & 0 & -2.278145 & -0.565615 & 1.952925 \\ 17 & 1 & 0 & -2.487774 & 1.245258 & -1.280597 \\ 18 & 1 & 0 & -1.175999 & 2.113035 & -0.460841 \\ 19 & 1 & 0 & -0.822976 & 1.023540 & -1.818984 \\ 20 & 1 & 0 & 0.857536 & -0.571548 & -2.016646 \\ 21 & 1 & 0 & 2.526963 & -0.881928 & -1.537774 \\ 22 & 1 & 0 & 1.234465 & -1.947431 & -0.961299 \\ 23 & 1 & 0 & 1.114547 & 1.846849 & -1.113190 \\ 24 & 1 & 0 & 1.583315 & 2.091700 & 0.577158 \\ 25 & 1 & 0 & 2.766005 & 1.487982 & -0.607084 \\ 26 & 1 & 0 & 3.344332 & -0.437019 & 0.787654 \\ 27 & 1 & 0 & 2.216052 & 0.101966 & 2.051110 \\ 28 & 1 & 0 & 2.114084 & -1.550495 & 1.404744 \\ ----------------1\end{array}$
1
7
$-0.000001203$
0.000003088
0.000001164

Full file name: ditbutylamineh2o.log

Method: B3LYP/6-31G(d) opt/freq

Point group: $\quad$ C1

NImag: 0

E(electronic): $\quad-447.469109149$

Zero-point correction=

Thermal correction to Energy=

Thermal correction to Enthalpy=

0.288155 (Hartree/Particle)

Thermal correction to Gibbs Free

Sum of electronic and zero-point Energies=

0.302759

0.303703

Sum of electronic and thermal Energies=

0.248505

$-447.180954$

$-447.166351$

Standard orientation:

$\begin{array}{llcl}\text { Center } & \text { Atomic } & \text { Atomic } & \text { Coordinates (Angstroms) } \\ \text { Number } & \text { Number } & \text { Type } & \mathrm{X}\end{array}$

$\begin{array}{rrrrrr}1 & 7 & 0 & 0.008716 & 0.128488 & -0.501217 \\ 2 & 6 & 0 & 1.366291 & -0.290996 & -0.029534 \\ 3 & 6 & 0 & -1.312502 & -0.407509 & -0.039154 \\ 4 & 6 & 0 & 1.782155 & -1.703853 & -0.500309 \\ 5 & 6 & 0 & 2.329944 & 0.731214 & -0.672587 \\ 6 & 6 & 0 & 1.496653 & -0.186822 & 1.497047 \\ 7 & 6 & 0 & -1.417520 & -1.946073 & 0.006330 \\ 8 & 6 & 0 & -1.702767 & 0.181119 & 1.332270 \\ 9 & 6 & 0 & -2.319613 & 0.128937 & -1.078584 \\ 10 & 1 & 0 & 0.025293 & 0.028093 & -1.516103 \\ 11 & 1 & 0 & 2.840958 & -1.879521 & -0.275250 \\ 12 & 1 & 0 & 1.206915 & -2.496297 & -0.016069 \\ 13 & 1 & 0 & 1.655555 & -1.806622 & -1.585655\end{array}$




\begin{tabular}{|c|c|c|c|c|c|}
\hline 14 & 1 & 0 & 2.079111 & 1.746894 & -0.353101 \\
\hline 15 & 1 & 0 & 3.366764 & 0.515369 & -0.391656 \\
\hline 16 & 1 & 0 & 2.269885 & 0.690115 & -1.768646 \\
\hline 17 & 1 & 0 & 2.546498 & -0.321603 & 1.781038 \\
\hline 18 & 1 & 0 & 1.171290 & 0.795746 & 1.851890 \\
\hline 19 & 1 & 0 & 0.918903 & -0.958166 & 2.015630 \\
\hline 20 & 1 & 0 & -0.792478 & -2.375135 & 0.795425 \\
\hline 21 & 1 & 0 & -2.452343 & -2.243896 & 0.214177 \\
\hline 22 & 1 & 0 & -1.125458 & -2.397995 & -0.948638 \\
\hline 23 & 1 & 0 & -1.091886 & -0.214770 & 2.146660 \\
\hline 24 & 1 & 0 & -1.609299 & 1.270795 & 1.324287 \\
\hline 25 & 1 & 0 & -2.745353 & -0.075741 & 1.554217 \\
\hline 26 & 1 & 0 & -3.340996 & -0.160030 & -0.810737 \\
\hline 27 & 1 & 0 & -2.269964 & 1.221995 & -1.123184 \\
\hline 28 & 1 & 0 & -2.112861 & -0.272208 & -2.080074 \\
\hline 29 & 1 & 0 & -0.116292 & 1.996087 & -0.188358 \\
\hline 30 & 8 & 0 & -0.200137 & 2.947133 & 0.070301 \\
\hline 31 & 1 & 0 & -0.219993 & 3.424293 & -0.772622 \\
\hline
\end{tabular}

Ethyl dimethyl amine

Full file name: etnme2.log

Method: B3LYP/6-31G(d) opt/freq

Point group: $\quad$ CS

NImag: $\quad 0$

E(electronic): $\quad-213.787194913$

Zero-point correction=

Thermal correction to Energy=

0.149717 (Hartree/Particle)

Thermal correction to Enthalpy=

0.156481

0.157426

Thermal correction to Gibbs Free Energy=

Sum of electronic and zero-point Energies=

0.120174

$-213.637478$

Sum of electronic and thermal Energies=

$-213.630714$

Standard orientation:

\begin{tabular}{|c|c|c|c|c|c|}
\hline \multirow{2}{*}{$\begin{array}{l}\text { Center } \\
\text { Number }\end{array}$} & \multirow{2}{*}{$\begin{array}{l}\text { Atomic } \\
\text { Number }\end{array}$} & \multirow{2}{*}{$\begin{array}{l}\text { Atomic } \\
\text { Type }\end{array}$} & \multicolumn{3}{|c|}{ Coordinates (Angstroms) } \\
\hline & & & $\mathrm{X}$ & $\mathrm{Y}$ & Z \\
\hline 1 & 7 & 0 & -0.654822 & 0.336943 & 0.000000 \\
\hline 2 & 6 & 0 & -0.276596 & -1.076214 & -0.000000 \\
\hline 3 & 6 & 0 & 1.227288 & -1.408220 & -0.000000 \\
\hline 4 & 6 & 0 & -0.276596 & 1.052193 & 1.207525 \\
\hline 5 & 6 & 0 & -0.276596 & 1.052193 & -1.207525 \\
\hline 6 & 1 & 0 & -0.744753 & -1.537850 & 0.879698 \\
\hline 7 & 1 & 0 & -0.744753 & -1.537850 & -0.879698 \\
\hline 8 & 1 & 0 & 1.370811 & -2.495193 & -0.000000 \\
\hline 9 & 1 & 0 & 1.732974 & -1.008024 & 0.885931 \\
\hline 10 & 1 & 0 & 1.732974 & -1.008024 & -0.885931 \\
\hline 11 & 1 & 0 & -0.629471 & 0.501611 & 2.087207 \\
\hline 12 & 1 & 0 & 0.812653 & 1.214936 & 1.321560 \\
\hline 13 & 1 & 0 & -0.757434 & 2.037768 & 1.211583 \\
\hline 14 & 1 & 0 & -0.629471 & 0.501611 & -2.087207 \\
\hline
\end{tabular}

\section{S-92}




\begin{tabular}{|c|c|c|c|c|c|}
\hline 15 & 1 & 0 & 0.812653 & 1.214936 & -1.321560 \\
\hline 16 & 1 & 0 & -0.757434 & 2.037768 & -1.211583 \\
\hline
\end{tabular}

t-Butyldimethylamine

Full file name: tbunme2.log

Method: B3LYP/6-31G(d) opt/freq

Point group: $\quad$ CS

NImag: $\quad 0$

E(electronic): $\quad-292.411121428$

Zero-point correction=

Thermal correction to Energy=

0.206010 (Hartree/Particle)

Thermal correction to Enthalpy=

0.215354

Thermal correction to Gibbs Free Energy=

0.216298

Sum of electronic and zero-point Energies=

0.172963

Sum of electronic and thermal Energies=

$-292.205111$

$-292.195768$

Standard orientation:

\begin{tabular}{|c|c|c|c|c|c|}
\hline \multirow{2}{*}{$\begin{array}{l}\text { Center } \\
\text { Number }\end{array}$} & \multirow{2}{*}{$\begin{array}{l}\text { Atomic } \\
\text { Number }\end{array}$} & \multirow{2}{*}{$\begin{array}{c}\text { Atomic } \\
\text { Type }\end{array}$} & \multicolumn{3}{|c|}{ Coordinates (Angstroms) } \\
\hline & & & $\mathrm{x}$ & $\mathrm{Y}$ & Z \\
\hline 1 & 7 & 0 & -0.562724 & 0.731876 & -0.000000 \\
\hline 2 & 6 & 0 & 0.114342 & -0.598414 & 0.000000 \\
\hline 3 & 6 & 0 & 1.658538 & -0.501754 & 0.000000 \\
\hline 4 & 6 & 0 & -0.344557 & -1.389497 & 1.242647 \\
\hline 5 & 6 & 0 & -0.344557 & -1.389497 & -1.242647 \\
\hline 6 & 6 & 0 & -0.344557 & 1.544682 & 1.191926 \\
\hline 7 & 6 & 0 & -0.344557 & 1.544682 & -1.191926 \\
\hline 8 & 1 & 0 & 2.105727 & -1.502672 & 0.000000 \\
\hline 9 & 1 & 0 & 2.030159 & 0.024246 & -0.886502 \\
\hline 10 & 1 & 0 & 2.030159 & 0.024246 & 0.886502 \\
\hline 11 & 1 & 0 & -1.437457 & -1.381730 & 1.317141 \\
\hline 12 & 1 & 0 & -0.010589 & -2.429790 & 1.166369 \\
\hline 13 & 1 & 0 & 0.068560 & -0.987706 & 2.173116 \\
\hline 14 & 1 & 0 & -1.437457 & -1.381730 & -1.317141 \\
\hline 15 & 1 & 0 & -0.010589 & -2.429790 & -1.166369 \\
\hline 16 & 1 & 0 & 0.068560 & -0.987706 & -2.173116 \\
\hline 17 & 1 & 0 & 0.697048 & 1.897494 & 1.319368 \\
\hline 18 & 1 & 0 & -0.982253 & 2.433750 & 1.129127 \\
\hline 19 & 1 & 0 & -0.632754 & 1.002896 & 2.095192 \\
\hline 20 & 1 & 0 & 0.697048 & 1.897494 & -1.319368 \\
\hline 21 & 1 & 0 & -0.982253 & 2.433750 & -1.129127 \\
\hline 22 & 1 & 0 & -0.632754 & 1.002896 & -2.095192 \\
\hline
\end{tabular}

Isopropyl t-butyl amine, conformation \#1

Full file name: iprtbuamine.log

Method: B3LYP/6-31G(d) opt/freq 


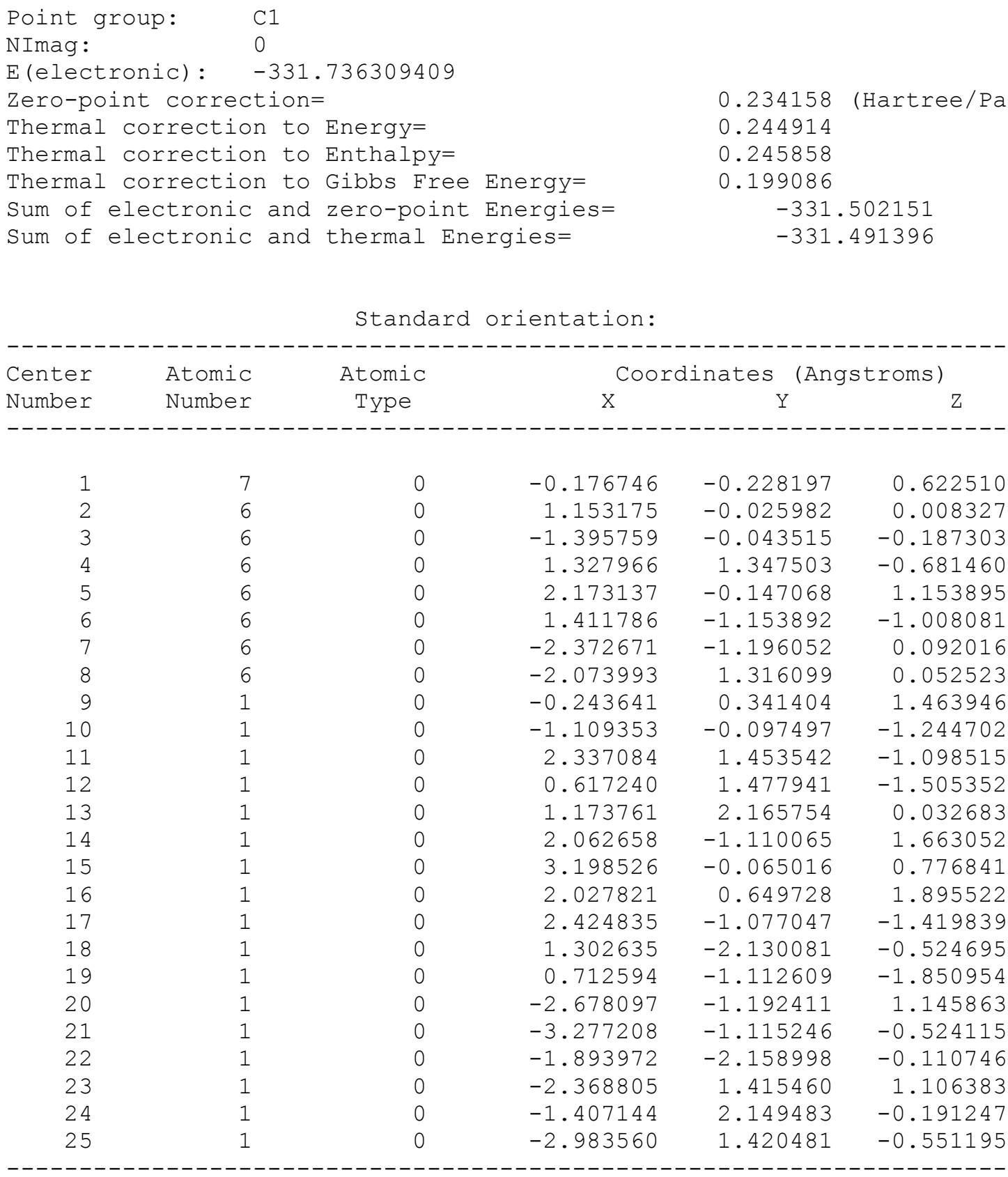

Di-t-butylmethylamine

Full file name: ditbunme.log

Method: $\quad$ B3LYP/6-31G(d) opt/freq

Point group: $\quad \mathrm{C} 1$

NImag: $\quad 0$

E (electronic): $\quad-410.332643156$

Zero-point correction=

Thermal correction to Energy=

Thermal correction to Enthalpy=

0.291938 (Hartree/Particle)

Thermal correction to Gibbs Free Energy=

0.304637

0.305581

0.255675 
Sum of electronic and zero-point Energies= Sum of electronic and thermal Energies=
$-410.040705$

$-410.028006$

Standard orientation:

\begin{tabular}{|c|c|c|c|c|c|}
\hline \multirow{2}{*}{$\begin{array}{l}\text { Center } \\
\text { Number }\end{array}$} & \multirow{2}{*}{$\begin{array}{l}\text { Atomic } \\
\text { Number }\end{array}$} & \multirow{2}{*}{$\begin{array}{c}\text { Atomic } \\
\text { Type }\end{array}$} & \multicolumn{3}{|c|}{ Coordinates (Angstroms) } \\
\hline & & & $\mathrm{x}$ & $\mathrm{Y}$ & Z \\
\hline 1 & 7 & 0 & 0.006507 & 0.485629 & -0.377923 \\
\hline 2 & 6 & 0 & -0.001968 & 1.932768 & -0.140275 \\
\hline 3 & 6 & 0 & -1.323309 & -0.131102 & -0.027420 \\
\hline 4 & 6 & 0 & 1.321397 & -0.151088 & -0.018889 \\
\hline 5 & 6 & 0 & -1.842268 & 0.267562 & 1.380002 \\
\hline 6 & 6 & 0 & -2.347039 & 0.340830 & -1.092968 \\
\hline 7 & 6 & 0 & -1.344038 & -1.670027 & -0.104493 \\
\hline 8 & 6 & 0 & 1.491637 & -0.447047 & 1.491683 \\
\hline 9 & 6 & 0 & 2.468441 & 0.808574 & -0.429612 \\
\hline 10 & 6 & 0 & 1.575694 & -1.439198 & -0.841954 \\
\hline 11 & 1 & 0 & 0.677773 & 2.445288 & -0.824875 \\
\hline 12 & 1 & 0 & -0.993857 & 2.338699 & -0.335288 \\
\hline 13 & 1 & 0 & 0.269191 & 2.230261 & 0.888760 \\
\hline 14 & 1 & 0 & -2.838648 & -0.158284 & 1.547846 \\
\hline 15 & 1 & 0 & -1.185528 & -0.102852 & 2.172855 \\
\hline 16 & 1 & 0 & -1.930237 & 1.352108 & 1.495886 \\
\hline 17 & 1 & 0 & -3.313824 & -0.149458 & -0.930205 \\
\hline 18 & 1 & 0 & -1.987967 & 0.081154 & -2.093803 \\
\hline 19 & 1 & 0 & -2.529873 & 1.418984 & -1.069711 \\
\hline 20 & 1 & 0 & -2.370758 & -2.006554 & 0.075894 \\
\hline 21 & 1 & 0 & -1.047788 & -2.031605 & -1.092458 \\
\hline 22 & 1 & 0 & -0.716348 & -2.147357 & 0.651396 \\
\hline 23 & 1 & 0 & 2.508331 & -0.803621 & 1.698176 \\
\hline 24 & 1 & 0 & 0.799137 & -1.216642 & 1.844318 \\
\hline 25 & 1 & 0 & 1.331135 & 0.455452 & 2.092586 \\
\hline 26 & 1 & 0 & 3.424877 & 0.295352 & -0.288989 \\
\hline 27 & 1 & 0 & 2.388570 & 1.087788 & -1.486012 \\
\hline 28 & 1 & 0 & 2.501817 & 1.720706 & 0.171948 \\
\hline 29 & 1 & 0 & 2.638925 & -1.700325 & -0.792674 \\
\hline 30 & 1 & 0 & 1.314915 & -1.268821 & -1.891557 \\
\hline 31 & 1 & 0 & 1.023324 & -2.307297 & -0.485075 \\
\hline
\end{tabular}

$\mathrm{N}$-(tert-butyl)-2,4,4-trimethylpentan-2-amine, conformation \#1

Full file name: tbu244amine.log

Method: B3LYP/6-31G(d) opt/freq

Point group: $\quad \mathrm{C} 1$

NImag: $\quad 0$

E (electronic): $\quad-528.286488037$

Zero-point correction=

Thermal correction to Energy=

Thermal correction to Enthalpy=

Thermal correction to Gibbs Free Energy=

Sum of electronic and zero-point Energies=

0.376555 (Hartree/Particle)
0.393406
0.394351
0.334793
$\quad-527.909933$ 
Standard orientation:

\begin{tabular}{|c|c|c|c|c|c|}
\hline \multirow{2}{*}{$\begin{array}{l}\text { Center } \\
\text { Number }\end{array}$} & \multirow{2}{*}{$\begin{array}{l}\text { Atomic } \\
\text { Number }\end{array}$} & \multirow{2}{*}{$\begin{array}{c}\text { Atomic } \\
\text { Type }\end{array}$} & \multicolumn{3}{|c|}{ Coordinates (Angstroms) } \\
\hline & & & $\mathrm{X}$ & Y & Z \\
\hline & & & & & \\
\hline 1 & 7 & 0 & -0.759140 & -0.198291 & -0.368993 \\
\hline 2 & 6 & 0 & -0.040245 & 1.062602 & -0.069899 \\
\hline 3 & 6 & 0 & 1.465867 & 0.858543 & -0.456041 \\
\hline 4 & 6 & 0 & 2.329021 & -0.375256 & -0.041453 \\
\hline 5 & 6 & 0 & 2.214261 & -0.742059 & 1.449053 \\
\hline 6 & 6 & 0 & -0.181455 & 1.481999 & 1.405131 \\
\hline 7 & 6 & 0 & -0.515519 & 2.260641 & -0.941593 \\
\hline 8 & 6 & 0 & 1.992548 & -1.620836 & -0.887791 \\
\hline 9 & 6 & 0 & 3.796857 & 0.012860 & -0.333988 \\
\hline 10 & 6 & 0 & -2.168155 & -0.530576 & -0.027986 \\
\hline 11 & 6 & 0 & -2.276743 & -0.985435 & 1.442722 \\
\hline 12 & 6 & 0 & -3.217411 & 0.573301 & -0.300164 \\
\hline 13 & 6 & 0 & -2.511681 & -1.743461 & -0.918186 \\
\hline 14 & 1 & 0 & -0.630562 & -0.378795 & -1.362146 \\
\hline 15 & 1 & 0 & 1.525959 & 0.912995 & -1.554111 \\
\hline 16 & 1 & 0 & 1.983978 & 1.759858 & -0.099542 \\
\hline 17 & 1 & 0 & 2.936631 & -1.529913 & 1.698345 \\
\hline 18 & 1 & 0 & 2.428026 & 0.116413 & 2.097239 \\
\hline 19 & 1 & 0 & 1.216233 & -1.119151 & 1.689606 \\
\hline 20 & 1 & 0 & 0.481386 & 2.330971 & 1.607253 \\
\hline 21 & 1 & 0 & -1.201114 & 1.811247 & 1.629358 \\
\hline 22 & 1 & 0 & 0.076385 & 0.679700 & 2.096537 \\
\hline 23 & 1 & 0 & 0.159683 & 3.117706 & -0.825851 \\
\hline 24 & 1 & 0 & -0.522558 & 1.988041 & -2.004392 \\
\hline 25 & 1 & 0 & -1.519954 & 2.594550 & -0.671382 \\
\hline 26 & 1 & 0 & 0.975426 & -1.965393 & -0.691220 \\
\hline 27 & 1 & 0 & 2.084490 & -1.405723 & -1.960916 \\
\hline 28 & 1 & 0 & 2.687417 & -2.438544 & -0.657044 \\
\hline 29 & 1 & 0 & 4.120395 & 0.855318 & 0.290226 \\
\hline 30 & 1 & 0 & 4.469748 & -0.830030 & -0.133730 \\
\hline 31 & 1 & 0 & 3.931357 & 0.303861 & -1.383589 \\
\hline 32 & 1 & 0 & -2.118885 & -0.164737 & 2.146287 \\
\hline 33 & 1 & 0 & -3.274869 & -1.395978 & 1.637487 \\
\hline 34 & 1 & 0 & -1.535863 & -1.763628 & 1.652488 \\
\hline 35 & 1 & 0 & -3.082944 & 1.440149 & 0.353981 \\
\hline 36 & 1 & 0 & -3.172615 & 0.917360 & -1.339492 \\
\hline 37 & 1 & 0 & -4.227458 & 0.184783 & -0.119765 \\
\hline 38 & 1 & 0 & -3.515520 & -2.118526 & -0.692884 \\
\hline 39 & 1 & 0 & -2.489529 & -1.472265 & -1.982028 \\
\hline 40 & 1 & 0 & -1.795326 & -2.556171 & -0.756589 \\
\hline
\end{tabular}

Title Card Required, conformation \#1

Full file name: amine 38b.log

Method: $\quad B 3 L Y P / 6-31 G(d)$ opt/freq 
Point group: $\quad \mathrm{C} 1$

NImag: $\quad 0$

E(electronic): $\quad-646.184498812$

Zero-point correction=

Thermal correction to Energy=

Thermal correction to Enthalpy=

0.463046 (Hartree/Particle)

Thermal correction to Gibbs Free

Sum of electronic and zero-point Energies=

0.483476

0.484420

Sum of electronic and thermal Energies=

0.418021

$-645.721452$

$-645.701023$

Standard orientation:

$\begin{array}{llcl}\text { Center } & \text { Atomic } & \text { Atomic } & \text { Coordinates (Angstroms) } \\ \text { Number } & \text { Number } & \text { Type } & \mathrm{X}\end{array}$

\begin{tabular}{|c|c|c|c|c|c|}
\hline 1 & 7 & 0 & 1.230925 & 0.117246 & -0.270279 \\
\hline 2 & 6 & 0 & -0.188189 & 0.128812 & -0.784277 \\
\hline 3 & 6 & 0 & -1.190148 & -0.287710 & 0.357142 \\
\hline 4 & 6 & 0 & -2.750208 & -0.147532 & 0.297935 \\
\hline 5 & 6 & 0 & -3.288079 & -0.977932 & 1.488220 \\
\hline 6 & 6 & 0 & -0.323628 & -0.798209 & -2.029606 \\
\hline 7 & 6 & 0 & -0.511014 & 1.526627 & -1.361786 \\
\hline 8 & 6 & 0 & -3.218168 & 1.308581 & 0.512524 \\
\hline 9 & 6 & 0 & -3.389857 & -0.706216 & -0.987655 \\
\hline 10 & 6 & 0 & 1.936254 & -1.131499 & 0.213311 \\
\hline 11 & 6 & 0 & 3.415645 & -1.070506 & -0.258976 \\
\hline 12 & 6 & 0 & 1.899986 & -1.292764 & 1.759438 \\
\hline 13 & 6 & 0 & 1.411410 & -2.467058 & -0.358693 \\
\hline 14 & 6 & 0 & 1.794064 & 1.336950 & 0.378231 \\
\hline 15 & 6 & 0 & 2.330600 & 2.402109 & -0.607927 \\
\hline 16 & 6 & 0 & 1.003694 & 2.003584 & 1.531277 \\
\hline 17 & 1 & 0 & -0.989267 & -1.345611 & 0.561692 \\
\hline 18 & 1 & 0 & -0.881458 & 0.244409 & 1.261774 \\
\hline 19 & 1 & 0 & -4.377932 & -0.883638 & 1.570312 \\
\hline 20 & 1 & 0 & -3.052417 & -2.042771 & 1.370265 \\
\hline 21 & 1 & 0 & -2.851771 & -0.642253 & 2.437253 \\
\hline 22 & 1 & 0 & -1.145806 & -0.458105 & -2.664415 \\
\hline 23 & 1 & 0 & 0.593733 & -0.745766 & -2.623217 \\
\hline 24 & 1 & 0 & -0.526770 & -1.840961 & -1.789470 \\
\hline 25 & 1 & 0 & -0.500963 & 2.333438 & -0.630614 \\
\hline 26 & 1 & 0 & 0.194147 & 1.771085 & -2.161019 \\
\hline 27 & 1 & 0 & -1.508479 & 1.505197 & -1.805863 \\
\hline 28 & 1 & 0 & -2.792621 & 1.726884 & 1.433265 \\
\hline 29 & 1 & 0 & -2.942591 & 1.969345 & -0.313154 \\
\hline 30 & 1 & 0 & -4.310702 & 1.345005 & 0.608561 \\
\hline 31 & 1 & 0 & -3.060006 & -1.731954 & -1.189835 \\
\hline 32 & 1 & 0 & -4.482399 & -0.724115 & -0.886351 \\
\hline 33 & 1 & 0 & -3.157446 & -0.098949 & -1.868069 \\
\hline 34 & 1 & 0 & 3.974665 & -0.228135 & 0.158121 \\
\hline 35 & 1 & 0 & 3.945280 & -1.983178 & 0.038799 \\
\hline 36 & 1 & 0 & 3.453335 & -0.989790 & -1.350235 \\
\hline 37 & 1 & 0 & 2.347348 & -0.444203 & 2.284744 \\
\hline 38 & 1 & 0 & 0.872216 & -1.408323 & 2.117909 \\
\hline 39 & 1 & 0 & 2.461336 & -2.185895 & 2.05925 \\
\hline 40 & 1 & 0 & 2.026630 & -3.268937 & 0.06325 \\
\hline
\end{tabular}

\section{S-97}




\begin{tabular}{rrrrrr}
41 & 1 & 0 & 0.378231 & -2.684165 & -0.080978 \\
42 & 1 & 0 & 1.507988 & -2.523679 & -1.444193 \\
43 & 1 & 0 & 2.695902 & 0.979769 & 0.872136 \\
44 & 1 & 0 & 1.558697 & 3.064477 & -1.005905 \\
45 & 1 & 0 & 3.063938 & 3.036427 & -0.092958 \\
46 & 1 & 0 & 2.832141 & 1.916236 & -1.450478 \\
47 & 1 & 0 & 0.765194 & 1.286647 & 2.322407 \\
48 & 1 & 0 & 1.628381 & 2.788868 & 1.975978 \\
49 & 1 & 0 & 0.070823 & 2.478491 & 1.218044 \\
\hline
\end{tabular}
1
7
0.000000178
$-0.000002394$
$-0.000000069$

Full file name: amine_38bh2o.log

Method: $\quad$ B3LYP/6-31G(d) opt/freq

Point group: $\quad \mathrm{C} 1$

NImag: $\quad 0$

E (electronic): $\quad-722.605586045$

Zero-point correction=

Thermal correction to Energy=

Thermal correction to Enthalpy=

0.489108 (Hartree/Particle)

Thermal correction to Gibbs Free Energy=

Sum of electronic and zero-point Energies=

0.512165

0.513110

Sum of electronic and thermal Energies=

0.441208

$-722.116478$

$-722.093421$

Standard orientation:

$\begin{array}{llll}\text { Center } & \text { Atomic } & \text { Atomic } & \text { Coordinates (Angstroms) } \\ \text { Number } & \text { Number } & \text { Type } & \text { X }\end{array}$

$\begin{array}{rrrrrr}1 & 7 & 0 & -1.102046 & 0.080800 & -0.038297 \\ 2 & 6 & 0 & 0.270181 & 0.225925 & 0.637776 \\ 3 & 6 & 0 & 1.412601 & -0.376679 & -0.257276 \\ 4 & 6 & 0 & 2.948109 & -0.137101 & -0.042184 \\ 5 & 6 & 0 & 3.655686 & -1.158748 & -0.965307 \\ 6 & 6 & 0 & 0.271500 & -0.450874 & 2.041495 \\ 7 & 6 & 0 & 0.480499 & 1.722838 & 0.961082 \\ 8 & 6 & 0 & 3.394006 & 1.269913 & -0.495388 \\ 9 & 6 & 0 & 3.439149 & -0.391376 & 1.395827 \\ 10 & 6 & 0 & -1.671351 & -1.291339 & -0.419785 \\ 11 & 6 & 0 & -3.219832 & -1.227364 & -0.303805 \\ 12 & 6 & 0 & -1.304244 & -1.754004 & -1.854556 \\ 13 & 6 & 0 & -1.271015 & -2.442482 & 0.526876 \\ 14 & 6 & 0 & -1.487895 & 1.105946 & -1.083211 \\ 15 & 6 & 0 & -2.098874 & 2.413878 & -0.524215 \\ 16 & 6 & 0 & -0.493684 & 1.454601 & -2.217155 \\ 17 & 1 & 0 & 1.273318 & -1.463529 & -0.233831 \\ 18 & 1 & 0 & 1.219171 & -0.081201 & -1.289969 \\ 19 & 1 & 0 & 4.743900 & -1.025503 & -0.932847 \\ 20 & 1 & 0 & 3.435921 & -2.189822 & -0.662159 \\ 21 & 1 & 0 & 3.336564 & -1.041636 & -2.008567 \\ 22 & 1 & 0 & 1.025539 & 0.026793 & 2.670952 \\ 23 & 1 & 0 & -0.688689 & -0.324458 & 2.545135\end{array}$




$\begin{array}{rrrrrr}24 & 1 & 0 & 0.517965 & -1.511736 & 2.015806 \\ 25 & 1 & 0 & 0.588314 & 2.360931 & 0.084997 \\ 26 & 1 & 0 & -0.351869 & 2.090374 & 1.567758 \\ 27 & 1 & 0 & 1.389971 & 1.836927 & 1.554804 \\ 28 & 1 & 0 & 3.077274 & 1.470675 & -1.526489 \\ 29 & 1 & 0 & 2.996043 & 2.065534 & 0.138897 \\ 30 & 1 & 0 & 4.488225 & 1.346269 & -0.466793 \\ 31 & 1 & 0 & 3.116730 & -1.371787 & 1.765185 \\ 32 & 1 & 0 & 4.535803 & -0.370240 & 1.426630 \\ 33 & 1 & 0 & 3.081783 & 0.366433 & 2.099519 \\ 34 & 1 & 0 & -3.679813 & -0.461991 & -0.935312 \\ 35 & 1 & 0 & -3.650189 & -2.188524 & -0.606452 \\ 36 & 1 & 0 & -3.512683 & -1.038611 & 0.732946 \\ 37 & 1 & 0 & -1.689956 & -1.083782 & -2.627669 \\ 38 & 1 & 0 & -0.222601 & -1.842579 & -1.986169 \\ 39 & 1 & 0 & -1.742585 & -2.741110 & -2.042611 \\ 40 & 1 & 0 & -1.824941 & -3.334276 & 0.214408 \\ 41 & 1 & 0 & -0.210792 & -2.696388 & 0.474643 \\ 42 & 1 & 0 & -1.540335 & -2.237609 & 1.564084 \\ 43 & 1 & 0 & -2.319436 & 0.634931 & -1.603448 \\ 44 & 1 & 0 & -1.350412 & 3.164455 & -0.260665 \\ 45 & 1 & 0 & -2.741762 & 2.858669 & -1.294197 \\ 46 & 1 & 0 & -2.706387 & 2.229064 & 0.363927 \\ 47 & 1 & 0 & -0.157579 & 0.572961 & -2.768093 \\ 48 & 1 & 0 & -1.011255 & 2.107379 & -2.931531 \\ 49 & 1 & 0 & 0.389856 & 1.997385 & -1.873568 \\ 50 & 1 & 0 & -2.282256 & 0.551218 & 1.545831 \\ 51 & 8 & 0 & -2.760477 & 0.763354 & 2.380357 \\ 52 & 1 & 0 & -3.683712 & 0.873562 & 2.109026 \\ ---------------1\end{array}$

Tri-t-butylamine

Full file name: tritbutylamine.log

Method: B3LYP/6-31G(d) opt/freq

Point group: $\quad$ C3

NImag: $\quad 0$

E (electronic): $\quad-528.224762102$

Thermal correction to Energy=

Thermal correction to Enthalpy=

378490

(Hartree/Particle)

Thermal correction to Gibbs Free Energy=

Sum of electronic and zero-point Energies=

0.394704

0.395648

Sum of electronic and thermal Energies=

0.339826

$-527.846272$

$-527.830058$

Standard orientation:

\begin{tabular}{|c|c|c|c|c|c|}
\hline \multirow{2}{*}{$\begin{array}{l}\text { Center } \\
\text { Number }\end{array}$} & \multirow{2}{*}{$\begin{array}{l}\text { Atomic } \\
\text { Number }\end{array}$} & \multirow{2}{*}{$\begin{array}{c}\text { Atomic } \\
\text { Type }\end{array}$} & \multicolumn{3}{|c|}{ Coordinates (Angstroms) } \\
\hline & & & $\mathrm{x}$ & Y & z \\
\hline 1 & 7 & 0 & 0.000000 & 0.000000 & 0.253444 \\
\hline 2 & 6 & 0 & -0.533105 & 1.412896 & 0.025081 \\
\hline 3 & 6 & 0 & 1.490156 & -0.244765 & 0.025081 \\
\hline
\end{tabular}




\begin{tabular}{|c|c|c|c|c|c|}
\hline 4 & 6 & 0 & -0.957051 & -1.168130 & 0.025081 \\
\hline 5 & 6 & 0 & -1.099540 & 1.657520 & -1.402807 \\
\hline 6 & 6 & 0 & -1.607191 & 1.783285 & 1.101955 \\
\hline 7 & 6 & 0 & 0.510307 & 2.547797 & 0.229918 \\
\hline 8 & 6 & 0 & 1.985225 & 0.123469 & -1.402807 \\
\hline 9 & 6 & 0 & 2.347966 & 0.500226 & 1.101955 \\
\hline 10 & 6 & 0 & 1.951303 & -1.715837 & 0.229918 \\
\hline 11 & 6 & 0 & -0.885685 & -1.780990 & -1.402807 \\
\hline 12 & 6 & 0 & -2.461610 & -0.831960 & 0.229918 \\
\hline 13 & 6 & 0 & -0.740775 & -2.283511 & 1.101955 \\
\hline 14 & 1 & 0 & -1.536165 & 2.662473 & -1.461011 \\
\hline 15 & 1 & 0 & -0.308863 & 1.598123 & -2.156857 \\
\hline 16 & 1 & 0 & -1.878942 & 0.952908 & -1.691868 \\
\hline 17 & 1 & 0 & -1.344858 & 1.319961 & 2.057473 \\
\hline 18 & 1 & 0 & -1.620695 & 2.868524 & 1.249768 \\
\hline 19 & 1 & 0 & -2.625449 & 1.500041 & 0.844947 \\
\hline 20 & 1 & 0 & -0.000000 & 3.488807 & 0.001476 \\
\hline 21 & 1 & 0 & 0.849327 & 2.610828 & 1.265829 \\
\hline 22 & 1 & 0 & 1.377191 & 2.501112 & -0.426012 \\
\hline 23 & 1 & 0 & 1.764713 & 1.150757 & -1.691868 \\
\hline 24 & 1 & 0 & 3.073852 & -0.000879 & -1.461011 \\
\hline 25 & 1 & 0 & 1.538446 & -0.531579 & -2.156857 \\
\hline 26 & 1 & 0 & 2.611798 & 1.523685 & 0.844947 \\
\hline 27 & 1 & 0 & 1.815549 & 0.504701 & 2.057473 \\
\hline 28 & 1 & 0 & 3.294562 & -0.030699 & 1.249768 \\
\hline 29 & 1 & 0 & 3.021396 & -1.744404 & 0.001476 \\
\hline 30 & 1 & 0 & 1.836380 & -2.040953 & 1.265829 \\
\hline 31 & 1 & 0 & 1.477431 & -2.443238 & -0.426012 \\
\hline 32 & 1 & 0 & 0.114229 & -2.103665 & -1.691868 \\
\hline 33 & 1 & 0 & -1.537687 & -2.661594 & -1.461011 \\
\hline 34 & 1 & 0 & -1.229584 & -1.066544 & -2.156857 \\
\hline 35 & 1 & 0 & -2.685707 & -0.569875 & 1.265829 \\
\hline 36 & 1 & 0 & -2.854622 & -0.057874 & -0.426012 \\
\hline 37 & 1 & 0 & -3.021396 & -1.744404 & 0.001476 \\
\hline 38 & 1 & 0 & 0.013651 & -3.023726 & 0.844947 \\
\hline 39 & 1 & 0 & -0.470690 & -1.824662 & 2.057473 \\
\hline 40 & 1 & 0 & -1.673867 & -2.837825 & 1.249768 \\
\hline
\end{tabular}

Tri-t-Butylamine, H-bonded to one water molecule

Full file name: tritbutylamineh2o.log

Method: $\quad$ B3LYP/6-31G(d) opt/freq

Point group: $\quad \mathrm{C} 1$

NImag: $\quad 0$

E (electronic): $\quad-604.646900511$

Zero-point correction=

Thermal correction to Energy=

Thermal correction to Enthalpy=

Thermal correction to Gibbs Free Energy=

Sum of electronic and zero-point Energies=

Sum of electronic and thermal Energies=

0.404132 (Hartree/Particle)
0.423174
0.424118
0.360929
$\quad-604.242769$
$\quad-604.223727$

Standard orientation: 


\begin{tabular}{|c|c|c|c|c|c|}
\hline \multirow{2}{*}{$\begin{array}{l}\text { Center } \\
\text { Number }\end{array}$} & \multirow{2}{*}{$\begin{array}{l}\text { Atomic } \\
\text { Number }\end{array}$} & \multirow{2}{*}{$\begin{array}{l}\text { Atomic } \\
\text { Type }\end{array}$} & \multicolumn{3}{|c|}{ Coordinates (Angstroms) } \\
\hline & & & $\mathrm{x}$ & $\mathrm{Y}$ & Z \\
\hline 1 & 7 & 0 & -0.001911 & -0.004623 & 0.064401 \\
\hline 2 & 6 & 0 & 1.338705 & -0.714162 & -0.264802 \\
\hline 3 & 6 & 0 & -1.300210 & -0.804927 & -0.228832 \\
\hline 4 & 6 & 0 & -0.057396 & 1.520858 & -0.227631 \\
\hline 5 & 6 & 0 & 1.834880 & -0.529719 & -1.724113 \\
\hline 6 & 6 & 0 & 2.475084 & -0.266458 & 0.718237 \\
\hline 7 & 6 & 0 & 1.307163 & -2.249476 & -0.032960 \\
\hline 8 & 6 & 0 & -1.403671 & -1.369862 & -1.670904 \\
\hline 9 & 6 & 0 & -1.498469 & -1.961129 & 0.802183 \\
\hline 10 & 6 & 0 & -2.605492 & 0.009894 & -0.013339 \\
\hline 11 & 6 & 0 & -0.492142 & 1.890751 & -1.671726 \\
\hline 12 & 6 & 0 & 1.298633 & 2.245910 & -0.013898 \\
\hline 13 & 6 & 0 & -0.982900 & 2.262283 & 0.793776 \\
\hline 14 & 1 & 0 & 2.787066 & -1.060017 & -1.847194 \\
\hline 15 & 1 & 0 & 1.132863 & -0.942112 & -2.452787 \\
\hline 16 & 1 & 0 & 2.017625 & 0.511375 & -1.989881 \\
\hline 17 & 1 & 0 & 2.072581 & -0.021540 & 1.703235 \\
\hline 18 & 1 & 0 & 3.184093 & -1.090988 & 0.845793 \\
\hline 19 & 1 & 0 & 3.059393 & 0.581026 & 0.366102 \\
\hline 20 & 1 & 0 & 2.301904 & -2.630013 & -0.283595 \\
\hline 21 & 1 & 0 & 1.124635 & -2.499433 & 1.015406 \\
\hline 22 & 1 & 0 & 0.602828 & -2.795533 & -0.656635 \\
\hline 23 & 1 & 0 & -0.613568 & -2.076443 & -1.924678 \\
\hline 24 & 1 & 0 & -2.354283 & -1.905813 & -1.778460 \\
\hline 25 & 1 & 0 & -1.390114 & -0.573792 & -2.419622 \\
\hline 26 & 1 & 0 & -0.871203 & -2.833732 & 0.650327 \\
\hline 27 & 1 & 0 & -1.346750 & -1.587092 & 1.818076 \\
\hline 28 & 1 & 0 & -2.532894 & -2.314350 & 0.735507 \\
\hline 29 & 1 & 0 & -3.440423 & -0.667261 & -0.217439 \\
\hline 30 & 1 & 0 & -2.708776 & 0.347096 & 1.019454 \\
\hline 31 & 1 & 0 & -2.730280 & 0.858396 & -0.682118 \\
\hline 32 & 1 & 0 & -1.481897 & 1.522238 & -1.940065 \\
\hline 33 & 1 & 0 & -0.524697 & 2.983172 & -1.763350 \\
\hline 34 & 1 & 0 & 0.213367 & 1.521241 & -2.420009 \\
\hline 35 & 1 & 0 & 1.645337 & 2.168619 & 1.018297 \\
\hline 36 & 1 & 0 & 2.093371 & 1.930679 & -0.686767 \\
\hline 37 & 1 & 0 & 1.127010 & 3.306736 & -0.219255 \\
\hline 38 & 1 & 0 & -2.030632 & 2.297328 & 0.505312 \\
\hline 39 & 1 & 0 & -0.908123 & 1.825128 & 1.791467 \\
\hline 40 & 1 & 0 & -0.656508 & 3.304348 & 0.871674 \\
\hline 41 & 8 & 0 & 0.042063 & 0.060035 & 3.037250 \\
\hline 42 & 1 & 0 & 0.381156 & -0.794333 & 3.343380 \\
\hline 43 & 1 & 0 & 0.038683 & -0.016637 & 2.053070 \\
\hline
\end{tabular}

Dimethylamine protonated 
Full file name: h2nme2.log

Method: $\quad$ B3LYP/6-31G(d) opt/freq

Point group: $\quad \mathrm{C} 2 \mathrm{~V}$

NImag: $\quad 0$

E (electronic): $\quad-135.534022483$

Zero-point correction=

Thermal correction to Energy=

0.108610 (Hartree/Particle)

Thermal correction to Enthalpy=

0.113101

Thermal correction to Gibbs Free Energy=

Sum of electronic and zero-point Energies=

0.114045

0.083530

$-135.425412$

Sum of electronic and thermal Energies=

$-135.420921$

Standard orientation:

\begin{tabular}{|c|c|c|c|c|c|}
\hline \multirow{2}{*}{$\begin{array}{l}\text { Center } \\
\text { Number }\end{array}$} & \multirow{2}{*}{$\begin{array}{l}\text { Atomic } \\
\text { Number }\end{array}$} & \multirow{2}{*}{$\begin{array}{l}\text { Atomic } \\
\text { Type }\end{array}$} & \multicolumn{3}{|c|}{ Coordinates (Angstroms) } \\
\hline & & & $\mathrm{x}$ & $\mathrm{Y}$ & Z \\
\hline 1 & 7 & 0 & 0.000000 & -0.000000 & 0.541595 \\
\hline 2 & 6 & 0 & -0.000000 & 1.269089 & -0.276800 \\
\hline 3 & 6 & 0 & -0.000000 & -1.269089 & -0.276800 \\
\hline 4 & 1 & 0 & -0.818265 & 0.000000 & 1.162294 \\
\hline 5 & 1 & 0 & 0.818265 & -0.000000 & 1.162294 \\
\hline 6 & 1 & 0 & 0.000000 & 2.123458 & 0.401381 \\
\hline 7 & 1 & 0 & 0.895370 & 1.275931 & -0.899229 \\
\hline 8 & 1 & 0 & -0.895370 & 1.275931 & -0.899229 \\
\hline 9 & 1 & 0 & -0.000000 & -2.123458 & 0.401381 \\
\hline 10 & 1 & 0 & 0.895370 & -1.275931 & -0.899229 \\
\hline 11 & 1 & 0 & -0.895370 & -1.275931 & -0.899229 \\
\hline
\end{tabular}

Ethyl dimethyl amine protonated

Full file name: etnme2h.log

Method: $\quad$ B3LYP/6-31G(d) opt/freq

Point group: $\quad$ CS

NImag: $\quad 0$

E (electronic): $\quad-214.168601342$

Zero-point correction=

Thermal correction to Energy=

Thermal correction to Enthalpy=

Thermal correction to Gibbs Free Energy=

Sum of electronic and zero-point Energies=

Sum of electronic and thermal Energies=

0.165557 (Hartree/Particle)

0.172403

0.173348

0.135735

$-214.003044$

$-213.996198$

Standard orientation:

$\begin{array}{llll}\text { Center } & \text { Atomic } & \text { Atomic } & \text { Coordinates (Angstroms) } \\ \text { Number } & \text { Number } & \text { Type } & \mathrm{X}\end{array}$

1

7

0

$-0.382550$

0.618310

0.000000 


\begin{tabular}{|c|c|c|c|c|c|}
\hline 2 & 6 & 0 & -0.799998 & -0.856689 & 0.000000 \\
\hline 3 & 6 & 0 & 0.360244 & -1.840018 & -0.000000 \\
\hline 4 & 6 & 0 & 0.360244 & 1.024436 & 1.246026 \\
\hline 5 & 6 & 0 & 0.360244 & 1.024436 & -1.246026 \\
\hline 6 & 1 & 0 & -1.261607 & 1.146596 & 0.000000 \\
\hline 7 & 1 & 0 & -1.428521 & -0.979615 & 0.886173 \\
\hline 8 & 1 & 0 & -1.428521 & -0.979615 & -0.886173 \\
\hline 9 & 1 & 0 & -0.064655 & -2.848636 & -0.000000 \\
\hline 10 & 1 & 0 & 0.986886 & -1.755770 & 0.892118 \\
\hline 11 & 1 & 0 & 0.986886 & -1.755770 & -0.892118 \\
\hline 12 & 1 & 0 & -0.224096 & 0.727467 & 2.118234 \\
\hline 13 & 1 & 0 & 1.331565 & 0.531173 & 1.255782 \\
\hline 14 & 1 & 0 & 0.494020 & 2.107186 & 1.234204 \\
\hline 15 & 1 & 0 & -0.224096 & 0.727467 & -2.118234 \\
\hline 16 & 1 & 0 & 1.331565 & 0.531173 & -1.255782 \\
\hline 17 & 1 & 0 & 0.494020 & 2.107186 & -1.234204 \\
\hline
\end{tabular}

$\begin{array}{lllll}1 & 7 & -0.000001323 & -0.000000611 & -0.000000110\end{array}$

Full file name: tbunme $2 \mathrm{~h} . \log$

Method: B3LYP/6-31G(d) opt/freq

Point group: $\quad \mathrm{C} 1$

NImag: $\quad 0$

E (electronic): $\quad-292.800598140$

Zero-point correction=

Thermal correction to Energy=

0.221399 (Hartree/Particle)

Thermal correction to Enthalpy=

0.230899

Thermal correction to Gibbs Free Energy=

Sum of electronic and zero-point Energies=

0.231843

Sum of electronic and thermal Energies=

0.187502

$-292.579199$

$-292.569699$

Standard orientation:

$\begin{array}{llcl}\text { Center } & \text { Atomic } & \text { Atomic } & \text { Coordinates (Angstroms) } \\ \text { Number } & \text { Number } & \text { Type } & \text { Y }\end{array}$

$\begin{array}{rrrrrr}1 & 7 & 0 & -0.831373 & 0.001217 & -0.407369 \\ 2 & 6 & 0 & 0.692270 & -0.000543 & 0.024425 \\ 3 & 6 & 0 & 0.797724 & -0.091126 & 1.548198 \\ 4 & 6 & 0 & 1.350919 & -1.208805 & -0.657789 \\ 5 & 6 & 0 & 1.317548 & 1.298190 & -0.505068 \\ 6 & 6 & 0 & -1.605245 & -1.228873 & -0.008852 \\ 7 & 6 & 0 & -1.607341 & 1.229553 & -0.007352 \\ 8 & 1 & 0 & -0.795953 & 0.002263 & -1.432562 \\ 9 & 1 & 0 & 1.858616 & -0.065723 & 1.814584 \\ 10 & 1 & 0 & 0.322202 & 0.752931 & 2.056529 \\ 11 & 1 & 0 & 0.391394 & -1.025761 & 1.945062 \\ 12 & 1 & 0 & 1.188031 & -1.210180 & -1.742712 \\ 13 & 1 & 0 & 2.430706 & -1.148439 & -0.497041 \\ 14 & 1 & 0 & 1.022686 & -2.166715 & -0.246735 \\ 15 & 1 & 0 & 1.137303 & 1.437561 & -1.578150 \\ 16 & 1 & 0 & 2.400612 & 1.238555 & -0.368083\end{array}$




$\begin{array}{rrrrrr}17 & 1 & 0 & 0.976634 & 2.186208 & 0.032995 \\ 18 & 1 & 0 & -1.714873 & -1.242155 & 1.074941 \\ 19 & 1 & 0 & -2.589928 & -1.178575 & -0.476097 \\ 20 & 1 & 0 & -1.085493 & -2.121633 & -0.349229 \\ 21 & 1 & 0 & -1.638342 & 1.291395 & 1.079978 \\ 22 & 1 & 0 & -2.621688 & 1.134456 & -0.397763 \\ 23 & 1 & 0 & -1.137552 & 2.116920 & -0.425502 \\ -\end{array}$

Di-t-butylmethylamine protonated

Full file name: ditbunmeh.log

Method: B3LYP/6-31G(d) opt/freq

Point group: $\quad \mathrm{C} 1$

NImag: $\quad 0$

E(electronic): $\quad-410.732584885$

Zero-point correction=

0.307021 (Hartree/Particle)

Thermal correction to Energy=

0.319878

Thermal correction to Enthalpy=

0.320822

Thermal correction to Gibbs Free Energy=

Sum of electronic and zero-point Energies=

0.270660

Sum of electronic and thermal Energies=

$-410.425564$

$-410.412707$

Standard orientation:

\begin{tabular}{|c|c|c|c|c|c|}
\hline \multirow{2}{*}{$\begin{array}{l}\text { Center } \\
\text { Number }\end{array}$} & \multirow{2}{*}{$\begin{array}{l}\text { Atomic } \\
\text { Number }\end{array}$} & \multirow{2}{*}{$\begin{array}{l}\text { Atomic } \\
\text { Type }\end{array}$} & \multicolumn{3}{|c|}{ Coordinates (Angstroms) } \\
\hline & & & $\mathrm{x}$ & $\mathrm{Y}$ & Z \\
\hline 1 & 7 & 0 & 0.007329 & 0.481801 & -0.406137 \\
\hline 2 & 6 & 0 & -0.001304 & 1.964289 & -0.117640 \\
\hline 3 & 6 & 0 & -1.407374 & -0.161751 & -0.006414 \\
\hline 4 & 6 & 0 & 1.407257 & -0.179323 & 0.000455 \\
\hline 5 & 6 & 0 & -1.822597 & 0.286226 & 1.400805 \\
\hline 6 & 6 & 0 & -2.404825 & 0.349860 & -1.065244 \\
\hline 7 & 6 & 0 & -1.386370 & -1.692606 & -0.090618 \\
\hline 8 & 6 & 0 & 1.471537 & -0.444699 & 1.505961 \\
\hline 9 & 6 & 0 & 2.522307 & 0.807867 & -0.402693 \\
\hline 10 & 6 & 0 & 1.615904 & -1.462023 & -0.823673 \\
\hline 11 & 1 & 0 & 0.011385 & 0.397535 & -1.427323 \\
\hline 12 & 1 & 0 & 0.713906 & 2.463894 & -0.765855 \\
\hline 13 & 1 & 0 & -0.989700 & 2.369838 & -0.311904 \\
\hline 14 & 1 & 0 & 0.261506 & 2.130151 & 0.925457 \\
\hline 15 & 1 & 0 & -2.789116 & -0.175867 & 1.622917 \\
\hline 16 & 1 & 0 & -1.122500 & -0.041235 & 2.171561 \\
\hline 17 & 1 & 0 & -1.962112 & 1.366317 & 1.485081 \\
\hline 18 & 1 & 0 & -3.377440 & -0.110162 & -0.869964 \\
\hline 19 & 1 & 0 & -2.108726 & 0.053324 & -2.079078 \\
\hline 20 & 1 & 0 & -2.560220 & 1.431216 & -1.046910 \\
\hline 21 & 1 & 0 & -2.407986 & -2.037957 & 0.093514 \\
\hline 22 & 1 & 0 & -1.100826 & -2.054873 & -1.081605 \\
\hline 23 & 1 & 0 & -0.753687 & -2.159166 & 0.665334 \\
\hline 24 & 1 & 0 & 2.480259 & -0.797912 & 1.741375 \\
\hline 25 & 1 & 0 & 0.774679 & -1.217907 & 1.833931 \\
\hline
\end{tabular}

\section{S-104}




$\begin{array}{rrrrrr}26 & 1 & 0 & 1.304322 & 0.459834 & 2.098570 \\ 27 & 1 & 0 & 3.479315 & 0.300155 & -0.256752 \\ 28 & 1 & 0 & 2.468748 & 1.089826 & -1.460781 \\ 29 & 1 & 0 & 2.545971 & 1.711553 & 0.209414 \\ 30 & 1 & 0 & 2.660342 & -1.766469 & -0.712940 \\ 31 & 1 & 0 & 1.449536 & -1.290659 & -1.894568 \\ 32 & 1 & 0 & 1.003827 & -2.301079 & -0.502139 \\ -\end{array}$

Amine 38b from Banert paper (N-(tert-butyl)-N-isopropyl-2,4,4-trimethy

Full file name: amine 38bh.log

Method: $\quad$ B3LYP/ $6-31 \mathrm{G}(\mathrm{d})$ opt/freq

Point group: $\quad$ C1

NImag: $\quad 0$

E (electronic) : $\quad-646.590322097$

Zero-point correction=

Thermal correction to Energy=

0.478128 (Hartree/Particle)

Thermal correction to Enthalpy=

0.498690

Thermal correction to Gibbs Free Energy=

Sum of electronic and zero-point Energies=

0.499634

Sum of electronic and thermal Energies=

$-646.112194$

$-646.091632$

Standard orientation:

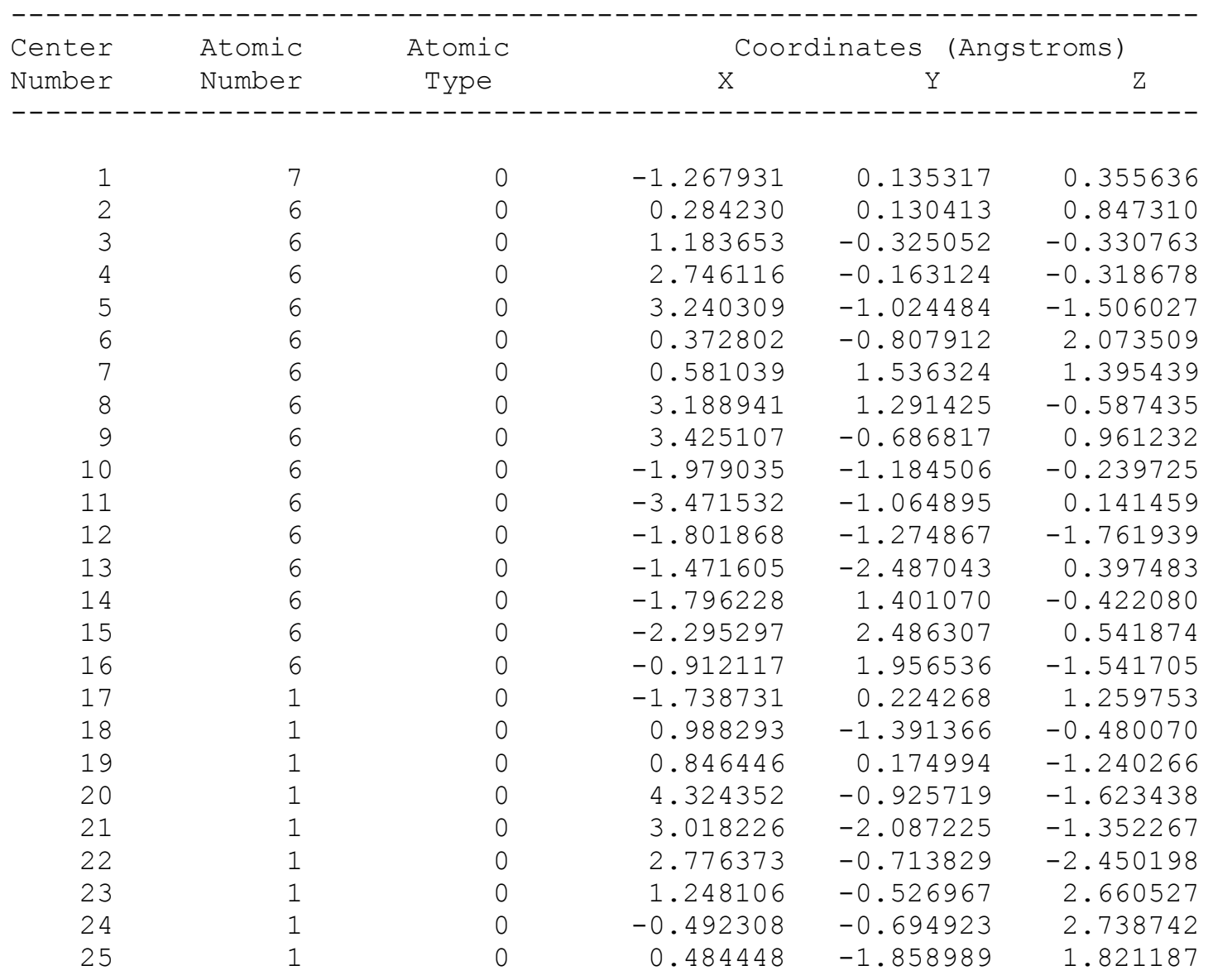




$\begin{array}{rrrrrr}26 & 1 & 0 & 0.574729 & 2.328802 & 0.651645 \\ 27 & 1 & 0 & -0.109113 & 1.797900 & 2.204147 \\ 28 & 1 & 0 & 1.579566 & 1.515426 & 1.834116 \\ 29 & 1 & 0 & 2.727300 & 1.686941 & -1.500690 \\ 30 & 1 & 0 & 2.957924 & 1.974253 & 0.234446 \\ 31 & 1 & 0 & 4.274085 & 1.326003 & -0.732546 \\ 32 & 1 & 0 & 3.112751 & -1.709749 & 1.201209 \\ 33 & 1 & 0 & 4.511225 & -0.703415 & 0.820445 \\ 34 & 1 & 0 & 3.232383 & -0.054415 & 1.834753 \\ 35 & 1 & 0 & -3.990044 & -0.215620 & -0.309186 \\ 36 & 1 & 0 & -3.986040 & -1.965595 & -0.204758 \\ 37 & 1 & 0 & -3.605694 & -1.018356 & 1.229174 \\ 38 & 1 & 0 & -2.264041 & -0.449036 & -2.306478 \\ 39 & 1 & 0 & -0.752527 & -1.350024 & -2.053511 \\ 40 & 1 & 0 & -2.299464 & -2.189434 & -2.098357 \\ 41 & 1 & 0 & -2.086532 & -3.295004 & -0.010131 \\ 42 & 1 & 0 & -0.437640 & -2.721005 & 0.144393 \\ 43 & 1 & 0 & -1.598219 & -2.507597 & 1.481606 \\ 44 & 1 & 0 & -2.684182 & 1.014785 & -0.912968 \\ 45 & 1 & 0 & -1.498883 & 3.083295 & 0.986220 \\ 46 & 1 & 0 & -2.937203 & 3.167719 & -0.025703 \\ 47 & 1 & 0 & -2.912168 & 2.068847 & 1.347069 \\ 48 & 1 & 0 & -0.670427 & 1.209978 & -2.299828 \\ 49 & 1 & 0 & -1.497762 & 2.737838 & -2.038375 \\ 50 & 1 & 0 & 0.013195 & 2.419764 & -1.199835 \\ --------------------------------------------------\end{array}$

Tri-t-butylamine protonated

Full file name: tritbutylamineh.log

Method: B3LYP/6-31G(d) opt/freq

Point group: $\quad$ C3

NImag: $\quad 0$

E (electronic): $\quad-528.632428224$

Zero-point correction=

Thermal correction to Energy=

Thermal correction to Enthalpy=

Thermal correction to Gibbs Free Energy=

Sum of electronic and zero-point Energies=

Sum of electronic and thermal Energies=

0.392703 (Hartree/Particle)
0.409425
0.410370
0.353387
$\quad-528.239725$
$\quad-528.223003$

Standard orientation:

$\begin{array}{llcl}\text { Center } & \text { Atomic } & \text { Atomic } & \text { Coordinates (Angstroms) } \\ \text { Number } & \text { Number } & \text { Type } & \text { X }\end{array}$

$\begin{array}{rrrrrr}1 & 7 & 0 & 0.000000 & 0.000000 & 0.327154 \\ 2 & 1 & 0 & 0.000000 & 0.000000 & 1.349843 \\ 3 & 6 & 0 & -0.562316 & 1.505164 & 0.015002 \\ 4 & 6 & 0 & 1.584668 & -0.265602 & 0.015002 \\ 5 & 6 & 0 & -1.022352 & -1.239562 & 0.015002 \\ 6 & 6 & 0 & -1.078825 & 1.628548 & -1.425242 \\ 7 & 6 & 0 & -1.655818 & 1.838543 & 1.063223\end{array}$

\section{S-106}




\begin{tabular}{|c|c|c|c|c|c|}
\hline 8 & 6 & 0 & 0.501499 & 2.604143 & 0.231061 \\
\hline 9 & 6 & 0 & 1.949777 & 0.120016 & -1.425242 \\
\hline 10 & 6 & 0 & 2.420134 & 0.514709 & 1.063223 \\
\hline 11 & 6 & 0 & 2.004505 & -1.736383 & 0.231061 \\
\hline 12 & 6 & 0 & -0.870951 & -1.748564 & -1.425242 \\
\hline 13 & 6 & 0 & -2.506004 & -0.867760 & 0.231061 \\
\hline 14 & 6 & 0 & -0.764316 & -2.353252 & 1.063223 \\
\hline 15 & 1 & 0 & -1.445656 & 2.651707 & -1.554427 \\
\hline 16 & 1 & 0 & -0.291105 & 1.472703 & -2.164390 \\
\hline 17 & 1 & 0 & -1.908558 & 0.965719 & -1.661170 \\
\hline 18 & 1 & 0 & -1.375532 & 1.501858 & 2.068956 \\
\hline 19 & 1 & 0 & -1.746766 & 2.926264 & 1.118610 \\
\hline 20 & 1 & 0 & -2.643760 & 1.455815 & 0.830918 \\
\hline 21 & 1 & 0 & 0.000000 & 3.550011 & 0.008477 \\
\hline 22 & 1 & 0 & 0.838466 & 2.663742 & 1.267915 \\
\hline 23 & 1 & 0 & 1.360499 & 2.551320 & -0.432450 \\
\hline 24 & 1 & 0 & 1.790617 & 1.170000 & -1.661170 \\
\hline 25 & 1 & 0 & 3.019274 & -0.073879 & -1.554427 \\
\hline 26 & 1 & 0 & 1.420951 & -0.484247 & -2.164390 \\
\hline 27 & 1 & 0 & 2.582653 & 1.561656 & 0.830918 \\
\hline 28 & 1 & 0 & 1.988413 & 0.440316 & 2.068956 \\
\hline 29 & 1 & 0 & 3.407602 & 0.049612 & 1.118610 \\
\hline 30 & 1 & 0 & 3.074400 & -1.775005 & 0.008477 \\
\hline 31 & 1 & 0 & 1.887635 & -2.058004 & 1.267915 \\
\hline 32 & 1 & 0 & 1.529259 & -2.453887 & -0.432450 \\
\hline 33 & 1 & 0 & 0.117942 & -2.135720 & -1.661170 \\
\hline 34 & 1 & 0 & -1.573618 & -2.577828 & -1.554427 \\
\hline 35 & 1 & 0 & -1.129845 & -0.988456 & -2.164390 \\
\hline 36 & 1 & 0 & -2.726101 & -0.605738 & 1.267915 \\
\hline 37 & 1 & 0 & -2.889758 & -0.097433 & -0.432450 \\
\hline 38 & 1 & 0 & -3.074400 & -1.775005 & 0.008477 \\
\hline 39 & 1 & 0 & 0.061108 & -3.017471 & 0.830918 \\
\hline 40 & 1 & 0 & -0.612881 & -1.942175 & 2.068956 \\
\hline 41 & 1 & 0 & -1.660835 & -2.975876 & 1.118610 \\
\hline
\end{tabular}

Ethene

Full file name: ethene.log

Method: $\quad$ B3LYP/6-31G(d) opt/freq

Point group: D2H

NImag: 0

E (electronic) : $\quad-78.5874586870$

Zero-point correction=

Thermal correction to Energy=

0.051223 (Hartree/Particle)

Thermal correction to Enthalpy=

0.054265

Thermal correction to Gibbs Free Energy=

0.055209

Sum of electronic and zero-point Energies=

0.030348

$-78.536236$

Sum of electronic and thermal Energies=

$-78.533194$ 
Standard orientation:

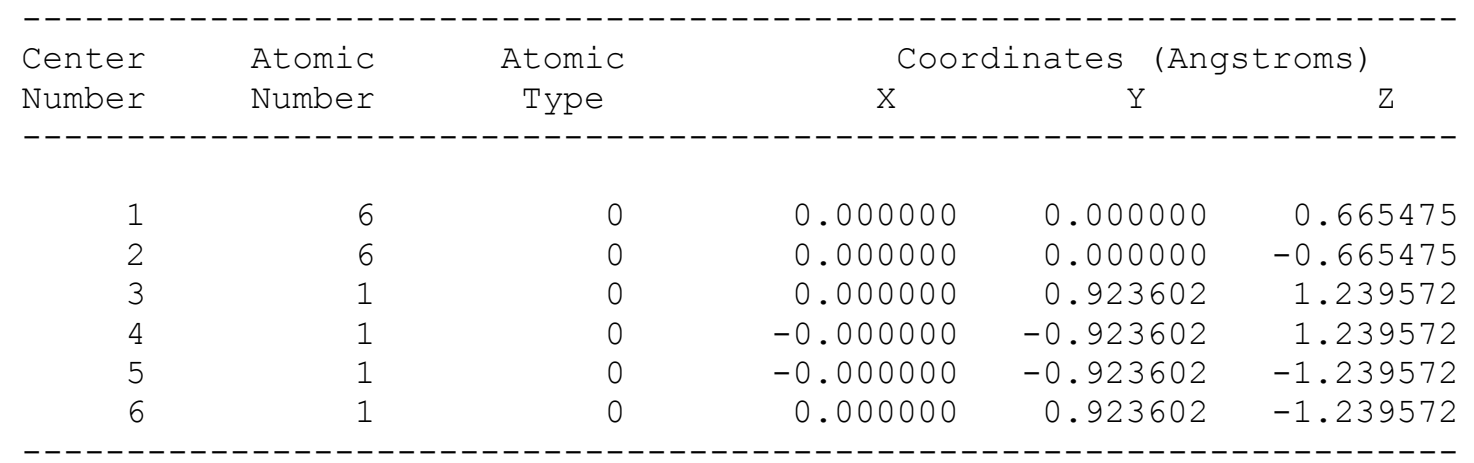

2,4,4-Trimethylpent-1-ene

Full file name: 244 tmpentlene.log

Method: B3LYP/6-31G(d) opt/freq

Point group: CS

NImag: $\quad 0$

E(electronic): $\quad-314.474176988$

Zero-point correction=

0.222302 (Hartree/Particle)

Thermal correction to Energy=

0.232690

Thermal correction to Enthalpy=

0.233634

Thermal correction to Gibbs Free Energy=

Sum of electronic and zero-point Energies=

0.187732

Sum of electronic and thermal Energies=

$-314.251875$

$-314.241487$

Standard orientation:

\begin{tabular}{|c|c|c|c|c|c|}
\hline \multirow{2}{*}{$\begin{array}{l}\text { Center } \\
\text { Number }\end{array}$} & \multirow{2}{*}{$\begin{array}{l}\text { Atomic } \\
\text { Number }\end{array}$} & \multirow{2}{*}{$\begin{array}{c}\text { Atomic } \\
\text { Type }\end{array}$} & \multicolumn{3}{|c|}{ Coordinates (Angstroms) } \\
\hline & & & $\mathrm{X}$ & Y & Z \\
\hline 1 & 6 & 0 & -1.023619 & 1.956705 & 0.000000 \\
\hline 2 & 6 & 0 & 0.252696 & 1.556988 & 0.000000 \\
\hline 3 & 6 & 0 & 1.365530 & 2.583651 & 0.000000 \\
\hline 4 & 6 & 0 & 0.775087 & 0.129203 & 0.000000 \\
\hline 5 & 6 & 0 & -0.150904 & -1.116602 & 0.000000 \\
\hline 6 & 6 & 0 & 0.774261 & -2.354264 & 0.000000 \\
\hline 7 & 6 & 0 & -1.023619 & -1.181342 & 1.270041 \\
\hline 8 & 6 & 0 & -1.023619 & -1.181342 & -1.270041 \\
\hline 9 & 1 & 0 & -1.271713 & 3.015514 & 0.000000 \\
\hline 10 & 1 & 0 & -1.865126 & 1.275148 & 0.000000 \\
\hline 11 & 1 & 0 & 0.974657 & 3.605622 & 0.000000 \\
\hline 12 & 1 & 0 & 2.013131 & 2.466448 & 0.880017 \\
\hline 13 & 1 & 0 & 2.013131 & 2.466448 & -0.880017 \\
\hline 14 & 1 & 0 & 1.447106 & 0.039987 & 0.869538 \\
\hline 15 & 1 & 0 & 1.447106 & 0.039987 & -0.869538 \\
\hline 16 & 1 & 0 & 0.185724 & -3.279624 & 0.000000 \\
\hline 17 & 1 & 0 & 1.420577 & -2.369365 & 0.886453 \\
\hline 18 & 1 & 0 & 1.420577 & -2.369365 & -0.886453 \\
\hline 19 & 1 & 0 & -0.398411 & -1.165142 & 2.171578 \\
\hline 20 & 1 & 0 & -1.726480 & -0.347791 & 1.343518 \\
\hline
\end{tabular}

\section{S-108}




\begin{tabular}{|c|c|c|c|c|c|}
\hline 21 & 1 & 0 & -1.605142 & -2.111456 & 1.287020 \\
\hline 22 & 1 & 0 & -0.398411 & -1.165142 & -2.171578 \\
\hline 23 & 1 & 0 & -1.726480 & -0.347791 & -1.343518 \\
\hline 24 & 1 & 0 & -1.605142 & -2.111456 & -1.287020 \\
\hline
\end{tabular}

Isobutene

Full file name: isobutene.log

Method: B3LYP/6-31G(d) opt/freq

Point group: $\quad \mathrm{C} 2 \mathrm{~V}$

NImag: $\quad 0$

E (electronic): $\quad-157.227291532$

Zero-point correction=

Thermal correction to Energy=

0.108526 (Hartree/Particle)

Thermal correction to Enthalpy=

0.113814

Thermal correction to Gibbs Free Energy=

0.114758

Sum of electronic and zero-point Energies=

0.081897

Sum of electronic and thermal Energies=

$-157.118766$

$-157.113478$

Standard orientation:

$\begin{array}{llll}\text { Center } & \text { Atomic } & \text { Atomic } & \text { Coordinates (Angstroms) } \\ \text { Number } & \text { Number } & \text { Type } & \text { X } \\ -\end{array}$

$\begin{array}{rrrrrr}1 & 6 & 0 & 0.000000 & 0.000000 & 0.122959 \\ 2 & 6 & 0 & 0.000000 & 0.000000 & 1.459591 \\ 3 & 6 & 0 & 0.000000 & 1.278039 & -0.678956 \\ 4 & 6 & 0 & -0.000000 & -1.278039 & -0.678956 \\ 5 & 1 & 0 & 0.000000 & 0.924673 & 2.031664 \\ 6 & 1 & 0 & -0.000000 & -0.924673 & 2.031664 \\ 7 & 1 & 0 & 0.000000 & 2.163243 & -0.035593 \\ 8 & 1 & 0 & 0.880000 & 1.332287 & -1.334993 \\ 9 & 1 & 0 & -0.880000 & 1.332287 & -1.334993 \\ 10 & 1 & 0 & -0.000000 & -2.163243 & -0.035593 \\ 11 & 1 & 0 & -0.880000 & -1.332287 & -1.334993 \\ 12 & 1 & 0 & 0.880000 & -1.332287 & -1.334993 \\ - & 1 & 0 & & \end{array}$

Ethyl cation

Full file name: etcat.log

Method: B3LYP/6-31G(d) opt/freq

Point group: $\quad \mathrm{C} 2 \mathrm{~V}$

NImag: $\quad 0$

E(electronic): $\quad-78.8601098544$

Zero-point correction=

0.061011 (Hartree/Particle) 
Thermal correction to Energy=

Thermal correction to Enthalpy=

Thermal correction to Gibbs Free Energy=

Sum of electronic and zero-point Energies=

Sum of electronic and thermal Energies=
0.064222
0.065166
0.039129
-78.799099
-78.795888

Standard orientation:

$\begin{array}{llcl}\text { Center } & \text { Atomic } & \text { Atomic Coordinates (Angstroms) } \\ \text { Number } & \text { Number } & \text { Type } & \text { X }\end{array}$

\begin{tabular}{|c|c|c|c|c|c|}
\hline 1 & 1 & 0 & 0.000000 & 0.000000 & 1.060936 \\
\hline 2 & 6 & 0 & 0.000000 & 0.691413 & -0.063430 \\
\hline 3 & 6 & 0 & -0.000000 & -0.691413 & -0.063430 \\
\hline 4 & 1 & 0 & 0.936585 & 1.249420 & -0.074943 \\
\hline 5 & 1 & 0 & -0.936585 & 1.249420 & -0.074943 \\
\hline 6 & 1 & 0 & -0.936585 & -1.249420 & -0.074943 \\
\hline 7 & 1 & 0 & 0.936585 & -1.249420 & -0.074943 \\
\hline
\end{tabular}

2,4,4-Trimethylpent-1-ene cation (protonated to give tertiary carbocat

Full file name: 244 tmpentlene_cat.log

Method: B3LYP/6-31G(d) opt/freq

Point group: $\quad$ C1

NImag: $\quad 0$

E (electronic): $\quad-314.817878501$

Zero-point correction=

Thermal correction to Energy=

0.232109 (Hartree/Particle)

Thermal correction to Enthalpy=

0.243269

Thermal correction to Gibbs Free Energy=

0.244213

Sum of electronic and zero-point Energies=

0.196496

Sum of electronic and thermal Energies=

$-314.585769$

$-314.574610$

Standard orientation:

$\begin{array}{llll}\text { Center } & \text { Atomic } & \text { Atomic } & \text { Coordinates (Angstroms) } \\ \text { Number } & \text { Number } & \text { Type } & \text { Y }\end{array}$

$\begin{array}{rrrrrr}1 & 6 & 0 & -2.060906 & 1.293131 & 0.042609 \\ 2 & 6 & 0 & -1.445089 & -0.004732 & -0.289419 \\ 3 & 6 & 0 & -2.167659 & -1.236345 & 0.081449 \\ 4 & 6 & 0 & -0.179641 & -0.084096 & -0.980469 \\ 5 & 6 & 0 & 1.127161 & -0.004962 & 0.048614 \\ 6 & 6 & 0 & 2.331017 & -0.166282 & -0.895411 \\ 7 & 6 & 0 & 1.176527 & 1.356401 & 0.750167 \\ 8 & 6 & 0 & 1.080112 & -1.155257 & 1.059988 \\ 9 & 1 & 0 & -2.195747 & 1.364977 & 1.133601 \\ 10 & 1 & 0 & -1.507733 & 2.156724 & -0.325190 \\ 11 & 1 & 0 & -3.087842 & 1.310110 & -0.357400 \\ 12 & 1 & 0 & -2.872681 & -1.100942 & 0.905766\end{array}$

\section{S-110}




\begin{tabular}{|c|c|c|c|c|c|}
\hline 13 & 1 & 0 & -2.763270 & -1.510649 & -0.810522 \\
\hline 14 & 1 & 0 & -1.496189 & -2.081307 & 0.258484 \\
\hline 15 & 1 & 0 & -0.041915 & 0.763609 & -1.657122 \\
\hline 16 & 1 & 0 & -0.065332 & -1.037650 & -1.502202 \\
\hline 17 & 1 & 0 & 3.249448 & -0.118939 & -0.299255 \\
\hline 18 & 1 & 0 & 2.375963 & 0.633111 & -1.641833 \\
\hline 19 & 1 & 0 & 2.318861 & -1.130288 & -1.413794 \\
\hline 20 & 1 & 0 & 1.165334 & 2.187709 & 0.036979 \\
\hline 21 & 1 & 0 & 0.362365 & 1.495623 & 1.468880 \\
\hline 22 & 1 & 0 & 2.113220 & 1.425994 & 1.314036 \\
\hline 23 & 1 & 0 & 0.988686 & -2.131807 & 0.571034 \\
\hline 24 & 1 & 0 & 0.269032 & -1.046721 & 1.788563 \\
\hline 25 & 1 & 0 & 2.018667 & -1.166704 & 1.624806 \\
\hline
\end{tabular}
1
6
0.000007889
$-0.000000000$
0.000002241

Full file name: tbucat.log

Method: B3LYP/6-31G(d) opt/freq

Point group: CS

NImag: 1

E (electronic) : $\quad-157.554059220$

Thermal correction to Energy=

0.117278 (Hartree/Particle)

Thermal correction to Enthalpy=

0.122627

Thermal correction to Gibbs Free Energy=

Sum of electronic and zero-point Energies=

0.123571

Sum of electronic and thermal Energies=

$-157.436781$

$-157.431432$

Standard orientation:

\begin{tabular}{|c|c|c|c|c|c|}
\hline \multirow{2}{*}{$\begin{array}{l}\text { Center } \\
\text { Number }\end{array}$} & \multirow{2}{*}{$\begin{array}{l}\text { Atomic } \\
\text { Number }\end{array}$} & \multirow{2}{*}{$\begin{array}{c}\text { Atomic } \\
\text { Type }\end{array}$} & \multicolumn{3}{|c|}{ Coordinates (Angstroms) } \\
\hline & & & $\mathrm{X}$ & $\mathrm{Y}$ & Z \\
\hline & & & & & -- \\
\hline 1 & 6 & 0 & 0.017822 & -0.005137 & 0.000000 \\
\hline 2 & 6 & 0 & -0.021947 & 1.459365 & -0.000000 \\
\hline 3 & 1 & 0 & 1.048061 & 1.759165 & -0.000000 \\
\hline 4 & 6 & 0 & 0.017822 & -0.730939 & 1.274357 \\
\hline 5 & 6 & 0 & 0.017822 & -0.730939 & -1.274357 \\
\hline 6 & 1 & 0 & -0.454980 & 1.885388 & -0.909779 \\
\hline 7 & 1 & 0 & -0.454980 & 1.885388 & 0.909779 \\
\hline 8 & 1 & 0 & -1.055321 & -0.874928 & 1.520738 \\
\hline 9 & 1 & 0 & 0.463760 & -1.726427 & 1.215520 \\
\hline 10 & 1 & 0 & 0.427953 & -0.140669 & 2.100280 \\
\hline 11 & 1 & 0 & -1.055321 & -0.874928 & -1.520738 \\
\hline 12 & 1 & 0 & 0.463760 & -1.726427 & -1.215520 \\
\hline 13 & 1 & 0 & 0.427953 & -0.140669 & -2.100280 \\
\hline
\end{tabular}




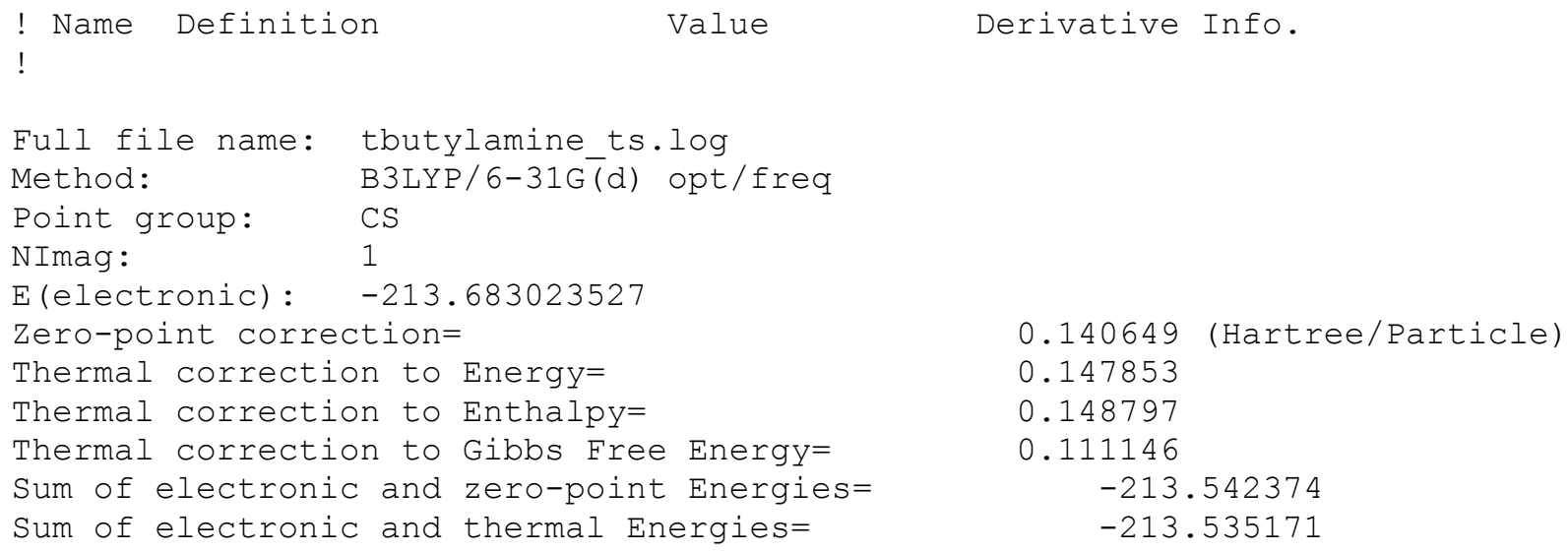

Standard orientation:

\begin{tabular}{|c|c|c|c|c|c|}
\hline \multirow{2}{*}{$\begin{array}{l}\text { Center } \\
\text { Number }\end{array}$} & \multirow{2}{*}{$\begin{array}{l}\text { Atomic } \\
\text { Number }\end{array}$} & \multirow{2}{*}{$\begin{array}{c}\text { Atomic } \\
\text { Type }\end{array}$} & \multicolumn{3}{|c|}{ Coordinates (Angstroms) } \\
\hline & & & $\mathrm{X}$ & $\mathrm{Y}$ & Z \\
\hline & & & & & --- \\
\hline 1 & 7 & 0 & -0.476853 & -1.495867 & 0.000000 \\
\hline 2 & 6 & 0 & 0.106731 & 0.197124 & -0.000000 \\
\hline 3 & 6 & 0 & -0.476853 & 0.789426 & 1.273833 \\
\hline 4 & 6 & 0 & 1.528443 & -0.156072 & 0.000000 \\
\hline 5 & 6 & 0 & -0.476853 & 0.789426 & -1.273833 \\
\hline 6 & 1 & 0 & -0.978020 & -1.824143 & 0.833795 \\
\hline 7 & 1 & 0 & -0.978020 & -1.824143 & -0.833795 \\
\hline 8 & 1 & 0 & -0.162111 & 1.837472 & 1.350283 \\
\hline 9 & 1 & 0 & -1.573337 & 0.766371 & 1.294042 \\
\hline 10 & 1 & 0 & -0.095048 & 0.269800 & 2.159506 \\
\hline 11 & 1 & 0 & 0.717044 & -1.544442 & 0.000000 \\
\hline 12 & 1 & 0 & 2.074577 & 0.098541 & -0.909826 \\
\hline 13 & 1 & 0 & 2.074577 & 0.098541 & 0.909826 \\
\hline 14 & 1 & 0 & -0.162111 & 1.837472 & -1.350283 \\
\hline 15 & 1 & 0 & -0.095048 & 0.269800 & -2.159506 \\
\hline 16 & 1 & 0 & -1.573337 & 0.766371 & -1.294042 \\
\hline
\end{tabular}

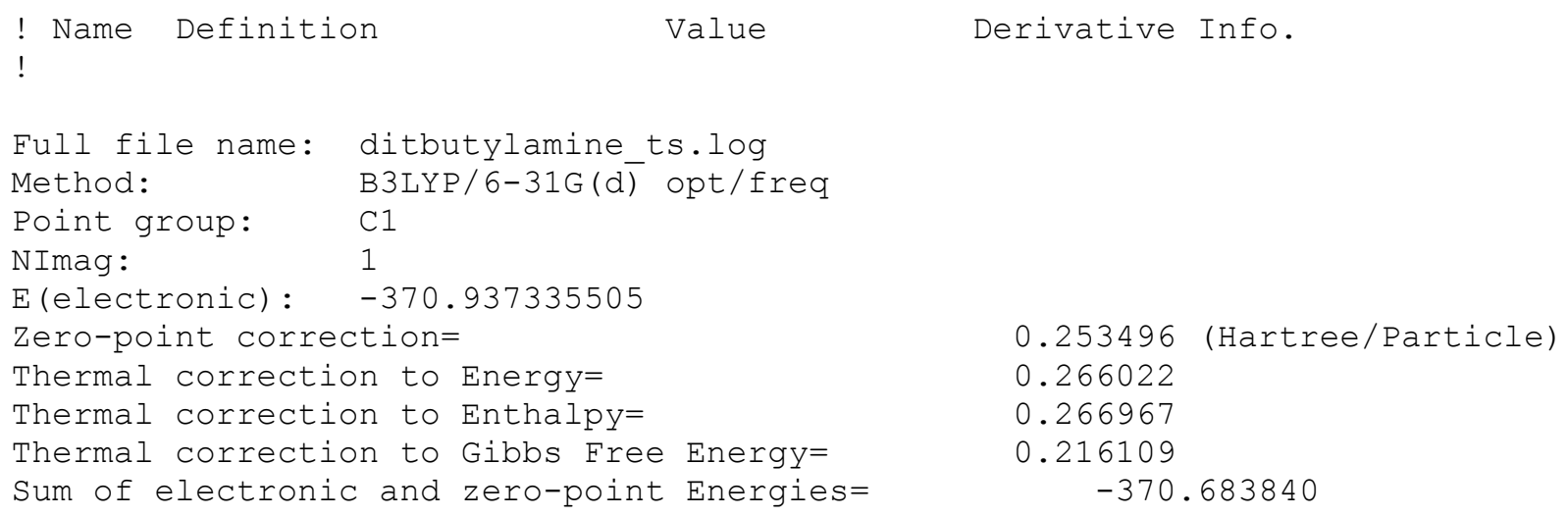


Standard orientation:

\begin{tabular}{|c|c|c|c|c|c|}
\hline \multirow{2}{*}{$\begin{array}{l}\text { Center } \\
\text { Number }\end{array}$} & \multirow{2}{*}{$\begin{array}{l}\text { Atomic } \\
\text { Number }\end{array}$} & \multirow{2}{*}{$\begin{array}{c}\text { Atomic } \\
\text { Type }\end{array}$} & \multicolumn{3}{|c|}{ Coordinates (Angstroms) } \\
\hline & & & $\mathrm{X}$ & Y & Z \\
\hline & & & & & ---- \\
\hline 1 & 7 & 0 & -0.199099 & -0.294005 & 0.493855 \\
\hline 2 & 6 & 0 & 1.901404 & 0.023085 & 0.066138 \\
\hline 3 & 6 & 0 & 1.882914 & 1.221305 & -0.841939 \\
\hline 4 & 6 & 0 & 2.023877 & 0.210261 & 1.478170 \\
\hline 5 & 6 & 0 & 2.296086 & -1.286515 & -0.565170 \\
\hline 6 & 6 & 0 & -1.568755 & -0.029151 & -0.000999 \\
\hline 7 & 6 & 0 & -1.768244 & -0.692158 & -1.378813 \\
\hline 8 & 6 & 0 & -2.597971 & -0.593981 & 1.000518 \\
\hline 9 & 6 & 0 & -1.749588 & 1.492968 & -0.120657 \\
\hline 10 & 1 & 0 & -0.132057 & -1.313609 & 0.620645 \\
\hline 11 & 1 & 0 & 2.920843 & 1.505622 & -1.077104 \\
\hline 12 & 1 & 0 & 1.372256 & 1.017540 & -1.788111 \\
\hline 13 & 1 & 0 & 1.407960 & 2.079278 & -0.359229 \\
\hline 14 & 1 & 0 & 0.678800 & 0.096543 & 1.503674 \\
\hline 15 & 1 & 0 & 2.498100 & -0.586246 & 2.052146 \\
\hline 16 & 1 & 0 & 2.265595 & 1.216190 & 1.823783 \\
\hline 17 & 1 & 0 & 3.371557 & -1.258545 & -0.801991 \\
\hline 18 & 1 & 0 & 2.139187 & -2.129323 & 0.115564 \\
\hline 19 & 1 & 0 & 1.759079 & -1.477392 & -1.499405 \\
\hline 20 & 1 & 0 & -1.029678 & -0.320156 & -2.098204 \\
\hline 21 & 1 & 0 & -1.654511 & -1.781509 & -1.309334 \\
\hline 22 & 1 & 0 & -2.767959 & -0.485696 & -1.781848 \\
\hline 23 & 1 & 0 & -2.451519 & -0.150733 & 1.991630 \\
\hline 24 & 1 & 0 & -3.626113 & -0.383559 & 0.677997 \\
\hline 25 & 1 & 0 & -2.493467 & -1.682162 & 1.097424 \\
\hline 26 & 1 & 0 & -2.763700 & 1.744811 & -0.453222 \\
\hline 27 & 1 & 0 & -1.576631 & 1.976665 & 0.847406 \\
\hline 28 & 1 & 0 & -1.042383 & 1.915431 & -0.842296 \\
\hline
\end{tabular}

! Name Definition Value Derivative Info.

Full file name: amine 38 b ts.log

Method: $\quad$ B $\quad$ LYP / $6-3 \overline{1} G(d)$ opt/freq

Point group: $\quad$ C1

NImag: 1

E (electronic): $\quad-646.104576266$

Zero-point correction=

Thermal correction to Energy=

0.453142 (Hartree/Particle)

Thermal correction to Enthalpy=

0.474959

Thermal correction to Gibbs Free Energy=

0.475903

Sum of electronic and zero-point Energies=

0.404098

$-645.651435$

Sum of electronic and thermal Energies=

$-645.629617$ 
Standard orientation:

\begin{tabular}{|c|c|c|c|c|c|}
\hline \multirow{2}{*}{$\begin{array}{l}\text { Center } \\
\text { Number }\end{array}$} & \multirow{2}{*}{$\begin{array}{l}\text { Atomic } \\
\text { Number }\end{array}$} & \multirow{2}{*}{$\begin{array}{c}\text { Atomic } \\
\text { Type }\end{array}$} & \multicolumn{3}{|c|}{ Coordinates (Angstroms) } \\
\hline & & & $\mathrm{X}$ & Y & Z \\
\hline & & & & & ---- \\
\hline 1 & 7 & 0 & -1.517012 & 0.025958 & 0.324134 \\
\hline 2 & 6 & 0 & 0.963548 & -0.810400 & 0.491998 \\
\hline 3 & 6 & 0 & 1.719050 & 0.236767 & -0.272940 \\
\hline 4 & 6 & 0 & 3.295468 & 0.217374 & -0.199671 \\
\hline 5 & 6 & 0 & 3.777939 & 1.460635 & -0.975552 \\
\hline 6 & 6 & 0 & 0.846340 & -2.183401 & -0.091050 \\
\hline 7 & 6 & 0 & 0.569773 & -0.610147 & 1.870459 \\
\hline 8 & 6 & 0 & 3.791783 & 0.308962 & 1.253619 \\
\hline 9 & 6 & 0 & 3.888897 & -1.035686 & -0.869880 \\
\hline 10 & 6 & 0 & -2.691239 & -0.665488 & -0.252825 \\
\hline 11 & 6 & 0 & -2.400762 & -1.061640 & -1.725019 \\
\hline 12 & 6 & 0 & -2.883422 & -1.962048 & 0.569301 \\
\hline 13 & 6 & 0 & -4.052003 & 0.075818 & -0.216195 \\
\hline 14 & 6 & 0 & -1.410187 & 1.484114 & 0.247411 \\
\hline 15 & 6 & 0 & -1.413533 & 2.105740 & -1.167844 \\
\hline 16 & 6 & 0 & -2.349223 & 2.283528 & 1.189606 \\
\hline 17 & 1 & 0 & 1.448013 & 0.176413 & -1.334302 \\
\hline 18 & 1 & 0 & 1.411258 & 1.224947 & 0.084483 \\
\hline 19 & 1 & 0 & 4.873248 & 1.515221 & -0.974217 \\
\hline 20 & 1 & 0 & 3.445559 & 1.432538 & -2.020412 \\
\hline 21 & 1 & 0 & 3.395730 & 2.384922 & -0.526149 \\
\hline 22 & 1 & 0 & 1.734486 & -2.777527 & 0.179663 \\
\hline 23 & 1 & 0 & -0.017134 & -2.716999 & 0.310757 \\
\hline 24 & 1 & 0 & 0.785809 & -2.172417 & -1.183246 \\
\hline 25 & 1 & 0 & 1.049569 & 0.226834 & 2.381688 \\
\hline 26 & 1 & 0 & -0.570243 & -0.343156 & 1.670991 \\
\hline 27 & 1 & 0 & 0.570667 & -1.517478 & 2.483355 \\
\hline 28 & 1 & 0 & 3.400344 & 1.202668 & 1.754703 \\
\hline 29 & 1 & 0 & 3.491511 & -0.564155 & 1.842749 \\
\hline 30 & 1 & 0 & 4.886810 & 0.367736 & 1.278422 \\
\hline 31 & 1 & 0 & 3.505855 & -1.164730 & -1.889519 \\
\hline 32 & 1 & 0 & 4.980316 & -0.947860 & -0.934563 \\
\hline 33 & 1 & 0 & 3.671712 & -1.949579 & -0.308212 \\
\hline 34 & 1 & 0 & -1.458642 & -1.613636 & -1.796525 \\
\hline 35 & 1 & 0 & -3.201589 & -1.700828 & -2.119459 \\
\hline 36 & 1 & 0 & -2.327002 & -0.181591 & -2.370403 \\
\hline 37 & 1 & 0 & -1.988731 & -2.589393 & 0.538286 \\
\hline 38 & 1 & 0 & -3.086205 & -1.718546 & 1.618129 \\
\hline 39 & 1 & 0 & -3.722863 & -2.554122 & 0.183458 \\
\hline 40 & 1 & 0 & -4.833768 & -0.578792 & -0.622033 \\
\hline 41 & 1 & 0 & -4.337291 & 0.332334 & 0.808907 \\
\hline 42 & 1 & 0 & -4.053986 & 0.990415 & -0.815100 \\
\hline 43 & 1 & 0 & -0.408958 & 1.689892 & 0.655519 \\
\hline 44 & 1 & 0 & -2.400624 & 2.067832 & -1.639487 \\
\hline 45 & 1 & 0 & -1.117633 & 3.161653 & -1.115741 \\
\hline 46 & 1 & 0 & -0.705482 & 1.588714 & -1.824007 \\
\hline 47 & 1 & 0 & -3.367104 & 2.382258 & 0.805051 \\
\hline 48 & 1 & 0 & -2.398736 & 1.802581 & 2.172394 \\
\hline 49 & 1 & 0 & -1.950386 & 3.297374 & 1.327379 \\
\hline
\end{tabular}

\section{S-114}


! Name Definition Value Derivative Info.

Full file name: tritbutylamine_ts.log

Method: $\quad$ B3LYP/6-31G(d) opt/freq

Point group: $\quad \mathrm{C} 1$

NImag: 1

E (electronic): $\quad-528.154396075$

Zero-point correction=

Thermal correction to Energy=

Thermal correction to Enthalpy=

0.368831 (Hartree/Particle)

Thermal correction to Gibbs Free Energy=

Sum of electronic and zero-point Energies=

0.387462

sum of electronic and thermal Energies=

0.326243

$-527.785565$

$-527.767878$

Standard orientation:

$\begin{array}{llcl}\text { Center } & \text { Atomic } & \text { Atomic } & \text { Coordinates (Angstroms) } \\ \text { Number } & \text { Number } & \text { Type } & \text { Y }\end{array}$

\begin{tabular}{|c|c|c|c|c|c|}
\hline 1 & 7 & 0 & 0.367444 & 0.026769 & -0.258260 \\
\hline 2 & 6 & 0 & -2.433434 & 0.046035 & -0.089228 \\
\hline 3 & 6 & 0 & 0.995125 & -1.291925 & 0.007659 \\
\hline 4 & 6 & 0 & 1.129707 & 1.264231 & 0.013784 \\
\hline 5 & 6 & 0 & -2.899433 & 1.307838 & 0.566096 \\
\hline 6 & 6 & 0 & -2.150274 & 0.027038 & -1.524757 \\
\hline 7 & 6 & 0 & -2.795683 & -1.202450 & 0.649213 \\
\hline 8 & 6 & 0 & 0.730197 & -1.743589 & 1.470910 \\
\hline 9 & 6 & 0 & 0.324532 & -2.308196 & -0.949059 \\
\hline 10 & 6 & 0 & 2.518782 & -1.454880 & -0.255658 \\
\hline 11 & 6 & 0 & 1.760656 & 1.383959 & 1.426692 \\
\hline 12 & 6 & 0 & 0.142585 & 2.444463 & -0.114108 \\
\hline 13 & 6 & 0 & 2.226886 & 1.520047 & -1.064538 \\
\hline 14 & 1 & 0 & -4.003028 & 1.274330 & 0.627443 \\
\hline 15 & 1 & 0 & -2.539720 & 1.402584 & 1.596752 \\
\hline 16 & 1 & 0 & -2.630471 & 2.203175 & 0.005714 \\
\hline 17 & 1 & 0 & -1.011513 & 0.079130 & -1.569835 \\
\hline 18 & 1 & 0 & -2.453328 & -0.902899 & -2.018110 \\
\hline 19 & 1 & 0 & -2.555062 & 0.895870 & -2.055647 \\
\hline 20 & 1 & 0 & -3.891302 & -1.337136 & 0.579101 \\
\hline 21 & 1 & 0 & -2.336763 & -2.101819 & 0.239042 \\
\hline 22 & 1 & 0 & -2.561371 & -1.135426 & 1.716860 \\
\hline 23 & 1 & 0 & -0.330561 & -1.656523 & 1.717667 \\
\hline 24 & 1 & 0 & 1.030621 & -2.790171 & 1.611894 \\
\hline 25 & 1 & 0 & 1.291681 & -1.138078 & 2.187000 \\
\hline 26 & 1 & 0 & -0.758525 & -2.333300 & -0.827503 \\
\hline 27 & 1 & 0 & 0.534643 & -2.038934 & -1.990068 \\
\hline 28 & 1 & 0 & 0.705405 & -3.321740 & -0.772720 \\
\hline 29 & 1 & 0 & 2.798355 & -2.498471 & -0.064248 \\
\hline 30 & 1 & 0 & 2.768294 & -1.236644 & -1.298459 \\
\hline 31 & 1 & 0 & 3.144134 & -0.833713 & 0.38889 \\
\hline 32 & 1 & 0 & 2.565731 & 0.666792 & 1.60274 \\
\hline 33 & 1 & 0 & 2.183727 & 2.387554 & 1.56306 \\
\hline
\end{tabular}

\section{S-115}




\begin{tabular}{|c|c|c|c|c|c|}
\hline 34 & 1 & 0 & 0.997413 & 1.236247 & 2.198959 \\
\hline 35 & 1 & 0 & -0.394389 & 2.403782 & -1.067714 \\
\hline 36 & 1 & 0 & -0.583326 & 2.431533 & 0.700670 \\
\hline 37 & 1 & 0 & 0.676238 & 3.401130 & -0.067367 \\
\hline 38 & 1 & 0 & 3.093512 & 0.867128 & -0.964296 \\
\hline 39 & 1 & 0 & 1.803364 & 1.378562 & -2.064793 \\
\hline 40 & 1 & 0 & 2.586252 & 2.554222 & -0.989248 \\
\hline
\end{tabular}

! Name Definition Value Derivative Info.

Full file name: tbutylamine tsh2o.log

Method: B3LYP/6-31G (d) opt/freq

Point group: $\quad$ C1

NImag: $\quad 1$

E (electronic): $\quad-290.137353164$

Zero-point correction=

0.167966 (Hartree/Particle)

Thermal correction to Energy=

0.176653

Thermal correction to Enthalpy=

0.177597

Thermal correction to Gibbs Free Energy=

Sum of electronic and zero-point Energies=

0.135146

$-289.969387$

Sum of electronic and thermal Energies=

$-289.960700$

Standard orientation:

\begin{tabular}{|c|c|c|c|c|c|}
\hline \multirow{2}{*}{$\begin{array}{l}\text { Center } \\
\text { Number }\end{array}$} & \multirow{2}{*}{$\begin{array}{l}\text { Atomic } \\
\text { Number }\end{array}$} & \multirow{2}{*}{$\begin{array}{c}\text { Atomic } \\
\text { Type }\end{array}$} & \multicolumn{3}{|c|}{ Coordinates (Angstroms) } \\
\hline & & & $\mathrm{X}$ & Y & Z \\
\hline & & & & - & - \\
\hline 1 & 7 & 0 & 0.365514 & 1.339246 & 0.084144 \\
\hline 2 & 6 & 0 & -0.648433 & -0.211510 & -0.018255 \\
\hline 3 & 6 & 0 & -1.320963 & -0.167425 & 1.347886 \\
\hline 4 & 6 & 0 & -1.580799 & 0.164582 & -1.161386 \\
\hline 5 & 6 & 0 & 0.263045 & -1.315131 & -0.248903 \\
\hline 6 & 1 & 0 & 0.261644 & 1.897692 & -0.763000 \\
\hline 7 & 1 & 0 & 0.128801 & 1.925927 & 0.883941 \\
\hline 8 & 1 & 0 & -2.056490 & -0.978426 & 1.404660 \\
\hline 9 & 1 & 0 & -0.593428 & -0.329406 & 2.150819 \\
\hline 10 & 1 & 0 & -1.849797 & 0.775974 & 1.534038 \\
\hline 11 & 1 & 0 & -1.039723 & 0.202983 & -2.113299 \\
\hline 12 & 1 & 0 & -2.351876 & -0.609358 & -1.256185 \\
\hline 13 & 1 & 0 & -2.087513 & 1.124974 & -1.001463 \\
\hline 14 & 1 & 0 & 0.166831 & -1.777603 & -1.233682 \\
\hline 15 & 1 & 0 & 1.605475 & -0.761357 & -0.213953 \\
\hline 16 & 1 & 0 & 0.263634 & -2.058784 & 0.552189 \\
\hline 17 & 1 & 0 & 1.381653 & 0.969753 & 0.131560 \\
\hline 18 & 8 & 0 & 2.545987 & -0.033547 & -0.112303 \\
\hline 19 & 1 & 0 & 2.967192 & -0.311809 & 0.717739 \\
\hline
\end{tabular}

\section{S-116}


! Name Definition Value Derivative Info.

Full file name: ditbutylamine tsh2o.log

Method: B3LYP/6-31G(d) opt/freq

Point group: $\quad$ C1

NImag: $\quad 1$

E (electronic): $\quad-447.386249140$

Zero-point correction=

Thermal correction to Energy=

0.282044 (Hartree/Particle)

Thermal correction to Enthalpy=

0.295849

Thermal correction to Gibbs Free Energy=

Sum of electronic and zero-point Energies=

0.296793

sum of electronic and thermal Energies=

0.244051

$-447.104205$

$-447.090400$

Standard orientation:

\begin{tabular}{|c|c|c|c|c|c|}
\hline \multirow{2}{*}{$\begin{array}{l}\text { Center } \\
\text { Number }\end{array}$} & \multirow{2}{*}{$\begin{array}{l}\text { Atomic } \\
\text { Number }\end{array}$} & \multirow{2}{*}{$\begin{array}{c}\text { Atomic } \\
\text { Type }\end{array}$} & \multicolumn{3}{|c|}{ Coordinates (Angstroms) } \\
\hline & & & $\mathrm{X}$ & Y & Z \\
\hline 1 & 7 & 0 & 0.148874 & 0.011393 & -0.657891 \\
\hline 2 & 6 & 0 & -1.587495 & -0.378594 & 0.045553 \\
\hline 3 & 6 & 0 & -1.424180 & -1.756975 & 0.656725 \\
\hline 4 & 6 & 0 & -1.952165 & 0.729570 & 0.905382 \\
\hline 5 & 6 & 0 & -2.318541 & -0.411256 & -1.291828 \\
\hline 6 & 6 & 0 & 1.498908 & -0.229028 & -0.031972 \\
\hline 7 & 6 & 0 & 1.883537 & -1.712052 & -0.134375 \\
\hline 8 & 6 & 0 & 2.513105 & 0.622774 & -0.825506 \\
\hline 9 & 6 & 0 & 1.478263 & 0.245363 & 1.429188 \\
\hline 10 & 1 & 0 & 0.164897 & -0.357968 & -1.609916 \\
\hline 11 & 1 & 0 & -2.425238 & -2.123981 & 0.919365 \\
\hline 12 & 1 & 0 & -0.973757 & -2.481575 & -0.030843 \\
\hline 13 & 1 & 0 & -0.840617 & -1.728754 & 1.580095 \\
\hline 14 & 1 & 0 & -1.086711 & 1.800653 & 0.556327 \\
\hline 15 & 1 & 0 & -2.919583 & 1.168027 & 0.643568 \\
\hline 16 & 1 & 0 & -1.882119 & 0.518807 & 1.975123 \\
\hline 17 & 1 & 0 & -3.366421 & -0.678677 & -1.110673 \\
\hline 18 & 1 & 0 & -2.308430 & 0.573023 & -1.772069 \\
\hline 19 & 1 & 0 & -1.906258 & -1.151855 & -1.988107 \\
\hline 20 & 1 & 0 & 1.233425 & -2.351230 & 0.467343 \\
\hline 21 & 1 & 0 & 1.845174 & -2.062801 & -1.174299 \\
\hline 22 & 1 & 0 & 2.910603 & -1.851714 & 0.220185 \\
\hline 23 & 1 & 0 & 2.240421 & 1.680770 & -0.769684 \\
\hline 24 & 1 & 0 & 3.521586 & 0.496237 & -0.413973 \\
\hline 25 & 1 & 0 & 2.543882 & 0.322910 & -1.881291 \\
\hline 26 & 1 & 0 & 2.484660 & 0.154525 & 1.853464 \\
\hline 27 & 1 & 0 & 1.162268 & 1.290335 & 1.485577 \\
\hline 28 & 1 & 0 & 0.801527 & -0.356286 & 2.042593 \\
\hline 29 & 1 & 0 & 0.020972 & 1.063150 & -0.689529 \\
\hline 30 & 8 & 0 & -0.254967 & 2.517566 & 0.027288 \\
\hline 31 & 1 & 0 & -0.771245 & 3.197308 & -0.435319 \\
\hline
\end{tabular}




$$
\begin{array}{lllll}
1 & -0.000000050 & -0.000000277 & -0.000000530
\end{array}
$$

Full file name: amine_38b_tsh20.log

Method: $\quad$ B $3 L Y P / 6-3 \overline{1} G(d)$ opt/freq

Point group: $\quad \mathrm{C} 1$

NImag: $\quad 1$

E(electronic): $\quad-722.542747679$

Zero-point correction=

Thermal correction to Energy=

Thermal correction to Enthalpy=

0.482396 (Hartree/Particle)

Thermal correction to Gibbs Free Energy=

Sum of electronic and zero-point Energies=
Sum of electronic and thermal Energies=

0.505074

0.506018

0.434318

$-722.060351$

$-722.037673$

Standard orientation:

$\begin{array}{llll}\text { Center } & \text { Atomic } & \text { Atomic } & \text { Coordinates (Angstroms) } \\ \text { Number } & \text { Number } & \text { Type } & \text { X }\end{array}$

$\begin{array}{rrrrrr}1 & 7 & 0 & -1.276898 & 0.083621 & -0.080201 \\ 2 & 6 & 0 & 0.772321 & 0.896131 & 0.059100 \\ 3 & 6 & 0 & 1.548758 & -0.394340 & -0.103264 \\ 4 & 6 & 0 & 3.118953 & -0.358031 & 0.012035 \\ 5 & 6 & 0 & 3.595509 & -1.774386 & -0.381318 \\ 6 & 6 & 0 & 0.734031 & 1.543044 & 1.419269 \\ 7 & 6 & 0 & 0.701885 & 1.855943 & -1.038693 \\ 8 & 6 & 0 & 3.761273 & 0.658950 & -0.947366 \\ 9 & 6 & 0 & 3.595276 & -0.082999 & 1.452505 \\ 10 & 6 & 0 & -2.076061 & -0.452447 & 1.089903 \\ 11 & 6 & 0 & -1.342749 & -1.584371 & 1.832156 \\ 12 & 6 & 0 & -2.338214 & 0.710624 & 2.076641 \\ 13 & 6 & 0 & -3.479166 & -0.950334 & 0.662298 \\ 14 & 6 & 0 & -1.334168 & -0.594298 & -1.423093 \\ 15 & 6 & 0 & -1.102479 & -2.113639 & -1.443770 \\ 16 & 6 & 0 & -2.567220 & -0.206473 & -2.270941 \\ 17 & 1 & 0 & 1.200353 & -1.130424 & 0.627708 \\ 18 & 1 & 0 & 1.329819 & -0.793153 & -1.097941 \\ 19 & 1 & 0 & 4.687159 & -1.846672 & -0.310052 \\ 20 & 1 & 0 & 3.166371 & -2.538071 & 0.278912 \\ 21 & 1 & 0 & 3.310051 & -2.020211 & -1.411060 \\ 22 & 1 & 0 & 1.659474 & 2.117243 & 1.556192 \\ 23 & 1 & 0 & -0.091919 & 2.255709 & 1.470226 \\ 24 & 1 & 0 & -0.448007 & 2.239006 & -1.122926 \\ 25 & 1 & 0.663801 & 0.828239 & 2.241224 \\ 26 & 1 & 0 & 1.073369 & 1.484745 & -1.996115 \\ 27 & 1 & 0 & 1.207240 & 2.794131 & -0.778123 \\ 28 & 1 & 0 & 3.457613 & 0.477819 & -1.985125 \\ 29 & 1 & 0 & 3.491445 & 1.689534 & -0.696972 \\ 30 & 1 & 0 & 3.854419 & 0.579929 & -0.902187 \\ 31 & 1 & 0 & 3.110594 & -0.756424 & 2.170178 \\ 32 & 1 & 0 & 3.677462 & -0.245103 & 1.527270 \\ 33 & 1 & 0 & 02268 & 0.945235 & 1.771176\end{array}$




$\begin{array}{rrrrrr}34 & 1 & 0 & -0.373038 & -1.249795 & 2.212907 \\ 35 & 1 & 0 & -1.940323 & -1.905582 & 2.693528 \\ 36 & 1 & 0 & -1.178484 & -2.459983 & 1.199566 \\ 37 & 1 & 0 & -1.450913 & 0.993730 & 2.643276 \\ 38 & 1 & 0 & -2.709092 & 1.589787 & 1.540590 \\ 39 & 1 & 0 & -3.099023 & 0.402823 & 2.801934 \\ 40 & 1 & 0 & -4.026801 & -1.266376 & 1.557991 \\ 41 & 1 & 0 & -4.048278 & -0.147484 & 0.185287 \\ 42 & 1 & 0 & -3.446434 & -1.804215 & -0.015744 \\ 43 & 1 & 0 & -0.491890 & -0.147507 & -1.958917 \\ 44 & 1 & 0 & -1.953615 & -2.679911 & -1.055496 \\ 45 & 1 & 0 & -0.963682 & -2.425800 & -2.485681 \\ 46 & 1 & 0 & -0.212162 & -2.417138 & -0.887745 \\ 47 & 1 & 0 & -3.472352 & -0.747130 & -1.981579 \\ 48 & 1 & 0 & -2.747608 & 0.869391 & -2.192318 \\ 49 & 1 & 0 & -2.360025 & -0.455330 & -3.319767 \\ 50 & 1 & 0 & -1.634097 & 1.079944 & -0.268116 \\ 51 & 8 & 0 & -1.912127 & 2.487942 & -0.887231 \\ 52 & 1 & 0 & -1.936083 & 3.279920 & -0.325610 \\ ---------------------------------------------------\end{array}$

! Name Definition $\quad$ Value Derivative Info.

Full file name: tritbutylamine_tsh2o.log

Method: B3LYP/6-31G(d) opt/freq

Point group: $\quad$ C1

NImag: $\quad 1$

E (electronic): $\quad-604.588838328$

Zero-point correction=

Thermal correction to Energy=

Thermal correction to Enthalpy=

0.415905

Thermal correction to Gibbs Free Energy=

Sum of electronic and zero-point Energies=

Sum of electronic and thermal Energies=

0.397306 (Hartree/Particle)
0.415905
0.416850
0.355170
$\quad-604.191532$
-604.172933

Standard orientation:

\begin{tabular}{|c|c|c|c|c|c|}
\hline \multirow{2}{*}{$\begin{array}{l}\text { Center } \\
\text { Number }\end{array}$} & \multirow{2}{*}{$\begin{array}{l}\text { Atomic } \\
\text { Number }\end{array}$} & \multirow{2}{*}{$\begin{array}{c}\text { Atomic } \\
\text { Type }\end{array}$} & \multicolumn{3}{|c|}{ Coordinates (Angstroms) } \\
\hline & & & $\mathrm{X}$ & $\mathrm{Y}$ & Z \\
\hline 1 & 7 & 0 & 0.243572 & 0.006747 & 0.120628 \\
\hline 2 & 6 & 0 & -1.990689 & -0.030915 & -0.359662 \\
\hline 3 & 6 & 0 & 0.921351 & 1.369801 & -0.031595 \\
\hline 4 & 6 & 0 & 1.006013 & -1.268077 & -0.239368 \\
\hline 5 & 6 & 0 & -1.968725 & -0.429904 & -1.812785 \\
\hline 6 & 6 & 0 & -2.473734 & -0.945325 & 0.678590 \\
\hline 7 & 6 & 0 & -2.478552 & 1.379677 & -0.131867 \\
\hline 8 & 6 & 0 & 0.734934 & 1.958338 & -1.445972 \\
\hline 9 & 6 & 0 & 0.320284 & 2.325302 & 1.035602 \\
\hline 10 & 6 & 0 & 2.447083 & 1.385722 & 0.249269 \\
\hline 11 & 6 & 0 & 1.520579 & -1.291812 & -1.688938 \\
\hline 12 & 6 & 0 & 0.089312 & -2.494795 & -0.028473 \\
\hline
\end{tabular}

\section{S-119}




$\begin{array}{rrrrrr}13 & 6 & 0 & 2.183455 & -1.527754 & 0.752714 \\ 14 & 1 & 0 & -3.011610 & -0.383478 & -2.165530 \\ 15 & 1 & 0 & -1.386256 & 0.256180 & -2.431056 \\ 16 & 1 & 0 & -1.622018 & -1.447606 & -1.983422 \\ 17 & 1 & 0 & -1.749882 & -0.841406 & 1.607842 \\ 18 & 1 & 0 & -3.427997 & -0.583709 & 1.081434 \\ 19 & 1 & 0 & -2.567153 & -1.985586 & 0.364548 \\ 20 & 1 & 0 & -3.547183 & 1.370511 & -0.396481 \\ 21 & 1 & 0 & -2.406369 & 1.662916 & 0.918872 \\ 22 & 1 & 0 & -2.003373 & 2.131348 & -0.760695 \\ 23 & 1 & 0 & -0.313752 & 2.030299 & -1.736468 \\ 24 & 1 & 0 & 1.150298 & 2.972640 & -1.473682 \\ 25 & 1 & 0 & 1.253862 & 1.370983 & -2.208682 \\ 26 & 1 & 0 & -0.630597 & 2.758571 & 0.732112 \\ 27 & 1 & 0 & 0.174901 & 1.794535 & 1.981975 \\ 28 & 1 & 0 & 1.006799 & 3.162883 & 1.199305 \\ 29 & 1 & 0 & 2.788961 & 2.421542 & 0.146295 \\ 30 & 1 & 0 & 2.677271 & 1.064682 & 1.267826 \\ 31 & 1 & 0 & 3.029403 & 0.790756 & -0.456149 \\ 32 & 1 & 0 & 2.286048 & -0.535941 & -1.879842 \\ 33 & 1 & 0 & 1.974830 & -2.268600 & -1.893320 \\ 34 & 1 & 0 & 0.711119 & -1.144825 & -2.409796 \\ 35 & 1 & 0 & -0.280986 & -2.520720 & 0.998078 \\ 36 & 1 & 0 & -0.760105 & -2.528595 & -0.707520 \\ 37 & 1 & 0 & 0.675947 & -3.401991 & -0.205921 \\ 38 & 1 & 0 & 3.087285 & -0.965972 & 0.527089 \\ 39 & 1 & 0 & 1.867159 & -1.294889 & 1.773678 \\ 40 & 1 & 0 & 2.453006 & -2.589075 & 0.715385 \\ 41 & 1 & 0 & 0.000392 & -0.130244 & 1.177130 \\ 42 & 8 & 0 & -0.591518 & -0.336859 & 2.555475 \\ 43 & 1 & 0 & -0.270730 & -1.099103 & 3.063711 \\ -----------------------------190\end{array}$

! Name Definition Value Derivative Info.

Full file name: etnme2h nh3 E2.log

Method: B3LYP/6-31G(d) opt/freq

Point group: CS

NImag: $\quad 1$

E (electronic): $\quad-270.672761161$

Zero-point correction=

Thermal correction to Energy=

Thermal correction to Enthalpy=

Thermal correction to Gibbs Free Energy=

Sum of electronic and zero-point Energies=

Sum of electronic and thermal Energies=

0.196365 (Hartree/Particle)
0.207032
0.207976
0.158776
$\quad-270.476396$
$\quad-270.465729$

Standard orientation: 


\begin{tabular}{|c|c|c|c|c|c|}
\hline \multirow{2}{*}{$\begin{array}{l}\text { Center } \\
\text { Number }\end{array}$} & \multirow{2}{*}{$\begin{array}{l}\text { Atomic } \\
\text { Number }\end{array}$} & \multirow{2}{*}{$\begin{array}{l}\text { Atomic } \\
\text { Type }\end{array}$} & \multicolumn{3}{|c|}{ Coordinates (Angstroms) } \\
\hline & & & $\mathrm{X}$ & $\mathrm{Y}$ & Z \\
\hline 1 & 7 & 0 & 1.808064 & -0.447648 & 0.000000 \\
\hline 2 & 6 & 0 & -0.120307 & 0.777892 & -0.000000 \\
\hline 3 & 6 & 0 & -1.243864 & -0.013001 & -0.000000 \\
\hline 4 & 6 & 0 & 1.808064 & -1.252888 & 1.223321 \\
\hline 5 & 6 & 0 & 1.808064 & -1.252888 & -1.223321 \\
\hline 6 & 1 & 0 & 2.606459 & 0.185584 & 0.000000 \\
\hline 7 & 1 & 0 & 0.235233 & 1.234988 & 0.918349 \\
\hline 8 & 1 & 0 & 0.235233 & 1.234988 & -0.918349 \\
\hline 9 & 1 & 0 & -2.435366 & 1.216588 & -0.000000 \\
\hline 10 & 1 & 0 & -1.558135 & -0.503050 & 0.921964 \\
\hline 11 & 1 & 0 & -1.558135 & -0.503050 & -0.921964 \\
\hline 12 & 1 & 0 & 1.905633 & -0.599621 & 2.095247 \\
\hline 13 & 1 & 0 & 0.855721 & -1.790232 & 1.294228 \\
\hline 14 & 1 & 0 & 2.621266 & -1.992168 & 1.243851 \\
\hline 15 & 1 & 0 & 1.905633 & -0.599621 & -2.095247 \\
\hline 16 & 1 & 0 & 0.855721 & -1.790232 & -1.294228 \\
\hline 17 & 1 & 0 & 2.621266 & -1.992168 & -1.243851 \\
\hline 18 & 7 & 0 & -3.360179 & 1.886372 & -0.000000 \\
\hline 19 & 1 & 0 & -4.206589 & 1.308457 & -0.000000 \\
\hline 20 & 1 & 0 & -3.365441 & 2.481891 & 0.833988 \\
\hline 21 & 1 & 0 & -3.365441 & 2.481891 & -0.833988 \\
\hline
\end{tabular}
1
7
$-0.000000098$
0.000000092
0.000000000

Full file name: tbunme2h_nh3_E2.log

Method: B3LYP/6-3iG $(\bar{d})$ opt/freq

Point group: CS

NImag: 1

E(electronic): $\quad-349.312387203$

Zero-point correction=

Thermal correction to Energy=

Thermal correction to Enthalpy=

Thermal correction to Gibbs Free Energy=

Sum of electronic and zero-point Energies=

Sum of electronic and thermal Energies=

0.249169 (Hartree/Particle)
0.263353
0.264297
0.207409
$\quad-349.063218$
$\quad-349.049035$

Standard orientation:

$\begin{array}{llll}\text { Center } & \text { Atomic } & \text { Atomic } & \text { Coordinates (Angstroms) } \\ \text { Number } & \text { Number } & \text { Type } & \text { X }\end{array}$

$\begin{array}{rrrrrr}1 & 7 & 0 & -1.223822 & 1.659989 & 0.000000 \\ 2 & 6 & 0 & -0.107431 & -0.729196 & -0.000000 \\ 3 & 6 & 0 & 1.230917 & -0.366183 & -0.000000 \\ 4 & 6 & 0 & -0.796015 & -1.119203 & 1.276747 \\ 5 & 6 & 0 & -0.796015 & -1.119203 & -1.276747 \\ 6 & 6 & 0 & -0.796015 & 2.353626 & 1.217141 \\ 7 & 6 & 0 & -0.796015 & 2.353626 & -1.217141\end{array}$




$\begin{array}{rrrrrr}8 & 1 & 0 & -2.240958 & 1.590315 & 0.000000 \\ 9 & 1 & 0 & 1.926355 & -1.667084 & -0.000000 \\ 10 & 1 & 0 & 1.653887 & 0.041631 & -0.919702 \\ 11 & 1 & 0 & 1.653887 & 0.041631 & 0.919702 \\ 12 & 1 & 0 & -1.845525 & -0.815368 & 1.266130 \\ 13 & 1 & 0 & -0.792508 & -2.215383 & 1.372271 \\ 14 & 1 & 0 & -0.308675 & -0.697335 & 2.158869 \\ 15 & 1 & 0 & -1.845525 & -0.815368 & -1.266130 \\ 16 & 1 & 0 & -0.792508 & -2.215383 & -1.372271 \\ 17 & 1 & 0 & -0.308675 & -0.697335 & -2.158869 \\ 18 & 1 & 0 & 0.297334 & 2.314834 & 1.287773 \\ 19 & 1 & 0 & -1.095661 & 3.412820 & 1.239591 \\ 20 & 1 & 0 & -1.217328 & 1.856678 & 2.095960 \\ 21 & 1 & 0 & 0.297334 & 2.314834 & -1.287773 \\ 22 & 1 & 0 & -1.095661 & 3.412820 & -1.239591 \\ 23 & 1 & 0 & -1.217328 & 1.856678 & -2.095960 \\ 24 & 7 & 0 & 2.717735 & -2.674766 & -0.000000 \\ 25 & 1 & 0 & 2.575393 & -3.254769 & -0.830330 \\ 26 & 1 & 0 & 3.686805 & -2.346811 & -0.000000 \\ 27 & 1 & 0 & 2.575393 & -3.254769 & 0.830330 \\ ---------------------------------------------------------\end{array}$

Water

Full file name: water.log

Method: B3LYP/6-31G(d) opt/freq

Point group: $\quad \mathrm{C} 2 \mathrm{~V}$

NImag: $\quad 0$

E (electronic): $\quad-76.4089539793$

Thermal correction to Energy=

0.021163 (Hartree/Particle)

Thermal correction to Enthalpy=

0.023997

0.024941

Thermal correction to Gibbs Free Energy=

Sum of electronic and zero-point Energies=

0.003495

Sum of electronic and thermal Energies=

$-76.387791$

$-76.384957$

Standard orientation:

\begin{tabular}{|c|c|c|c|c|c|}
\hline \multirow{2}{*}{$\begin{array}{l}\text { Center } \\
\text { Number }\end{array}$} & \multirow{2}{*}{$\begin{array}{l}\text { Atomic } \\
\text { Number }\end{array}$} & \multirow{2}{*}{$\begin{array}{c}\text { Atomic } \\
\text { Type }\end{array}$} & \multicolumn{3}{|c|}{ Coordinates (Angstroms) } \\
\hline & & & $\mathrm{X}$ & $\mathrm{Y}$ & $\mathrm{Z}$ \\
\hline 1 & 8 & 0 & 0.000000 & 0.000000 & 0.119746 \\
\hline 2 & 1 & 0 & -0.000000 & 0.761555 & -0.478986 \\
\hline 3 & 1 & 0 & -0.000000 & -0.761555 & -0.478986 \\
\hline
\end{tabular}

Ammonia 
Full file name: ammonia.log

Method: B3LYP/6-31G(d) opt/freq

Point group: $\quad$ C $3 \mathrm{~V}$

NImag: $\quad 0$

E (electronic) : $\quad-56.5479477986$

Zero-point correction=

Thermal correction to Energy=

0.034533 (Hartree/Particle)

Thermal correction to Enthalpy=

0.037391

Thermal correction to Gibbs Free Energy=

Sum of electronic and zero-point Energies=

0.038336

Sum of electronic and thermal Energies=

0.016491

$-56.513415$

$-56.510556$

Standard orientation:

\begin{tabular}{|c|c|c|c|c|c|}
\hline \multirow{2}{*}{$\begin{array}{l}\text { Center } \\
\text { Number }\end{array}$} & \multirow{2}{*}{$\begin{array}{l}\text { Atomic } \\
\text { Number }\end{array}$} & \multirow{2}{*}{$\begin{array}{c}\text { Atomic } \\
\text { Type }\end{array}$} & \multicolumn{3}{|c|}{ Coordinates (Angstroms) } \\
\hline & & & $\mathrm{X}$ & Y & Z \\
\hline 1 & 7 & 0 & 0.000000 & 0.000000 & 0.119354 \\
\hline 2 & 1 & 0 & 0.000000 & 0.938600 & -0.278494 \\
\hline 3 & 1 & 0 & -0.812851 & -0.469300 & -0.278494 \\
\hline 4 & 1 & 0 & 0.812851 & -0.469300 & -0.278494 \\
\hline
\end{tabular}

